Pontifícia Universidade $C_{\text {atólica }}$

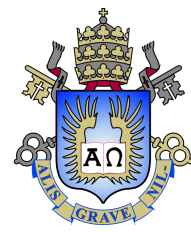

Diogo Cardoso Lima

\title{
Métodos Baseados em Medicões Fasoriais \\ Sincronizadas para o Monitoramento da \\ Estabilidade de Tensão
}

Dissertação de Mestrado

Dissertação apresentada ao Programa de Pós-Graduação em Engenharia Elétrica da PUC-Rio como requisito parcial para obtenção do grau de Mestre em Engenharia Elétrica.

Orientador : $\quad$ Prof. Ricardo Bernardo Prada Co-orientador: Prof. Marcos Vinícius Pimentel Teixeira 
Diogo Cardoso Lima

\title{
Métodos Baseados em Medições Fasoriais \\ Sincronizadas para o Monitoramento da \\ Estabilidade de Tensão
}

Dissertação apresentada como requisito parcial para obtenção do grau de Mestre pelo Programa de Pós-Graduação em Engenharia Elétrica da PUC-Rio. Aprovada pela Comissão Examinadora abaixo assinada.

\author{
Prof. Ricardo Bernardo Prada \\ Orientador \\ Departamento de Engenharia Elétrica - PUC-Rio
}

Prof. Marcos Vinícius Pimentel Teixeira

Co-orientador Instituto Militar de Engenharia - IME

Prof. Julio Cesar Stacchini de Souza Universidade Federal Fluminense - UFF

Profa. Tatiana Mariano Lessa de Assis Universidade Federal do Rio de Janeiro - UFRJ 
Todos os direitos reservados. É proibida a reprodução total ou parcial do trabalho sem autorização da universidade, do autor e do orientador.

\section{Diogo Cardoso Lima}

Graduou-se em 2016 no curso de Engenharia Elétrica com ênfase em Sistemas de Potência pela Universidade do Estado do Rio de Janeiro (UERJ).

Ficha Catalográfica

Lima, Diogo Cardoso

Métodos baseados em medições fasoriais sincronizadas para o monitoramento da estabilidade de tensão / Diogo Cardoso Lima; orientador: Ricardo Bernardo Prada; coorientador: Marcos Vinícius Pimentel Teixeira. - 2019.

126 f.: il. color. ; $30 \mathrm{~cm}$

Dissertação (mestrado) - Pontifícia Universidade Católica do Rio de Janeiro, Departamento de Engenharia Elétrica, 2019

Inclui bibliografia

1. Engenharia Elétrica - Teses. 2. Estabilidade de tensão;. 3. Medições fasoriais sincronizadas;. 4. Equivalente de Thévenin;. 5. Circuito Acoplado de Portas Simples Aprimorado;. 6. Máximo carregamento;. I. Prada, Ricardo Bernardo. II. Teixeira, Marcos Vinícius Pimentel. III. Pontifícia Universidade Católica do Rio de Janeiro. Departamento de Engenharia Elétrica. IV. Título. 
Aos meus pais, Adilson e Ana, pelo amor, apoio e confiança. 


\section{Agradecimentos}

Agradeço a Deus por dar-me forças, e sem o qual nada teria sentido.

Aos meus pais, Adilson Ferreira Lima e Ana Cristina Cardoso Lima, por seu amor incondicional e incansável incentivo que me sustentaram durante esta empreitada.

Às minhas irmãs Daienne Cardoso Lima e Dyana Cardoso Lima, que estão sempre ao meu lado e que partilham deste momento com alegria.

À Gabriele Vascouto por seu companheirismo e compreensão nos momentos difíceis.

Ao meu orientador Ricardo Bernardo Prada pela confiança, e por mostrar-se solícito e disposto a dividir o seu conhecimento, lançando um olhar atencioso sobre o presente trabalho.

Ao meu co-orientador Marcos Vinícius Pimentel Teixeira pelas valiosas contribuições sobre o tema.

Aos professores do Departamento de Engenharia Elétrica da PUC Rio pelo constante aprendizado.

Aos laços de amizade construídos durante este período de pós-graduação. Em particular, registro meus agradecimento a Igor de Oliveira Barreto, Marília Zacarias, Lívia Cristina da Silva Santos, Patrícia Sales Mansano e Túlio Anselmo dos Santos Valentim.

Agradeço à Pontifícia Universidade Católica do Rio de Janeiro pelos auxílios concedidos e pela infraestrutura que viabilizaram este trabalho.

O presente trabalho foi realizado com o apoio da Coordenação de Aperfeiçoamento de Pessoal de Nível Superior - Brasil (CAPES) - Código de Financiamento 001. 


\section{Resumo}

Lima, Diogo Cardoso; Prada, Ricardo Bernardo; Teixeira, Marcos Vinícius Pimentel. Métodos Baseados em Medições Fasoriais Sincronizadas para o Monitoramento da Estabilidade de Tensão. Rio de Janeiro, 2019. 126p. Dissertação de Mestrado Departamento de Engenharia Elétrica, Pontifícia Universidade Católica do Rio de Janeiro.

O fenômeno associado à estabilidade de tensão se caracteriza pela existência de valores máximos de potência ativa e reativa que podem ser transferidas a uma barra de carga, ou injetada na rede através dos geradores e compensadores síncronos. Sua relevância para o setor elétrico é constantemente reforçada pelo registro de ocorrências de colapsos de tensão em diversos países do mundo e, embora a instabilidade de tensão manifeste-se localmente, suas consequências se refletem na ampla deterioração do sistema. Impulsionado pelo crescente interesse a respeito dos métodos baseados em medições fasoriais sincronizadas para a detecção do máximo carregamento, este trabalho apresenta os principais modelos encontrados na literatura baseados em medições locais e no vasto monitoramento do sistema, com maior ênfase aos métodos de Corsi-Taranto e Circuito Acoplado de Porta-Simples Aprimorado (CAPS Aprimorado). Algumas contribuições são sugeridas ao modelo CAPS Aprimorado tendo em vista sua aplicação ao conjunto de barras de transferência e de geração. Explora-se a utilização dos equivalentes multi-portas como índice de avaliação das condições de estabilidade de tensão, comparando a identificação da barra crítica aos métodos da matriz $\boldsymbol{D}^{\prime}$ e dos autovalores e autovetores. São evidenciadas as principais limitações dos modelos mediante a um conjunto de análises estáticas e dinâmicas, considerando diferentes cenários de carregamento, a sensibilidade dos modelos ao ruído e a resposta dos métodos em face da atuação dos limitadores de sobre-excitação.

\section{Palavras-chave}

Estabilidade de tensão; Medições fasoriais sincronizadas; Equivalente de Thévenin; Circuito Acoplado de Portas Simples Aprimorado; Máximo carregamento; 


\section{Abstract}

Lima, Diogo Cardoso; Prada, Ricardo Bernardo (Advisor); Teixeira, Marcos Vinícius Pimentel (Co-Advisor). Methods Based on Synchronized Phasor Measurements for Voltage Stability Monitoring.. Rio de Janeiro, 2019. 126p. Dissertação de mestrado

- Departamento de Engenharia Elétrica , Pontifícia Universidade Católica do Rio de Janeiro.

The voltage stability phenomenon is characterized by the existence of a maximum active and reactive power that can be transferred to a load bus or injected into the network through the generators and synchronous compensators. Its relevance to the electric power sector is constantly reinforced by the record of occurrences of voltage collapses in several countries around the world. Although the voltage instability manifests itself locally, its consequences is reflected in the wide deterioration of the system. Driven by growing interest in methods based on synchronized phasor measurements for maximum loadability detection, this work presents the main models found in the literature based on local measurements and wide area monitoring, emphasizing the Corsi-Taranto method and Improved Coupled Single-Port Circuit (Improved CAPS). Some contributions are suggested to the Improved CAPS model considering its application to the set of transit and controlled buses. The use of the multi-port equivalents is explored as the evaluation index of voltage stability conditions, comparing the identification of the critical bus to the $\boldsymbol{D}^{\prime}$ matrix method and eigenvalues and eigenvalues method. The main limitations of the models are demonstrated through a static and dynamic studies, as well different loading scenarios, the sensitivity of the models to the noise and the response of the methods when the over-excitation limiters are considered.

\section{Keywords}

Voltage stability; Synchronized phasor measurement; Thévenin equivalent; Improved Coupled Single Port Circuit; Maximum loadability; 


\section{Sumário}

1 Introdução $\quad 17$

$\begin{array}{lll}1.1 & \text { Unidade de Medição Fasorial } & 18\end{array}$

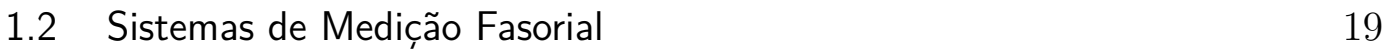

1.3 Objetivo e Levantamento Bibliográfico 20

1.4 Estrutura do Trabalho 20

2 Considerações Gerais sobre Estabilidade $\quad 22$

2.1 Caracterização da Estabilidade de Tensão 24

2.2 Curvas $P_{L}, Q_{L}$ e $\phi$ Constantes 26

2.3 Avaliação da Máxima Transferência de Potência 28

2.4 Demonstração da Existência de $P_{L}^{\text {máx,máx }} 32$

2.5 Conclusões 33

3 Avaliação das Barras de Geração $\quad 34$

3.1 Caracterização da Estabilidade de Tensão a partir da Barra de Geração 34

3.2 Tensão Crítica na Barra Terminal 35

3.3 Exemplos Numéricos 37

3.4 Conclusões 42

4 Avaliação das Condições de Estabilidade de Tensão 43

4.1 Método da Matriz $\boldsymbol{D}^{\prime} \quad 43$

4.1.1 Módulo do Determinante de $\boldsymbol{D}^{\prime} \quad 45$

$\begin{array}{ll}\text { 4.1.2 Sinal de } \operatorname{det}\left(\boldsymbol{D}^{\prime}\right) & 47\end{array}$

4.1.3 Interpretação dos Índices 49

4.1.4 Avaliação de Barras de Tensão Controlada 50

4.2 Análise por Autovalores e Autovetores 52

5 Métodos de Detecção da Instabilidade de Tensão $\quad 55$

5.1 Método VIP 56

5.2 Método de Šmon, Verbič e Gubina (ŠVG) 57

5.3 Método de Corsi-Taranto 59

5.4 Circuito Acoplado de Porta Simples (CAPS) 63

5.5 Circuito Acoplado de Porta Simples Aprimorado (CAPS Aprimorado) 67

5.5.1 Avaliação de Barras de Transferência 70

5.5.2 Avaliação de Barras de Tensão Controlada : Uma Contribuição a partir do Modelo CAPS Aprimorado $\quad 71$

$\begin{array}{lll}6 & \text { Resultados } & 73\end{array}$

6.1 Identificação da Barra de Carga Crítica $\quad 73$

6.2 Avaliação das Barras de Carga $\quad 78$

6.2.1 Considerações sobre o Método CAPS 85

6.2.2 Considerações sobre os Métodos VIP, ŠVG e Corsi-Taranto 89

$\begin{array}{lll}6.3 & \text { Sensibilidade ao Ruído } & 91\end{array}$

6.4 Avaliação das Barras de Transferência 93 
6.5 Avaliação das Barras de Tensão Controlada 98

6.5.1 Sistema Teste Radial de 3 Barras $\quad 99$

6.5.1.1 Caso 1 - Carga Pesada na Extremidade do Sistema Radial 99

6.5.1.2 Caso 2 - Carga Pesada na Barra Intermediária do Sistema Radial 101

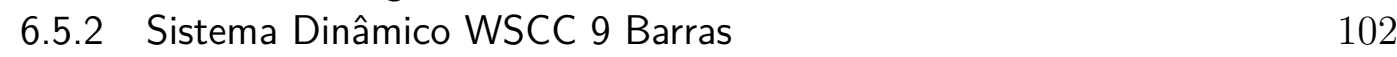

$\begin{array}{lll}\text { 6.5.2.1 Caso } 1 \text { - Cenário sem OXL } & 102\end{array}$

6.5.2.2 Caso 2 - Cenário com OXL 106

$\begin{array}{lll}7 & \text { Conclusões } & 109\end{array}$

$\begin{array}{lll}7.1 & \text { Sugestões para Trabalhos Futuros } & 111\end{array}$

$\begin{array}{ll}\text { Referências bibliográficas } & 113\end{array}$

A Cálculo da Sensibilidade Utilizada no Método CAPS Aprimorado $\quad 117$

B Dados de Linha e Geração $\quad 120$

B.1 Sistema IEEE 14 Barras 120

$\begin{array}{ll}\text { B.2 Sistema WSCC } 9 \text { Barras } & 121\end{array}$

B.3 Sistema IEEE 30 Barras 122

C Dados dos Controles - Sistema WSCC 9 Barras $\quad 124$ 


\section{Lista de figuras}

Figura 1.1 Estrutura simplificada de uma unidade de medição fasorial $[3]$

Figura 1.2 Representação genérica do sistema de medição fasorial [3] 19

Figura 2.1 Classificação de estabilidade de sistemas de potência

Figura 2.2 Potência ativa e reativa drenada pela carga no sistema de duas barras

Figura 2.3 Curva $\theta \times P$ para diferentes valores de tensão na barra de carga

Figura 2.4 Sistema ilustrativo

Figura 2.5 Gráfico representando o lugar geométrico das soluções de tensão para $P_{L}$ e $Q_{L}$ constantes

Figura 2.6 Avaliação da existência de soluções de tensão para diferentes patamares de carga $\operatorname{com} \phi=45^{\circ}$

Figura 2.7 Curva $\left|\dot{S}_{L}\right| \times\left|\dot{V}_{L}\right| \operatorname{com} \phi=45^{\circ}$

Figura 2.8 Curva $\left|\dot{S}_{L}\right| \times\left|\dot{V}_{L}\right|$ para diferentes fatores de potência

Figura 2.9 Lugar geométrico das soluções de tensão para diferentes patamares de carga constante

Figura 3.1 Potência ativa e reativa injetada pela barra terminal no sistema de duas barras

Figura 3.2 Curva $\left|\dot{S}_{G}\right| \times|\dot{V}|$ da barra de geração, com $\phi_{G}=185^{\circ} . \quad 37$

Figura 3.3 Gráfico representando o lugar geométrico das soluções de tensão para $P_{G}$ e $Q_{G}$ constantes

Figura 3.4 Caso 1 - Máxima carga limitada pelo fluxo de potência máximo que chega à barra de carga

Figura 3.5 Caso 2 - Máxima carga limitada simultaneamente pelo fluxo de potência máximo injetado pela barra terminal do gerador, e pelo fluxo que chega à barra de carga

Figura 3.6 Caso 3 - Máxima carga limitada pelo fluxo de potência máximo injetado pela barra terminal do gerador

Figura 4.1 Sistema 2 barras

Figura 4.2 Localização do vetor gradiente $\nabla \vec{P}_{i}$ e $\nabla \vec{Q}_{i}$ no plano $\theta \times|\dot{V}|$

Figura 4.3 Sinal da margem $M$ associado à curva $\phi$ constante em diferentes pontos de operação no plano $|\dot{S}| \times \mid \dot{V}$

Figura 4.4 Variação no tempo da tensão na barra de tensão controlada 51

Figura 4.5 Localização dos pontos de operação na curva $\phi$ constante através do plano $|\dot{S}| \times|\dot{V}|$

Figura 5.1 Barra em análise $j$ do sistema, a rede de Thévenin correspondente $N$, e sua rede adjunta $\hat{N}$

Figura 5.2 Diagrama fasorial do equivalente de Thévenin 60

Figura 5.3 Modelo da rede multi-portas 
Figura 5.4 Equivalente de porta simples para barra $j \quad 65$

Figura 5.5 Construção dos $n$ circuitos de porta simples 66

$\begin{array}{lll}\text { Figura 5.6 Algoritmo do método CAPS } & 67\end{array}$

$\begin{array}{lll}\text { Figura 5.7 Avaliação das barras de transferência } & 70\end{array}$

Figura 6.1 Sistema IEEE 14 barras

Figura 6.2 Identificação da barra de carga crítica no sistema IEEE 14 barras $\quad 76$

$\begin{array}{lll}\text { Figura 6.3 Sistema WSCC } 9 \text { barras } & 78\end{array}$

Figura 6.4 Gráfico de impedância em [pu] - caso $1 \quad 80$

Figura 6.5 $R_{t h}$ e $X_{t h}$ em [pu] - caso 1

Figura 6.6 Gráfico de $\left|\dot{E}_{t h}\right|[\mathrm{pu}]$ - caso $1 \quad 82$

Figura 6.7 Margem de carregamento estimada [\%] - caso 1 82

Figura 6.8 Margem de carregamento estimada [\%] - caso $2 \quad 84$

Figura 6.9 Comportamento de $\left|\bar{Z}_{c j}\right|[\mathrm{pu}]$ para crescimento de carga proporcional no sistema IEEE 14 barras $\quad 86$

Figura 6.10 Comportamento de $\left|\bar{Z}_{c j}\right|[\mathrm{pu}]$ para crescimento de carga desproporcional no sistema IEEE 14 barras 86

Figura 6.11 Sistema radial 4 barras $\quad 87$

Figura 6.12 Margem individual de $L_{1}$ e $L_{2}$ estimadas pelo método CAPS

Figura 6.13 Gráfico de impedância em [pu] para o sistema 4 barras no caso base

Figura 6.14 Influência de $|\Delta \dot{I}|^{\text {mín }}$ pelo método ŠVG através do gráfico de impedância em $[\mathrm{pu}]$

Figura 6.15 Tensão real $\left|\dot{V}_{L}\right|^{\text {real }}$ e tensão medida $\left|\dot{V}_{L}\right|^{\text {medida }}$ na barra 591

Figura 6.16 Resposta do método de Corsi-Taranto à presença de ruído 92

Figura 6.17 Resposta do método CAPS Aprimorado à presença de ruído $\quad 92$

Figura 6.18 Resposta do fator de sensibilidade $\gamma$ à presença de ruído 93

Figura 6.19 Sistema IEEE 30 barras 94

Figura 6.20 Distribuição dos fluxos em [MW] e [Mvar] nos elementos conectados à barra 27

Figura 6.21 Rastreamento da margem de carregamento estimada pelo método CAPS Aprimorado e do ângulo $\beta$ obtido pelo método da matriz $\boldsymbol{D}^{\prime}$ para a barra 27 do sistema teste IEEE 30 barras

Figura 6.22 Sistema teste 3 barras - caso 1

Figura 6.23 Gráfico de impedância em [pu] e margem de carregamento estimada em [\%] para a barra 3 - caso $1 \quad 100$

Figura 6.24 Margem estimada em [\%] para o gerador 1 - caso 11100

Figura 6.25 Sistema teste 3 barras - caso $2 \quad 101$

Figura 6.26 Gráfico de impedância em [pu] e margem de carregamento estimada em [\%] para a barra 3 - caso $2 \quad 102$

Figura 6.27 Margem estimada em [\%] para o gerador 1 - caso 2102

Figura 6.28 Modelo dinâmico do sistema WSCC 9 barras 103

Figura 6.29 Gráfico do módulo da tensão em [pu] - caso 1

Figura 6.30 Potência ativa gerada em [pu] pelos geradores 2 e 3 caso 1 
Figura 6.31 Gráfico de impedância em [pu] e margem de carregamento estimada em [\%] para a barra 5 - caso 1

Figura 6.32 Margem estimada em [\%] para os geradores 2 e 3 - caso 1105

Figura 6.33 Gráfico da módulo da tensão em [pu] - caso $2 \quad 106$

Figura 6.34 Corrente de campo em [pu] dos geradores - caso $2 \quad 107$

Figura 6.35 Gráfico de impedância em [pu] e margem de carregamento estimada em [\%] para a barra 5 - caso $2 \quad 107$

Figura 6.36 Margem estimada em [\%] para os geradores - Sistema $\begin{array}{ll}\text { WSCC } 9 \text { barras - caso } 2 & 108\end{array}$

Figura A.1 Equivalente "visto" pela barra $j$ em análise 117

$\begin{array}{lll}\text { Figura A.2 Diagrama fasorial } & 117\end{array}$

Figura C.1 Regulador de velocidade do tipo II 124

Figura C.2 Regulador automático de tensão do tipo II 125

Figura C.3 Limitador de sobre-excitação 126 


\section{Lista de tabelas}

Tabela 3.1 $\begin{array}{ll}\text { Pontos de operação do caso } 1 & 39\end{array}$

Tabela 3.2 Pontos de operação do caso $2 \quad 40$

Tabela 3.3 Pontos de operação do caso $3 \quad 41$

Tabela 5.1 Características avaliadas por [17] 56

Tabela 6.1 Dados de barra - Sistema IEEE 14 barras 74

Tabela 6.2 Método da Matriz $\boldsymbol{D}^{\prime}$ - Sistema IEEE 14 barras $\quad 75$

Tabela 6.3 Identificação da barra crítica $\quad 77$

Tabela 6.4 Comparação entre as margens estimadas no caso base $\quad 77$

Tabela 6.5 Dados de barra - Sistema WSCC 9 barras 78

Tabela 6.6 Método da Matriz $\boldsymbol{D}^{\prime}$ - Sistema WSCC 9 barras 79

Tabela 6.7 Variações percentuais de carga no sistema WSCC 9 barras 79

Tabela 6.8 Erro de estimação para o caso 1 - Sistema WSCC 9 barras 81

Tabela 6.9 Erro médio quadrático associado à margem de carregamento estimada - caso 1

Tabela 6.10 Erro de estimação para o caso 2 - Sistema WSCC 9 barras 84

Tabela 6.11 Erro médio quadrático associado à margem estimada caso 2

Tabela 6.12 Variações percentuais de carga no sistema IEEE 14 barras 85

Tabela 6.13 Dados de barra - Sistema 4 barras - caso base 87

Tabela 6.14 Erro de estimação para o caso base - Sistema 4 barras $\quad 89$

Tabela 6.15 Dados de barra - Sistema IEEE 30 barras 95

Tabela 6.16 Método da Matriz $\boldsymbol{D}^{\prime}$ - Sistema IEEE 30 barras 96

Tabela B.1 Dados de geração do sistema IEEE 14 barras $\quad 120$

Tabela B.2 Dados de linha do sistema IEEE 14 barras 120

Tabela B.3 Dados de geração do sistema WSCC 9 barras $\quad 121$

Tabela B.4 Dados de linha do sistema WSCC 9 barras 121

Tabela B.5 Dados de geração do sistema IEEE 30 barras 122

Tabela B.6 Dados de linha do sistema IEEE 30 barras 122

Tabela C.1 Dados do regulador de velocidade 124

Tabela C.2 Dados do regulador automático de tensão 125

Tabela C.3 Dados do limitador de sobre-excitação dos geradores 1 e 3126

Tabela C.4 Dados do limitador de sobre-excitação do gerador $2 \quad 126$ 


\section{Lista de Abreviaturas e Siglas}

SMFS Sistema de Medição Fasorial Sincronizada

TDF

Transformada Discreta de Fourier

AM

Amplitude-Modulated

GOES

Geostationary Operational Environmental Satellite

GPS

Global Positioning System

PMU

Phasor Measurement Units

PPS

Pulso Por Segundo

CAPS Aprimorado Circuito Acoplado de Portas Simples Aprimorado

LTC

Load Tap Changer

HVDC

High Voltage Direct Current

LEEA

Limite de Estabilidade Estática Angular

$\mathrm{pu}$

por unidade

LET

Limite de Estabilidade de Tensão

VIP

Voltage Instability Predictor

AEB

American Eletric Power

ŠVG

Šmon, Verbič e Gubina

ISI

Impedance Stability Index

EAT

Extra Alta Tensão

CAPS

Circuito Acoplado de Portas Simples

SCADA

Supervisory Control And Data Aquisition

CAPS Modificado

Circuito Acoplado de Portas Simples Modificado

RPRF

Reactive Power Response Factor

NL

Negative Load Model

MTP

Máxima Transferência de Potência

MATLAB

Matrix Laboratory

FPC

Fluxo de Potência Continuado

ANAREDE

Análise de Redes Elétricas

WSCC

Western System Coordinating Council

EQM

Erro Quadrático Médio

SNR

Signal-to-Noise Ratio

$\mathrm{dB}$

Decibéis

AVR

Automatic Voltage Regulator

OXL

Overexcitation Limiter 
TG

PSAT

$\bar{Z}_{t}$

$\alpha_{t}$

$\bar{Z}_{L}$

$\phi$

$\phi_{G}$

$\Delta \underline{P}$

$\Delta Q$

$\Delta \underline{\mid \dot{V}} \mid$

$\Delta \underline{\theta}$

$J$

$D^{\prime}$

$\left|\dot{S}_{i}\right|^{2}$

$\left|\dot{S}_{i o}^{2}\right|$

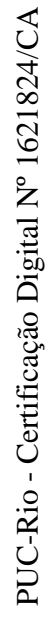

$\Delta \operatorname{det}\left|\dot{V}_{i}\right|$ $S_{m}^{2}$

$\operatorname{det}\left(\boldsymbol{D}^{\prime}\right)\left|\dot{V}_{i}\right|$

$\nabla \vec{P}_{i}$

$\nabla \vec{Q}_{i}$

$\beta$

$M_{i}$

$\boldsymbol{J}_{R}^{Q}$

$\Lambda$

$\lambda_{i}$

$\Phi_{i k}$

$\dot{E}_{t h}$

$\bar{Z}_{t h}$

$R_{t h}$

$X_{t h}$

$|\Delta \dot{I}|^{\text {mín }}$

$\epsilon_{E}$

$k$

$\bar{Z}_{e q_{j}}$
Turbine Governor

Power System Analysis Toolbox

Impedância da linha de transmissão

Ângulo de impedância da linha de transmissão

Impedância da carga

Ângulo do fator de potência da carga

Ângulo do fator de potência de geração

Vetor de variação incremental de potência ativa

Vetor de variação incremental de potência reativa

Vetor de variação incremental do módulo da tensão nodal

Vetor de variação incremental do ângulo da tensão nodal

Matriz jacobiana

Matriz obtida a partir das sub-matrizes particionadas de $\boldsymbol{J}$

Quadrado da injeção de potência aparente na barra em análise $i$

Quadrado da estimativa da máxima injeção de potência aparente na barra em análise $i$

Indicador de distância entre $\left|\dot{S}_{i}\right|^{2}$ e $\left|\dot{S}_{i o}^{2}\right|$

Quadrado da estimativa de máxima potência aparente considerando o restante do sistema

Indicador de distância entre $\left|\dot{S}_{m}\right|^{2}$ e $\left|\dot{S}_{i o}^{2}\right|$

Vetor gradiente do fluxo de potência ativa

Vetor gradiente do fluxo de potência reativa

Ângulo entre os gradientes dos fluxos de potência ativa e reativa

Margem normalizada para a barra $i$, em percentual

Matriz jacobiana reduzida

Matriz diagonal de autovalores

Elemento $\boldsymbol{\Lambda}_{i \times i}$ da matriz de autovalores

Fator de participação da barra $k$ no modo $i$

Tensão de Thévenin

Impedância de Thévenin

Resistência de Thévenin

Reatância de Thévenin

Tolerância pré-especificada do método ŠVG

Parâmetro de correção do algoritmo de Corsi-Taranto

Parâmetro pré-especificado de correção no método de Corsi-

Taranto

Parcela constante da impedância de Thévenin da barra $j$ no 


$\dot{E}_{e q_{j}}$
$\dot{E}_{\text {acoplamento-j }}$
$\bar{Z}_{c j}$
$\bar{Z}_{j}$
$\dot{E}_{e q_{j}}^{\prime}$
$\theta_{e}$
$\bar{Z}_{j}^{\prime}$

método CAPS

Tensão de Thévenin da barra $j$ no método CAPS

Tensão de acoplamento da barra $j$ no método CAPS

Impedância de acoplamento da barra $j$ no método CAPS

Impedância de Thévenin da barra $j$ no método CAPS

Tensão de Thévenin da barra $j$ no método CAPS Aprimorado

Ângulo da tensão de Thévenin no método CAPS Aprimorado

Impedância de Thévenin da barra $j$ no método CAPS Aprimorado 


\section{Introdução}

Os desafios inerentes à operação e ao planejamento do sistema elétrico de potência aumentam gradativamente com o passar dos anos, devido, sobretudo, ao crescimento econômico e industrial, seguido pelo aumento do consumo de energia elétrica. Soma-se a isso, as limitações associadas à infraestrutura, dos investimentos insuficientes e das restrições ambientais, que naturalmente conduzem o sistema a uma operação cada vez mais próxima do seu limite crítico, ameaçando assim os requisitos fundamentais de confiabilidade, disponibilidade, qualidade e economia.

Neste contexto, o desenvolvimento do sistema de medição fasorial sincronizada (SMFS) representa uma tecnologia importante e promissora para o monitoramento do sistema elétrico.

Para elucidar o processo de medição fasorial, considera-se inicialmente que um conjunto de amostras são coletas a partir da forma de onda senoidal em um período da frequência nominal do sistema. Efetua-se então a conversão analógica/digital e posteriormente, o tratamento matemático das amostras obtidas. Em [1] menciona-se a existência de alguns métodos destinados a esta aplicação, dentre as quais a Transformada Discreta de Fourier (TDF) merece o devido destaque como a ferramenta mais utilizada atualmente. Uma abordagem eficiente para lidar com o monitoramento contínuo das formas de onda emprega a TDF em sua formulação recursiva [2], que possibilita a redução no esforço computacional requerido. Com a aquisição dos fasores de tensão e corrente em cada uma das fases, representa-se o sistema através da rede de sequência positiva.

Quando as tensões e correntes de sequência positiva são calculadas, é possível relacioná-las sob uma referência comum de tempo, desde que as amostras sejam obtidas precisamente no mesmo instante. Este processo é facilmente realizado em uma mesma subestação, aonde um pulso de clock pode ser distribuído para os diversos sistemas de medição. Contudo, para realizar medições fasoriais sob uma mesma referência comum em subestações geograficamente distantes, a tarefa de sincronização não é algo trivial. Ao longo dos anos, reconhecendo a relevância e o impacto das medições fasorias entre pontos remotos do sistema elétrico, diferentes meios de comunicação foram avaliados com o 
propósito de sincronizar os instantes de amostragem com a requerida precisão, tais como a rede de fibra óptica, sinais AM (Amplitude-Modulated), microondas e sinais via satélite do sistema GOES (Geostationary Operational Environmental Satellite). Contudo, a dificuldade em obter precisão para estabelecer o sincronismo necessário inviabilizou a utilização destes meios de comunicação $[1,2]$.

Um nova perspectiva foi adquirida no início da década de 90 com o sistema de posicionamento global (GPS - Global Positioning System) desenvolvido pelo Departamento de Defesa dos Estados Unidos, sendo utilizado no Oriente Médio durante a Operação Tempestade no Deserto, e que atualmente consiste de um sistema de 24 satélites distribuídos em 6 órbitas de altitude aproximada de $16.000 \mathrm{~km}$ acima da superfície terrestre [3]. Com o desenvolvimento desta tecnologia foi possível estabelecer o sincronismo entre amostras provenientes de subestações distantes com um erro menor que 1 microsegundo $(\mu \mathrm{s})$, o que corresponde a um erro de fase em torno de $0,021^{\circ}$ para o sistema operando em $60 \mathrm{~Hz}$ e, portanto, oferece uma precisão maior do que as exigidas para as aplicações concebidas atualmente [2].

\section{1}

\section{Unidade de Medição Fasorial}

A estrutura simplificada que compõe uma PMU (Phasor Measurement Unit) é apresentada na Figura 1.1. Neste esquema, as PMUs utilizam sinais sincronizados a partir de satélites do GPS, captados pelas estações receptoras que fornecem um sinal temporizado de um pulso por segundo (PPS), estabelecendo os instantes para obtenção das amostras de tensões e correntes trifásicas através dos enrolamentos secundários dos transformadores de corrente e de potencial [1]. Estas entradas analógicas são submetidas a um filtro anti-aliasing, responsável pela remoção do efeito aliasing, e um módulo de conversão analógico-digital que processa as amostras em sua forma fasorial utilizando por exemplo a TDF em sua formulação recursiva, para que então possam ser calculados pelo microprocessador os fasores de sequência positiva. 


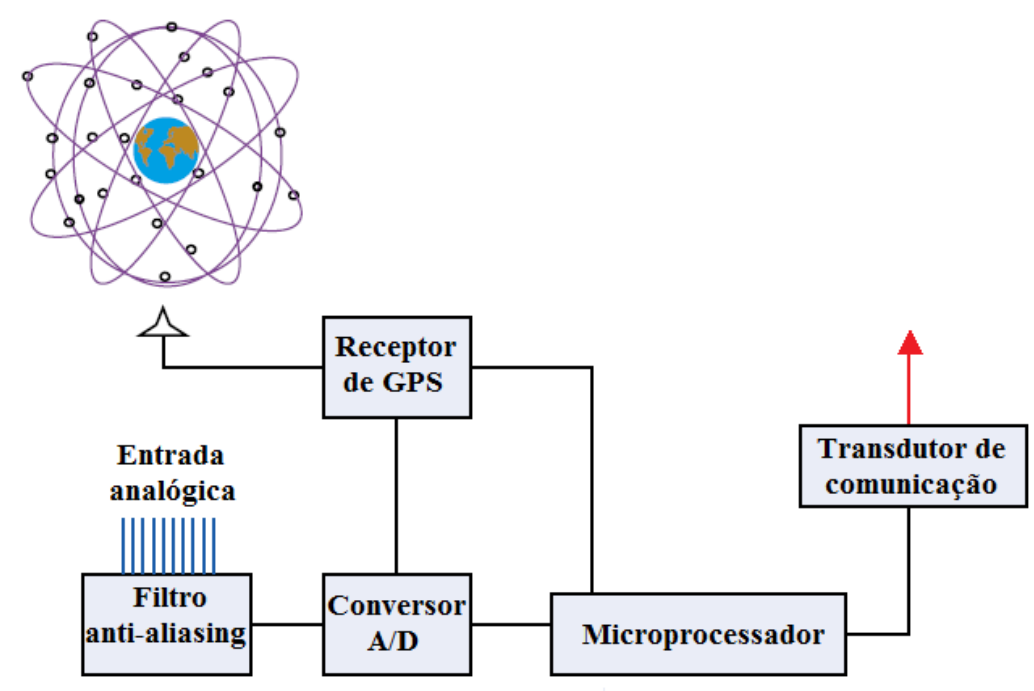

Figura 1.1: Estrutura simplificada de uma unidade de medição fasorial [3]

\section{2}

\section{Sistemas de Medição Fasorial}

Uma vez descrito o processo de medição através das PMUs, esta seção descreve sucintamente sua aplicação ao sistema elétrico, sendo integrada portanto ao sistema de medição fasorial sincronizada (SMFS).

Ao longo do tempo, diferentes alternativas acerca da arquitetura do SMFS foram analisadas considerando aspectos como a confiabilidade, eficiência e custo [1]. A estrutura simplificada mostrada na Figura 1.2 representa a arquitetura centralizada, em que os dados de cada área são enviados pelas PMUs a um único concentrador de dados. Deste modo, os elementos básicos que constituem o sistema de medição fasorial são essencialmente: as PMUs, os links de comunicação, o concentrador de dados e o GPS.

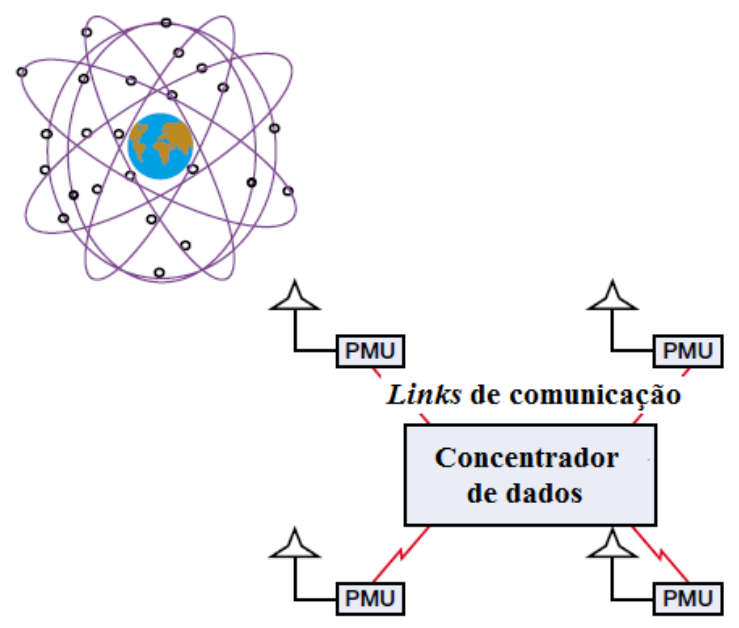

Figura 1.2: Representação genérica do sistema de medição fasorial [3] 
Após o processo de medição descrito anteriormente, os dados das diversas PMUs podem ser enviados através dos canais de comunição, empregando meios físicos ou a tecnologia sem fio (wireless), ao concentrador de dados que é responsável por sincroniza-los no tempo, armazenar e compatilhar de acordo com as aplicações desejadas.

\section{3}

\section{Objetivo e Levantamento Bibliográfico}

São extensas as aplicações que o SMFS trouxe à operação e ao planejamento do sistema elétrico, com impacto na estimação de estado, na avaliação do comportamento dinâmico do sistema e nas estratégias de controle e proteção [4].

Dentre tais contribuições, a avaliação das condições de estabilidade de tensão é destaque nesta dissertação, que se compromete a analisar os principais modelos para construção de equivalentes de rede tendo em vista a detecção do máximo carregamento. De fato, a confrontação entre os métodos não representa um objeto de estudo recente, conforme pode ser observado em $[5,6,7,8,9,10,11]$. Portanto este trabalho apresenta, além de uma análise comparativa, contribuições originais ao tema, tais como:

- Validar a utilização dos equivalentes multi-portas como índices na identificação da barra de carga crítica.

- Analisar as características do modelo baseado no Circuito Acoplado de Porta Simples Aprimorado (CAPS Aprimorado).

- Estender o método às barras de transferência e de geração.

- Investigar o desempenho do método CAPS Aprimorado sob diversas condições de carregamento e caracterizar sua sensibilidade quanto à presença de ruídos.

\section{4}

\section{Estrutura do Trabalho}

Para contemplar os objetivos mencionados, esta dissertação organiza-se da seguinte forma:

O Capítulo 1 tratou resumidamente dos principais elementos que compõem o sistema de medição fasorial, do processo de aquisição das amostras e das novas perspectivas que acompanham o seu desenvolvimento para o setor elétrico.

O Capítulo 2 apresenta uma breve descrição sobre as diferentes formas de estabilidade associadas ao sistema elétrico de potência, com maior ênfase dada 
à estabilidade de tensão. Posteriormente caracteriza-se o fenômeno a partir de um sistema equivalente de duas barras, e demonstra-se resultados importantes já conhecidos na literatura, como a existência de valores máximos de potência ativa e reativa que podem ser transferidas para uma barra de carga, e a existência de uma potência máxima absoluta (ou "Maximum Maximorum").

O Capítulo 3 prossegue com a caracterização da estabilidade de tensão, ampliando-a através da percepção de que ao fenômeno também se associa a existência de uma máxima potência ativa e reativa a ser injetada na rede através das barras terminais dos geradores e compensadores síncronos, demonstrando que a máxima carga a ser atendida pelo sistema lida com outras condições limitantes.

O Capítulo 4 revisa importantes ferramentas utilizadas para a avaliação das condições de estabilidade de tensão, como o método da matriz $\boldsymbol{D}^{\prime}$ e o método dos autovalores e autovetores. Tais índices são utilizados neste trabalho para confrontar os resultados obtidos pelos equivalentes multi-portas.

O Capítulo 5 descreve alguns dos principais métodos utilizando medições fasoriais sincronizadas encontrados na literatura para a construção do circuito equivalente de Thévenin. Escolheu-se alguns métodos baseados em medições locais e no amplo monitoramento do sistema. Algumas modificações são sugeridas tendo em vista novas aplicações para o monitoramento das barras de transferência e de geração.

O Capítulo 6 compara os métodos descritos no capítulo anterior a partir de um conjunto de análises envolvendo as abordagens estáticas e dinâmicas, com diferentes direções de carregamento do sistema, e a inserção de imprecisões nas amostras obtidas pelas PMUs.

O Capítulo 7 destaca as principais conclusões com base nos resultados obtidos, e são sugeridas novas melhorias e análises complementares àquelas apresentadas neste trabalho.

O Apêndice A detalha a demonstração das derivadas parciais que são utilizadas no método CAPS Aprimorado.

Os Apêndices B e C apresentam os dados dos sistemas utilizados na simulação. 


\section{2}

\section{Considerações Gerais sobre Estabilidade}

A estabilidade do sistema elétrico de potência pode ser caracterizada como a capacidade do sistema em manter um regime estável operativo sob condições normais, ou alcançar um estado de equilíbrio aceitável após ser submetido a dada perturbação. A estabilidade refere-se a um tema de grande abrangência e, por isto, foi divida para facilitar o seu estudo. Essa divisão, mostrada na Fig. 2.1 foi baseada primeiro na natureza do fenômeno (que pode ser angular, de frequência ou de tensão) e, em seguida, no tamanho da perturbação (pequena ou grande) e no tempo de análise (englobando a análise das ações de equipamentos de dinâmica rápida e/ou lenta) conforme descrito em [12].

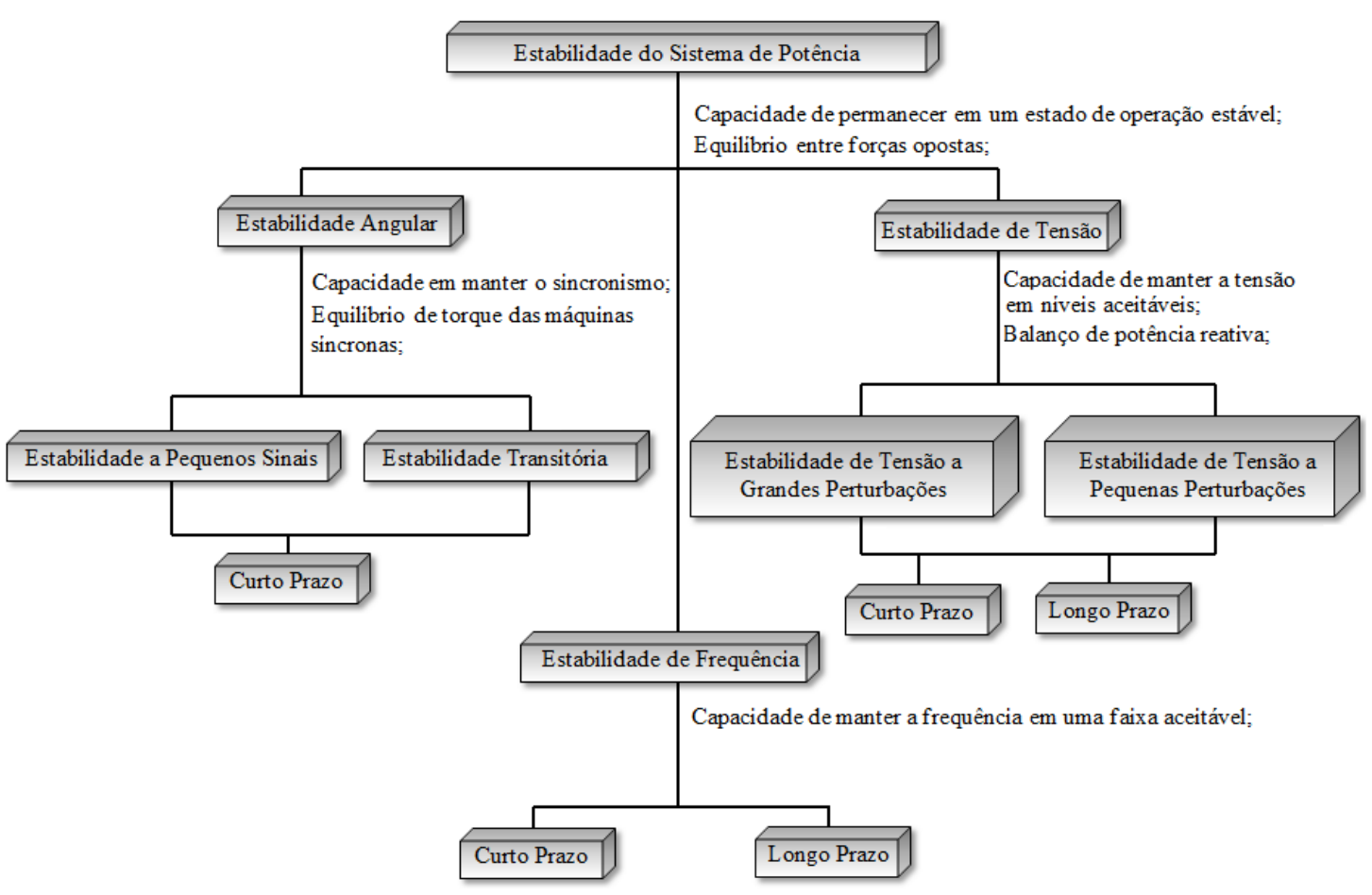

Figura 2.1: Classificação de estabilidade de sistemas de potência

A estabilidade angular refere-se à capacidade das máquinas síncronas que compõem o sistema em manterem o sincronismo em condições normais, ou após a ocorrência de uma perturbação, e pode ser categorizada em: 
(a) Estabilidade a pequenos sinais - Refere-se a capacidade do sistema em manter as máquinas em sincronismo mediante a pequenas perturbações. São exemplos de pequenas perturbações a mudança de ajuste (do inglês, setpoint) de reguladores e a variação normal da carga ao longo do dia. Estas pequenas variações permitem que a análise do caso seja realizada através da linearização das equações que descrevem o sistema sem que haja erros grosseiros no resultado.

(b) Estabilidade Transitória - É a capacidade do sistema em manter o sincronismo entre as máquinas em face de grandes perturbações, como na ocorrência de faltas em linhas de transmissão e saídas forçadas de usinas. A resposta do sistema, em decorrência a este tipo de perturbação, envolve grandes excursões no ângulo do rotor do gerador (também chamado de ângulo de carga), e é influenciado pela relação não-linear potênciaângulo. A estabilidade transitória depende depende de inúmeros fatores como, por exemplo, o cenário de operação, a severidade da pertubação, o tempo de resposta dos reguladores do sistema, entre outros.

A estabilidade de frequência, por sua vez, deve-se à capacidade do sistema manter a frequência estável quando submetido a um distúrbio severo, resultado de um significativo desbalanço entre geração e carga. Em outras palavras, depende da capacidade do sistema manter ou restaurar o equilíbrio entre geração e carga, com a mínima perda de carga não intencional. A instabilidade resultante da contínua oscilação de frequência pode conduzir ao desligamento de unidades geradoras ou de cargas.

Por fim, a estabilidade de tensão, objeto de estudo deste trabalho, é caracterizada como a capacidade do sistema manter, em níveis aceitáveis, as tensões em todas as barras, sob condições normais de operação e após eventuais perturbações. O sistema caminha para a instabilidade de tensão quando, após a ocorrência de uma perturbação como aumento de carga ou mesmo mudança nas condições do sistema, o levam para um progressivo e incontrolável declínio de tensão, conduzindo o sistema a possíveis desligamentos.

Contudo, é preciso mencionar que problemas associados à instabilidade por sobretensões já foram verificados em ao menos um sistema conforme a descrição de [13], onde se relata que uma sucessão de eventos iniciado pela ocorrência de uma falta na rede de $315 \mathrm{kV}$ do sistema elétrico canadense deu início a uma série de desligamentos, conduzindo o sistema a um cenário de instabilidade de longo prazo em que a atuação dos LTCs e dos limitadores de subexcitação dos geradores e compensadores síncronos foram responsabilizados. A instabilidade, neste caso, reside na incapacidade do sistema operar 
abaixo de determinada faixa de tensão [12].

Assim, o fenômeno da estabilidade de tensão pode ser classificado em:

(a) Estabilidade de tensão a grandes perturbações - Consiste na capacidade do sistema, após as devidas ações de controle, manter a tensão em níveis aceitáveis quando submetido a grandes perturbações tais como a ocorrência de falta no sistema, perda de unidades geradoras ou contingências de circuitos. Sua análise necessita de simulações envolvendo o desempenho dinâmico do sistema durante o período de alguns segundos a alguns minutos tendo em vista as interações de alguns dispositivos, tais como LTCs e os limitadores de corrente de campo dos geradores.

(b) Estabilidade de tensão a pequenas perturbações - Consiste na capacidade do sistema manter a tensão em níveis aceitáveis, quando submetido a pequenas perturbações tais como variações incrementais de carga. Para sua análise, mostra-se eficiente a abordagem de natureza estática, baseada apenas nas equações algébricas que capturam o estado operativo do sistema em diversos instantes. Isto permite determinar, por exemplo, a margem do sistema e identificar fatores que possuem grande influência na estabilidade.

O fenômeno associado à estabilidade de tensão pode variar entre alguns segundos a dezenas de minutos. A estabilidade de curto prazo corresponde ao período de estudo de alguns segundos, lidando com o conjunto de equações diferenciais que modelam o sistema. Deste modo, sua análise envolve a dinâmica de componentes de carga de ações rápidas como motores de indução, cargas eletronicamente controladas e sistemas HVDC.

Por sua vez, a estabilidade de longo prazo envolve um período de estudo de alguns minutos, e contempla a atuação de dispositivos de ações lentas, tais como mudança de tape dos transformadores e limitadores de corrente de sobre-excitação dos geradores.

\section{1}

\section{Caracterização da Estabilidade de Tensão}

A caracterização do fenômeno da estabilidade de tensão pode ser avaliada em um sistema simples de duas barras. Este sistema pode representar o circuito equivalente de Thévenin "visto" pela barra de carga em análise em dado ponto de operação.

O teorema de Thévenin encontra aplicações na teoria de circuitos lineares, e sua utilidade se verifica ao analisarmos o comportamento de um elemento variável em particular, a carga, desde que todos os demais elementos do circuito 
sejam fixos. Para isto, o sistema é substuído pelo circuito equivalente dotado de uma fonte de tensão $\dot{E}_{t h}$ em série com a impedância $\bar{Z}_{t h}$.

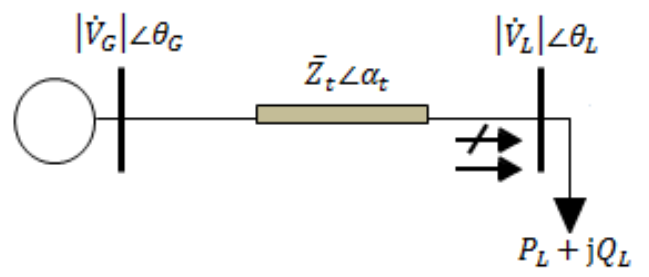

Figura 2.2: Potência ativa e reativa drenada pela carga no sistema de duas barras

Por ora, não representa objeto de preocupação a estimação dos parâme$\operatorname{tros} \dot{E}_{t h}$ e $\bar{Z}_{t h}$, mas a caracterização do fenômeno de estabilidade de tensão. Deste modo, com base no sistema da Figura 2.2, é possível escrever as seguintes equações de fluxo de carga:

$$
\begin{aligned}
& P_{L}=-\frac{\left|\dot{V}_{L}\right|^{2}}{\left|\bar{Z}_{t}\right|} \cos \alpha_{t}+\frac{\left|\dot{V}_{L}\right|\left|\dot{V}_{G}\right|}{\left|\bar{Z}_{t}\right|} \cos \left(\theta_{L G}+\alpha_{t}\right) \\
& Q_{L}=-\frac{\left|\dot{V}_{L}\right|^{2}}{\left|\bar{Z}_{t}\right|} \sin \alpha_{t}+\frac{\left|\dot{V}_{L}\right|\left|\dot{V}_{G}\right|}{\left|\bar{Z}_{t}\right|} \sin \left(\theta_{L G}+\alpha_{t}\right)
\end{aligned}
$$

Através das equações (2.1) e (2.2), propõe-se mostrar, entre outros aspectos, que a máxima potência ativa consumida pela carga, considerando o módulo da tensão na carga $\left|\dot{V}_{L}\right|$ constante, ocorre em uma defasagem angular $\theta_{L G}=-\alpha_{t}$. Para isto, deriva-se parcialmente $P_{L}$ em relação à defasagem angular entre a barra de carga e a barra de geração, donde segue:

$$
\begin{gathered}
\frac{\partial P_{L}}{\partial \theta_{L G}}=-\frac{\left|\dot{V}_{L}\right|\left|\dot{V}_{G}\right|}{\left|\bar{Z}_{t}\right|} \sin \left(\theta_{L G}+\alpha_{t}\right)=0 \therefore \\
\theta_{L G}=-\alpha_{t}
\end{gathered}
$$

A derivada de segunda ordem de (2.3) confirma se tratar de um ponto de máximo, pois:

$$
\frac{\partial^{2} P_{L}}{\partial \theta_{L G}^{2}}=-\frac{\left|\dot{V}_{L}\right|\left|\dot{V}_{G}\right|}{\left|\bar{Z}_{t}\right|} \cos \left(\theta_{L G}+\alpha_{t}\right)<0
$$

Observa-se ainda que o aumento gradativo de $\left|\dot{V}_{L}\right|$ através do adequado suporte de potência reativa produz correspondente aumento de $P_{L}$, e este valor é máximo, conforme demonstrado em (2.3), quando a defasagem angular entre a barra de carga e a barra de geração iguala-se a $-\alpha_{t}$. No entanto, tal crescimento é limitado pela própria rede, e independente de uma capacidade 
infinita de compensação de potência reativa na barra de carga. A potência máxima é obtida quando:

$$
\left|\dot{V}_{L}\right|=\frac{\left|\dot{V}_{G}\right|}{2 \cos \alpha_{t}}
$$

Assim, para valores de $\left|\dot{V}_{L}\right|$ acima de (2.4) não há como produzir aumento em $P_{L}$, e o consumo de potência ativa pela carga começa a decrescer, demonstrando a existência de máxima potência ativa a ser transferida, que será discutida detalhadamente na Seção 2.4. Isto também pode ser observado na Figura 2.3, simulando o aumento de carga em função da defasagem angular para diferentes valores de tensão na barra de carga.

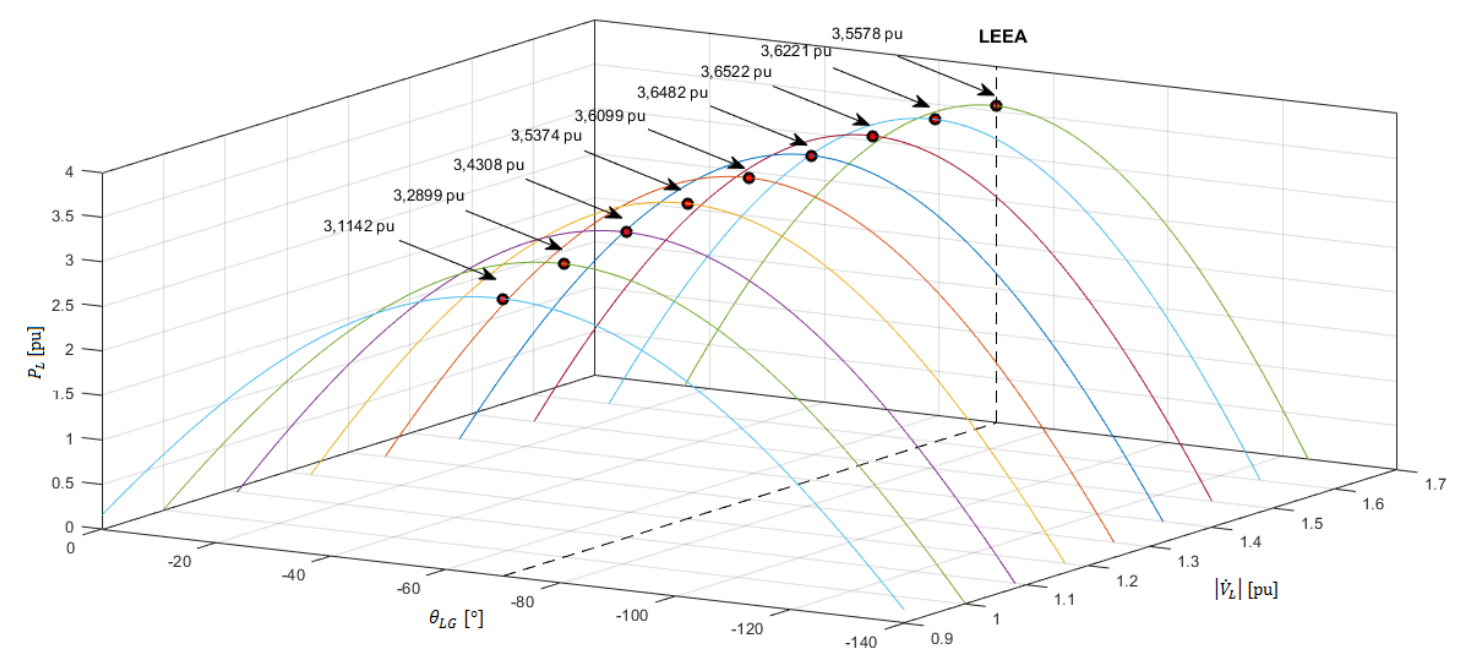

Figura 2.3: Curva $\theta \times P$ para diferentes valores de tensão na barra de carga

\section{2}

\section{Curvas $P_{L}, Q_{L}$ e $\phi$ Constantes}

Considerando as equações de fluxo de carga apresentadas, é possível mostrar no plano $\theta \times|\dot{V}|$ as soluções de tensão para valores especificados de $P_{L}$ e $Q_{L}$ constantes. Para tanto, considera-se como exemplo o sistema apresentado na Figura 2.4.

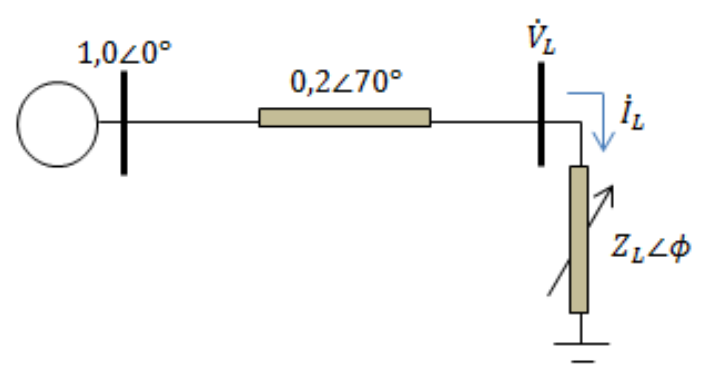

Figura 2.4: Sistema ilustrativo 
Na Figura 2.5 são mostrados os isocontornos para diferentes valores de $P_{L}$ e $Q_{L}$ constantes, em que se pode observar que o aumento da potência ativa consumida $P_{L}$ produz curvas mais fechadas de menor perímetro, convergindo para um ponto específico de máxima potência. A curva de $Q_{L}$, por sua vez, fecha-se partindo de valores de $Q_{L}$ mais capacitivos para valores mais indutivos, indicando a existência de uma máxima potência indutiva a ser alimentada pelo sistema. Observa-se também o limite de estabilidade estática angular (LEEA) que divide o plano nas regiões estável e instável de operação, tendo como lugar geométrico, a reta definida por $\theta_{L G}=-\alpha_{t}$.

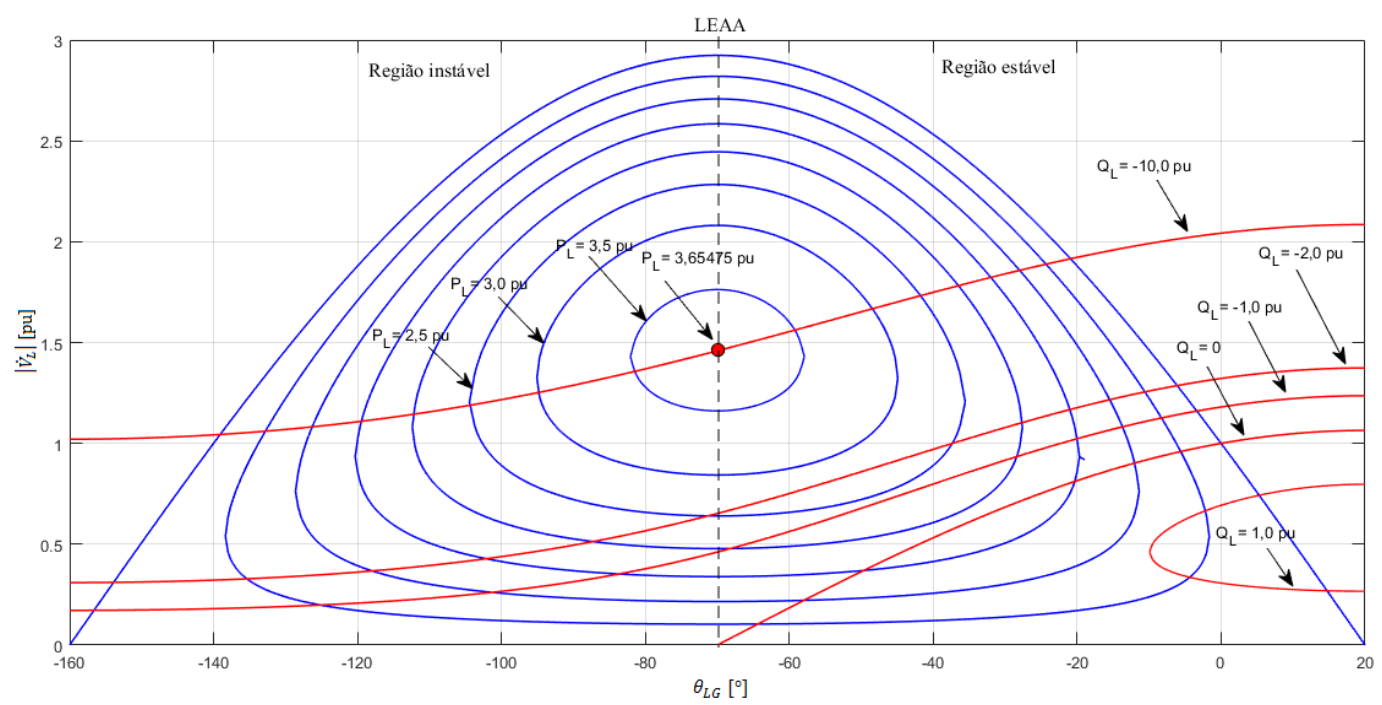

Figura 2.5: Gráfico representando o lugar geométrico das soluções de tensão para $P_{L}$ e $Q_{L}$ constantes

Destaca-se que, para uma dada carga com ângulo do fator de potência $\phi$, os isocontornos intersectam-se em dois pontos, indicando a existência de uma dupla solução de tensão para atender a carga. No entanto, com o aumento da potência consumida, mantendo-se o fator de potência constante, as curvas tornam-se mais fechadas e as soluções de tensão caminham no sentido de aproximarem-se. É plausível concluir então que na máxima potência a solução de tensão seja única, e este ponto corresponde ao limiar no qual, para valores além desta potência máxima, não haverá solução de tensão. Dito de outro modo, este ponto corresponde à máxima potência da carga $\dot{S}_{L}^{\text {máx }}$ a ser atendida pelo sistema.

A Figura 2.6 ilustra o processo descrito, segundo o qual o sistema caminha para a inexistência de solução de tensão para valores acima da máxima potência, sendo o ângulo do fator de potência da carga $\phi=45^{\circ}$. Interessante ressaltar que a curva $\phi$ constante mostrada na Figura 2.6 pode ser obtida no 
plano $\theta \times|\dot{V}|$ tomando-se:

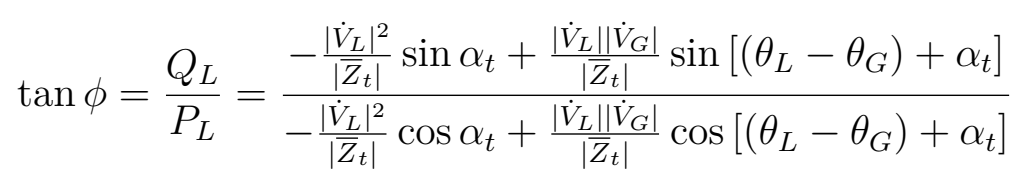

de onde segue, isolando $\left|\dot{V}_{L}\right|$ :

$$
\left|\dot{V}_{L}\right|=\frac{\left|\dot{V}_{G}\right|\left\{\sin \left[\left(\theta_{L}-\theta_{G}\right)+\alpha_{t}\right]-\cos \left[\left(\theta_{L}-\theta_{G}\right)+\alpha_{t}\right] \tan \phi\right\}}{\sin \alpha_{t}-\cos \alpha_{t} \tan \phi}
$$

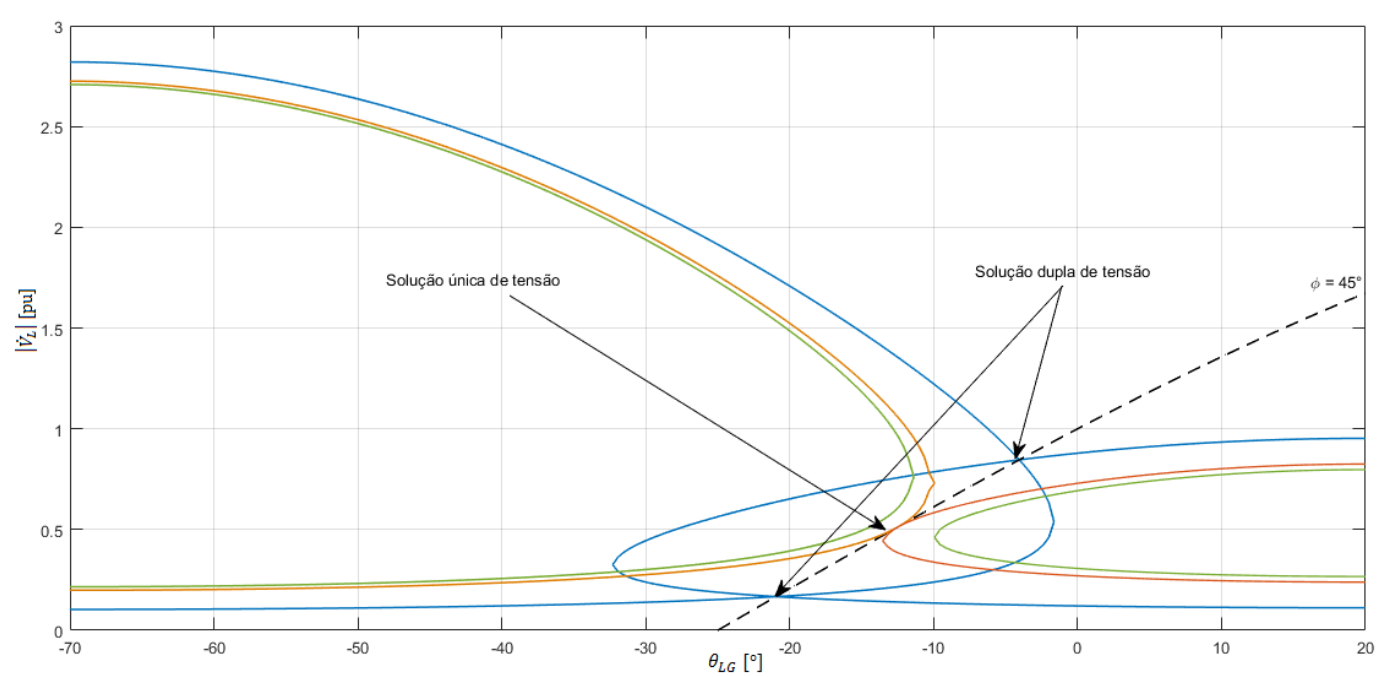

Figura 2.6: Avaliação da existência de soluções de tensão para diferentes patamares de carga com $\phi=45^{\circ}$

\section{3}

\section{Avaliação da Máxima Transferência de Potência}

Do exposto, chega-se a uma das conhecidas propriedades da teoria de circuitos elétricos, que se deve à existência da máxima transferência de potência a ser entregue à carga, ou o "Teorema da máxima transferência de potência". Então, para uma dada carga $\bar{Z}_{L}$, a potência aparente $\dot{S}_{L}$ é obtida pela expressão:

$$
\dot{S}_{L}=\dot{V}_{L} \dot{I}_{L}^{*}=\left(\bar{Z}_{L} \dot{I}_{L}\right) \dot{I}_{L}^{*}=\bar{Z}_{L}\left|\dot{I}_{L}\right|^{2}
$$

onde:

$$
\left|\dot{I}_{L}\right|=\frac{\left|\dot{V}_{G}\right|}{\sqrt{\left[\left(\left|\bar{Z}_{t}\right| \cos \alpha_{t}+\left|\bar{Z}_{L}\right| \cos \phi\right)^{2}+\left(\left|\bar{Z}_{t}\right| \sin \alpha_{t}+\left|\bar{Z}_{L}\right| \sin \phi\right)^{2}\right]}}
$$

portanto, substituindo (2.8) em (2.7), considerando as identidades trigonométricas, obtém-se: 


$$
\dot{S}_{L}=\frac{\bar{Z}_{L}\left|\dot{V}_{G}\right|^{2}}{\left|\bar{Z}_{t}\right|^{2}+\left|\bar{Z}_{L}\right|^{2}+2\left|\bar{Z}_{t}\right|\left|\bar{Z}_{L}\right| \cos \left(\phi-\alpha_{t}\right)}
$$

Para demonstrar a existência de uma potência $\dot{S}_{L}^{\text {máx }}$ para cada ângulo do fator de potência $\phi$, toma-se a derivada parcial de $\dot{S}_{L}$ em relação à $\left|\bar{Z}_{L}\right|$ igual a zero, ou seja:

$$
\frac{\partial \dot{S}_{L}}{\partial\left|\bar{Z}_{L}\right|}=\frac{\left|\dot{V}_{G}\right|^{2}\left(\left|\bar{Z}_{t}\right|^{2}-\left|\bar{Z}_{L}\right|^{2}\right)}{\left[\left|\bar{Z}_{t}\right|^{2}+\left|\bar{Z}_{L}\right|^{2}+2\left|\bar{Z}_{t}\right|\left|\bar{Z}_{L}\right| \cos \left(\phi-\alpha_{t}\right)\right]^{2}}=0
$$

desde que $\left[\left|\dot{Z}_{t}\right|^{2}+\left|\bar{Z}_{L}\right|^{2}+2\left|\bar{Z}_{t}\right|\left|\bar{Z}_{L}\right| \cos \left(\phi-\alpha_{t}\right)\right] \neq 0$, então:

$$
\begin{gathered}
\left|\dot{V}_{G}\right|\left(\left|\bar{Z}_{L}\right|^{2}-\left|\bar{Z}_{t}\right|^{2}\right)=0 \therefore \\
\left|\bar{Z}_{L}\right|=\left|\bar{Z}_{t}\right|
\end{gathered}
$$

Verifica-se que a identidade (2.11) constitui uma solução de máximo através da derivada de segunda ordem, pois:

$$
\frac{\partial^{2} \dot{S}_{L}}{\partial\left|\bar{Z}_{L}\right|^{2}}<0
$$

Portanto, demonstra-se que a máxima transferência de potência $\left|S_{L}^{\text {máx }}\right|$ é observada quando $\left|\bar{Z}_{L}\right|=\left|\bar{Z}_{t}\right|$, e será dada por:

$$
\left|\dot{S}_{L}^{\text {máx }}\right|=\frac{\left|\dot{V}_{G}\right|^{2}}{2\left|\bar{Z}_{t}\right|\left[1+\cos \left(\phi-\alpha_{t}\right)\right]}
$$

logo, para a máxima potência ativa e reativa:

$$
\begin{aligned}
P_{L}{ }^{\text {máx }} & =\frac{\left|\dot{V}_{G}\right|^{2} \cos \phi}{2\left|\bar{Z}_{t}\right|\left[1+\cos \left(\phi-\alpha_{t}\right)\right]} \\
Q_{L}{ }^{\text {máx }} & =\frac{\left|\dot{V}_{G}\right|^{2} \sin \phi}{2\left|\bar{Z}_{t}\right|\left[1+\cos \left(\phi-\alpha_{t}\right)\right]}
\end{aligned}
$$

Um conceito importante refere-se a margem de carregamento da barra em análise, que expressa o valor em percentual de proximidade do ponto de operação em análise, tendo em vista a máxima transferência de potência:

$$
\text { Margem }=\left(\frac{\left|\dot{S}_{L}^{\text {máx }}\right|-\left|\dot{S}_{L}\right|}{\left|\dot{S}_{L}^{\text {máx }}\right|}\right) \times 100 \%
$$

A solução de tensão $\dot{V}_{L}$ para a condição de máxima transferência, acima descrita, pode ser obtida tomando:

$$
P_{L}^{\text {máx }}=\left|\dot{V}_{L}\right|^{c}\left|\dot{I}_{L}\right| \cos \phi \Rightarrow\left|\dot{V}_{L}\right|^{c}=\frac{P_{L}^{\operatorname{máx}}}{\left|\dot{I}_{L}\right| \cos \phi}
$$


substituindo (2.8) e (2.13) em (2.16), chega-se a:

$$
\left|\dot{V}_{L}\right|^{c}=\frac{\left|\dot{V}_{G}\right|}{\sqrt{2\left[1+\cos \left(\phi-\alpha_{t}\right)\right]}}=\frac{\left|\dot{V}_{G}\right|}{\sqrt{4\left[\frac{1+\cos \left(\phi-\alpha_{t}\right)}{2}\right]}}=\frac{\left|\dot{V}_{G}\right|}{2 \cos \left(\frac{\phi-\alpha_{t}}{2}\right)}
$$

em contrapartida, aplicando o divisor de tensão:

$$
\dot{V}_{L}^{c}=\left(\frac{\bar{Z}_{t} \dot{V}_{G}}{\bar{Z}_{L}+\bar{Z}_{t}}\right)_{\left|\bar{Z}_{L}\right|=\left|\bar{Z}_{t}\right|}
$$

avaliando a componente real de $\dot{V}_{L}^{c}$ :

$$
\left|\dot{V}_{L}\right|^{c} \cos \theta_{L G}^{c}=\frac{\left|\dot{V}_{G}\right|}{2} \Rightarrow\left|\dot{V}_{L}\right|^{c}=\frac{\left|\dot{V}_{G}\right|}{2 \cos \theta_{L G}^{c}}
$$

por fim, comparando (2.17) e (2.19):

$$
\cos \theta_{L G}^{c}=\cos \left(\frac{\phi-\alpha_{t}}{2}\right)
$$

$\log \mathrm{:}$

$$
\theta_{L G}^{c}=\frac{\phi-\alpha_{t}}{2}
$$

Tomando como exemplo o sistema da Figura 2.4, $\operatorname{com} \phi=45^{\circ}$, pode-se, a partir das equações $(2.12),(2.17)$ e (2.20) calcular os valores de $\left|S_{L}^{\text {máx }}\right|$ e a solução de tensão $\dot{V}_{L}^{c}$. Assim:

$$
\begin{gathered}
\left|\dot{S}_{L}^{\text {máx }}\right|=\frac{1,0^{2}}{0,4\left[1+\cos \left(-25^{\circ}\right)\right]}=1,311 \quad[\mathrm{pu}] \\
\dot{V}_{L}^{c}=\frac{1,0}{2 \cos \left(-12,5^{\circ}\right)} \angle-12,5^{\circ}=0,512 \angle-12,5^{\circ} \quad[\mathrm{pu}]
\end{gathered}
$$

validando a análise graficamente: 


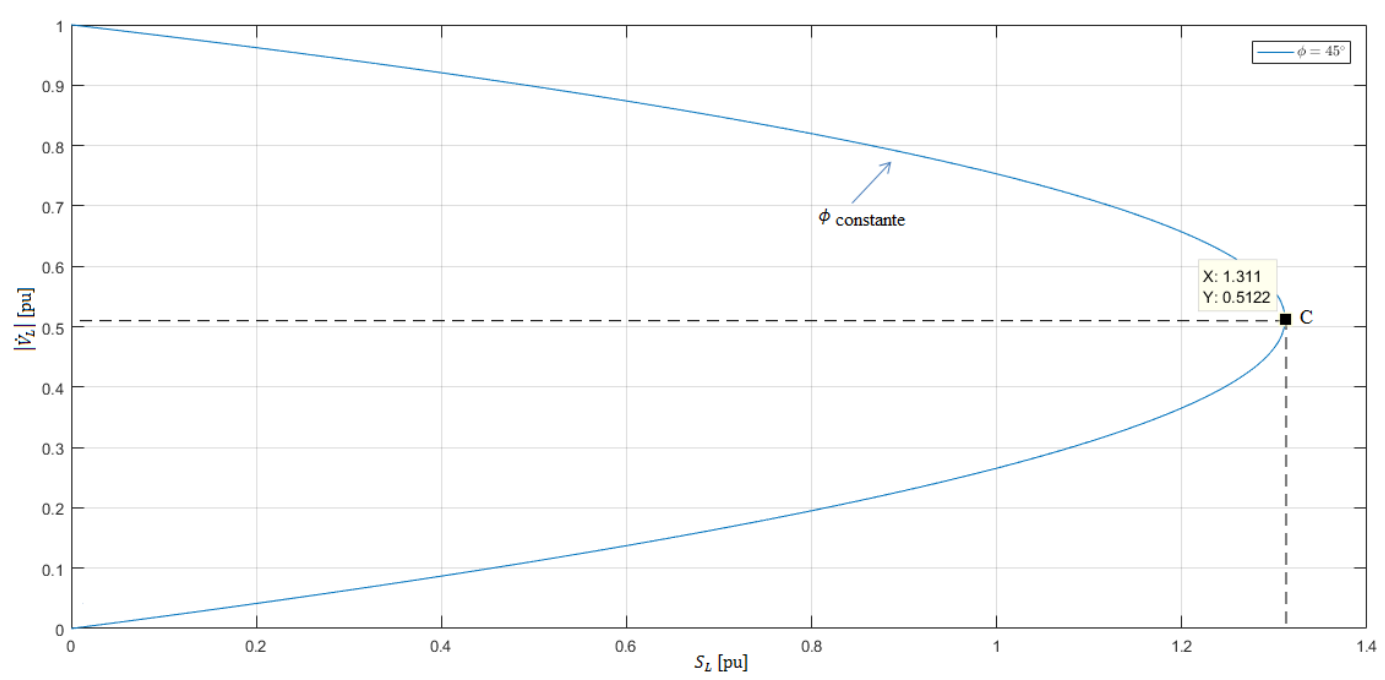

Figura 2.7: Curva $\left|\dot{S}_{L}\right| \times\left|\dot{V}_{L}\right| \operatorname{com} \phi=45^{\circ}$

Convém neste momento definir o chamado limite de estabilidade de tensão (LET) como sendo o lugar geométrico das soluções de tensão $\left(\theta_{L G}^{c},\left|\dot{V}_{L}\right|^{c}\right)$ no plano $\theta \times|\dot{V}|$, ou das soluções $\left(\left|\dot{S}_{L}\right|^{\text {máx }},\left|\dot{V}_{L}\right|^{c}\right)$ no plano $|\dot{S}| \times|\dot{V}|$ correspondentes à máxima potência ativa e reativa que podem chegar à barra de carga. Cada ponto, isto é, cada solução, corresponde a um fator de potência. Além disto, como se observa na Figura 2.8, o LET divide o plano em duas regiões, sendo a região superior dada pelo conjunto dos pontos de operação ditos "normais" e a região inferior dada pelo conjunto de pontos de operação "anormais", onde as ações de controle podem ter efeito oposto ao esperado.

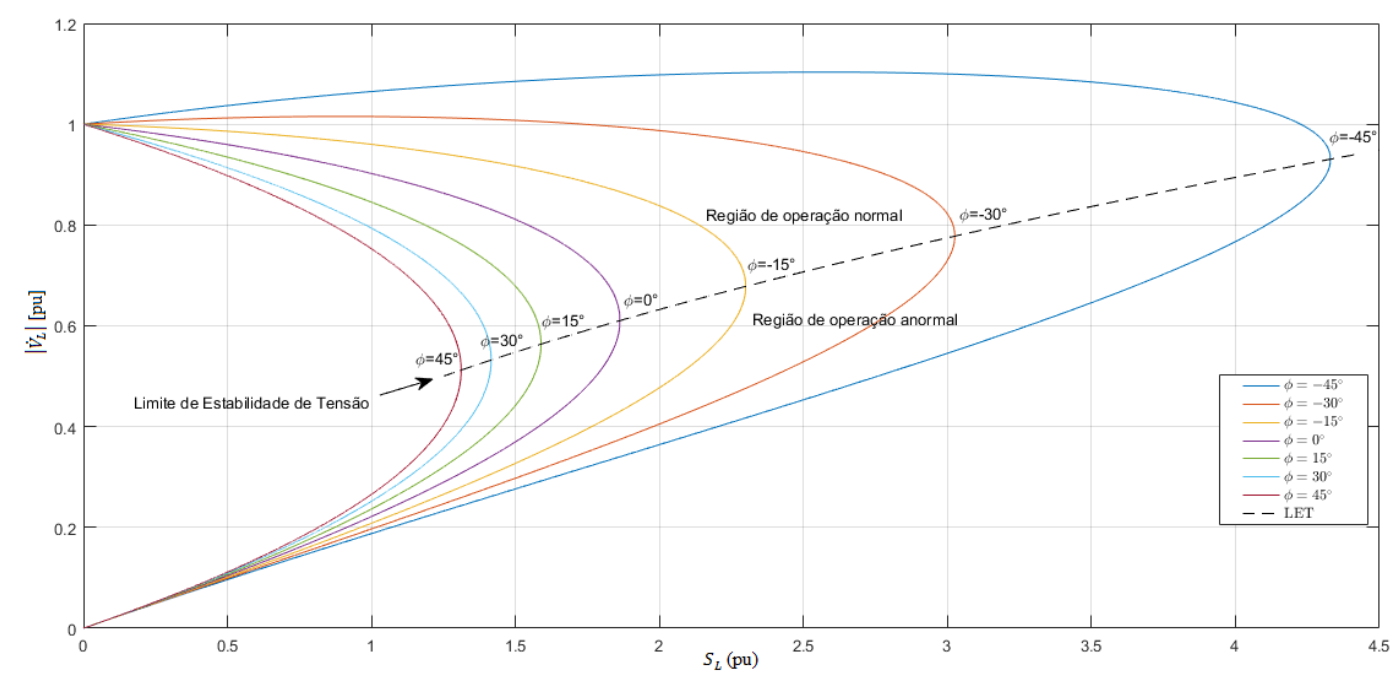

Figura 2.8: Curva $\left|\dot{S}_{L}\right| \times\left|\dot{V}_{L}\right|$ para diferentes fatores de potência 


\section{4}

\section{Demonstração da Existência de $P_{L}^{\text {máx,máx }}$}

Demonstrou-se a existência de uma máxima potência ativa a ser transferida para a carga, uma para cada fator de potência. Ainda assim, é possível demonstrar que entre as máximas encontra-se a máxima absoluta, ou "Maximum Maximorum". Tomando a equação (2.13), e derivando-a parcialmente em relação à $\phi$, busca-se determinar para qual fator de potência encontra-se $P_{L}^{\text {máx,máx }}$

$\frac{\partial P_{L}^{\text {máx }}}{\partial \phi}=\frac{-2\left|\dot{V}_{G}\right|^{2}\left|\bar{Z}_{t}\right| \sin \phi\left[1+\cos \left(\phi-\alpha_{t}\right)\right]+2\left|\dot{V}_{G}\right|^{2}\left|\bar{Z}_{t}\right| \sin \left(\phi-\alpha_{t}\right) \cos \phi}{4\left|\bar{Z}_{t}\right|^{2}\left[1+\cos \left(\phi-\alpha_{t}\right)\right]^{2}}$

igualando (2.21) a zero, obtém-se:

$$
-\sin \phi+\underbrace{\sin \left(\phi-\alpha_{t}\right) \cos \phi-\sin \phi \cos \left(\phi-\alpha_{t}\right)}_{\sin \left[\phi-\left(\phi-\alpha_{t}\right)\right]}=0
$$

portanto:

$$
-\sin \phi+\sin \left(-\alpha_{t}\right)=0
$$

como $\sin \left(-\alpha_{t}\right)=-\sin \alpha_{t h}$, então:

$$
\sin \phi=-\sin \alpha_{t} \Rightarrow \phi=-\alpha_{t}
$$

Tomando, de maneira análoga, a derivada de segunda ordem, constata-se que a solução constitui um ponto de máximo para $P_{L}^{\text {máx }}$. Portanto, em síntese, verificou-se a existência de uma máxima potência ativa $P_{L}^{\text {máx }}$ a ser entregue à carga, uma para cada fator de potência que ocorre quando $\left|\bar{Z}_{L}\right|=\left|\bar{Z}_{t}\right|$. Ademais, entre todas as máximas, destaca-se uma $P_{L}^{\text {máx,máx }}$, ou "Maximum Maximorum", que ocorre quando $\bar{Z}_{L}=\bar{Z}_{t}^{*}$.

Para o sistema exemplificado, substituindo $\bar{Z}_{L}=\bar{Z}_{t}^{*}$ em (2.13), obtém-se $P_{L}=3,6547[\mathrm{pu}]$, e portanto $Q_{L}=-10,0412[\mathrm{pu}]$ capacitivo. Na Figura 2.9 é possível identificar as máximas potências a serem transmitidas para cada fator de potência, e a curva definida pelo LET em que a solução de tensão é única. 


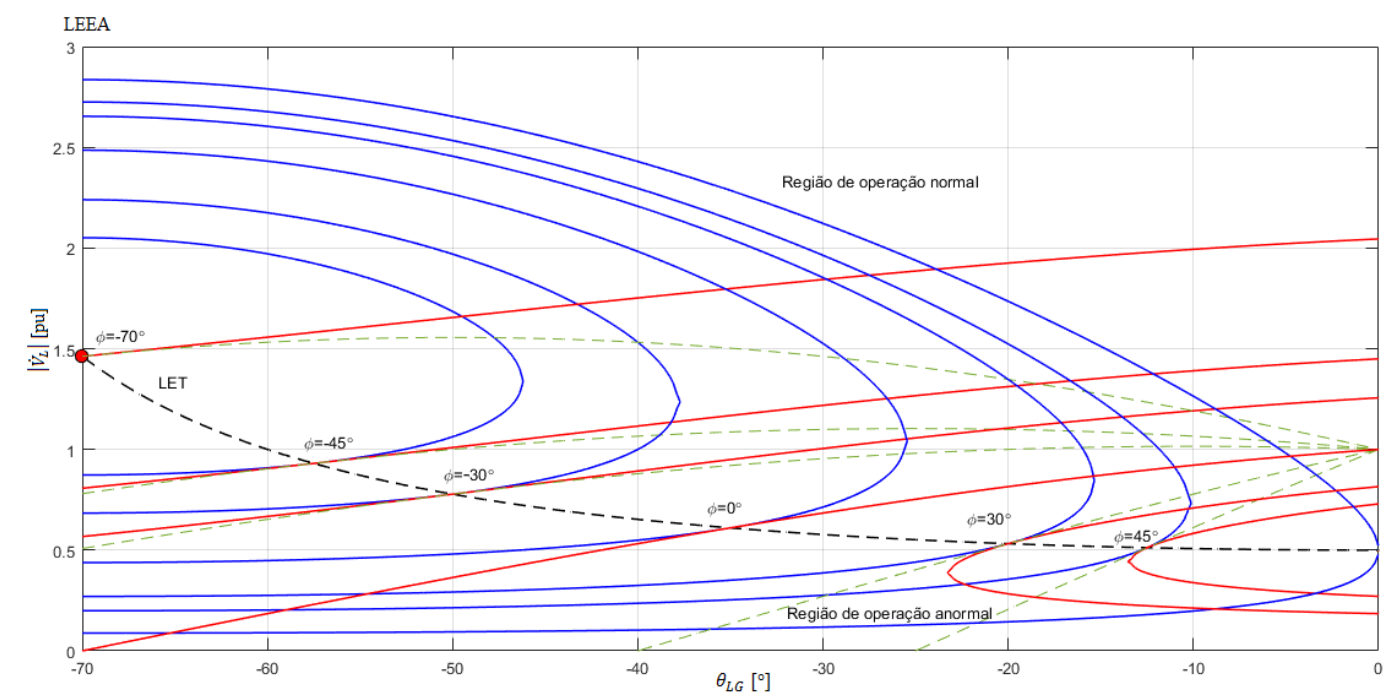

Figura 2.9: Lugar geométrico das soluções de tensão para diferentes patamares de carga constante

\section{5}

\section{Conclusões}

Até este ponto foi possível observar que:

1. A estabilidade de tensão é caracterizada pela existência de uma máxima potência ativa e reativa, uma para da cada fator de potência, que pode ser transferida pelo sistema à carga. Esta condição independe das limitações impostas ao sistema, como perdas térmicas, níveis de tensão nas barras, ou capacidade de geração. Além disto, constatou-se a existência de uma potência ativa máxima absoluta, ou "Maximum Maximorum", que independe da infinita capacidade de compensação de potência reativa.

2. Através do congestionamento do sistema de transmissão, e a utilização de compensação de potência reativa visando aumentar a tranferência de grandes blocos de potência, o fenômeno da estabilidade de tensão veio a se tornar um problema para a operação do sistema elétrico, podendo se manifestar na faixa de operação normal.

3. Em todos os casos em que se verificam problemas associados à estabilidade de tensão, a insuficiência no suporte de potência reativa não pode ser unicamente responsabilizada, a existência de compensação excessiva também pode conduzir o sistema à instabilidade. 


\section{3}

\section{Avaliação das Barras de Geração}

Anteriormente no Capítulo 2, ficou caracterizado o fenômeno da estabilidade de tensão a partir de uma barra de carga em análise. No entanto, sabe-se que o fenômeno associado à estabilidade de tensão manifesta-se também pela existência de uma máxima potência ativa e reativa que pode ser injetada na rede por geradores e compensadores síncronos para dado fator de potência, através da barra terminal. A abordagem apresentada em [14], e revisitada brevemente neste capítulo, será incorporada neste trabalho para justificar a necessidade de metodologias de estimação dos equivalentes de rede considerando a utilização de medições fasoriais para a detecção da máxima potência injetada na rede.

\section{1}

\section{Caracterização da Estabilidade de Tensão a partir da Barra de Geração}

Ao considerar o sistema de duas barras mostrado na Figura 3.1, é possível, de modo análogo ao realizado para a barra de carga, obter as expressões relativas às potências ativa e reativa injetadas na rede através da barra terminal.

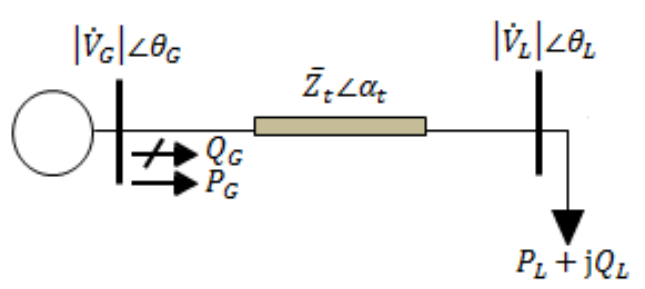

Figura 3.1: Potência ativa e reativa injetada pela barra terminal no sistema de duas barras

$$
\begin{aligned}
P_{G} & =\frac{\left|\dot{V}_{G}\right|^{2}}{\left|\bar{Z}_{t}\right|} \cos \alpha_{t}-\frac{\left|\dot{V}_{G}\right|\left|\dot{V}_{L}\right|}{\left|\bar{Z}_{t}\right|} \cos \left(\theta_{G L}+\alpha_{t}\right) \\
Q_{G} & =\frac{\left|\dot{V}_{G}\right|^{2}}{\left|\bar{Z}_{t}\right|} \sin \alpha_{t h}-\frac{\left|\dot{V}_{G}\right|\left|\dot{V}_{L}\right|}{\left|\bar{Z}_{t}\right|} \sin \left(\theta_{G L}+\alpha_{t}\right)
\end{aligned}
$$

A tangente do ângulo do fator de potência de geração $\phi_{G}$ será dada por: 


$$
\tan \phi_{G}=\frac{Q_{G}}{P_{G}}=\frac{\frac{\left|\dot{V}_{G}\right|^{2}}{\left|\bar{Z}_{t}\right|} \sin \alpha_{t}-\frac{\left|\dot{V}_{G} \| \dot{V}_{L}\right|}{\left|\bar{Z}_{t}\right|} \sin \left(\theta_{G L}+\alpha_{t}\right)}{\frac{\left|\dot{V}_{G}\right|^{2}}{\left|\bar{Z}_{t}\right|} \cos \alpha_{t}-\frac{\left|\dot{V}_{G}\right|\left|\dot{V}_{L}\right|}{\left|\bar{Z}_{t}\right|} \cos \left(\theta_{G L}+\alpha_{t}\right)}
$$

isolando $\left|\dot{V}_{G}\right|$ em (3.3) é possível traçar a curva de tensão no gerador no plano $\theta \times|\dot{V}|$ para um valor de $\phi_{G}$ constante fazendo:

$$
\left|\dot{V}_{G}\right|=\frac{\left|\dot{V}_{L}\right|\left\{\sin \left[\theta_{G L}+\alpha_{t}\right)-\cos \left(\theta_{G L}+\alpha_{t}\right) \tan \phi_{G}\right\}}{\sin \alpha_{t}-\tan \phi_{G} \cos \alpha_{t}}
$$

\section{2}

\section{Tensão Crítica na Barra Terminal}

Em um cenário de máxima injeção de potência ativa $P_{G}$ e reativa $Q_{G}$, mantendo-se o ângulo do fator de potência $\phi_{G}$ constante, os vetores grandientes $\nabla \overrightarrow{P_{G}}$ e $\nabla \overrightarrow{Q_{G}}$ encontram-se alinhados de tal modo que, tomando a variável escalar $\lambda \in \mathbb{R}$, obtém-se:

$$
\nabla \vec{P}_{G}-\lambda \nabla \vec{Q}_{G}=0
$$

Trata-se, portanto, de uma condição de otimalidade, que consiste em maximizar $P_{G}\left(\left|\dot{V}_{G}\right|, \theta_{G}\right)$ e $Q_{G}\left(\left|\dot{V}_{G}\right|, \theta_{G}\right)$ sujeito a condição $\phi_{G}=\tan ^{-1}\left(\frac{Q_{G}}{P_{G}}\right)$ constante, então:

$$
\begin{gathered}
\frac{\partial P_{G}}{\partial \theta_{G}}-\lambda \frac{\partial Q_{G}}{\theta_{G}}=0 \\
\frac{\partial P_{G}}{\partial\left|\dot{V}_{G}\right|}-\lambda \frac{\partial Q_{G}}{\partial\left|\dot{V}_{G}\right|}=0
\end{gathered}
$$

isolando $\lambda$ em (3.7), e substituindo em (3.6), chega-se a:

$$
\left(\frac{\partial P_{G}}{\partial \theta_{G}} \frac{\partial Q_{G}}{\partial\left|\dot{V}_{G}\right|}\right)-\left(\frac{\partial P_{G}}{\partial\left|\dot{V}_{G}\right|} \frac{\partial Q_{G}}{\theta_{G}}\right)=0
$$

onde:

$$
\begin{gathered}
\frac{\partial P_{G}}{\partial \theta_{G}}=\frac{\left|\dot{V}_{G}\right|\left|\dot{V}_{L}\right|}{\left|\bar{Z}_{t}\right|} \sin \left(\theta_{G L}+\alpha_{t}\right) \\
\frac{\partial P_{G}}{\partial\left|\dot{V}_{G}\right|}=2 \frac{\left|\dot{V}_{G}\right|}{\left|\bar{Z}_{t}\right|} \cos \left(\theta_{G L}+\alpha_{t}\right)-\frac{\left|\dot{V}_{L}\right|}{\left|\bar{Z}_{t}\right|} \cos \left(\theta_{G L}+\alpha_{t}\right) \\
\frac{\partial Q_{G}}{\partial \theta_{G}}=-\frac{\left|\dot{V}_{G}\right|\left|\dot{V}_{L}\right|}{\left|\bar{Z}_{t}\right|} \cos \left(\theta_{G L}+\alpha_{t}\right) \\
\frac{\partial Q_{G}}{\partial\left|\dot{V}_{G}\right|}=2 \frac{\left|\dot{V}_{G}\right|}{\left|\bar{Z}_{t}\right|} \sin \left(\theta_{G L}+\alpha_{t}\right)-\frac{\left|\dot{V}_{L}\right|}{\left|\bar{Z}_{t}\right|} \sin \left(\theta_{G L}+\alpha_{t}\right)
\end{gathered}
$$

Substituindo (3.9),(3.10),(3.11),(3.12) em (3.8), obtém-se o valor da tensão crítica no gerador na condição de máxima injeção de potência ativa e reativa dada por: 
substituindo $\left|\dot{V}_{G}\right|^{c}$ em (3.4):

$$
\left|\dot{V}_{G}\right|^{c}=\frac{\left|\dot{V}_{L}\right|}{2 \cos \left(\theta_{G L}\right)}
$$

$$
\tan \left(2 \theta_{G L}^{c}\right)=\frac{\sin \left(-\phi_{G}+\alpha_{t}\right)}{-\cos \left(-\phi_{G}+\alpha_{t}\right)}
$$

Conforme destacado em [14], a defasagem angular crítica $2 \theta_{G L}^{c}$ pode apresentar soluções nos quatro quadrantes. No entanto, apenas uma solução é factível, estando associada à condição de máxima potência gerada e garantindo a alimentação da carga.

Ao considerar o equivalente de duas barras mostrado na Figura 2.4, tomando a impedância de transferência $\bar{Z}_{t}$ idêntica àquela apresentada no Capítulo 2, ou seja, $\bar{Z}_{t}=0,2 \angle 70^{\circ}[\mathrm{pu}]$, e, ainda, adotando $\left|\dot{V}_{L}\right|=0,95[\mathrm{pu}]$ e o ângulo do fator de potência de geração $\phi_{G}=185^{\circ}$ é possível traçar a curva $\phi_{G}$ constante no plano $S \times|\dot{V}|$. A defasagem angular crítica nestas circunstâncias será dada por:

$$
\theta_{G L}^{c}=\tan ^{-1}\left(\frac{\phi_{G}-\alpha_{t}}{2}\right)=57,5^{\circ} \text { ou } 57,5^{\circ} \pm 180^{\circ}
$$

Deve-se notar a existência de uma dupla solução angular para a equação (3.15) que, por sua vez, representam soluções analíticas, porém não implicam em uma solução fisicamente possível, pois $\theta_{G L}^{c}=237,5^{\circ}=-122,5^{\circ} \Rightarrow\left|\dot{V}_{G}\right|^{c}=$ $-0,8841[\mathrm{pu}]$. Deste modo, toma-se a solução angular de (3.15) como:

$$
\theta_{G L}^{c}=57,5^{\circ}
$$

substituindo (3.16) em (3.13):

$$
\left|\dot{V}_{G}\right|^{c}=\frac{0,95}{2 \cos \left(57,5^{\circ}\right)}=0,8841 \quad[\mathrm{pu}]
$$

A potência aparente crítica mostrada na Figura 3.2 é obtida substituindo as soluções de tensão crítica nas equações de fluxo de carga, ou seja:

$$
\dot{S}_{G}^{c}=P_{G}^{c}+j Q_{G}^{c}=P_{G}\left(\left|\dot{V}_{G}\right|^{c}, \theta_{G L}^{c}\right)+j Q_{G}\left(\left|\dot{V}_{G}\right|^{c}, \theta_{G L}^{c}\right)
$$

logo:

$$
\dot{S}_{G}^{c}=3,8928+j 0,3406 \quad[\mathrm{pu}] \Rightarrow\left|\dot{S}_{G}^{c}\right|=3,9076 \quad[\mathrm{pu}]
$$




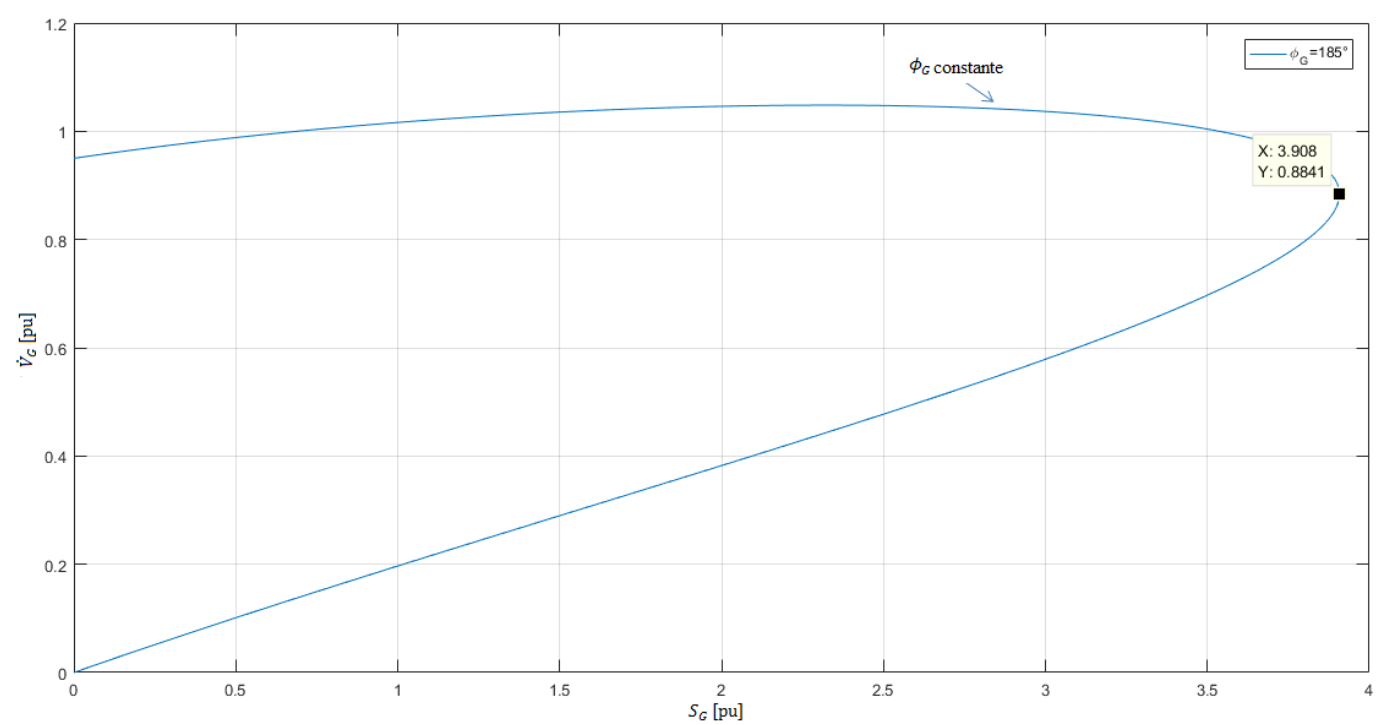

Figura 3.2: Curva $\left|\dot{S}_{G}\right| \times|\dot{V}|$ da barra de geração, com $\phi_{G}=185^{\circ}$.

As curvas $P_{G}$ e $Q_{G}$ constantes no plano $\theta \times|\dot{V}|$ são mostradas na Figura 3.3, e demonstram que o crescimento gradual de $P_{G}$ ocorre a partir de isocontornos mais internos aos mais externos ou, em outras palavras, "de dentro para fora" [14].

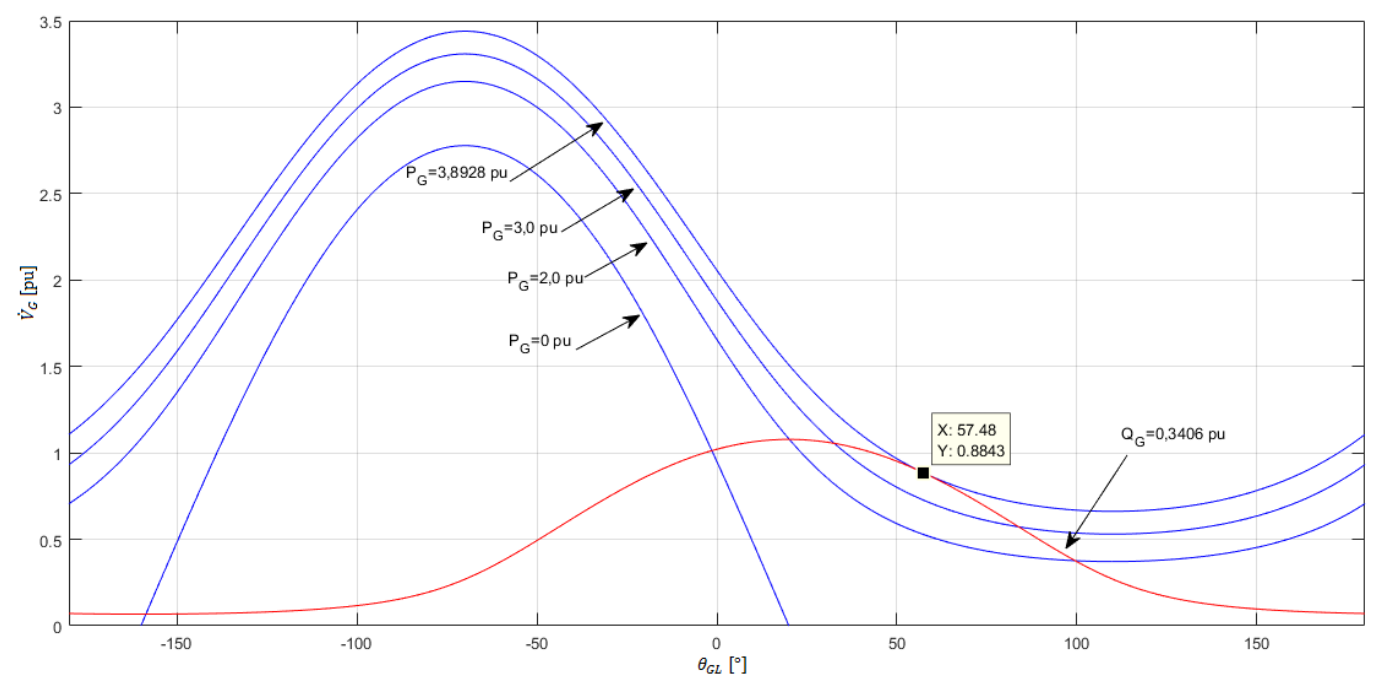

Figura 3.3: Gráfico representando o lugar geométrico das soluções de tensão para $P_{G}$ e $Q_{G}$ constantes

\section{3}

\section{Exemplos Numéricos}

Esta seção destina-se a ilustrar, através de diferentes exemplos numéricos, como a máxima carga a ser atendida pode estar associada ao máximo fluxo de 
potência que chega à barra de carga, ou pela máxima potência injetada na rede pelos geradores. Para tanto, ao assumir o ângulo do fator de potência constante na carga é possível traçar a curva $\phi$ constante no plano $P \times|\dot{V}|$ da barra de carga, e localizar o ponto de máxima potência ativa $\left(P_{L}^{\operatorname{máx}},\left|\dot{V}_{L}\right|^{c}\right)$ nomeado como ponto "1". Para tal ponto de operação obtém-se o ângulo do fator de potência de geração $\phi_{G}$, e o ponto "3" em que a barra de geração encontra-se em relação ao ponto de operação "1", ou seja, a solução $\left(S_{G},\left|\dot{V}_{G}\right|\right)$ que implica em $\left(P_{L}^{\text {máx }},\left|\dot{V}_{L}\right|^{c}\right)$ na barra de carga. Mantendo-se $\phi_{G}$ constante é traçada a curva no plano $P \times|\dot{V}|$ da barra de geração, em que se localiza o ponto "2" correspondente à estimativa de máxima injeção de potência, e cujo ponto de operação é intersectado pelo LET de geração.

\section{Caso 1 - Carga com $\phi=5,00^{\circ}$}

Para o caso 1, considera-se o ângulo do fator de potência da carga $\phi=5,00^{\circ}$, e ao traçar a curva $P \times|\dot{V}|$ da barra de carga, obtém-se a tensão crítica $\dot{V}_{L}^{c}=0,5928 \angle-32,50^{\circ}[\mathrm{pu}]$ e a potência ativa máxima $P_{L}^{\text {máx }}=1,7506$ [pu], identificados na Figura 3.4 como o ponto "1" (ver Tabela 3.1). Nestas circunstâncias, a barra de geração encontra-se no ponto "3", localizado na região superior da curva $P \times|\dot{V}|$, com a tensão igual a $1,0000 \angle 0^{\circ}[\mathrm{pu}]$, e potência gerada $\dot{S}_{G}=2,3517+j 1,8045[\mathrm{pu}]$, portanto, com o ângulo do fator de potência $\phi_{G}=217,50^{\circ}$. Construindo a curva $P \times|\dot{V}|$ para a barra de geração, identifica-se o " 2 " devido à estimativa de máxima potência gerada, e observa-se então o seu distanciamento ao "3". Portanto, conclui-se que a máxima potência entregue à barra de carga é limitada apenas pelo fluxo de potência que chega à barra. 


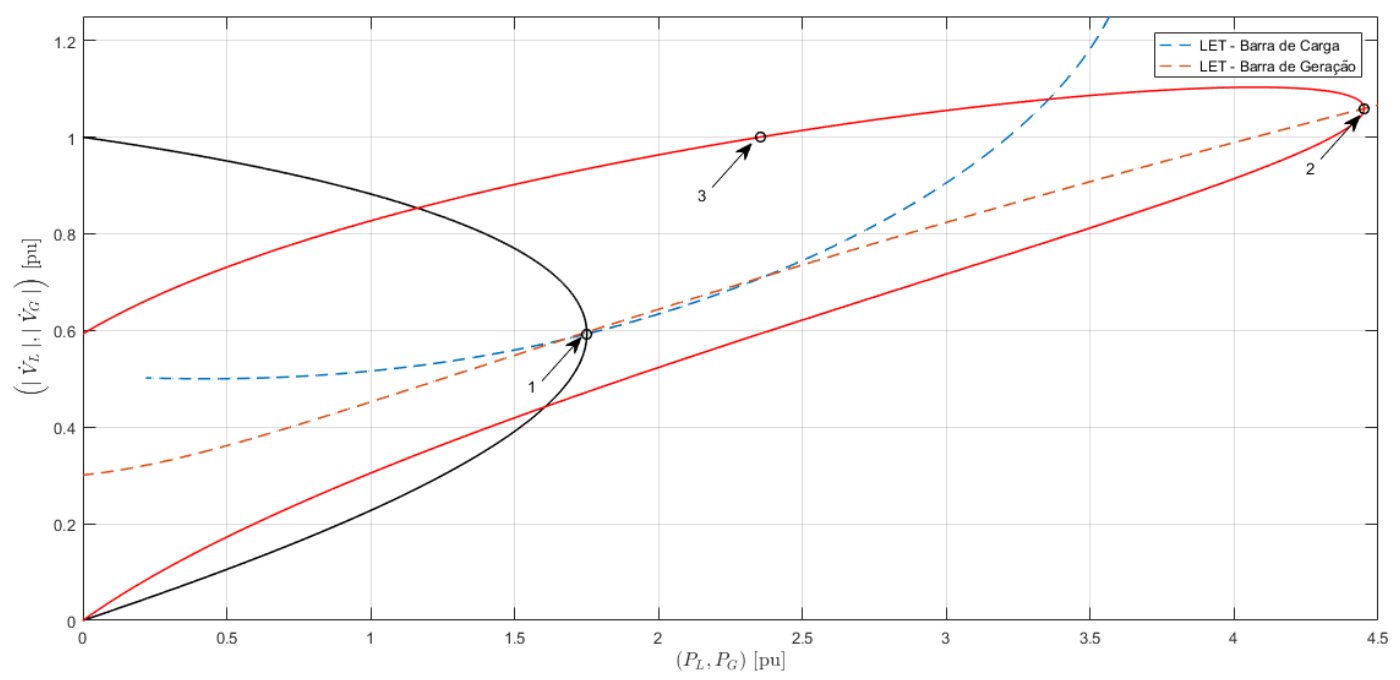

Figura 3.4: Caso 1 - Máxima carga limitada pelo fluxo de potência máximo que chega à barra de carga

Tabela 3.1: Pontos de operação do caso 1

\begin{tabular}{|cccc|}
\hline \multicolumn{4}{|c|}{ Caso 1} \\
\hline \hline \multicolumn{4}{|c|}{ Ponto 1} \\
$\phi\left[^{\circ}\right]$ & $P_{L}^{c}[\mathrm{pu}]$ & $\left|\dot{V}_{L}\right|^{c}[\mathrm{pu}]$ & $\theta_{L G}^{c}\left[^{\circ}\right]$ \\
5,00 & 1,7506 & 0,5928 & $-32,50$ \\
\hline \hline \multicolumn{4}{|c|}{ Ponto 2} \\
$\phi_{G}\left[^{\circ}\right]$ & $P_{G}^{c}[\mathrm{pu}]$ & $\left|\dot{V}_{G}\right|^{c}[\mathrm{pu}]$ & $\theta_{G L}^{c}\left[^{\circ}\right]$ \\
217,50 & 4,4511 & 1,0593 & 73,75 \\
\hline \hline \multicolumn{4}{c}{ Ponto 3} \\
$\phi_{G}\left[^{\circ}\right]$ & $P_{G}[\mathrm{pu}]$ & $\left|\dot{V}_{G}\right|[\mathrm{pu}]$ & $\theta_{G L}\left[^{\circ}\right]$ \\
217,50 & 2,3517 & 1,0000 & 32,50 \\
\hline
\end{tabular}

Caso 2 - Carga com $\phi=-50,00^{\circ}$

No caso 2 , adota-se o ângulo do fator de potência na carga $\phi=-50^{\circ}$, que implica em uma tensão crítica $\dot{V}_{L}^{c}=1,0000 \angle-60^{\circ}[\mathrm{pu}]$ e potência ativa máxima $P_{L}^{\text {máx }}=3,2139[\mathrm{pu}]$, conforme mostrados na Figura 3.5 e na Tabela 3.2 pelo ponto de operação "1". A barra de geração opera no ponto "3" que é coincidente ao ponto " 2 " e, portanto, encontra-se em um cenário de máxima geração. Devese concluir então, que a máxima potência injetada pela barra de geração e a máxima potência entregue à barra de carga atingem simultaneamente o seus máximos, limitando a carga que pode ser alimentada. 


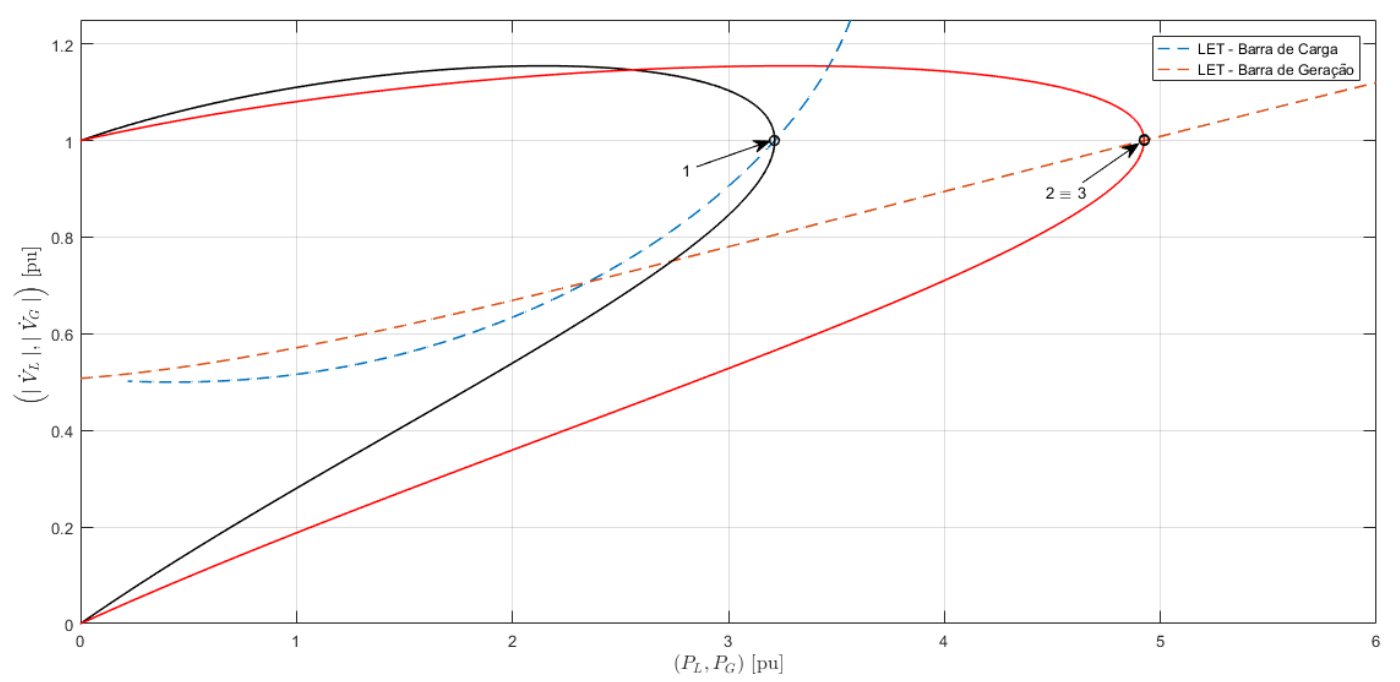

Figura 3.5: Caso 2 - Máxima carga limitada simultaneamente pelo fluxo de potência máximo injetado pela barra terminal do gerador, e pelo fluxo que chega à barra de carga

Tabela 3.2: Pontos de operação do caso 2

\begin{tabular}{|cccc|}
\hline \multicolumn{4}{|c|}{ Caso 2} \\
\hline \hline \multicolumn{4}{|c|}{ Ponto 1} \\
$\phi\left[^{\circ}\right]$ & $P_{L}^{c}[\mathrm{pu}]$ & $\left|\dot{V}_{L}\right|^{c}[\mathrm{pu}]$ & $\theta_{L G}^{c}\left[^{\circ}\right]$ \\
$-50,00$ & 3,2139 & 1,0000 & $-60,00$ \\
\hline \hline \multicolumn{4}{|c}{ Ponto $2 \equiv$ Ponto 3} \\
$\phi_{G}\left[^{\circ}\right]$ & $P_{G}^{c}[\mathrm{pu}]$ & $\left|\dot{V}_{G}\right|^{c}[\mathrm{pu}]$ & $\theta_{G L}^{c}\left[^{\circ}\right]$ \\
190,00 & 4,9240 & 1,0000 & 60,00 \\
\hline
\end{tabular}

Caso 3 - Carga com $\phi=-70,00^{\circ}$

Para o caso 3, toma-se o ponto de operação "1" localizado na região superior da curva $\phi$ constante no plano $P \times|\dot{V}|$ com a tensão na barra de carga $\dot{V}_{L}=1,5315 /-39,93^{\circ}[\mathrm{pu}]$ e a potência ativa consumida $P_{L}=2,6159[\mathrm{pu}] \mathrm{e}$, portanto, ainda distante da condição de máxima carga, denotado na Figura 3.6 e na Tabela 3.3 pelo ponto de operação "2" em que $\dot{V}_{L}^{c}=1,4619 \angle-70^{\circ}$ [pu] e $P_{L}^{\text {máx }}=3,6548[\mathrm{pu}]$. No entanto, para o ponto de operação "2" o gerador atende as solicitações de carga no ponto "3", ou seja, na condição de máxima injeção. O que torna possível concluir que a máxima carga é limitada pelo fluxo de potência que deixa a barra de terminal do gerador. 


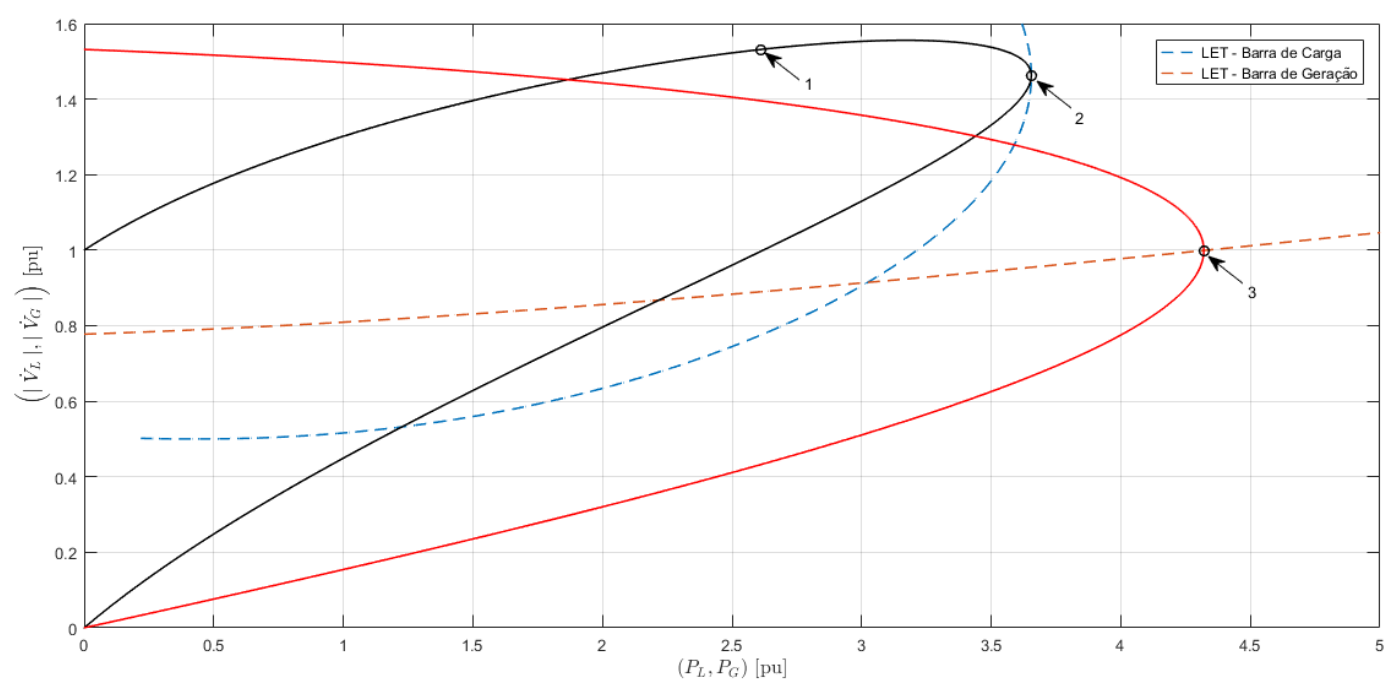

Figura 3.6: Caso 3 - Máxima carga limitada pelo fluxo de potência máximo injetado pela barra terminal do gerador

Tabela 3.3: Pontos de operação do caso 3

\begin{tabular}{|cccc|}
\hline \multicolumn{4}{|c|}{ Caso 3} \\
\hline \hline \multicolumn{4}{|c|}{ Ponto 1} \\
$\phi\left[^{\circ}\right]$ & $P_{L}[\mathrm{pu}]$ & $\left|\dot{V}_{L}\right|[\mathrm{pu}]$ & $\theta_{L G}\left[^{\circ}\right]$ \\
$-70,00$ & 2,6159 & 1,5315 & $-39,93$ \\
\hline \hline \multicolumn{4}{|c|}{ Ponto 2} \\
$\phi\left[^{\circ}\right]$ & $P_{L}^{c}[\mathrm{pu}]$ & $\left|\dot{V}_{L}\right|^{c}[\mathrm{pu}]$ & $\theta_{L G}^{c}\left[^{\circ}\right]$ \\
$-70,00$ & 3,6548 & 1,4619 & $-70,00$ \\
\hline \hline \multicolumn{5}{|c}{ Ponto 3} \\
$\phi_{G}\left[^{\circ}\right]$ & $P_{G}[\mathrm{pu}]$ & $\left|\dot{V}_{G}\right|[\mathrm{pu}]$ & $\theta_{G L}^{c}\left[^{\circ}\right]$ \\
149,94 & 4,3203 & 1,0000 & 39,93 \\
\hline
\end{tabular}

Com base nos resultados apresentados foi possível observar que no sistema de duas barras a ocorrência simultânea de máxima carga e geração está ligada à condição em que o módulo das defasagens angulares críticas são idênticas, ou seja:

$$
\left|\theta_{L G}^{c}\right|=\left|\theta_{G L}^{c}\right|
$$

substituindo (2.20) e (3.15) em (3.17):

$$
\left|\frac{\phi-\alpha_{t}}{2}\right|=\left|\frac{\phi_{G}-\alpha_{t}}{2}\right|
$$

logo, a expressão modular fornece a solução $\phi_{G}=\phi$, trivial e inaceitável sob o aspecto físico, pois $\phi_{G}$ deve ser necessariamente diferente de $\phi$ para contemplar 
as perdas inerentes à transmissão, e a solução factível dada por:

$$
-\left(\frac{\phi-\alpha_{t}}{2}\right)=\frac{\phi_{G}-\alpha_{t}}{2} \Rightarrow \phi_{G}=2 \alpha_{t}-\phi
$$

\section{4}

\section{Conclusões}

Neste capítulo mostrou-se que a existência da máxima carga a ser atendida pelo sistema enfrenta os seguintes fatores limitantes:

- O fluxo de potência que chega à barra de carga.

- O fluxo de potência injetado através da barra terminal do gerador.

- O fluxo de potência que deixa a barra de geração e chega à barra de carga, simultaneamente.

Deste modo, se reforça a necessidade do monitoramento da margem de injeção de potência na rede para a correta identificação a respeito da manifestação da instabilidade de tensão. 


\section{4}

\section{Avaliação das Condições de Estabilidade de Tensão}

A avaliação da estabilidade de tensão em um dado ponto de operação visa lidar com questões associadas à proximidade ao ponto de máximo carregamento, a identificação de áreas consideradas críticas, os principais fatores que contribuem com a instabilidade, e quais ações trazem maior benefício às margens do sistema. Portanto, torna-se de grande importância a utilização de índices que possam traduzir o estado atual da rede.

Devido a sua vasta utilização na literatura, os índices apresentados na próxima seção, baseados no método da matriz $\boldsymbol{D}^{\prime}[15]$ e da matriz reduzida $\boldsymbol{J}_{R}^{Q}[16]$, serão empregados para consolidar os valores numéricos utilizando os equivalentes de Thévenin, e servirão para auxiliar as análises e a localização da barra a ser monitorada.

\section{1}

\section{Método da Matriz $D^{\prime}$}

Apresentado em [15], os índices derivados da matriz $\boldsymbol{D}^{\prime}$ permitem uma avaliação robusta do sistema, sendo capazes de extrair, com base no ponto de operação analisado, informações com significado físico para todas as barras do sistema. Inclusive, sua análise estende-se às barras de tensão controlada, que são de extrema importância sob o aspecto de segurança de tensão, pois são nestas barras em que as ações de controle podem produzir efeito oposto ao esperado. Sejam então as equações linearizadas de fluxo de carga para o sistema, dadas por:

$$
\left[\begin{array}{c}
\Delta \underline{P} \\
\Delta \underline{Q}
\end{array}\right]=\left[\begin{array}{cc}
\frac{\partial \underline{P}}{\partial \theta} & \frac{\partial \underline{P}}{\partial|\dot{\underline{V}}|} \\
\frac{\partial \underline{Q}}{\partial \underline{\theta}} & \frac{\partial \underline{Q}}{\partial|\underline{\dot{V}}|}
\end{array}\right]\left[\begin{array}{c}
\Delta \underline{\theta} \\
\Delta \underline{\dot{\dot{V}} \mid}
\end{array}\right] \Rightarrow\left[\begin{array}{c}
\Delta \underline{P} \\
\Delta \underline{Q}
\end{array}\right]=\underbrace{\left[\begin{array}{cc}
\boldsymbol{J}_{P \theta} & \boldsymbol{J}_{P V} \\
\boldsymbol{J}_{Q \theta} & \boldsymbol{J}_{Q V}
\end{array}\right]}_{\boldsymbol{J}}\left[\begin{array}{c}
\Delta \underline{\theta} \\
\Delta|\dot{V}|
\end{array}\right]
$$

A equação (4.1) pode ser reescrita, deslocando-se para as linhas inferiores as equações relacionadas à barra $i$ em análise:

$$
\left[\begin{array}{l}
\Delta \underline{P}^{\prime} \\
\Delta \underline{Q^{\prime}} \\
\Delta P_{i} \\
\Delta Q_{i}
\end{array}\right]=\left[\begin{array}{ll}
\boldsymbol{A} & \boldsymbol{B} \\
\boldsymbol{C} & \boldsymbol{D}
\end{array}\right]\left[\begin{array}{c}
\Delta \underline{\theta} \\
\Delta|\dot{V}| \\
\Delta \theta_{i} \\
\Delta\left|\dot{V}_{i}\right|
\end{array}\right]
$$


onde as sub-matrizes $\boldsymbol{A}, \boldsymbol{B}, \boldsymbol{C}$, e $\boldsymbol{D}$ correspodem a partições da matriz jacobiano $\boldsymbol{J}$. Uma vez que o interesse é analisar o impacto das variações incrementais de carga nas variáveis de estado da barra em análise $i$, assume-se $\Delta \underline{P}^{\prime}=\underline{0}$ e $\Delta Q^{\prime}=\underline{0}$, de modo que a equação (4.2) torna-se:

$$
\left[\begin{array}{c}
\underline{0} \\
\underline{0} \\
\Delta P_{i} \\
\Delta Q_{i}
\end{array}\right]=\left[\begin{array}{cc}
\boldsymbol{A} & \boldsymbol{B} \\
\boldsymbol{C} & \boldsymbol{D}
\end{array}\right]\left[\begin{array}{c}
\Delta \underline{\theta} \\
\Delta|\dot{V}| \\
\Delta \theta_{i} \\
\Delta\left|\dot{V}_{i}\right|
\end{array}\right]
$$

Desenvolvendo (4.3):

$$
\left[\begin{array}{l}
\underline{0} \\
\underline{0}
\end{array}\right]=\left[\begin{array}{ll}
\boldsymbol{A} & \boldsymbol{B}
\end{array}\right]\left[\begin{array}{c}
\Delta \underline{\theta} \\
\Delta|\dot{V}| \\
\Delta \theta_{i} \\
\Delta\left|\dot{V}_{i}\right|
\end{array}\right]=[\boldsymbol{A}]\left[\begin{array}{c}
\Delta \underline{\theta} \\
\Delta|\dot{V}|
\end{array}\right]+[\boldsymbol{B}]\left[\begin{array}{c}
\Delta \theta_{i} \\
\Delta\left|\dot{V}_{i}\right|
\end{array}\right]
$$

$\log \mathrm{O}$

$$
\left[\begin{array}{c}
\Delta \underline{\theta} \\
\Delta|\dot{V}|
\end{array}\right]=\left[-\boldsymbol{A}^{-1} \boldsymbol{B}\right]\left[\begin{array}{c}
\Delta \theta_{i} \\
\Delta\left|\dot{V}_{i}\right|
\end{array}\right]
$$

Ao mesmo tempo:

$$
\left[\begin{array}{c}
\Delta P_{i} \\
\Delta Q_{i}
\end{array}\right]=\left[\begin{array}{ll}
\boldsymbol{C} & \boldsymbol{D}
\end{array}\right]\left[\begin{array}{c}
\Delta \underline{\theta} \\
\Delta|\dot{V}| \\
\Delta \theta_{i} \\
\Delta\left|\dot{V}_{i}\right|
\end{array}\right]=[\boldsymbol{C}]\left[\begin{array}{c}
\Delta \underline{\theta} \\
\Delta|\dot{V}|
\end{array}\right]+[\boldsymbol{D}]\left[\begin{array}{c}
\Delta \theta_{i} \\
\Delta\left|\dot{V}_{i}\right|
\end{array}\right]
$$

Substituindo (4.4) em (4.5):

$$
\left[\begin{array}{c}
\Delta P_{i} \\
\Delta Q_{i}
\end{array}\right]=\left[\boldsymbol{D}-\boldsymbol{C} \boldsymbol{A}^{-1} \boldsymbol{B}\right]\left[\begin{array}{c}
\Delta \theta_{i} \\
\Delta\left|\dot{V}_{i}\right|
\end{array}\right]
$$

ou:

$$
\left[\begin{array}{c}
\Delta P_{i} \\
\Delta Q_{i}
\end{array}\right]=\left[\boldsymbol{D}^{\prime}\right]\left[\begin{array}{c}
\Delta \theta_{i} \\
\Delta\left|\dot{V}_{i}\right|
\end{array}\right]
$$

Deste modo, obtém-se a sensibilidade entre a injeção de potência na barra $i$ em relação ao módulo e ângulo de tensão, considerando o restante do sistema. Além disso, como será demonstrado, a matriz $\boldsymbol{D}^{\prime}$, de dimensão $2 \times 2$, fornece valiosas informações, pois o sinal e módulo de seu determinante está associado, respectivamente, à região de operação e à distância entre o ponto de operação e o ponto de máximo carregamento. 


\subsection{1}

\section{Módulo do Determinante de $D^{\prime}$}

Considerando inicialmente um sistema de duas barras mostrado na Figura 4.1, pode-se estabelecer a seguinte equação associada ao fluxo de carga:

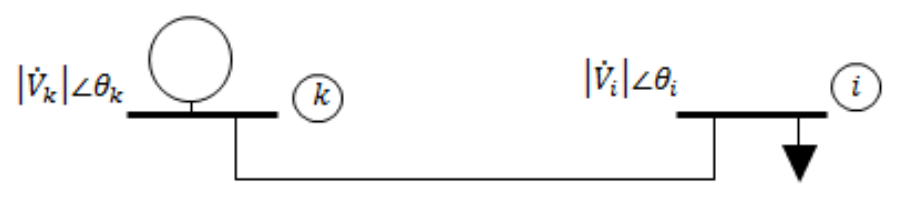

Figura 4.1: Sistema 2 barras

$$
\begin{gathered}
P_{i}=\left|\dot{V}_{i}\right|^{2} G_{i i}+\left|\dot{V}_{i}\right|\left|\dot{V}_{k}\right|\left(G_{i k} \cos \theta_{i k}+B_{i k} \sin \theta_{i k}\right) \\
Q_{i}=-\left|\dot{V}_{i}\right|^{2} B_{i i}+\left|\dot{V}_{i}\right|\left|\dot{V}_{k}\right|\left(G_{i k} \sin \theta_{i k}-B_{i k} \cos \theta_{i k}\right)
\end{gathered}
$$

em que $G_{i k}$ e $B_{i k}$ representam os elementos da matriz de admitância de barra Y.

Nestas circunstâncias, a matriz $\boldsymbol{D}^{\prime}=\boldsymbol{D}=\boldsymbol{J}$, e portanto:

$$
\operatorname{det}\left(\boldsymbol{D}^{\prime}\right)=\frac{\partial P_{i}}{\partial \theta_{i}} \cdot \frac{\partial Q_{i}}{\partial\left|\dot{V}_{i}\right|}-\frac{\partial P_{i}}{\partial\left|\dot{V}_{i}\right|} \cdot \frac{\partial Q_{i}}{\partial \theta_{i}}
$$

onde:

$$
\begin{gathered}
\frac{\partial P_{i}}{\partial \theta_{i}}=\left|\dot{V}_{i}\right|\left|\dot{V}_{k}\right|\left(-G_{i k} \sin \theta_{i k}+B_{i k} \cos \theta_{i k}\right) \\
\frac{\partial P_{i}}{\partial\left|\dot{V}_{i}\right|}=2\left|\dot{V}_{i}\right| G_{i i}+\left|\dot{V}_{k}\right|\left(G_{i k} \cos \theta_{i k}+B_{i k} \sin \theta_{i k}\right) \\
\frac{\partial Q_{i}}{\partial \theta_{i}}=\left|\dot{V}_{i}\right|\left|\dot{V}_{k}\right|\left(G_{i k} \cos \theta_{i k}+B_{i k} \sin \theta_{i k}\right) \\
\frac{\partial Q_{i}}{\partial\left|\dot{V}_{i}\right|}=-2\left|\dot{V}_{i}\right| B_{i i}+\left|\dot{V}_{k}\right|\left(G_{i k} \sin \theta_{i k}-B_{i k} \cos \theta_{i k}\right)
\end{gathered}
$$

Substituindo (4.10),(4.11),(4.12) e (4.13) em (4.9):

$$
\begin{aligned}
& \operatorname{det}\left(\boldsymbol{D}^{\prime}\right)=-\left|\dot{V}_{i}\right|\left|\dot{V}_{k}\right|^{2}\left(G_{i k}^{2}+B_{i k}^{2}\right)+2\left|\dot{V}_{i}\right|^{2}\left|\dot{V}_{k}\right| B_{i i}\left(G_{i k} \sin \theta_{i k}-\right. \\
& \left.B_{i k} \cos \theta_{i k}\right)-2\left|\dot{V}_{i}\right|^{2}\left|\dot{V}_{k}\right| G_{i i}\left(G_{i k} \cos \theta_{i k}+B_{i k} \sin \theta_{i k}\right)
\end{aligned}
$$

Multiplicando (4.14) por $\left|\dot{V}_{i}\right|$, e substituindo $G_{i k}^{2}+B_{i k}^{2}$ por $Y_{i k}^{2}$ :

$\operatorname{det}\left(\boldsymbol{D}^{\prime}\right)\left|\dot{V}_{i}\right|=-\left|\dot{V}_{i}\right|^{2}\left|\dot{V}_{k}\right|^{2} Y_{i k}^{2}+2\left|\dot{V}_{i}\right|^{3}\left|\dot{V}_{k}\right| B_{i i}\left(G_{i k} \sin \theta_{i k}-B_{i k} \cos \theta_{i k}\right)-$ $2\left|\dot{V}_{i}\right|^{3}\left|\dot{V}_{k}\right| G_{i i}\left(G_{i k} \cos \theta_{i k}+\mid B_{i k} \sin \theta_{i k}\right)$

Por outro lado, a potência aparente injetada na barra em análise $\dot{S}_{i}=$ $P_{i}+\mathrm{j} Q_{i}$ pode ser escrita como: 


$$
\begin{aligned}
& \left|\dot{S}_{i}\right|^{2}=P_{i}^{2}+Q_{i}^{2}=\left|\dot{V}_{i}\right|^{4} Y_{i i}^{2}+\left|\dot{V}_{i}\right|^{2}\left|\dot{V}_{k}\right|^{2} Y_{i k}+2\left|\dot{V}_{i}\right|^{3}\left|\dot{V}_{k}\right| G_{i i}\left(G_{i k} \cos \theta_{i k}+\right. \\
& \left.B_{i k} \sin \theta_{i k}\right)-2\left|\dot{V}_{i}\right|^{3}\left|\dot{V}_{k}\right| B_{i i}\left(G_{i k} \sin \theta_{i k}-B_{i k} \cos \theta_{i k}\right)
\end{aligned}
$$

então, substituindo $\left|\dot{S}_{i o}\right|^{2}=\left|\dot{V}_{i}\right|^{4} Y_{i i}^{2}$, tem-se, ao comparar (4.15) e (4.16):

$$
\operatorname{det}\left(\boldsymbol{D}^{\prime}\right)\left|\dot{V}_{i}\right|=\left|\dot{S}_{i o}\right|^{2}-\left|\dot{S}_{i}\right|^{2}
$$

A equação (4.17) permite concluir que a máxima potência aparente ao quadrado a ser injetada na barra em análise, definida pelo termo $\left|\dot{S}_{i o}\right|^{2}$, é função do elemento da diagonal da matriz de admitância de barra e do módulo da tensão na barra $i$. Além disso, no máximo carregamento:

$$
\operatorname{det}\left(\boldsymbol{D}^{\prime}\right)\left|\dot{V}_{i}\right|=0 \Rightarrow\left|\dot{S}_{i o}\right|^{2}-\left|\dot{S}_{i}\right|^{2}=0 \therefore\left|\dot{S}_{i}\right|^{2}=\left|\dot{S}_{i o}\right|^{2}
$$

Deste modo, para o sistema de duas barras:

- $\left|\dot{S}_{i}\right|^{2}$ representa o quadrado da injeção de potência aparente na barra em análise $i$, no ponto de operação corrente

- $\left|\dot{S}_{i o}\right|^{2}$ representa o quadrado da estimativa da máxima injeção de potência aparente, calculado no ponto de operação corrente

$-\operatorname{det}\left(\boldsymbol{D}^{\prime}\right)\left|\dot{V}_{i}\right|$ representa o indicador de distância entre $\left|\dot{S}_{i}\right|^{2}$ e $\left|\dot{S}_{i o}\right|^{2}$

Generalizando o resultado anterior ao sistema multi-nó, pode-se assumir a matriz $\boldsymbol{D}$ como:

$$
\boldsymbol{D}=\left[\begin{array}{ll}
\frac{\partial P_{i}}{\partial \theta_{i}} & \frac{\partial P_{i}}{\partial\left|\dot{V}_{i}\right|} \\
\frac{\partial Q}{\partial \theta_{i}} & \frac{\partial Q_{i}}{\partial\left|\dot{V}_{i}\right|}
\end{array}\right]=\left[\begin{array}{ll}
x & z \\
y & u
\end{array}\right]
$$

ainda, nomeando os elementos da matriz obtida por:

$$
\left[-\boldsymbol{C A}^{-1} \boldsymbol{B}\right]=\left[\begin{array}{ll}
b_{1} & b_{3} \\
b_{2} & b_{4}
\end{array}\right]
$$

ao desenvolver a igualdade $\boldsymbol{D}^{\prime}=\left[\boldsymbol{D}-\boldsymbol{C A}^{-1} \boldsymbol{B}\right]$, chega-se a:

$$
\begin{gathered}
\operatorname{det}\left(\boldsymbol{D}^{\prime}\right)=\operatorname{det}\left(\left[\boldsymbol{D}-\boldsymbol{C} \boldsymbol{A}^{-1} \boldsymbol{B}\right]\right) \therefore \\
\operatorname{det}\left(\boldsymbol{D}^{\prime}\right)=\operatorname{det}\left(\left[\begin{array}{ll}
x & z \\
y & u
\end{array}\right]-\left[\begin{array}{ll}
b_{1} & b_{3} \\
b_{2} & b_{4}
\end{array}\right]\right) \therefore \\
\operatorname{det}\left(\boldsymbol{D}^{\prime}\right)=\underbrace{[x \cdot u-y \cdot z]}_{\operatorname{det}(D)}+\underbrace{\left[x \cdot b_{4}-y \cdot b_{3}\right]+\left[b_{1} \cdot u-b_{2} \cdot z\right]+\left[b_{1} \cdot b_{4}-b_{2} \cdot b_{3}\right]}_{\Delta \operatorname{det}}
\end{gathered}
$$


portanto, multiplicando ambos os lados da igualdade por $\left|\dot{V}_{i}\right|$, obtém-se a forma generalizada ao sistema multi-nó:

$$
\operatorname{det}\left(\boldsymbol{D}^{\prime}\right)\left|\dot{V}_{i}\right|=\operatorname{det}(\boldsymbol{D})\left|\dot{V}_{i}\right|+\Delta \operatorname{det}\left|\dot{V}_{i}\right|
$$

ou:

$$
\operatorname{det}\left(\boldsymbol{D}^{\prime}\right)\left|\dot{V}_{i}\right|=\left|\dot{S}_{i o}\right|^{2}-\Delta \operatorname{det}\left|\dot{V}_{i}\right|-\left|\dot{S}_{i}\right|^{2}
$$

onde:

- $\left|\dot{S}_{i}\right|^{2}$ representa o quadrado da injeção de potência aparente na barra em análise $i$, no ponto de operação corrente

- $\left|\dot{S}_{i o}\right|^{2}$ representa o quadrado da estimativa da máxima injeção de potência aparente, calculado no ponto de operação corrente

- $\Delta$ det $\left|\dot{V}_{i}\right|$ representa a influência do restante do sistema, que limita a injeção de potência na barra $i$

- $S_{m}^{2}=\left|\dot{S}_{i o}\right|^{2}-\Delta$ det $\left|\dot{V}_{i}\right|$ representa o quadrado da estimativa da máxima potência aparente, considerando o restante do sistema

$-\operatorname{det}\left(\boldsymbol{D}^{\prime}\right)\left|\dot{V}_{i}\right|$ representa o indicador de distância entre $S_{m}^{2}$ e $\left|\dot{S}_{i o}\right|^{2}$

\subsection{2}

\section{Sinal de $\operatorname{det}\left(\boldsymbol{D}^{\prime}\right)$}

A Figura 4.2 mostra as curvas $P_{i}, Q_{i}$ e $\phi_{i}$ constantes no plano $\theta \times|\dot{V}|$, onde $P_{i}$ e $Q_{i}$ representam as potências ativa e reativa transmitidas à barra de carga $i$, e $\phi_{i}$ representa o ângulo do fator de potência da carga. Conforme descrito anteriormente, para uma potência transmitida diferente da máxima, verifica-se a existência de duas soluções de tensão para atender a carga: o ponto $A$ que pertence à parte superior da curva $S \times|\dot{V}|$, sendo conhecido como solução de alta tensão e baixa corrente, e o ponto $B$, que pertence à parte inferior da curva $S \times|\dot{V}|$, conhecido como solução de baixa tensão e alta corrente. Em particular, no ponto de máximo carregamento, representado pelo ponto $C$, a solução de tensão é única.

Representando os vetores gradiente $\nabla \vec{P}_{i}$ e $\nabla \vec{Q}_{i}$ na base ortonormal, obtém-se a expressão:

$$
\begin{aligned}
& \nabla \vec{P}_{i}=\frac{\partial P_{i}}{\partial \theta_{i}} \vec{i}+\frac{\partial P_{i}}{\partial\left|\dot{V}_{i}\right|} \vec{j}+0 \vec{k} \\
& \nabla \vec{Q}_{i}=\frac{\partial Q_{i}}{\partial \theta_{i}} \vec{i}+\frac{\partial Q_{i}}{\partial\left|\dot{V}_{i}\right|} \vec{j}+0 \vec{k}
\end{aligned}
$$




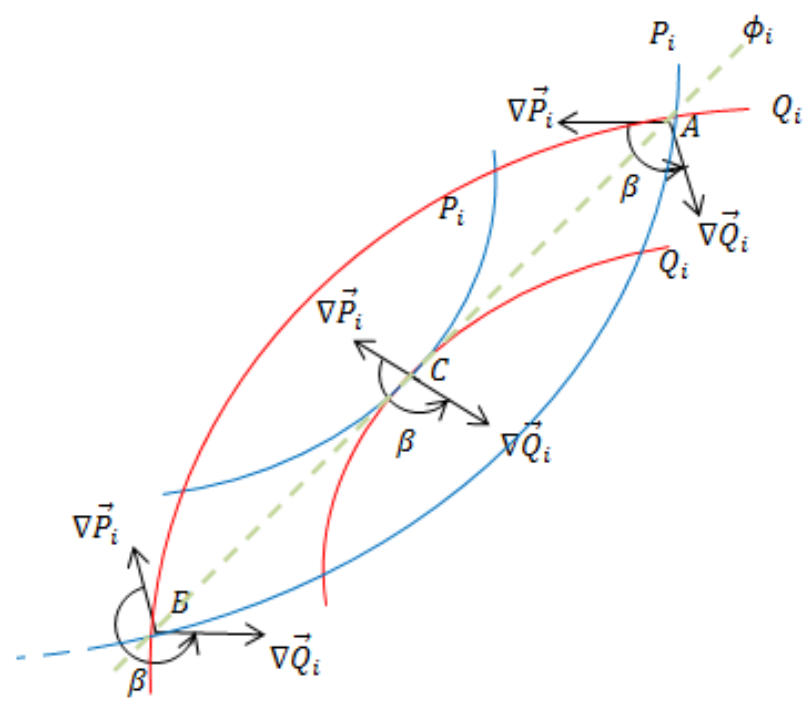

Figura 4.2: Localização do vetor gradiente $\nabla \vec{P}_{i}$ e $\nabla \vec{Q}_{i}$ no plano $\theta \times|\dot{V}|$

O produto vetorial entre $\nabla \vec{P}_{i}$ e $\nabla \vec{Q}_{i}$ é dado por:

$$
\nabla \vec{P}_{i} \times \nabla \vec{Q}_{i}=\left[\left(\frac{\partial P_{i}}{\partial \theta_{i}} \cdot \frac{\partial Q_{i}}{\partial\left|\dot{V}_{i}\right|}\right)-\left(\frac{\partial P_{i}}{\partial\left|\dot{V}_{i}\right|} \cdot \frac{\partial Q_{i}}{\partial \theta_{i}}\right)\right] \vec{k}
$$

ou seja:

$$
\nabla \vec{P}_{i} \times \nabla \vec{Q}_{i}=\operatorname{det}\left(\boldsymbol{D}^{\prime}\right) \vec{k}
$$

Sabendo que (4.25) também pode ser reescrito como:

$$
\nabla \vec{P}_{i} \times \nabla \vec{Q}_{i}=\left[\left|\nabla \vec{P}_{i}\right|\left|\nabla \vec{Q}_{i}\right| \sin \beta\right] \vec{k}
$$

onde $\beta$ representa o ângulo entre os vetores gradiente $\nabla \vec{P}_{i}$ e $\nabla \vec{Q}_{i}$. Então, igualando (4.26) e (4.27):

$$
\operatorname{det}\left(\boldsymbol{D}^{\prime}\right)=\left|\nabla \vec{P}_{i}\right|\left|\nabla \vec{Q}_{i}\right| \sin \beta
$$

de onde se conclui que:

$$
\begin{aligned}
& -\operatorname{det}\left(\boldsymbol{D}^{\prime}\right)>0 \quad \Leftrightarrow \quad \sin \beta>0 \text {, i.e. } 0^{\circ}<\beta<180^{\circ} \\
& -\operatorname{det}\left(\boldsymbol{D}^{\prime}\right)<0 \quad \Leftrightarrow \quad \sin \beta<0 \text {, i.e. }-180^{\circ}<\beta<0^{\circ} \\
& -\operatorname{det}\left(\boldsymbol{D}^{\prime}\right)=0 \quad \Leftrightarrow \quad \sin \beta=0 \text {, i.e. } \beta=k 180^{\circ} \quad(k=-1,0,1)
\end{aligned}
$$

Tomando $\nabla \vec{P}_{i}$ como referência, pode-se então observar que na região normal de operação $A$, o ângulo $\beta$ é tal que $0^{\circ}<\beta<180^{\circ}$ e o determinante $\operatorname{det}\left(\boldsymbol{D}^{\prime}\right)>0$. Na parte inferior da curva $|\dot{S}| \times|\dot{V}|$, correspondente à região anormal de operação $B,-180^{\circ}<\beta<0^{\circ}$ e, portanto, o determinante $\operatorname{det}\left(\boldsymbol{D}^{\prime}\right)<0$. Por sua vez, no ponto $C$, os vetores gradientes estão alinhados, e o ângulo $\beta$ será $\beta= \pm 180^{\circ}$ ou $\beta=0^{\circ}$, oque implica em $\operatorname{det}\left(\boldsymbol{D}^{\prime}\right)=0$.

Nota-se portanto, que $\beta$ pode ser utilizado de modo complementar, 
como indicativo da distância angular entre o ponto de operação em análise e o máximo carregamento. Dito de outro modo, o ângulo $\beta$ representa a dificuldade do sistema em admitir a injeção de potência, sendo máximo quando a defasagem aproxima-se de $180^{\circ}$ [15].

\subsection{3}

\section{Interpretação dos Índices}

Importante ressaltar que para todos os índices demonstrados, sua validade é instantânea, tendo em vista que o método emprega dados referentes ao ponto de operação corrente. Isto significa que no minuto seguinte, devido à dinâmica inerente ao sistema de potência, seja pelas variações de carga, de geração, compensação de potência reativa, ou pelos LTCs atingindo os limites de tape, os índices podem perder seu sentido, e as inferências, principalmente relativas à margem, devem considerar este aspecto.

O valor $S_{m}-\left|\dot{S}_{i}\right|$ representa a margem em MVA, entre a potência aparente injetada na barra $i$ e a máxima potência aparente a ser injetada, calculado para o ponto de operação em análise. E o sinal do ângulo $\beta$ (ou do $\operatorname{det}\left(\boldsymbol{D}^{\prime}\right)$ ) é o indicador da região de operação.

Deste modo, para o ponto de operação 1 mostrado na Figura 4.3 e localizado na parte superior da curva $\phi$ constante no plano $|\dot{S}| \times|\dot{V}|$, a margem $S_{m}-\left|\dot{S}_{i}\right|$ representa a potência aparente que pode ser injetada na barra $i$, mantendo-se o fator de potência constante, até o máximo carregamento. Para tornar coerente a avaliação, e estabelecer corretamente a ordem de importância das barras, divide-se a margem por $S_{m}$, obtendo a margem normalizada $M$, dada em percentual por:

$$
M_{i}=\left(1-\frac{\left|\dot{S}_{i}\right|}{S_{m}}\right) \times 100 \%
$$

Assim, a barra crítica pode ser diretamente indicada como a barra de menor margem $M$.

Considerando que o ponto de operação encontra-se na parte inferior da curva $\phi$ constante no plano $|\dot{S}| \times|\dot{V}|$, ou seja, no ponto de operação 3 da Figura 4.3, a margem $S_{m}-\left|\dot{S}_{i}\right|$ é traduzida como a potência aparente em MVA que pode ser retirada de $\left|\dot{S}_{i}\right|$ para se alcançar o máximo carregamento. A margem $M$ normalizada, portanto, será sempre negativa e dada neste caso por:

$$
M_{i}=\left(\frac{S_{m}}{\left|\dot{S}_{i}\right|}-1\right) \times 100 \%
$$




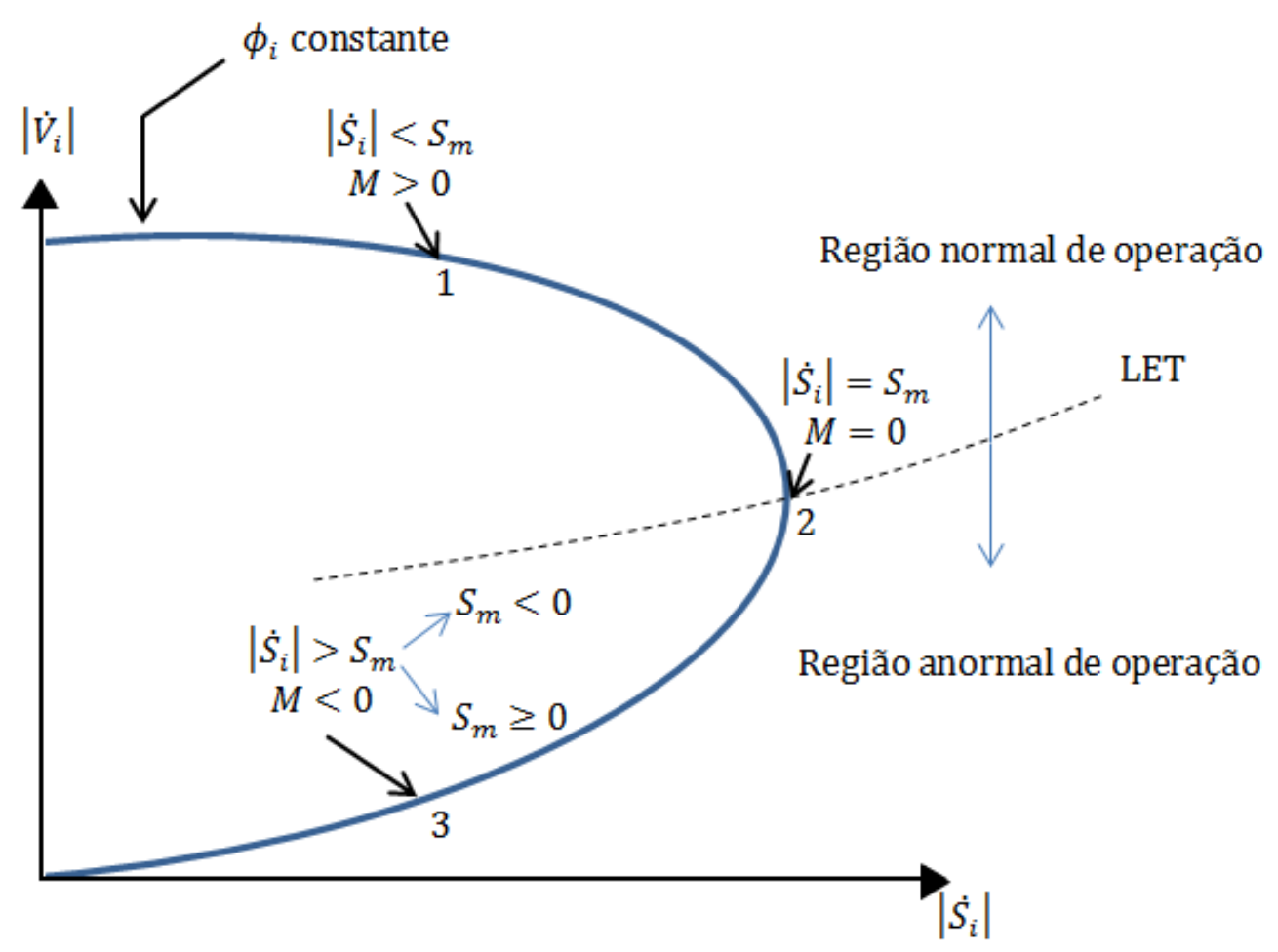

Figura 4.3: Sinal da margem $M$ associado à curva $\phi$ constante em diferentes pontos de operação no plano $|\dot{S}| \times|\dot{V}|$

Para a operação no ponto de máximo carregamento, identificado na Figura 4.3 pelo ponto de operação 2 , a margem $S_{m}-\left|\dot{S}_{i}\right|$ é nula, o que significa que não há o que se adicionar ou retirar de $\left|\dot{S}_{i}\right|$ para atingir $S_{m}$, ou seja, $M=0$. Nas barras de passagem, onde não há injeção líquida de potência, a margem calculada será de 100\%, e com o aumento no carregamento do sistema, a potência $S_{m}$ calculada tenderá a diminuir devido ao aumento do fluxo de potência na barra, e o valor de $\beta$ aumentará até eventualmente corresponder a uma defasagem de $\pm 180^{\circ}$.

\subsection{4}

\section{Avaliação de Barras de Tensão Controlada}

Conforme exposto anteriormente, a robustez do método da matriz $\boldsymbol{D}^{\prime}$ reside em sua capacidade de avaliar barras de tensão controlada, que são de grande importância, pois as ações relativas ao controle de tensão a elas associadas podem produzir o efeito oposto ao esperado. Na prática, a tensão das barras de tensão controlada não são constantes, mas oscilam em torno de um valor especificado na faixa de "banda morta" do equipamento controlador, como pode ser observado na Figura 4.4. Em sua ultrapassagem, as ações de controle corretivo são executadas pelo regulador automático de tensão, que a leva ao valor especificado. 


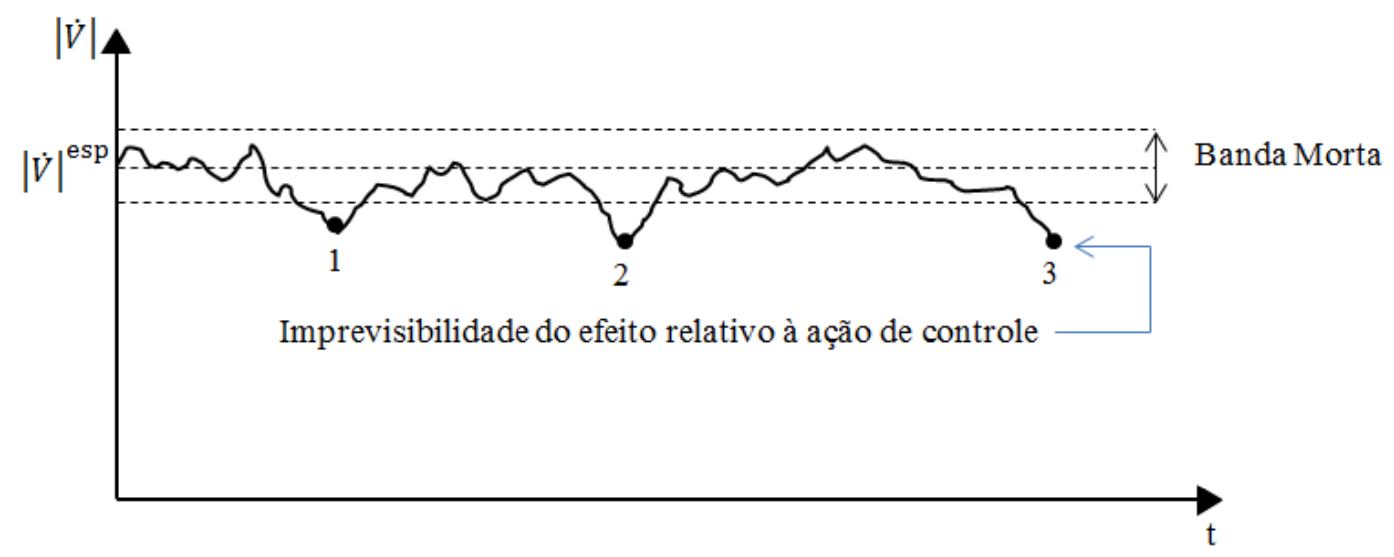

Figura 4.4: Variação no tempo da tensão na barra de tensão controlada

Deste modo, conhecer o ponto de operação relativo à barra em análise é fundamental, pois, sabe-se que dada a localização na parte superior da cuva $\phi$ constante no plano $|\dot{S}| \times|\dot{V}|$, identificado pelos pontos 1 e 2 nas Figuras 4.4 e 4.5 , as ações de controle serão efetivas. No entanto, uma vez que o ponto de operação encontra-se na parte inferior da curva $\phi$ constante no plano $|\dot{S}| \times|\dot{V}|$, como o identificado pelo ponto 3, perde-se a previsibilidade das ações de controle e, portanto, ações corretivas podem deteriorar o sistema, sob o ponto de vista de segurança de tensão.

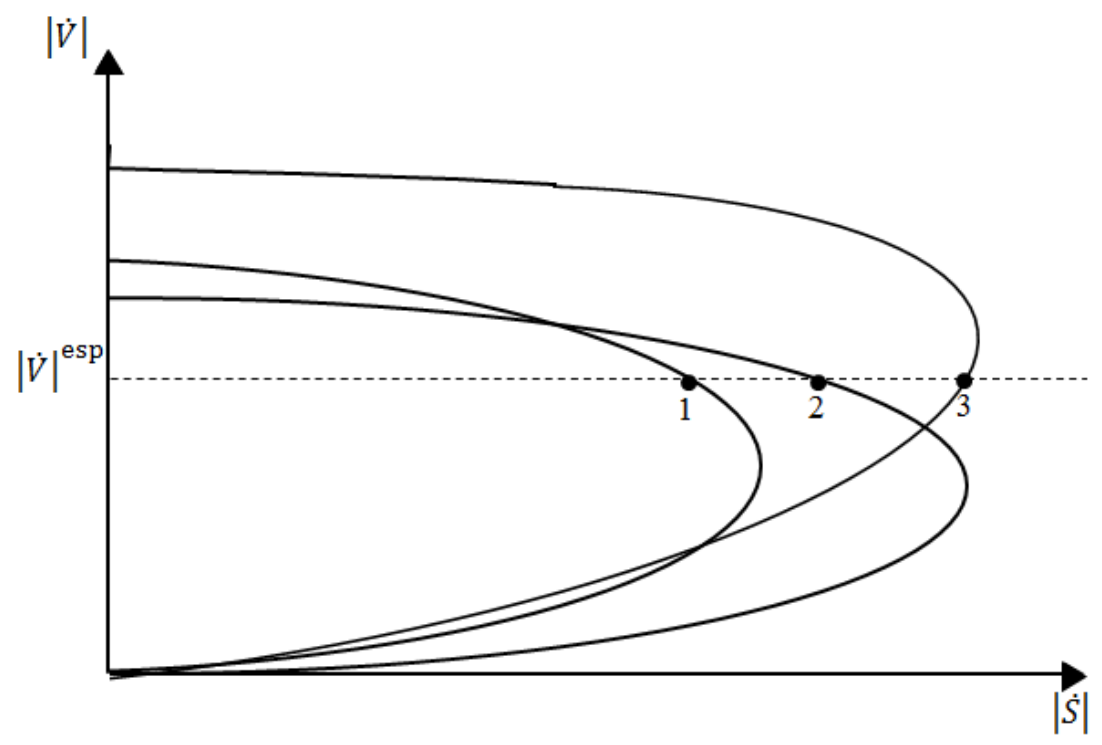

Figura 4.5: Localização dos pontos de operação na curva $\phi$ constante através do plano $|\dot{S}| \times|\dot{V}|$

Para analisar este tipo de barra considera-se uma perda no controle de tensão da barra e, portanto, seu tratamento torna-se equivalente ao de uma 
barra PQ.

\section{2}

\section{Análise por Autovalores e Autovetores}

O método baseado na análise de autovalores e autovetores [16] estabelece como critério para avaliação da estabilidade de tensão a sensibilidade entre o módulo da tensão nas barras do sistema e a injeção de potência reativa. Para tanto, consideram-se apenas as variações incrementais de potência reativa na equação de fluxo de carga em sua forma linearizada apresentada em (4.1). Ou seja:

$$
\left[\begin{array}{c}
\underline{0} \\
\Delta \underline{Q}
\end{array}\right]=\left[\begin{array}{ll}
\boldsymbol{J}_{P \theta} & \boldsymbol{J}_{P V} \\
\boldsymbol{J}_{Q \theta} & \boldsymbol{J}_{Q V}
\end{array}\right]\left[\begin{array}{c}
\Delta \underline{\theta} \\
\Delta|\underline{\dot{V}}|
\end{array}\right]
$$

através de algumas manipulações na expressão (4.31), chega-se a:

$$
\Delta \underline{Q}=\underbrace{\left[\boldsymbol{J}_{Q V}-\boldsymbol{J}_{Q \theta} \boldsymbol{J}_{P \theta}^{-1} \boldsymbol{J}_{P V}\right]}_{\boldsymbol{J}_{R}^{Q}} \Delta|\dot{V}|
$$

portanto:

$$
\Delta|\underline{\dot{V}}|=\boldsymbol{J}_{R}^{Q^{-1}} \Delta \underline{Q}
$$

onde $\boldsymbol{J}_{R}^{Q}$ simboliza a matriz jacobiana reduzida do sistema, e cujos elementos representam a sensibilidade entre as injeções de potência reativa e o módulo da tensão nas barras PQ.

Decompondo a matriz $\boldsymbol{J}_{R}^{Q}$ nas matrizes de autovetores à direita $\boldsymbol{\Xi}$, de autovetores à esquerda $\boldsymbol{\Gamma}$, e da matriz diagonal de autovalores $\boldsymbol{\Lambda}$, obtém-se:

$$
\begin{gathered}
\boldsymbol{\Xi}=\left[\begin{array}{ccccc}
\mid & \mid & \mid & \mid & \mid \\
\xi_{1} & \cdots & \xi_{i} & \cdots & \xi_{n} \\
\mid & \mid & \mid & \mid & \mid
\end{array}\right] \boldsymbol{\Lambda}=\left[\begin{array}{ccccc}
\lambda_{1} & \cdots & 0 & \cdots & 0 \\
\vdots & \ddots & 0 & & 0 \\
0 & \cdots & \lambda_{i} & \cdots & 0 \\
\vdots & & \vdots & \ddots & \vdots \\
0 & \cdots & 0 & \cdots & \lambda_{n}
\end{array}\right] \boldsymbol{\Gamma}=\left[\begin{array}{ccc}
- & \gamma_{1} & - \\
- & \vdots & - \\
- & \gamma_{i} & - \\
- & \vdots & - \\
- & \gamma_{n} & -
\end{array}\right] \\
\boldsymbol{J}_{R}^{Q}=\boldsymbol{\Xi} \boldsymbol{\Lambda} \boldsymbol{\Gamma}
\end{gathered}
$$

donde segue que:

$$
\boldsymbol{J}_{R}^{Q^{-1}}=\boldsymbol{\Xi} \boldsymbol{\Lambda}^{-1} \boldsymbol{\Gamma}
$$

deste modo, introduzindo a identidade (4.34) na equação (4.33):

$$
\Delta|\underline{\dot{V}}|=\boldsymbol{\Xi} \Lambda^{-1} \boldsymbol{\Gamma} \Delta \underline{Q}
$$

ou:

$$
\Delta|\underline{\dot{V}}|=\sum_{i=1}^{n} \frac{\xi_{i} \gamma_{i}}{\lambda_{i}} \Delta \underline{Q}
$$

Cada autovalor $\lambda_{i}$ e seus correspondentes autovetores a direita $\xi_{i}$, e a esquerda e $\gamma_{i}$, definem o $i$-ésimo modo. Uma prática comum é tomar o vetor em sua 
forma normalizada, de tal maneira que:

$$
\Xi \Gamma=I \Rightarrow \Xi^{-1}=\Gamma
$$

Portanto, desde que $\boldsymbol{\Xi}^{-1}=\boldsymbol{\Gamma}$, a equação (4.35) pode ser reescrita como:

$$
\Gamma \Delta|\underline{\dot{V}}|=\Lambda^{-1} \Gamma \Delta \underline{Q}
$$

Ao definir $\boldsymbol{v}=\boldsymbol{\Gamma} \Delta|\underline{\dot{V}}|$ como vetor de variação de tensão modal, e $\boldsymbol{q}=\boldsymbol{\Gamma} \Delta \underline{Q}$ como vetor de variação de potência reativa modal, então a equação (4.37) torna-se:

$$
\boldsymbol{v}=\boldsymbol{\Lambda}^{-1} \boldsymbol{q}
$$

Para o $i$-ésimo modo:

$$
\boldsymbol{v}_{i}=\frac{1}{\lambda_{i}} \boldsymbol{q}_{i}
$$

Como o módulo da tensão modal é igual ao produto do inverso de $\lambda_{i}$ pela variação de potência reativa modal, é possível inferir o grau de estabilidade do sistema com base na magnitude de $\lambda_{i}$. Portanto, sendo $\lambda_{i}>0$, então a variação de tensão modal e a variação de potência reativa modal possuem a mesma direção. Isto implica em um sistema estável, pois em dado o ponto de operação, um aumento na injeção de potência reativa produz um aumento no módulo da tensão das barras.

Em contrapartida, para $\lambda_{i}<0$, a variação de tensão modal e a variação de potência reativa modal possuem direções opostas, indicando que o sistema pode ser instável, dado que no ponto de operação em análise, um aumento na injeção de potência reativa produz, em ao menos uma das barras do sistema, um decréscimo no módulo da tensão.

Quanto mais próximo de zero se encontrar a magnitude de $\lambda_{i}$ positivo, mais a tensão modal se aproxima da instabilidade. Estes modos são denominados críticos. Quando $\lambda_{i}=0$ a tensão modal colapsa, pois para qualquer variação infinitesimal no módulo da tensão, necessita-se de uma injeção infinita de potência reativa.

Em relação às características da matriz Jacobiana reduzida, se $\boldsymbol{J}_{R}^{Q}$ é simétrica, então seus autovalores e autovetores são reais, e além disso, os autovetores à direita e os autovetores à esquerda são iguais. Para fins práticos, a matriz $\boldsymbol{J}_{R}^{Q}$ pode ser tomada como uma matriz aproximadamente simétrica [16].

Assumindo $\Delta \underline{Q}=\underline{e}_{k}$ na equação (4.36), onde $\underline{e}_{k}$ é um vetor nulo, exceto na posição $k$, que possui valor igual a 1 , então:

$$
\Delta|\underline{\dot{V}}|=\sum_{i=1}^{n} \frac{\gamma_{i k} \xi_{i}}{\lambda_{i}}
$$


A sensibilidade $|\dot{V}|$-Q em relação a barra $k$ será dada por:

$$
\frac{\partial|\dot{V}|_{k}}{\partial Q_{k}}=\sum_{i=1}^{n} \frac{\xi_{k i} \gamma_{i k}}{\lambda_{i}}=\sum_{i=1}^{n} \frac{\Phi_{k i}}{\lambda_{i}}
$$

onde $\Phi_{k i}$ é denominado fator de participação da barra $k$ no modo $i$. 


\section{5}

\section{Métodos de Detecção da Instabilidade de Tensão}

Conforme observado no Capítulo 1, com desenvolvimento de unidades de medição fasorial no início da década de 90, associado ao desenvolvimento tecnológico e da infraestrutura das redes de comunicação, criou-se um nova perspectiva acerca do monitoramento do sistema elétrico. Recentemente, a criação de uma revisão sistemática a partir do levantamento bibliográfico realizado através da base de dados Scopus, considerando a utilização de medições fasoriais na avaliação da estabilidade de tensão é apresentada em [17], e tem como objetivo identificar as tendências entre as diversas metodologias. Tais abordagens foram classificadas de acordo com a Tabela 5.1, e algumas conclusões destacadas pelos autores a respeito dos artigos considerados relevantes para o tema indicam que as análises empregando o vasto monitoramento do sistema são superiores àquelas baseadas em medições locais. Ademais, os métodos, em sua maioria, comprometem-se com a detecção da instabilidade de tensão, sendo a utilização do equivalente de rede o que representa, dentro deste conjunto, o maior volume de contribuições.

Uma visão geral sobre os métodos de detecção da instabilidade de tensão, tema central deste trabalho, é apresentado em [18], em que as metodologias são agrupadas em dois grandes blocos: os métodos baseados em medições locais e os métodos baseados em um amplo monitoramento. Neste contexto, pode-se observar que os equivalentes de rede podem ser incluídos em ambos os grupos dependendo da metodologia empregada.

O fenômeno da estabilidade de tensão foi caracterizado no Capítulo 2, através de um sistema simples de duas barras, que pode representar o equivalente de Thévenin "visto" pela barra em análise. De fato, torna-se inconsiste a utilização direta do teorema em sistemas elétricos de potência devido à natureza não-linear associada às equações de fluxo de carga. Contudo, como será visto em detalhes nas seções seguintes, um grande esforço tem sido direcionado ao desenvolvimento de metodologias visando estimar os parâmetros do circuito equivalente e a obtenção de índices. 
Tabela 5.1: Características avaliadas por [17]

\begin{tabular}{|c|c|c|}
\hline Item & Características & Opções \\
\hline $\mathrm{A}$ & Tipos de perturbações & $\begin{array}{l}\text { Pequenas perturbações } \\
\text { Grandes perturbações } \\
\text { Pequenas e grandes perturbações }\end{array}$ \\
\hline B & Simulação & $\begin{array}{l}\text { Longo termo } \\
\text { Curto termo } \\
\text { Longo e curto termo }\end{array}$ \\
\hline $\mathrm{C}$ & Tipo de análise & $\begin{array}{l}\text { Estática } \\
\text { Dinâmica }\end{array}$ \\
\hline $\mathrm{D}$ & Aplicação & $\begin{array}{l}\text { Online } \\
\text { Tempo real }\end{array}$ \\
\hline $\mathrm{E}$ & Monitoramento & $\begin{array}{l}\text { Local } \\
\text { Amplo }\end{array}$ \\
\hline $\mathrm{F}$ & Tipo de avaliação & $\begin{array}{l}\text { Detecção } \\
\text { Predição }\end{array}$ \\
\hline G & Tipos de medições utilizadas & $\begin{array}{l}\text { Pré-distúrbio } \\
\text { Pós-distúrbio } \\
\text { Pré e pós-distúrbio }\end{array}$ \\
\hline $\mathrm{H}$ & Abordagem de controle & $\begin{array}{l}\text { Manual } \\
\text { Automático }\end{array}$ \\
\hline I & Método de análise & $\begin{array}{l}\text { Analítico } \\
\text { Equivalentes de rede } \\
\text { Inteligência artificial }\end{array}$ \\
\hline
\end{tabular}

5.1

Método VIP

O método proposto em [19], retratado neste trabalho como método VIP ("Voltage Instability Predictor"), utiliza o equacionamento do equivalente de Thévenin. Deste modo, segue que:

$$
\dot{E}_{t h}=\dot{V}_{L}+\dot{I}_{L} \bar{Z}_{t h}
$$

em componentes retangulares:

$$
E_{t h_{\text {real }}}+j E_{\text {thimag }}=V_{L_{\text {real }}}+j V_{L_{\text {imag }}}+\left(I_{L_{\text {real }}}+j I_{L_{\text {imag }}}\right)\left(R_{t h}+j X_{t h}\right)
$$

logo, matricialmente obtém-se: 


$$
\left[\begin{array}{cccc}
1 & 0 & -I_{L_{\text {real }}} & I_{L_{\text {imag }}} \\
0 & 1 & -I_{L_{\text {imag }}} & -I_{L_{\text {real }}}
\end{array}\right]\left[\begin{array}{c}
E_{t h_{\text {real }}} \\
E_{t h_{\text {imag }}} \\
R_{t h} \\
X_{t h}
\end{array}\right]=\left[\begin{array}{c}
V_{L_{\text {real }}} \\
V_{L_{\text {imag }}}
\end{array}\right]
$$

Dado que as quatro variáveis referentes ao equivalente de Thévenin são desconhecidas, a resolução do conjunto de equações necessita, portanto, de medições consecutivas nos instantes $t_{i}$ e $t_{i+1}$. Considera-se então que durante tais medições os parâmetros do equivalente de Thévenin permaneçam constantes. Para lidar com as variações devido a medições imprecisas, pode-se utilizar uma janela amostral de maior tamanho de modo a minimizar os erros de estimação dos parâmetros pelo método dos mínimos quadrados.

Em decorrência do método VIP, emprega-se em [20] o monitoramento do sistema através da quantificação da margem ao ponto crítico em um sistema de grande porte. Os esforços despendidos na concepção do método VIP originamse da criação de um projeto de pesquisa conduzido pela AEB ("American Eletric Power") e a ABB visando aplicações reais no sistema elétrico.

\section{2}

\section{Método de Šmon, Verbič e Gubina (ŠVG)}

Em [21], utiliza-se o teorema de Tellegen, cuja aplicação não restringese apenas a sistemas lineares. A demonstração do teorema generalizado é apresentado em [22], onde se observa que a validade do método é estendida a todos os sistema que obedecem às leis de Kirchhoff. Uma das propriedades do Teorema de Tellegen deve-se à sua forma diferencial, utilizada em sistemas com pontos de operação distintos e com a mesma topologia:

$$
\hat{\boldsymbol{I}}^{T} \Delta \boldsymbol{V}-\hat{\boldsymbol{V}}^{T} \Delta \boldsymbol{I}=\boldsymbol{O}
$$

onde $\Delta \boldsymbol{V}$ e $\Delta \boldsymbol{I}$ representam os vetores de variações de tensão e corrente nos ramos e barras da rede incrementada $N$ no caso base, e $\hat{\boldsymbol{V}}$ e $\hat{\boldsymbol{I}}$ representam os vetores de tensão e corrente nos ramos e barras da rede adjunta $\hat{N}$.

Adaptando a equação (5.3) para o sistema elétrico de potência, pode-se definir os seguintes conjuntos:

- Conjunto $s$ definido pela barra swing do sistema;

- Conjunto $p$ definido pelas barras remanescentes;

- Conjunto $r$ de ramos do sistema;

Deste modo, a equação (5.3) pode ser reescrita como:

$$
\left(\hat{\boldsymbol{I}}_{s}^{T} \Delta \boldsymbol{V}_{s}-\hat{\boldsymbol{V}}_{s}^{T} \Delta \boldsymbol{I}_{s}\right)+\left(\hat{\boldsymbol{I}}_{p}^{T} \Delta \boldsymbol{V}_{p}-\hat{\boldsymbol{V}}_{p}^{T} \Delta \boldsymbol{I}_{p}\right)+\left(\hat{\boldsymbol{I}}_{r}^{T} \Delta \boldsymbol{V}_{r}-\hat{\boldsymbol{V}}_{r}^{T} \Delta \boldsymbol{I}_{r}\right)=\boldsymbol{O}
$$


O termo referente à barra swing não possui contribuição em (5.4), pois na rede adjunta a barra swing é modelada como curto-circuito, portanto $\hat{\boldsymbol{V}}_{s}^{T}=\boldsymbol{O}$. Ainda se pode assumir que a barra swing, tal qual uma barra infinita, é invariável em relação à tensão e frequência no modelo da rede $N$, então $\Delta \boldsymbol{V}_{s}=\boldsymbol{O}$. Por sua vez, o termo referente aos ramos também não possui contribuições em (5.4), pois apenas as perturbações na rede afetam esta parcela. Como deseja-se avaliar perturbações de potência, onde as redes $N$ e $\hat{N}$ são topologicamente idênticas, então $\Delta \boldsymbol{Y}=\boldsymbol{O}$. Deste modo, a equação (5.4) torna-se:

$$
\left(\hat{\boldsymbol{I}}_{p}^{T} \Delta \boldsymbol{V}_{p}-\hat{\boldsymbol{V}}_{p}^{T} \Delta \boldsymbol{I}_{p}\right)=\boldsymbol{O}
$$

Em [22], demonstra-se que sendo $\Lambda$ um operador linear denominado operador linear de Kirchhoff, a equação (5.5) pode ser reescrita, sem perda de generalidade, em função desta transformação linear. Definindo como operador linear, o conjugado complexo aplicado ao vetor de corrente, então:

$$
\left(\hat{\boldsymbol{I}}_{p}^{* T} \Delta \boldsymbol{V}_{p}-\hat{\boldsymbol{V}}_{p}^{T} \Delta \boldsymbol{I}_{p}^{*}\right)=\boldsymbol{O}
$$

Admitindo que ao analisar a barra $j$, o restante do sistema possa ser substituído pelo equivalente de Thévenin e sua rede adjunta, conforme mostrado na Figura 5.1, então a equação (5.6) pode ser utilizada através de medições fasoriais consecutivas na barra em análise.

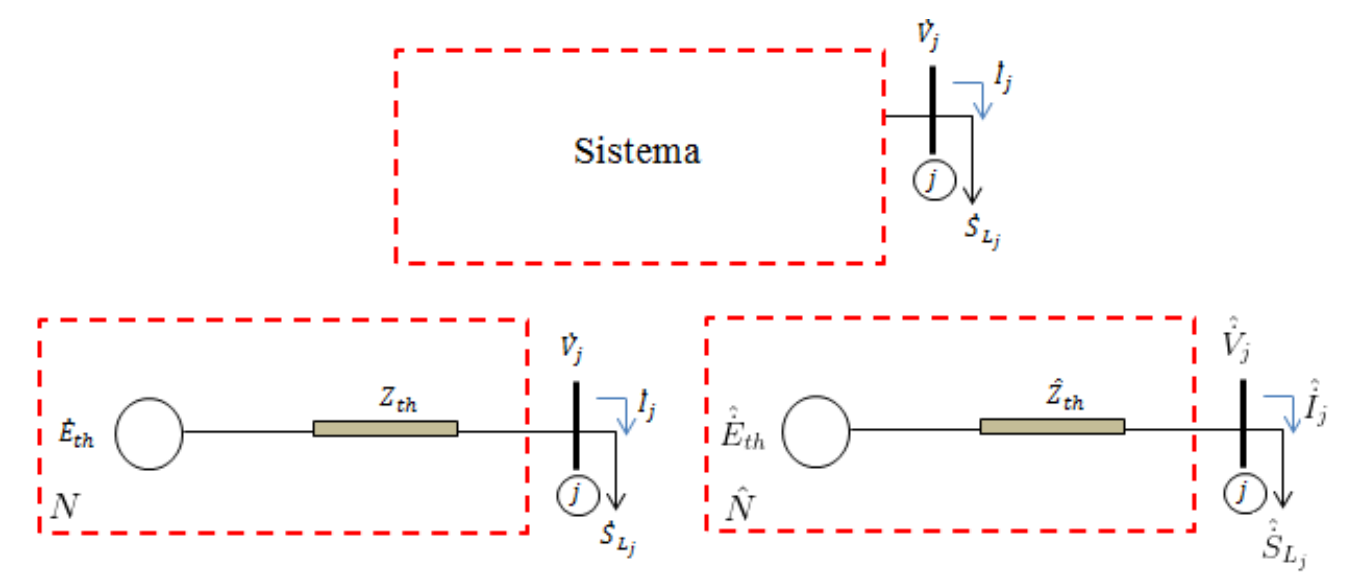

Figura 5.1: Barra em análise $j$ do sistema, a rede de Thévenin correspondente $N$, e sua rede adjunta $\hat{N}$

Deste modo:

$$
\hat{\dot{I}}_{j}^{*} \Delta \dot{V}_{j}-\hat{\dot{V}}_{j} \Delta \dot{I}_{j}^{*}=0
$$

onde $\Delta \dot{V}_{j}$ e $\Delta \dot{I}_{j}^{*}$ representam as variações de tensão e corrente na barra $j$ em relação aos valores do caso base $\dot{V}_{j}$ e $\dot{I}_{j}^{*}$, obtidos a partir da perturbação da rede $N$. Por sua vez, $\hat{\dot{V}}_{j}$ e $\hat{\dot{I}}_{j}^{*}$ representam os fasores de tensão e corrente na barra $j$ da rede adjunta $\hat{N}$.

Tendo em vista que a corrente drenada pela barra $j$ é dada por: 


$$
\hat{\dot{I}}_{j}^{*}=\frac{\hat{\dot{S}}_{j}}{\hat{\dot{V}}_{j}}=\left(\frac{\hat{\dot{E}}_{t h}-\hat{\dot{V}}_{j}}{\hat{Z}_{t h}}\right)^{*}
$$

então, substituindo (5.8) em (5.7):

$$
\left(\frac{\hat{\dot{E}}_{t h}-\hat{\dot{V}}_{j}}{\hat{Z}_{t h}}\right)^{*} \Delta \dot{V}_{j}-\hat{\dot{V}}_{j} \Delta \dot{I}_{j}^{*}=0
$$

$\log \mathrm{O}$

$$
\hat{Z}_{t h}^{*}=\frac{\left(\hat{\dot{E}}_{t h}-\hat{\dot{V}}_{j}\right)^{*} \Delta \dot{V}_{j}}{\hat{\dot{V}}_{j} \Delta \dot{I}_{j}^{*}}
$$

Ao considerar que na máxima transferência de potência no circuito equivalente de Thévenin, a queda de tensão na impedância $\hat{Z}_{t h}$ é igual à tensão na carga $\hat{\dot{V}}_{j}$, a equação (5.10) reduz-se a:

$$
\hat{Z}_{t h}^{*}=\frac{\Delta \dot{V}_{j}}{\Delta \dot{I}_{j}^{*}} \Rightarrow \hat{Z}_{t h}=\frac{\Delta \dot{V}_{j}^{*}}{\Delta \dot{I}_{j}}
$$

Para contornar inconsistências de ordem numérica, define-se uma tolerância $|\Delta \dot{I}|^{\text {mín }}$ para o qual $\left|\Delta \dot{I}_{j}\right|<|\Delta \dot{I}|^{\text {mín }}$, a impedância na medição atual mantém-se idêntica ao valor anterior.

Em condições normais de operação $\left|\hat{Z}_{t h}\right|<<\left|\hat{Z}_{L_{j}}\right|$, mas no ponto de máximo carregamento $\left|\hat{Z}_{t h}\right|=\left|\hat{Z}_{L_{j}}\right|$, portanto:

$$
\left|\frac{\Delta \dot{V}_{j}}{\Delta \dot{I}_{j}}\right|=\left|\frac{\dot{V}_{j}}{\dot{I}_{j}}\right|
$$

O índice de estabilidade de impedância normalizada (ISI) é apresentado e definido em [21] por:

$$
\mathrm{ISI}=1-\left|\frac{\hat{Z}_{t h}}{\hat{Z}_{L_{j}}}\right|
$$

e que compreende o intervalo de zero a 1 , sendo zero o indicativo de máximo carregamento.

\section{3}

\section{Método de Corsi-Taranto}

O algoritmo adaptativo apresentado em [23, 24] foi desenvolvido para barras de EAT dotadas de cargas indutivas. Deste modo, no equivalente "visto" pela barra em análise, a reatância é consideravelmente maior que a resistência equivalente e, portanto, $X_{t h}>R_{t h}$. É razoável admitir que $R_{t h} \approx 0$, e sob estas circunstâncias o conjunto de equações pode ser substancialmente simplificado. O diagrama fasorial do circuito de Thévenin é mostrado na Figura 5.2 . 


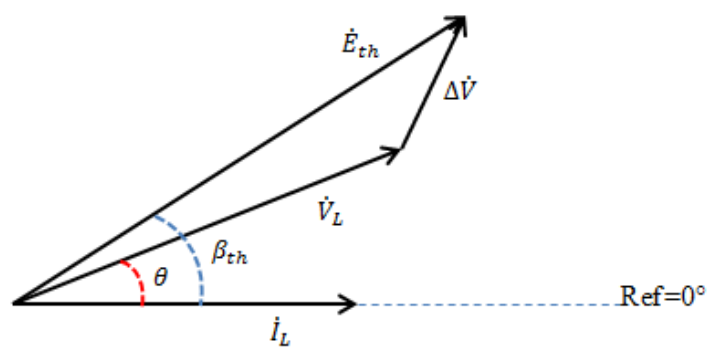

Figura 5.2: Diagrama fasorial do equivalente de Thévenin

De acordo com o diagrama, obtém-se expressão:

$$
\begin{gathered}
\dot{E}_{t h}=\dot{V}_{L}+\Delta \dot{V} \\
\dot{E}_{t h}=\dot{V}_{L}+\dot{I}_{L} \bar{Z}_{t h}
\end{gathered}
$$

decompondo (5.14) nas componentes real e imaginária:

$$
\begin{aligned}
& \left|\dot{E}_{t h}\right| \cos \left(\beta_{t h}\right)=\left|\dot{V}_{L}\right| \cos (\theta)+\left|\dot{I}_{L}\right| R_{t h} \\
& \left|\dot{E}_{t h}\right| \sin \left(\beta_{t h}\right)=\left|\dot{V}_{L}\right| \sin (\theta)+\left|\dot{I}_{L}\right| X_{t h}
\end{aligned}
$$

tomando $R_{t h}=0$, é possível isolar $\beta_{t h}$ em (5.15), onde:

$$
\beta_{t h}^{0}=\cos ^{-1}\left(\frac{\left|\dot{V}_{L}\right| \cos (\theta)}{\left|\dot{E}_{t h}\right|^{0}}\right)
$$

Os parâmetros $\left|\dot{V}_{L}\right|$ e $\theta$ são obtidos por medição fasorial na barra em análise. Então, a estimativa inicial para $\beta_{t h}^{0}$ depende apenas de $\left|\dot{E}_{t h}\right|^{0}$, e se torna necessário, portanto, conhecer a faixa de valores de $\left|\dot{E}_{t h}\right|$. Uma boa estimativa inicial para $\left|\dot{E}_{t h}\right|^{0}$ é tomar sua média aritmética:

$$
\left|\dot{E}_{t h}\right|^{0}=\frac{\left|\dot{E}_{t h}\right|^{\text {mín }}+\left|\dot{E}_{t h}\right|^{\text {máx }}}{2}
$$

onde:

$$
\begin{gathered}
\left|\dot{E}_{t h}\right|^{\text {mín }}=\left|\dot{V}_{L}\right| \\
\left|\dot{E}_{t h}\right|^{\text {máx }}=\left|\dot{V}_{L}\right| \sqrt{2(1+\sin \theta)}
\end{gathered}
$$

A reatância $X_{t h}^{0}$ pode ser calculada por (5.16), donde segue que:

$$
X_{t h}^{0}=\frac{\left|\dot{E}_{t h}\right|^{0} \sin \left(\beta_{t h}^{0}\right)-\left|\dot{V}_{L}\right| \sin (\theta)}{\left|\dot{I}_{L}\right|}
$$

Durante o processo, a variável $\left|\dot{E}_{t h}\right|$ é atualizada, e conforme demonstrase em [23], é possível tomar uma direção de atualização de $\left|\dot{E}_{t h}\right|$ a fim de se produzir maior velocidade na convergência de $X_{t h}$. 
Para isto, supõe-se que os parâmetros $\left|\dot{E}_{t h}\right|$ e $X_{t h}$ permaneçam constantes durante as medições consecutivas $i-1$ e $i$, suficientemente próximas. Define-se então que, ao se identificar o valor exato da tensão de Thévenin $\dot{E}_{t h}$ através da variável estimada $\dot{\widetilde{E}}_{t h}$, comete-se um erro de estimação $\widetilde{\Delta} \dot{E}_{t h}$. Deste modo:

$$
\underbrace{\dot{\widetilde{E}}_{t h}}_{\text {valor estimado }}=\underbrace{\dot{E}_{t h}}_{\text {valor real }}+\underbrace{\widetilde{\Delta} \dot{E}_{t h}}_{\text {erro }}
$$

Analogamente, define-se que ao se identificar o valor exato da reatância de Thévenin $X_{t h}$, através da variável estimada $\widetilde{X}_{t h}$, comete-se o erro de estimação, dado por $\widetilde{\Delta} X_{t h}$. Então:

$$
\underbrace{j \widetilde{X}_{t h}}_{\text {valor estimado }}=\underbrace{j X_{t h}}_{\text {valor real }}+\underbrace{j \widetilde{\Delta} X_{t h}}_{\text {erro }}
$$

Substituindo as equações (5.22) e (5.23) em (5.14), obtém-se:

$$
\dot{E}_{t h}+\widetilde{\Delta} \dot{E}_{t h}=\dot{V}_{L}+\dot{I}_{L}\left(j X_{t h}+j \widetilde{\Delta} X_{t h}\right)
$$

onde $\dot{I}_{L}=\left|\dot{I}_{L}\right| \angle 0^{\circ}$, e $\dot{E}_{t h}=\dot{V}_{L}+\dot{I}_{L} j X_{t h}$. Portanto:

$$
\dot{E}_{t h}+\widetilde{\Delta} \dot{E}_{t h}=\dot{E}_{t h}+\left|\dot{I}_{L}\right| j \widetilde{\Delta} X_{t h}
$$

$\log \mathrm{O}$

$$
\widetilde{\Delta} \dot{E}_{t h}=j\left|\dot{I}_{L}\right| \widetilde{\Delta} X_{t h}=j \widetilde{\Delta}\left|\dot{E}_{t h}\right|
$$

De acordo com a equação (5.26) é possível observar que o erro de identificação associados às variáveis $\left|\dot{E}_{t h}\right|$ e $X_{t h}$ possuem a mesma direção. Considerando a variação de carga nos instantes $i-1$ e $i$, e mantendo $\widetilde{\Delta}\left|\dot{E}_{t h}\right|$ constante, se estabelecem as seguintes relações:

$$
\begin{gathered}
j \widetilde{\Delta}\left|\dot{E}_{t h}\right|=j \widetilde{\Delta} X_{t h}^{i-1}\left|\dot{I}_{L}\right|^{i-1} \Rightarrow j \widetilde{\Delta} X_{t h}^{i-1}=\frac{j \widetilde{\Delta}\left|\dot{E}_{t h}\right|}{\left|I_{L}\right|^{i-1}} \\
j \widetilde{\Delta}\left|\dot{E}_{t h}\right|=j \widetilde{\Delta} X_{t h}^{i}\left|\dot{I}_{L}\right|^{i} \Rightarrow j \widetilde{\Delta} X_{t h}^{i}=\frac{j \widetilde{\Delta}\left|\dot{E}_{t h}\right|}{\left|I_{L}\right|^{i}}
\end{gathered}
$$

subtraindo (5.27) de (5.28):

$$
j \widetilde{\Delta} X_{t h}^{i}-j \widetilde{\Delta} X_{t h}^{i-1}=j \widetilde{\Delta}\left|\dot{E}_{t h}\right| \times\left(\frac{\left|\dot{I}_{L}\right|^{i-1}-\left|\dot{I}_{L}\right|^{i}}{\left|\dot{I}_{L}\right|^{i-1} \times\left|\dot{I}_{L}\right|^{i}}\right)
$$

como o erro de estimação representa a diferença entre o valor estimado e o valor real, mostrado na equação (5.23), então:

$$
\begin{gathered}
\left(j \widetilde{X}_{t h}^{i}-j X_{t h}\right)-\left(j \widetilde{X}_{t h}^{i-1}-j X_{t h}\right)=j \widetilde{\Delta}\left|\dot{E}_{t h}\right| \times\left(\frac{\left|\dot{I}_{L}\right|^{i-1}-\left|\dot{I}_{L}\right|^{i}}{\left|\dot{I}_{L}\right|^{i-1} \times\left|\dot{I}_{L}\right|^{i}}\right) \\
j\left(\widetilde{X}_{t h}^{i}-\widetilde{X}_{t h}^{i-1}\right)=j \widetilde{\Delta}\left|\dot{E}_{t h}\right| \times\left(\frac{\left|\dot{I}_{L}\right|^{i-1}-\left|\dot{I}_{L}\right|^{i}}{\left|\dot{I}_{L}\right|^{i-1} \times\left|\dot{I}_{L}\right|^{i}}\right)
\end{gathered}
$$

Portanto, ao supor um cenário definido por um aumento de carga, ou 
seja, $\Delta\left|Z_{L}\right|<0$, com eventual superestimação de $\left|\dot{E}_{t h}\right|$, se segue para uma superestimação de $j X_{t h}$, de acordo com a equação (5.30), pois:

$$
\begin{aligned}
& -\left|\dot{I}_{L}\right|^{i-1}-\left|\dot{I}_{L}\right|^{i}<0 \\
& -\widetilde{\Delta}\left|\dot{E}_{t h}\right|>0 \\
& -j\left(\widetilde{X}_{t h}^{i}-\widetilde{X}_{t h}^{i-1}\right)<0
\end{aligned}
$$

Deste modo, sendo $j\left(\widetilde{X}_{t h}^{i}-\widetilde{X}_{t h}^{i-1}\right)<0$, então $j \widetilde{X}_{t h}^{i}<j \widetilde{X}_{t h}^{i-1}$. Ou seja, o valor estimado de $\left|\dot{E}_{t h}\right|$ deve tomar uma direção no sentido de ser reduzido em relação à estimativa anterior.

Por outro lado, em uma eventual subestimação de $\left|\dot{E}_{t h}\right|$ :

$$
\begin{aligned}
& -\left|\dot{I}_{L}\right|^{i-1}-\left|\dot{I}_{L}\right|^{i}<0 \\
& -\widetilde{\Delta}\left|\dot{E}_{t h}\right|<0 \\
& -j\left(\widetilde{X}_{t h}^{i}-\widetilde{X}_{t h}^{i-1}\right)>0
\end{aligned}
$$

Portanto, $j\left(\widetilde{X}_{t h}^{i}-\widetilde{X}_{t h}^{i-1}\right)>0$, então $j \widetilde{X}_{t h}^{i}>\widetilde{X}_{t h}^{i-1}$. Ou seja, o valor estimado de $\left|\dot{E}_{t h}\right|$ deve tomar uma direção no sentido de ser incrementado em relação à estimativa anterior.

De modo análogo, é possível estabelecer as direções de atualização das variáveis $\left|\dot{E}_{t h}\right|$ e $X_{t h}$ para $\Delta\left|Z_{L}\right|<0$. Em particular, quando $\Delta\left|Z_{L}\right|=0$ não é possível definir a direção de atualização e o valor de $\left|\dot{E}_{t h}\right|$ permanece inalterado.

Conhecida a faixa permitida para a variável $\left|\dot{E}_{t h}\right|$, e sua direção de atualização, o método então consiste em deixá-la variar livremente dentro dos limites, e estipular um parâmetro para o controle de atualização. Isto é feito, tomando:

$$
\epsilon_{E}=\operatorname{mín}\left\{\epsilon_{\text {inf }}, \epsilon_{\mathrm{sup}}, \epsilon_{\mathrm{lim}}\right\}
$$

onde:

$$
\begin{gathered}
\epsilon_{\text {inf }}=\left|\dot{E}_{t h}^{i-1}-\dot{V}_{L}^{i}\right| \\
\epsilon_{\text {sup }}=\left|\dot{E}_{t h}^{i-1}-\dot{E}_{t h}^{\operatorname{máx}(i)}\right| \\
\epsilon_{\text {lim }}=\left|\dot{E}_{t h}^{i-1} \times k\right|
\end{gathered}
$$

O parâmetro que majora a atualização de $\left|\dot{E}_{t h}\right|$ durante as simulações é $\epsilon_{\lim }$ e, portanto, a escolha do parâmetro pré-especificado $k$ deve ser adequada de modo que permita um equilíbrio entre a velocidade de atualização e a precisão buscada [24].

O algoritmo do método adaptativo é sintetizado da seguinte forma: 


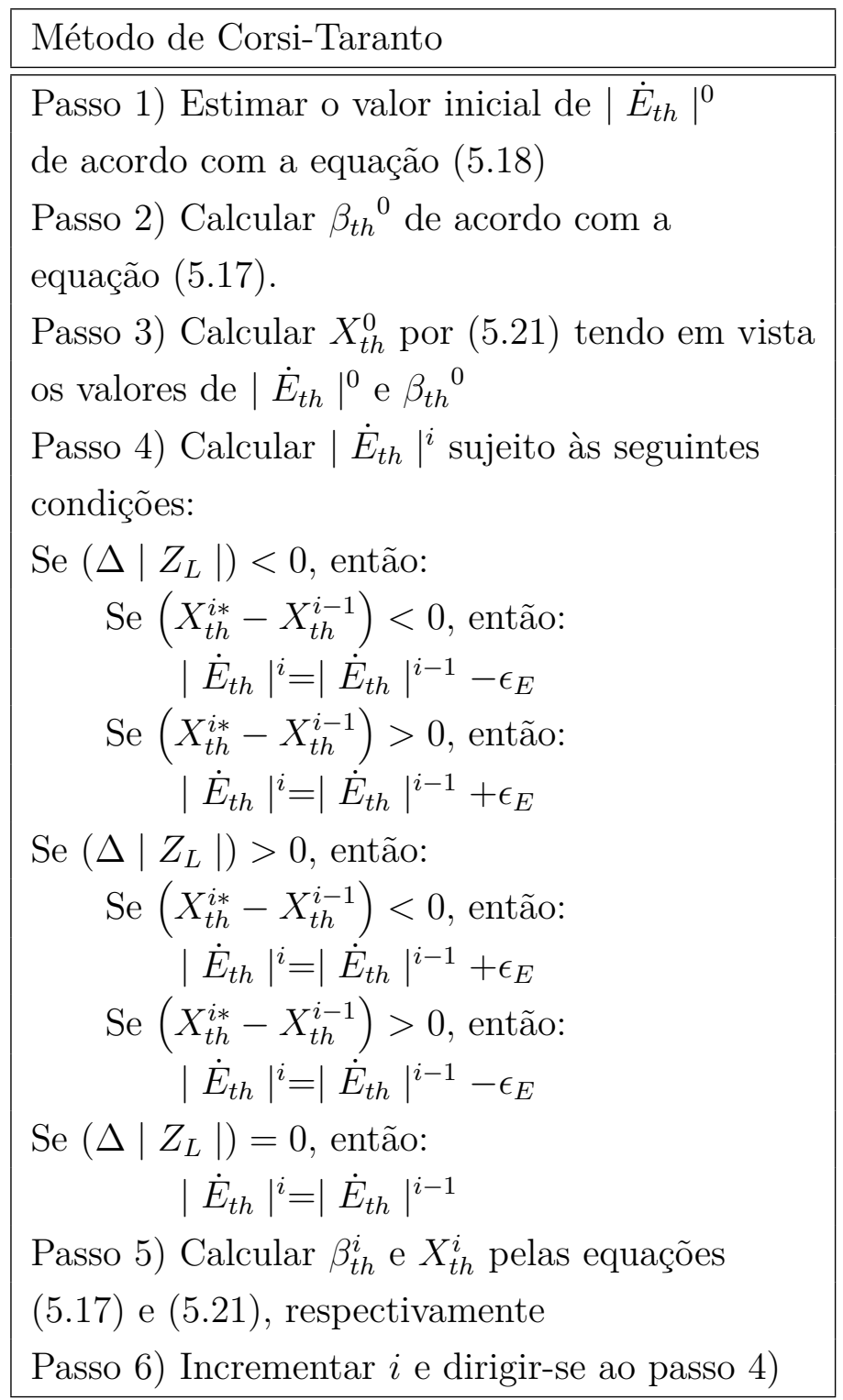

Importante destacar que $X_{t h}^{i *}$ representa o valor intermediário de $X_{t h}^{i}$ tendo em vista o valores atualizados de tensão e corrente, e os valores anteriores $\left|\dot{E}_{t h}\right|$ e $\beta_{t h}$.

\section{4}

\section{Circuito Acoplado de Porta Simples (CAPS)}

O método baseado em circuitos acoplados de portas simples [25] apresenta uma formulação do equivalente de Thévenin multi-portas. Segundo este modelo, a rede multi-portas pode ser construída extraindo-se do sistema o conjunto de cargas e de geradores, como na Figura 5.3. 


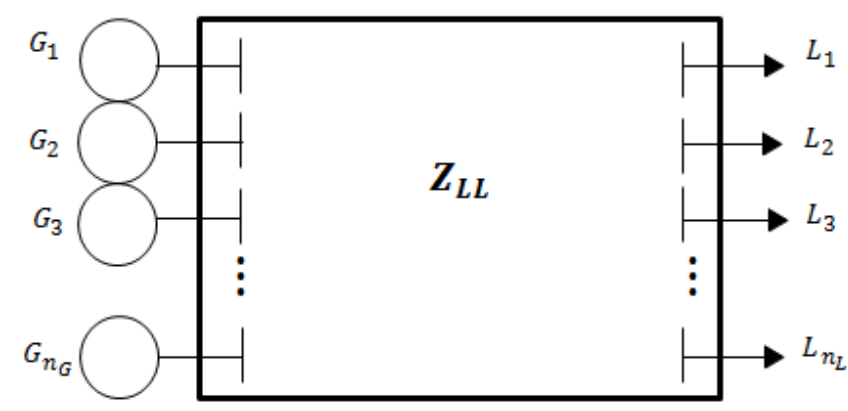

Figura 5.3: Modelo da rede multi-portas

O sistema pode então ser descrito por:

$$
\left[\begin{array}{c}
-\boldsymbol{I}_{L} \\
\mathbf{0} \\
\boldsymbol{I}_{G}
\end{array}\right]=\left[\begin{array}{ccc}
\boldsymbol{Y}_{L L} & \boldsymbol{Y}_{L T} & \boldsymbol{Y}_{L G} \\
\boldsymbol{Y}_{T L} & \boldsymbol{Y}_{T T} & \boldsymbol{Y}_{T G} \\
\boldsymbol{Y}_{G L} & \boldsymbol{Y}_{G T} & \boldsymbol{Y}_{G G}
\end{array}\right]\left[\begin{array}{c}
\boldsymbol{V}_{L} \\
\boldsymbol{V}_{T} \\
\boldsymbol{V}_{G}
\end{array}\right]
$$

Os subíndices $L, T$ e $G$ representam, respectivamente, as barras de carga, de passagem e de geração. Reescrevendo a equação (5.35):

$$
\begin{gathered}
-\boldsymbol{I}_{L}=\boldsymbol{Y}_{L L} \boldsymbol{V}_{L}+\boldsymbol{Y}_{L T} \boldsymbol{V}_{T}+\boldsymbol{Y}_{L G} \boldsymbol{V}_{G} \\
\boldsymbol{O}=\boldsymbol{Y}_{T L} \boldsymbol{V}_{L}+\boldsymbol{Y}_{T T} \boldsymbol{V}_{T}+\boldsymbol{Y}_{T G} \boldsymbol{V}_{G} \\
\boldsymbol{I}_{G}=\boldsymbol{Y}_{G L} \boldsymbol{V}_{L}+\boldsymbol{Y}_{G T} \boldsymbol{V}_{T}+\boldsymbol{Y}_{G G} \boldsymbol{V}_{G}
\end{gathered}
$$

isolando o termo $\boldsymbol{Y}_{T L} \boldsymbol{V}_{L}$ em (5.36):

$$
\boldsymbol{Y}_{L L} \boldsymbol{V}_{L}=-\boldsymbol{Y}_{L T} \boldsymbol{V}_{T}-\boldsymbol{Y}_{L G} \boldsymbol{V}_{G}-\boldsymbol{I}_{L}
$$

segue de (5.37), isolando a tensão no conjunto de barras de passagem $\boldsymbol{V}_{T}$ :

$$
\boldsymbol{V}_{T}=\boldsymbol{Y}_{T T}^{-1}\left(-\boldsymbol{Y}_{Z L} \boldsymbol{V}_{L}-\boldsymbol{Y}_{T G} \boldsymbol{V}_{G}\right)
$$

inserindo a identidade obtida em (5.40) em (5.39), chega-se a:

$$
\boldsymbol{Y}_{L L} \boldsymbol{V}_{L}=-\boldsymbol{Y}_{L T} \boldsymbol{Y}_{Z Z}^{-1}\left(-\boldsymbol{Y}_{T L} \boldsymbol{V}_{L}-\boldsymbol{Y}_{T G} \boldsymbol{V}_{G}\right)-\boldsymbol{Y}_{L G} \boldsymbol{V}_{G}-\boldsymbol{I}_{L}
$$

$$
\boldsymbol{Y}_{L L} \boldsymbol{V}_{L}=\boldsymbol{Y}_{L T} \boldsymbol{Y}_{T T}^{-1} \boldsymbol{Y}_{T L} \boldsymbol{V}_{L}+\boldsymbol{Y}_{L T} \boldsymbol{Y}_{T T}^{-1} \boldsymbol{Y}_{T G} \boldsymbol{V}_{G}-\boldsymbol{Y}_{L G} \boldsymbol{V}_{G}-\boldsymbol{I}_{L}
$$

rearranjando a expressão (5.41):

$$
\left(\boldsymbol{Y}_{L L}-\boldsymbol{Y}_{L T} \boldsymbol{Y}_{T T}^{-1} \boldsymbol{Y}_{T L}\right) \boldsymbol{V}_{L}=\left(\boldsymbol{Y}_{L T} \boldsymbol{Y}_{T T}^{-1} \boldsymbol{Y}_{T G}-\boldsymbol{Y}_{L G}\right) \boldsymbol{V}_{G}-\boldsymbol{I}_{L}
$$

Nomeando $\boldsymbol{Z}_{L L}=\left(\boldsymbol{Y}_{L L}-\boldsymbol{Y}_{L T} \boldsymbol{Y}_{T T}^{-1} \boldsymbol{Y}_{T L}\right)^{-1} \in \mathbb{C}^{n_{L} \times n_{L}}$, e $\boldsymbol{K}=$ 
$\boldsymbol{Z}_{L L}\left(\boldsymbol{Y}_{L T} \boldsymbol{Y}_{T T}^{-1} \boldsymbol{Y}_{T G}-\boldsymbol{Y}_{L G}\right) \in \mathbb{C}^{n_{L} \times n_{G}}$, obtém-se finalmente:

$$
\boldsymbol{V}_{L}=\boldsymbol{K} \boldsymbol{V}_{G}-\boldsymbol{Z}_{L L} \boldsymbol{I}_{L}
$$

onde:

$$
\boldsymbol{K} \boldsymbol{V}_{G}=\boldsymbol{E}_{e q}
$$

Deste modo, para uma barra $j$ em análise:

$$
\begin{gathered}
\dot{V}_{L_{j}}=\dot{E}_{e q_{j}}-\bar{Z}_{e q_{j}} \dot{I}_{L_{j}}-\dot{E}_{\text {acoplamento }_{j}} \\
\bar{Z}_{e q_{j}}=\bar{Z}_{L L_{j j}} \\
\dot{E}_{e q_{j}}=\left[K V_{G}\right]_{j} \\
\dot{E}_{\text {acoplamento-j }}=\sum_{\substack{i=1 \\
i \neq j}}^{n} \bar{Z}_{L L_{j i}} \dot{I}_{L_{i}}
\end{gathered}
$$

Nota-se que $\bar{Z}_{e q j}$ representa o equivalente de Thévenin sem considerar a influência das demais cargas, sendo obtido diretamente pelo elemento da diagonal da matriz $\boldsymbol{Z}_{L L}$, tal qual o equivalente "visto" pela barra $j$ utilizada para a análise de curto-circuito. O termo $\dot{E}_{\text {acoplamento-j, por sua vez, representa }}$ o impacto das demais cargas na barra em análise, e sua interpretação deve-se à inserção de uma impedância virtual de acoplamento $\bar{Z}_{c j}$ em série com $\bar{Z}_{e q j}$, como mostrado na Figura 5.4.

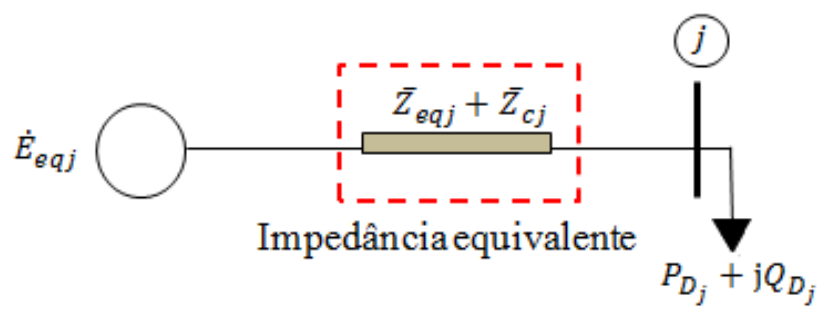

Figura 5.4: Equivalente de porta simples para barra $j$

Deste modo:

$$
\begin{aligned}
\bar{Z}_{c j} & =\frac{\dot{E}_{\text {acoplamento-j }}}{\dot{I}_{L_{j}}}=\sum_{\substack{i=1 \\
i \neq j}}^{n} \bar{Z}_{L L_{j i}} \frac{\dot{I}_{L_{i}}}{\dot{I}_{L_{j}}} \\
& =\sum_{\substack{i=1 \\
i \neq j}}^{n}\left[\bar{Z}_{L L_{j i}}\left(\frac{\dot{S}_{L_{i}}^{*}}{\dot{S}_{L_{j}}^{*}} \times \frac{\dot{V}_{L_{j}}^{*}}{\dot{V}_{L_{i}}^{*}}\right)\right] \\
& =\sum_{\substack{i=1 \\
i \neq j}}^{n}\left[\bar{Z}_{L L_{j i}}\left(\mu_{i j} \times \frac{\dot{V}_{L_{j}}^{*}}{\dot{V}_{L_{i}}^{*}}\right)\right]
\end{aligned}
$$


Então, a impedância de Thévenin é dada por:

$$
\bar{Z}_{j}=\bar{Z}_{e q_{j}}+\bar{Z}_{c j}
$$

O comportamento aproximadamente constante de $\bar{Z}_{c j}$ é avaliado em [25] no sistema IEEE 30 barras com crescimento de carga proporcional, e será mostrado mais adiante. Nestas circunstâncias, é possível observar que tanto $\mu_{i j}$ é constante quanto a relação das tensões nas barras são ligeiramente próximas.

Deste modo, o método CAPS propõe então desmembrar as cargas do sistema multi-portas, considerando explicitamente as influências das cargas entre si na representação dos $n_{L}$ circuitos de porta simples, como mostrado na Figura 5.5. Em seguida, é possível calcular a máxima transferência de potência aparente para cada barra, sua margem associada, e por fim, identificar a barra crítica e a margem de estabilidade do sistema, definida por:

$$
\operatorname{Margem}_{\text {sistema }}=\operatorname{mín}\left\{\operatorname{Margem}_{1}, \ldots, \operatorname{Margem}_{n_{L}}\right\}
$$

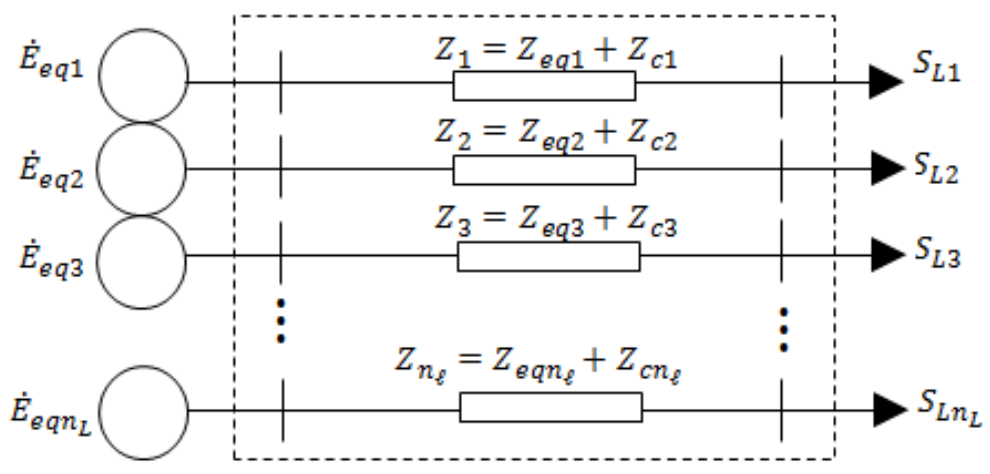

Figura 5.5: Construção dos $n$ circuitos de porta simples

O algoritmo do modelo CAPS é mostrado na Figura 5.6, em que se observa a necessidade das medições das tensões $\dot{V}_{G}$ nos geradores, e dos fasores de tensão $\dot{V}_{L}$ e corrente $\dot{I}_{L}$ nas barras de carga, além de informações a respeito da topologia da rede obtida pelo sistema SCADA ("Supervisory Control And Data Aquisition") para a construção de $\boldsymbol{Y}$. 


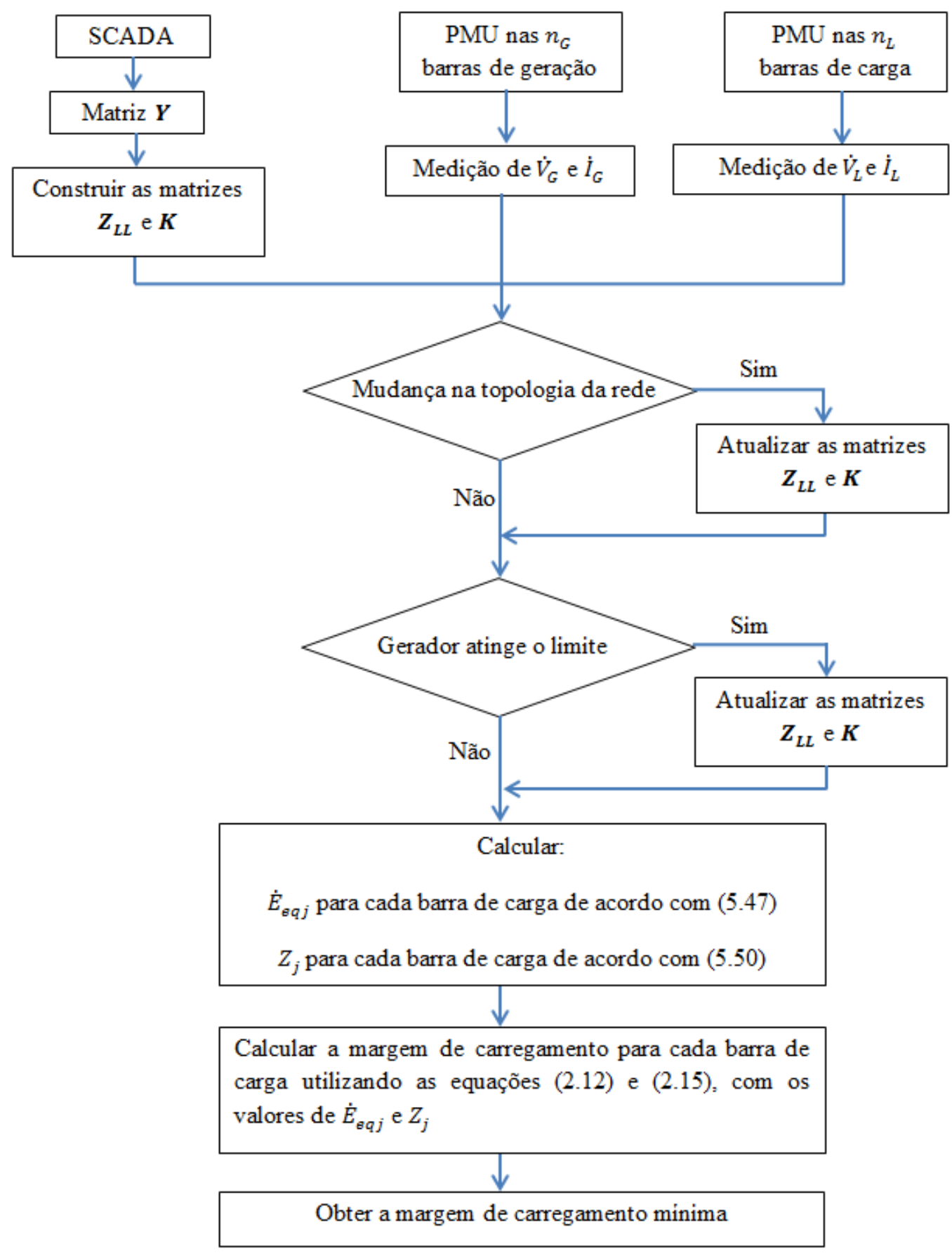

Figura 5.6: Algoritmo do método CAPS

\section{5}

\section{Circuito Acoplado de Porta Simples Aprimorado (CAPS Aprimorado)}

Desde a publicação de [25], diversos artigos buscam contornar imprecisões associados ao método, no que tange à estimativa do máximo carregamento. Em [26], destaca-se que a suposição adotada para a qual o comportamento relativamente constante de $\bar{Z}_{c_{j}}$ não é adequado em cenários de carregamento desproporcional, e se apresenta um método denominado "Circuito Acoplado 
de Porta Simples Modificado" (CAPS Modificado). O método apresentado emprega o conceito de fator de resposta de potência reativa (RPRF - "Reactive Power Response Factor") derivado do equivalente estendido de Ward para compensar as variações do modelo CAPS. Este processo é realizado iterativamente através do cálculo do fator de mitigação $\alpha_{j}$ que corrige a impedância equivalente $\bar{Z}_{j}$ para $\bar{Z}_{j}^{\mathrm{M}}=\alpha_{j} \bar{Z}_{j}$, e implica em uma tensão de Thévenin $\dot{E}_{t h}^{\mathrm{M}}=\dot{V}_{L_{j}}+\left(\alpha_{j} \bar{Z}_{j}\right) \dot{I}_{L_{j}}$.

Posteriormente, em [27], observa-se que em condições topológicas específicas, nas quais as barras de carga são isoladas através de geradores cujo despacho de potência ativa é consideravelmente menor comparado às cargas adjacentes, uma "quebra" no acoplamento pode produzir erros grosseiros na estimativa de margem do método CAPS, e o autor apresenta duas abordagens. A primeira não representa uma nova contribuição ao modelo CAPS, pois sugere a formulação do método CAPS Modificado com ligeira alteração, que deve-se à ampliação no domínio dos valores assumidos pelo fator de mitigação $\alpha_{j}$ para o intervalo $(0, \infty)$. O segundo método destina-se à modelagem dos geradores responsáveis pela quebra do acoplamento como uma barra de carga negativa (Negative Load Model). Contudo, para a segunda abordagem não se define uma metodologia consistente para identificar os geradores responsáveis pela "quebra" de acoplamento.

Mais recentemente, em [28], formulou-se o método CAPS Aprimorado, em que se introduz um fator de sensibilidade $\gamma$ para representar a resposta do sistema à variação de potência ativa, de tal modo que, sendo as medições consecutivas de $\left|\dot{V}_{j}\right|$ e $P_{j}$ nos instantes $t_{i}$ e $t_{i+1}$ suficientemente próximos para que seja assumida a relação:

$$
\gamma_{j}=\frac{d P_{j}}{d\left|\dot{V}_{j}\right|}=\frac{P_{j}\left(t_{i}\right)-P_{j}\left(t_{i+1}\right)}{\left|\dot{V}_{j}\left(t_{i}\right)\right|-\left|\dot{V}_{j}\left(t_{i+1}\right)\right|}
$$

o modelo busca determinar os parâmetros $\dot{E}_{e q_{j}}^{\prime}$ e $Z_{e q_{j}}^{\prime}$ que satisfazem a equação (5.52) e a equação associada ao fluxo de carga no circuito equivalente, dada por:

$$
\left|\dot{V}_{j}\right|^{4}-\left[\left|\dot{E}_{e q_{j}}^{\prime}\right|^{2}-2\left(R_{j}^{\prime} P_{j}+X_{j}^{\prime} Q_{j}\right)\right]\left|\dot{V}_{j}\right|^{2}+\left(P_{j}^{2}+Q_{j}^{2}\right)\left(R_{j}^{\prime 2}+X_{j}^{\prime 2}\right)=0
$$

Tomando a equação (5.53) como $\Phi\left(\left|\dot{V}_{j}\right|, P_{j}\right)$, obtém-se através da diferenciação total:

$$
d \Phi\left(\left|\dot{V}_{j}\right|, P_{j}\right)=\frac{\partial d \Phi\left(\left|\dot{V}_{j}\right|, P_{j}\right)}{\partial P_{j}} d P_{j}+\frac{\partial d \Phi\left(\left|\dot{V}_{j}\right|, P_{j}\right)}{\partial\left|\dot{V}_{j}\right|} d\left|\dot{V}_{j}\right|=0
$$

donde segue que: 


$$
\gamma_{j}=\frac{d P_{j}}{d\left|\dot{V}_{j}\right|}=-\frac{\partial \Phi\left(\left|\dot{V}_{j}\right|, P_{j}\right) / \partial\left|\dot{V}_{j}\right|}{\partial \Phi\left(\left|\dot{V}_{j}\right|, P_{j}\right) / \partial P_{j}}
$$

A demonstração de $\partial \Phi\left(\left|\dot{V}_{j}\right|, P_{j}\right) / \partial\left|\dot{V}_{j}\right|$ e $\partial \Phi\left(\left|\dot{V}_{j}\right|, P_{j}\right) / \partial P_{j}$ podem ser encontradas no Apêndice $\mathrm{A}$, donde segue que:

$$
\frac{\partial \Phi\left(\left|\dot{V}_{j}\right|, P_{j}\right)}{\partial\left|\dot{V}_{j}\right|}=-2\left|\dot{V}_{j}\right|\left|\dot{E}_{e q_{j}}\right|\left(\left|\dot{E}_{e q_{j}}\right|-2\left|\dot{V}_{j}\right| \cos \left(\theta_{e}-\theta\right)\right)
$$

e

$$
\frac{\partial \Phi\left(\left|\dot{V}_{j}\right|, P_{j}\right)}{\partial P_{j}}=\left[\frac{2\left|\dot{V}_{j}\right|^{2}\left|\dot{E}_{e q_{j}}\right|}{P_{j}}\right]\left(\left|\dot{E}_{e q_{j}}\right|-\left|\dot{V}_{j}\right| \cos \left(\theta_{e}-\theta\right)\right)
$$

Substituindo (5.56) e (5.57) em (5.55), é possível obter o módulo da tensão de Thévenin $\left|\dot{E}_{e q_{j}}^{\prime}\right|$ :

$$
\left|\dot{E}_{e q_{j}}^{\prime}\right|=\left(\frac{2-\gamma_{j}\left|\dot{V}_{j}\right| / P_{j}}{1-\gamma_{j}\left|\dot{V}_{j}\right| / P_{j}}\right)\left|\dot{V}_{j}\right| \cos \left(\theta_{e}-\theta\right)
$$

em que $\theta$ é o ângulo do fasor de tensão $\dot{V}_{j}$, e $\theta_{e}$ representa o ângulo de $\left|\dot{E}_{e q_{j}}^{\prime}\right|$, fornecido por:

$$
\theta_{e}=\angle\left[K V_{G}\right]_{j}
$$

A impedância de $\bar{Z}_{j}^{\prime}$ é imediatamente obtida, fazendo:

$$
\bar{Z}_{j}^{\prime}=\frac{\dot{E}_{e q_{j}}^{\prime}-\dot{V}_{j}}{\dot{I}_{j}}
$$

O algoritmo proposto foi amplamente testado em [28] para estimar a margem de diversos sistemas, podendo ser implementado através dos seguintes passos:

CAPS Aprimorado
Passo 1) Construir a matriz $\boldsymbol{K}$ a partir da matriz
de admitância $\boldsymbol{Y}$
Passo 2) Calcular o ângulo de $\dot{E}_{e q_{j}}$ "visto"pela
barra $j$ pela equação $(5.59)$
Passo 3) Calcular a sensibilidade $\gamma_{j}$ da potência
ativa $P_{j}$ com relação a $\left|\dot{V}_{j}\right|$ de acordo com $(5.52)$
Passo 4) Calcular $\left|\dot{E}_{e q_{j}}^{\prime}\right|$ através da equação $(5.58)$
Passo 5) Calcular a impedância $\bar{Z}_{j}^{\prime}$ por $(5.60)$
Passo 6) Calcular a margem de carregamento para cada barra de
carga $j$ considerando as equações $(2.12)$ e $(2.15)$
Passo 7) Calcular a margem de carregamento do sistema, definida
por (5.51)


O fator de sensibilidade $\gamma_{j}$, como fora definido, captura a resposta do sistema a variações de potência ativa das cargas. No entanto, o algoritmo pode ser reformulado tendo em vista as variações de potência reativa $Q_{j}$. Nota-se, durante o cenário de crescimento de carga, que $\gamma_{j} \leq 0$, ocorrendo a igualdade na condição de máximo carregamento, onde a equação (5.58) reduz-se a:

$$
\left|\dot{E}_{e q_{j}}^{\prime}\right|=2\left|\dot{V}_{j}\right| \cos \left(\theta_{e}-\theta\right)
$$

Deste modo, a implementação do método CAPS Aprimorado, tal como os métodos CAPS e CAPS Modificado, também necessita do conhecimento da topologia da rede para a construção das matrizes $\boldsymbol{Z}_{L L}$ e $\boldsymbol{K}$, e dos fasores de tensão $\dot{V}_{G}$ nos geradores. No entanto, apenas as medições de corrente e tensão na barra de carga em análise são necessárias, o que representa uma importante contribuição aos modelos multi-portas, dada a redução na complexidade de medição. Ademais, comparado aos métodos existentes, o algoritmo exije menor custo computacional.

\subsection{1}

\section{Avaliação de Barras de Transferência}

Para a avaliação deste conjunto de barras, assume-se que a carga conectada à barra de transferência $i$ é idêntica à soma dos fluxos que "deixam" a barra em direção às barras adjacentes $\Omega_{i}$. Deste modo, a barra em análise é avaliada como uma barra de carga.

Como as conexões entre a barra $i$ em análise e o conjunto $\Omega_{i}$ a ela associada são virtualmente "quebradas", a matriz de admitância de barra $\boldsymbol{Y}^{\text {fic }}$ deve ser previamente processada, considerando uma topologia fictícia. A análise deste conjunto de barras será analisada em maiores detalhes no Capítulo 6, considerando o sistema IEEE 30 barras.
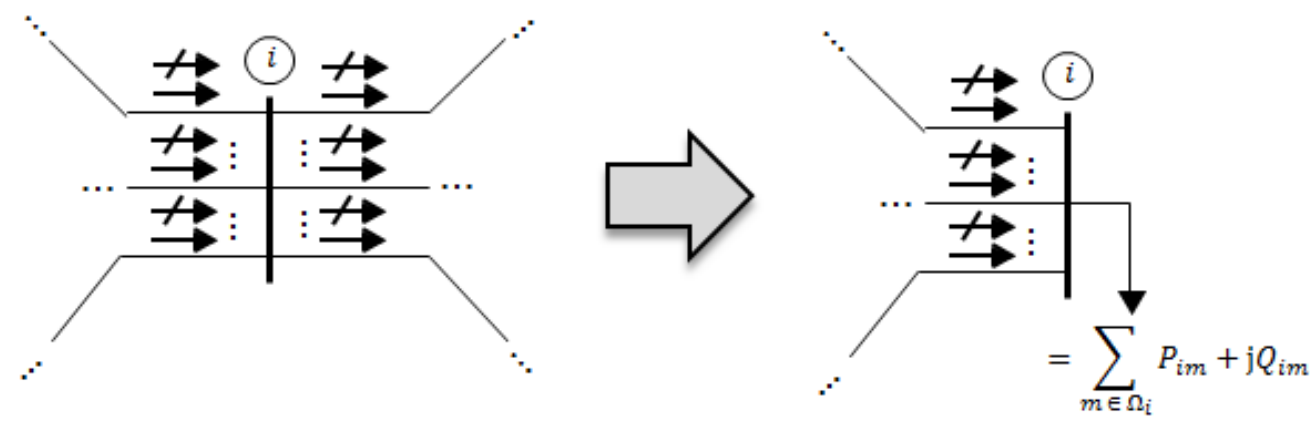

Figura 5.7: Avaliação das barras de transferência 


\section{5 .2}

\section{Avaliação de Barras de Tensão Controlada : Uma Contribuição a partir do Modelo CAPS Aprimorado}

Uma vez justificada a importância do monitoramento das barras de geração, esta seção destina-se a aplicar o método CAPS Aprimorado para este conjunto de barras se valendo, principalmente, da flexibilidade do modelo multi-portas que permite, com base nas expressões (5.36) a (5.38), tomar um caminho análogo a fim de estimar o equivalente "visto" pela barra terminal do gerador. Deste modo, após algumas manipulações algébricas chega-se à expressão:

$$
\left(\boldsymbol{Y}_{G G}-\boldsymbol{Y}_{G T} \boldsymbol{Y}_{T T}^{-1} \boldsymbol{Y}_{T G}\right) \boldsymbol{V}_{G}=\left(\boldsymbol{Y}_{G T} \boldsymbol{Y}_{T T}^{-1} \boldsymbol{Y}_{T L}-\boldsymbol{Y}_{G L}\right) \boldsymbol{V}_{L}+\boldsymbol{I}_{G}
$$

nomeando as matrizes $\boldsymbol{Z}_{G G}=\left(\boldsymbol{Y}_{G G}-\boldsymbol{Y}_{G T} \boldsymbol{Y}_{T T}^{-1} \boldsymbol{Y}_{T G}\right)^{-1} \in \mathbb{C}^{n_{G} \times n_{G}}$, e $\boldsymbol{K}_{G}=$ $\boldsymbol{Z}_{G G}\left(\boldsymbol{Y}_{G T} \boldsymbol{Y}_{T T}^{-1} \boldsymbol{Y}_{T L}-\boldsymbol{Y}_{G L}\right) \in \mathbb{C}^{n_{L} \times n_{G}}$, então:

$$
\boldsymbol{V}_{G}=\boldsymbol{K}_{G} \boldsymbol{V}_{L}+\boldsymbol{Z}_{G G} \boldsymbol{I}_{G}
$$

onde:

$$
\boldsymbol{E}_{e q}=\boldsymbol{K}_{G} \boldsymbol{V}_{L}
$$

Convenientemente em [28], se explicitou a sensibilidade $\gamma$ em função da variação de potência ativa em relação à variação do módulo da tensão, pois os autores consideram em sua análise o aumento de carga mantendo-se o fator de potência constante.

Contudo, para o monitoramento das barras de geração, adotar a sensibilidade como a variação de potência reativa em relação à variação do módulo de tensão mostrou-se mais conviente, permitindo a análise de compensadores síncronos.

Deste modo, se utilizará a sensibilidade $\psi_{k}$ para a barra de geração $k$ como:

$$
\psi_{k}=\frac{d Q_{k}}{d\left|\dot{V}_{k}\right|}=\frac{Q_{k}\left(t_{i}\right)-Q_{k}\left(t_{i+1}\right)}{\left|\dot{V}_{k}\left(t_{i}\right)\right|-\left|\dot{V}_{k}\left(t_{i+1}\right)\right|}
$$

as derivadas parciais $\partial \Phi\left(\left|\dot{V}_{k}\right|, Q_{k}\right) / \partial\left|\dot{V}_{k}\right|$ e $\partial \Phi\left(\left|\dot{V}_{k}\right|, Q_{k}\right) / \partial Q_{k}$ serão, respectivamente:

$$
\frac{\partial \Phi\left(\left|\dot{V}_{k}\right|, Q_{k}\right)}{\partial\left|\dot{V}_{k}\right|}=-2\left|\dot{V}_{k}\right|\left|\dot{E}_{e q_{k}}\right|\left(\left|\dot{E}_{e q_{k}}\right|-2\left|\dot{V}_{k}\right| \cos \left(\theta_{e}-\theta\right)\right)
$$

e

$$
\frac{\partial \Phi\left(\left|\dot{V}_{k}\right|, Q_{k}\right)}{\partial Q_{k}}=\left[\frac{2\left|\dot{V}_{k}\right|^{2}\left|\dot{E}_{e q_{k}}\right|}{Q_{k}}\right]\left(\left|\dot{E}_{e q_{k}}\right|-\left|\dot{V}_{k}\right| \cos \left(\theta_{e}-\theta\right)\right)
$$

Logo, a tensão de Thévenin "vista" pela barra de geração será: 


$$
\left|\dot{E}_{e q_{k}}^{\prime}\right|=\left(\frac{2-\psi_{k}\left|\dot{V}_{k}\right| / Q_{k}}{1-\psi_{k}\left|\dot{V}_{k}\right| / Q_{k}}\right)\left|\dot{V}_{k}\right| \cos \left(\theta_{e}-\theta\right)
$$

sendo o ângulo $\theta_{e}$ obtido por:

$$
\theta_{e}=\angle\left[K_{G} V_{L}\right]_{k}
$$

e a impedância $\bar{Z}_{k}^{\prime}$ :

$$
\bar{Z}_{k}^{\prime}=\frac{\dot{E}_{e q_{k}}^{\prime}-\dot{V}_{k}}{\dot{I}_{k}}
$$




\section{6 \\ Resultados}

Os métodos descritos no Capítulo 5 serão confrontados em diversos sistemas didáticos encontrados na literatura, com o propósito de avaliar seus desempenhos na identificação da máxima transferência de potência (MTP).

Este capítulo busca objetivamente:

- Comparar a utilização dos equivalente multi-portas como índices de avaliação das condições de estabilidade de tensão, aos métodos apresentado no Capítulo 4;

- Expor, através de um conjunto de análises, as principais limitações dos modelos;

- Avaliar a aplicabilidade das modificações proposta ao método CAPS aprimorado quanto às barras de transferência e de geração, e investigar a coerência dos seus resultados;

Destaca-se que maior ênfase será dada aos métodos de Corsi-Taranto e CAPS Aprimorado devido, principalmente, à superioridade destes métodos e suas característas seminais.

\section{1}

\section{Identificação da Barra de Carga Crítica}

Considerando a utilização dos modelos multi-portas como índices de avaliação das condições de estabilidade de tensão, pretende-se inicialmente analisar a capacidade dos modelos CAPS e CAPS Aprimorado de identificarem as barras de carga crítica quando confrontados com os métodos da matriz $\boldsymbol{D}^{\prime}$ [15], e pela análise dos autovalores e autovetores [16]. Para tanto, considera-se inicialmente o sistema teste IEEE 14 barras, mostrado na Figura 6.1, que representa uma parcela do sistema estadunidense, em fevereiro de 1962.

Simulando o caso base no algoritmo de fluxo de carga desenvolvido no software MATLAB, obtém-se como resultado os dados de barra apresentados na Tabela 6.1. O procedimento para a identificação da barra crítica inicializase imediatamente após a execução do fluxo de carga, através dos métodos da matriz $\boldsymbol{D}^{\prime}$ e da matriz reduzida $\boldsymbol{J}_{R}^{Q}$, aproveitando os dados remanescentes da simulação. Deste modo, como pode ser observado na Tabela 6.2, as barra de 
carga crítica 9 e 14, identificadas pelo método da matriz $\boldsymbol{D}^{\prime}$, apresentam margens de carregamento iguais a 94,87\% e 95,65 \%, respectivamente. Portanto, devido a pequena diferença de $0,78 \%$, é razoável considera-las como as barras de carga crítica do sistema.

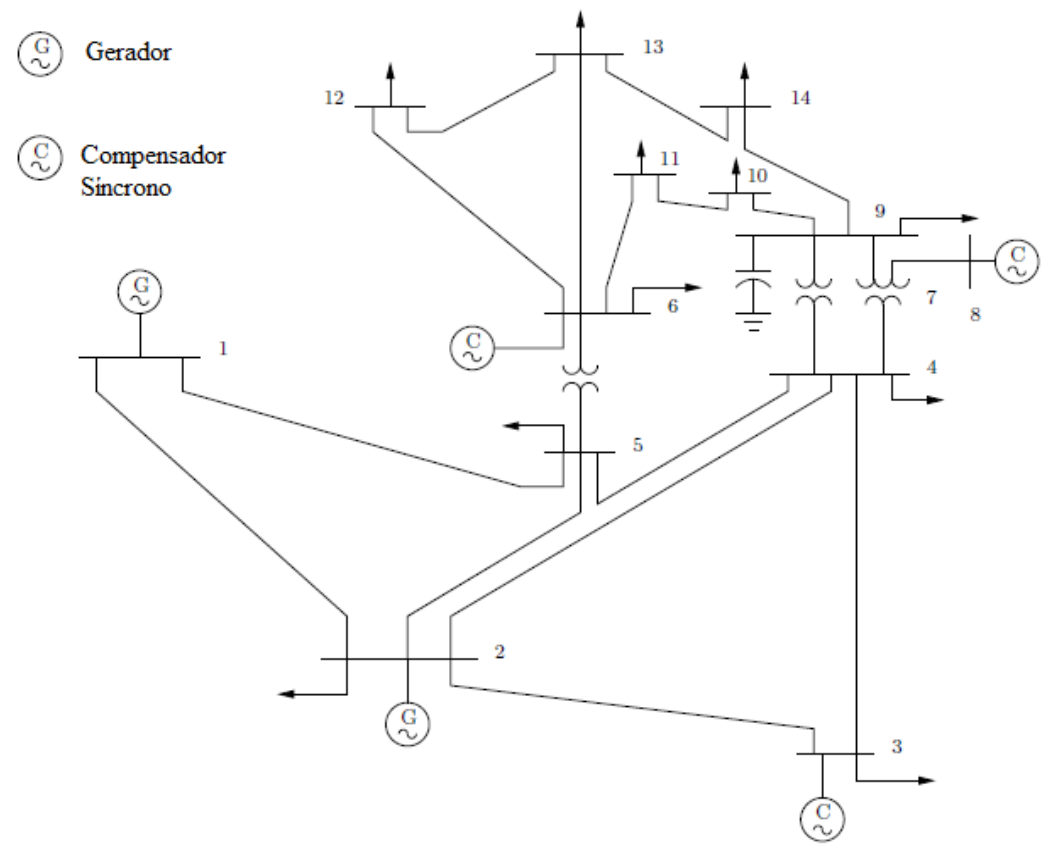

Figura 6.1: Sistema IEEE 14 barras

Tabela 6.1: Dados de barra - Sistema IEEE 14 barras

\begin{tabular}{|ccccccccc|}
\hline Barra & Tipo & $|\dot{V}|[\mathrm{pu}]$ & $\theta\left[{ }^{\circ}\right]$ & $P_{G}[\mathrm{pu}]$ & $Q_{G}[\mathrm{pu}]$ & $P_{L}[\mathrm{pu}]$ & $Q_{L}[\mathrm{pu}]$ & $b_{k}^{s h}[\mathrm{pu}]$ \\
\hline \hline 1 & $V \theta$ & 1,060 & 0,000 & 2,324 & $-0,169$ & & & \\
2 & $P V$ & 1,045 & $-4,981$ & 0,400 & 0,424 & 0,217 & 0,127 & \\
3 & $P V$ & 1,010 & $-12,718$ & & 0,234 & 0,942 & 0,190 & \\
4 & $P Q$ & 1,019 & $-10,324$ & & & 0,478 & $-0,039$ & \\
5 & $P Q$ & 1,020 & $-8,783$ & & & 0,076 & 0,016 & \\
6 & $P V$ & 1,070 & $-14,223$ & 0,000 & 0,122 & 0,112 & 0,075 & \\
7 & $P Q$ & 1,062 & $-13,368$ & & & & & \\
8 & $P V$ & 1,090 & $-13,368$ & 0,000 & 0,174 & & & \\
9 & $P Q$ & 1,056 & $-14,947$ & & & 0,295 & 0,166 & 0,19 \\
10 & $P Q$ & 1,051 & $-15,104$ & & & 0,090 & 0,058 & \\
11 & $P Q$ & 1,057 & $-14,795$ & & & 0,035 & 0,018 & \\
12 & $P Q$ & 1,055 & $-15,078$ & & & 0,061 & 0,016 & \\
13 & $P Q$ & 1,050 & $-15,159$ & & & 0,135 & 0,058 & \\
14 & $P Q$ & 1,036 & $-16,039$ & & & 0,149 & 0,050 & \\
\hline \hline Total & & & & & & & \\
\hline
\end{tabular}


Tabela 6.2: Método da Matriz $\boldsymbol{D}^{\prime}$ - Sistema IEEE 14 barras

\begin{tabular}{|cccccc|}
\hline Barra & Ordenação & $S_{i}[\mathrm{MVA}]$ & $S_{m}[\mathrm{MVA}]$ & Margem [\%] & $\beta\left[^{\circ}\right]$ \\
\hline \hline $2^{\dagger}$ & 10 & 34,88 & 2488,74 & 98,60 & 91,85 \\
$3^{\dagger}$ & 1 & 94,30 & 771,08 & 87,77 & 95,85 \\
4 & 5 & 47,96 & 1574,26 & 96,95 & 93,12 \\
5 & 12 & 7,77 & 1648,70 & 99,53 & 91,26 \\
$6^{\dagger}$ & 6 & 12,16 & 488,11 & 97,51 & 99,45 \\
7 & 13 & 0,00 & 795,92 & 100,00 & 90,27 \\
$8^{\dagger}$ & 2 & 17,36 & 320,60 & 94,59 & 92,43 \\
9 & 3 & 33,85 & 660,42 & 94,87 & 91,70 \\
10 & 8 & 10,71 & 523,53 & 97,95 & 91,57 \\
11 & 11 & 3,94 & 513,98 & 99,23 & 98,25 \\
12 & 9 & 6,31 & 436,31 & 98,55 & 105,78 \\
13 & 7 & 14,69 & 621,92 & 97,64 & 104,91 \\
14 & 4 & 15,72 & 361,51 & 95,65 & 92,76 \\
\hline
\end{tabular}

$\dagger$ Barra de tensão controlada

Utilizando o método baseado na avaliação dos autovalores e autovetores é possível identificar a partir da Figura 6.2(a) a barra 14 como crítica através do seu maior fator de participação no modo crítico. A escolha da barra 14 como crítica é reiterada pela análise do método CAPS tomando o ponto de operação do caso base, e pelo método CAPS Aprimorado considerando dois pontos de operação ligeiramente próximos, conforme observado nas Figuras 6.2(b) e 6.2(c). A margem de carregamento do sistema obtida pelo fluxo de potência continuado (FPC) é traçada como referência, sendo computada no software ANAREDE, pois requer uma formulação parametrizada do fluxo de carga convencional. 


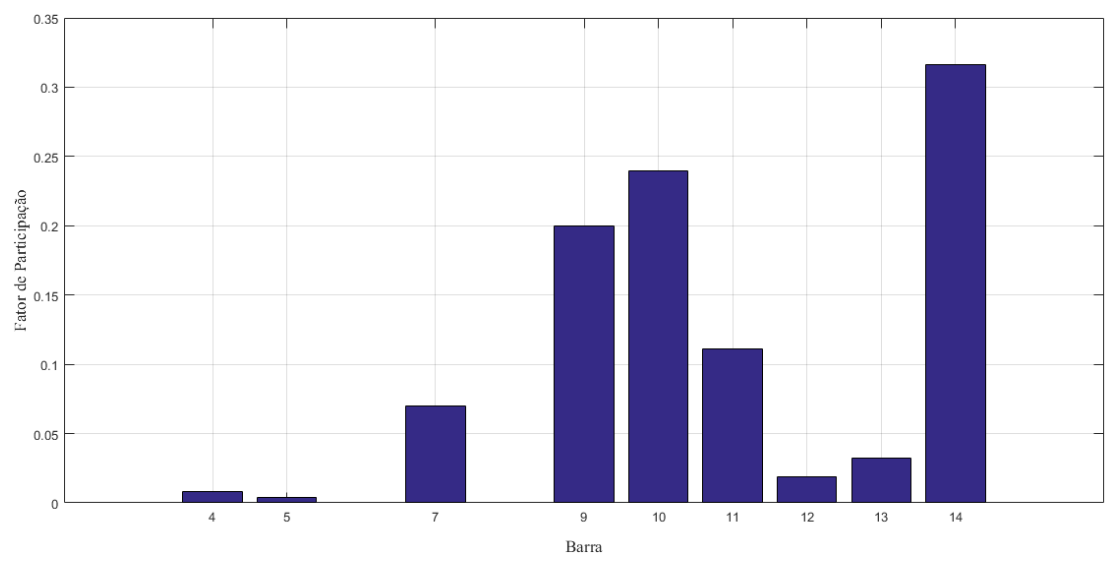

6.2(a): Identificação da barra crítica pela matriz $\boldsymbol{J}_{R}^{Q}$

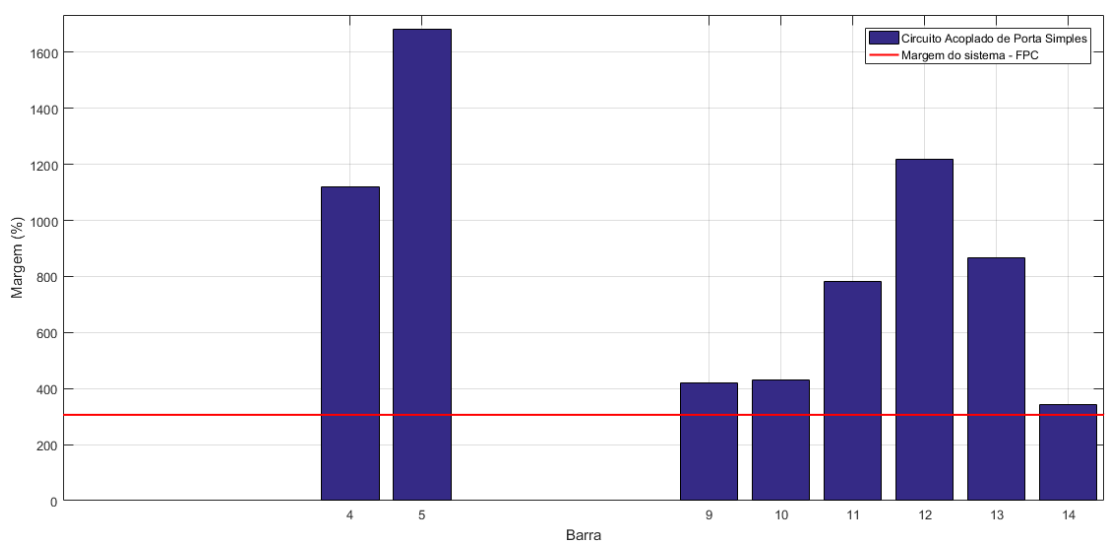

6.2(b): Margem estimada pelo método CAPS

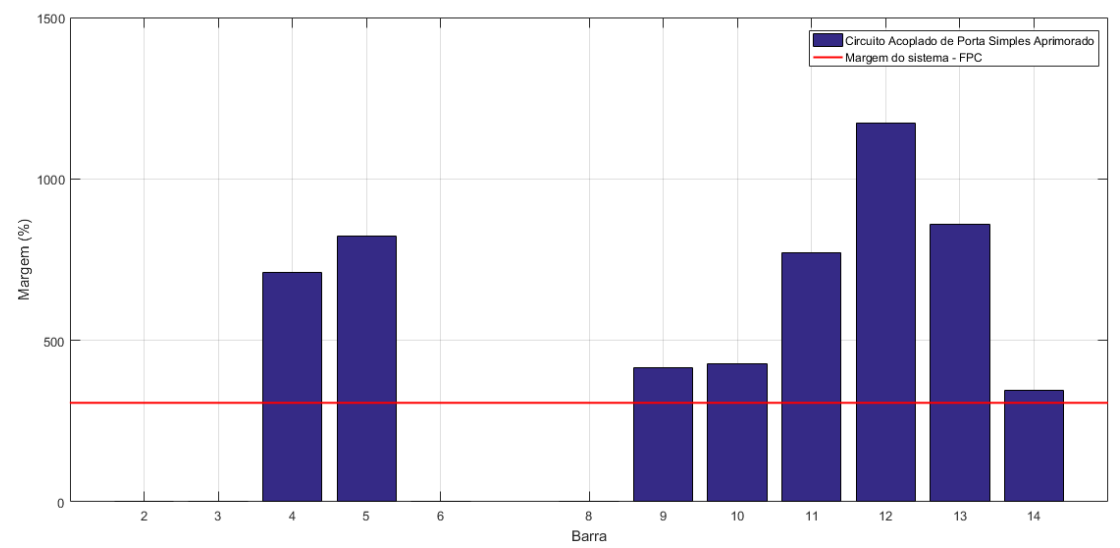

6.2(c): Margem estimada pelo método CAPS Aprimorado

Figura 6.2: Identificação da barra de carga crítica no sistema IEEE 14 barras

Para validação mais ampla dos métodos, a Tabela 6.3 apresenta diversos sistemas testes aos quais foram submetidos os métodos CAPS e CAPS Aprimorado, considerando os respectivos casos base. Conforme mencionado no Capítulo 4, o método da matriz $\boldsymbol{D}^{\prime}$ é capaz de avaliar as barras de tensão controlada do sistema, que muitas vezes manisfestam-se como as barras de menor margem. Deste modo, sem desprezar as informações referentes às barras 
de geração, mas tendo em vista a comparação a respeito da identificação das barras de carga crítica, a Tabela 6.3 organiza-se elencando as barras de menor margem, de tal modo a capturar as três primeiras barras de carga consideradas críticas.

Tabela 6.3: Identificação da barra crítica

\begin{tabular}{|c|c|c|c|c|}
\hline Sistema & $\boldsymbol{J}_{R}^{Q}$ & $D^{\prime}$ & CAPS & CAPS Aprimorado \\
\hline "Stagg \& El-Albiad ${ }^{1}$ & 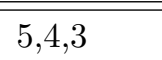 & $5,3,4$ & $5,4,3$ & $5,4,3$ \\
\hline WSCC 9 Barras & $5,6,8$ & $2^{\dagger}, 3^{\dagger}, 5,8,6$ & $5,6,8$ & $5,6,8$ \\
\hline IEEE 14 Barras & $14,10,9$ & $3^{\dagger}, 8^{\dagger}, 9,14,4$ & $14,9,10$ & $14,9,10$ \\
\hline IEEE 30 Barras & $30,29,26$ & $5^{\dagger}, 30,11^{\dagger}, 21,26$ & $30,26,29$ & $30,26,29$ \\
\hline New England ${ }^{2}$ & $12,7,8$ & $\begin{array}{l}38^{\dagger}, 36^{\dagger}, 34^{\dagger}, 35^{\dagger}, 33^{\dagger}, 32^{\dagger} \\
37^{\dagger}, 20,30,8\end{array}$ & $15,4,8$ & $8,7,4$ \\
\hline IEEE 57 Barras & $31,33,32$ & $8^{\dagger}, 18,31,53$ & $31,33,32$ & $31,33,32$ \\
\hline Nordic $^{3}$ & $1,1041,3$ & $\begin{array}{l}\mathrm{g} 19^{\dagger}, \mathrm{g} 16^{\dagger}, \mathrm{g} 8^{\dagger}, \mathrm{g} 6^{\dagger}, \mathrm{g} 4^{\dagger} \\
\mathrm{g} 16^{\dagger}, 1,51,4\end{array}$ & $1,3,5$ & $1,3,4$ \\
\hline $\begin{array}{l}{ }^{\dagger} \text { Barras de tensão cc } \\
{ }^{1} \text { Sistema } 5 \text { barras ex } \\
{ }^{2} \text { Sistema } 39 \text { barras } \\
{ }^{3} \text { Sistema } 74 \text { barras }\end{array}$ & $\begin{array}{l}\text { ntrolada } \\
\text { traído de [ } \\
\text { xtraído de } \\
\text { xtraído de }\end{array}$ & $\begin{array}{l}29] \\
{[30]} \\
{[31]}\end{array}$ & & \\
\hline
\end{tabular}

Com efeito, observa-se pela Tabela 6.3 que os métodos CAPS e CAPS Aprimorado identificam na maioria dos casos a barra de carga crítica em consonância com os métodos da matriz $\boldsymbol{D}^{\prime}$ e da matriz reduzida $\boldsymbol{J}_{R}^{Q}$. Naturalmente, necessita-se avaliar a margem estimada considerando o ponto de operação do caso base. Para tanto, [25, 28], balizam-se na margem fornecida pelo FPC e, deste modo, tomando a dita margem como referência na Tabela 6.4 (considerando os limites dos geradores em aberto), calcula-se o erro relativo percentual $\epsilon_{r}[\%]$ das margens estimadas pelos modelos, em que se nota pela maioria dos casos analisados que o método CAPS Aprimorado oferece menor erro ao estimar a margem de carregamento do sistema.

Tabela 6.4: Comparação entre as margens estimadas no caso base

\begin{tabular}{|llllcl|}
\hline Sistema & FPC [\%] & \multicolumn{2}{c|}{ Margem estimada } & \multicolumn{2}{c|}{ Margem estimada } \\
& & CAPS [\%] & $\epsilon_{r}[\%]$ & CAPS Aprimorado [\%] & $\epsilon_{r}[\%]$ \\
\hline \hline Stagg \& El-Albiad & 202,66 & 225,70 & 11,37 & 189,12 & 6,68 \\
WSCC 9 Barras & 137,39 & 161,46 & 17,52 & 194,04 & 41,23 \\
IEEE 14 Barras & 300,44 & 341,09 & 13,53 & 344,52 & 14,67 \\
IEEE 30 Barras & 195,04 & 168,74 & 13,48 & 166,70 & 14,53 \\
New England & 49,12 & 124,42 & 153,30 & 101,64 & 106,92 \\
IEEE 57 Barras & 78,54 & 48,24 & 38,58 & 55,08 & 29,87 \\
Nordic & 3,20 & 5,48 & 71,25 & 2,82 & 11,87 \\
\hline
\end{tabular}




\section{2}

\section{Avaliação das Barras de Carga}

A rede escolhida para comparação dos métodos na avaliação da barra de carga corresponde ao sistema WSCC (Western System Coordinating Council) 9 barras, visto na Figura 6.3, em que o ponto de operação inicial é apresentado na Tabela 6.5 , e a margem estimada pelo método da matriz $\boldsymbol{D}^{\prime}$ encontra-se na Tabela 6.6.

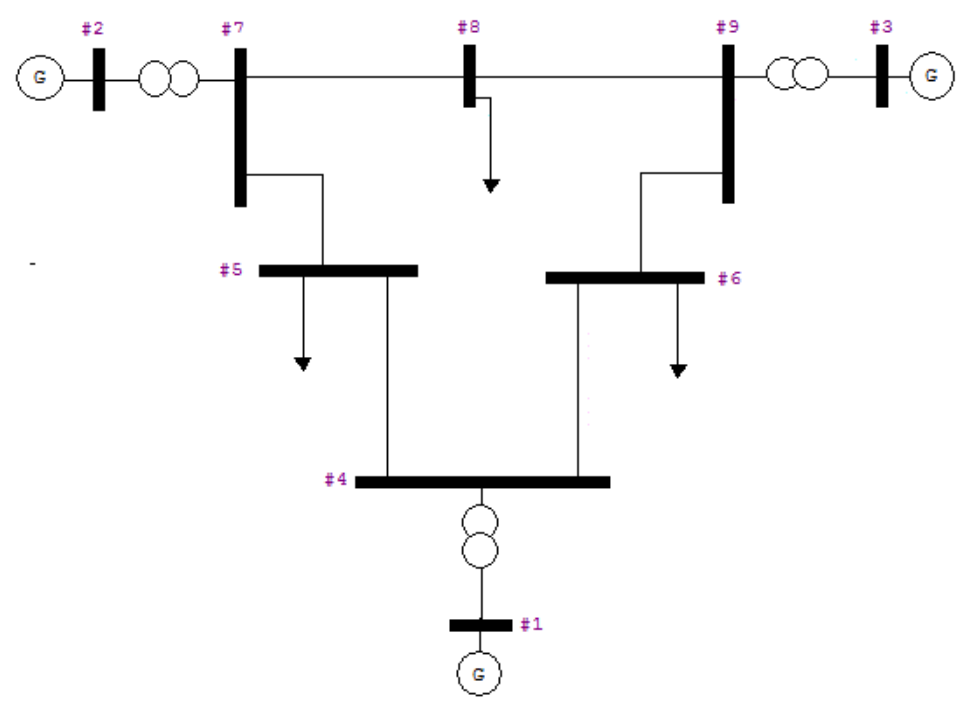

Figura 6.3: Sistema WSCC 9 barras

Tabela 6.5: Dados de barra - Sistema WSCC 9 barras

\begin{tabular}{|c|c|c|c|c|c|c|c|c|}
\hline Barra & Tipo & $\dot{V} \mid[\mathrm{pu}]$ & $\theta\left[^{\circ}\right]$ & $P_{G}[\mathrm{pu}]$ & $Q_{G}[\mathrm{pu}]$ & $P_{L}[\mathrm{pu}]$ & $Q_{L}[\mathrm{pu}]$ & $b_{k}^{s h}[\mathrm{pu}]$ \\
\hline 1 & $V \theta$ & 1,040 & 0,000 & 0,716 & 0,270 & & & \\
\hline 2 & $P V$ & 1,025 & 9,280 & 1,630 & 0,067 & & & \\
\hline 3 & $P V$ & 1,025 & 4,665 & 0,850 & $-0,109$ & & & \\
\hline 4 & $P Q$ & 1,026 & $-2,217$ & & & & & \\
\hline 5 & $P Q$ & 0,996 & $-3,989$ & & & 1,250 & 0,500 & \\
\hline 6 & $P Q$ & 1,013 & $-3,687$ & & & 0,900 & 0,300 & \\
\hline 7 & $P Q$ & 1,026 & 3,730 & & & & & \\
\hline 8 & $P Q$ & 1,016 & 0,727 & & & & & \\
\hline 9 & $P Q$ & 1,032 & 1,967 & & & 1,000 & 0,350 & \\
\hline Total & & & & 3,196 & 0,228 & 3,150 & 1,150 & \\
\hline
\end{tabular}


Tabela 6.6: Método da Matriz $\boldsymbol{D}^{\prime}$ - Sistema WSCC 9 barras

\begin{tabular}{|cccccc|}
\hline Barra & Ordenação & $S_{i}[\mathrm{MVA}]$ & $S_{m}[\mathrm{MVA}]$ & Margem [\%] & $\beta\left[^{\circ}\right]$ \\
\hline \hline $2^{\dagger}$ & 1 & 163,14 & 448,46 & 63,62 & 117,36 \\
$3^{\dagger}$ & 2 & 85,69 & 434,81 & 80,29 & 105,56 \\
4 & 8 & 0,00 & 2097,55 & 100,00 & 93,98 \\
5 & 3 & 134,63 & 933,89 & 85,58 & 96,01 \\
6 & 5 & 94,87 & 917,85 & 89,66 & 93,66 \\
7 & 6 & 0,00 & 1077,15 & 100,00 & 104,45 \\
8 & 4 & 105,95 & 814,33 & 86,99 & 94,25 \\
9 & 7 & 0,00 & 1098,97 & 100,00 & 101,91 \\
\hline
\end{tabular}

† Barra de tensão controlada

Para esta simulação, realizaram-se sucessivos fluxos de potência com aumentos proporcionais e desproporcionais nos patamares de carga, consideradas como do tipo potência constante, até sua insolubilidade. Os resultados apresentados referem-se à barra 5 , identificada como a barra de carga crítica, e os casos encontrados na Tabela 6.7 foram escolhidos com o propósito de se avaliar o desempenho dos métodos na identificação dos parâmetros do equivalente de Thévenin e da margem associada à barra de carga em análise.

Tabela 6.7: Variações percentuais de carga no sistema WSCC 9 barras

\begin{tabular}{|cccc|}
\hline Caso & Barra & $\Delta P_{L}[\%]$ & $\Delta Q_{L}[\%]$ \\
\hline \hline 1 & $5,6,8$ & 0,01 & 0,01 \\
\hline \hline & 5 & 0,03 & 0,03 \\
2 & 6 & 0,05 & 0,05 \\
& 8 & 0,02 & 0,02 \\
\hline
\end{tabular}

Adotou-se para o método de ŠVG a tolerância $|\Delta \dot{I}|^{\text {mín }}$ igual a $10^{-5}$. A

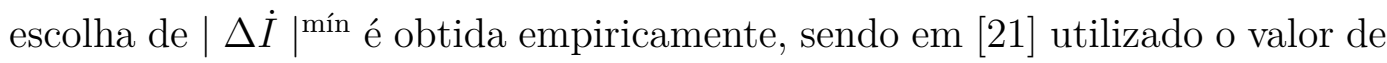
0,015 para simulação dinâmica, e em [10] o valor de 0,0005 para simulações de fluxo de potência com variação nos incrementos de carga. Deve-se ressaltar que, se por um lado a escolha muito pequena do valor de tolerância pode promover pontos de singularidade que não fornecerão um valor de impedância, a escolha de valores muito grandes pode produzir uma estimação dos parâmetros não condizente com o ponto de operação atual, pois neste caso a impedância estimada conserva seu valor anterior, ou seja $\bar{Z}_{t h}^{i}=\bar{Z}_{t h}^{i-1}$.

Considerando então o caso 1 foi possível rastrear, pela maioria dos métodos apresentados, o ponto de MTP, observado na Figura 6.4 através do gráfico de impedâncias. Nota-se também que as curvas de impedância em [pu] 
estimadas pelos métodos VIP e ŠVG encontram-se sobrepostas.

A pequena diferença associada à previsão de máximo carregamento devese às características do FPC, que não apresenta solução nas proximidades do LET.

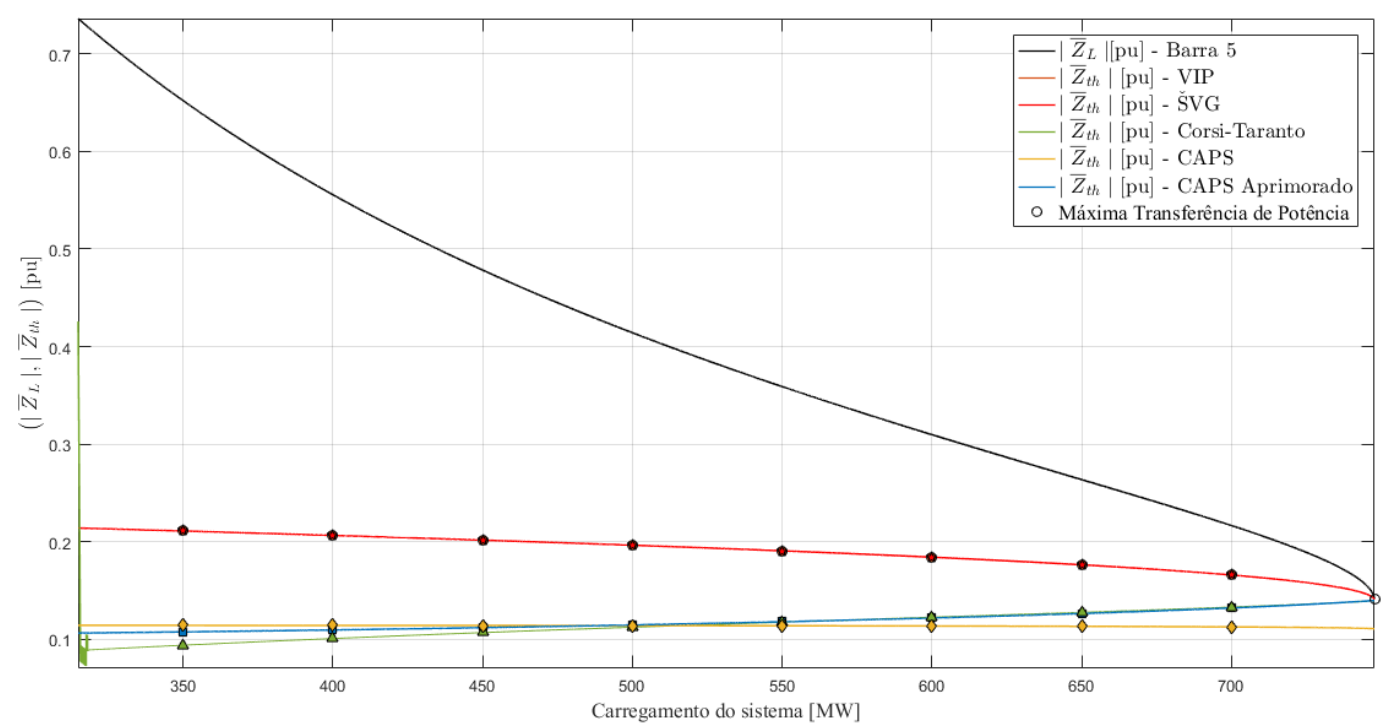

Figura 6.4: Gráfico de impedância em [pu] - caso 1

Importante destacar na Figura 6.5, a identificação da resistência e reatância de Thévenin realizada pelo método CAPS Aprimorado, que permite concluir que a premissa $X_{t h}>>R_{t h}$ não é válida para todo ponto de operação, e ainda que não comprometa a convergência do método de Corsi-Taranto nos casos avaliados, deve-se ter em mente que a redução na relação $X_{t h} / R_{t h}$ tende a deteriorar seu desempenho. 


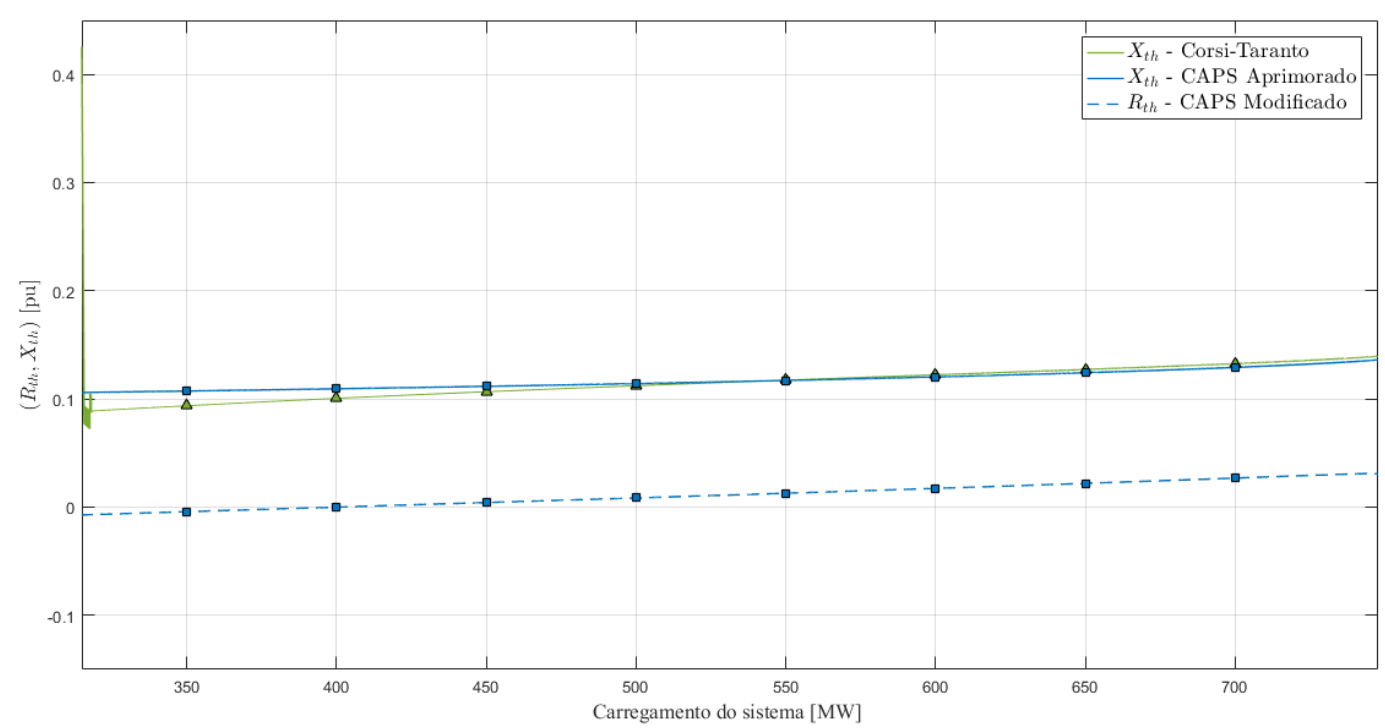

Figura 6.5: $R_{t h}$ e $X_{t h}$ em $[\mathrm{pu}]$ - caso 1

Ao fim da simulação calcula-se o erro relativo, mostrado na Tabela 6.8, em função dos módulos das impedâncias de carga e de Thévenin no ponto de MTP, assim definido como:

$$
\zeta_{r}[\%]=\left(\frac{\| Z_{L}|-| Z_{t h}||}{\left|Z_{L}\right|}\right)_{\mathrm{MTP}} \times 100
$$

Tabela 6.8: Erro de estimação para o caso 1 - Sistema WSCC 9 barras

\begin{tabular}{|ll|}
\hline Método & $\zeta_{r}[\%]$ \\
\hline \hline VIP & 0,57 \\
ŠVG & 0,57 \\
Corsi-Taranto & 1,27 \\
CAPS & 21,85 \\
CAPS Aprimorado & 1,48 \\
\hline
\end{tabular}

O valor estimado do módulo da tensão de Thévenin é apresentado na Figura 6.6, junto aos valores máximo e mínimo obtidos pelo método de CorsiTaranto, em cuja simulação foi implementada com parâmetro de atualização inicial $k=10^{-2}$ até a convergência, e posteriormente trocado para $10^{-4}$ para um ajuste refinado. A escolha de $k$, conforme mencionado, constitui um aspecto importante para o algoritmo, pois representa o parâmetro de correção efetivo, uma vez que, de um modo geral, $\dot{E}_{t h}$ apresenta relativo distanciamento entre os valores de $\dot{E}_{t h}^{\text {máx }}$ e $\dot{E}_{t h}^{\text {mín }}$. 


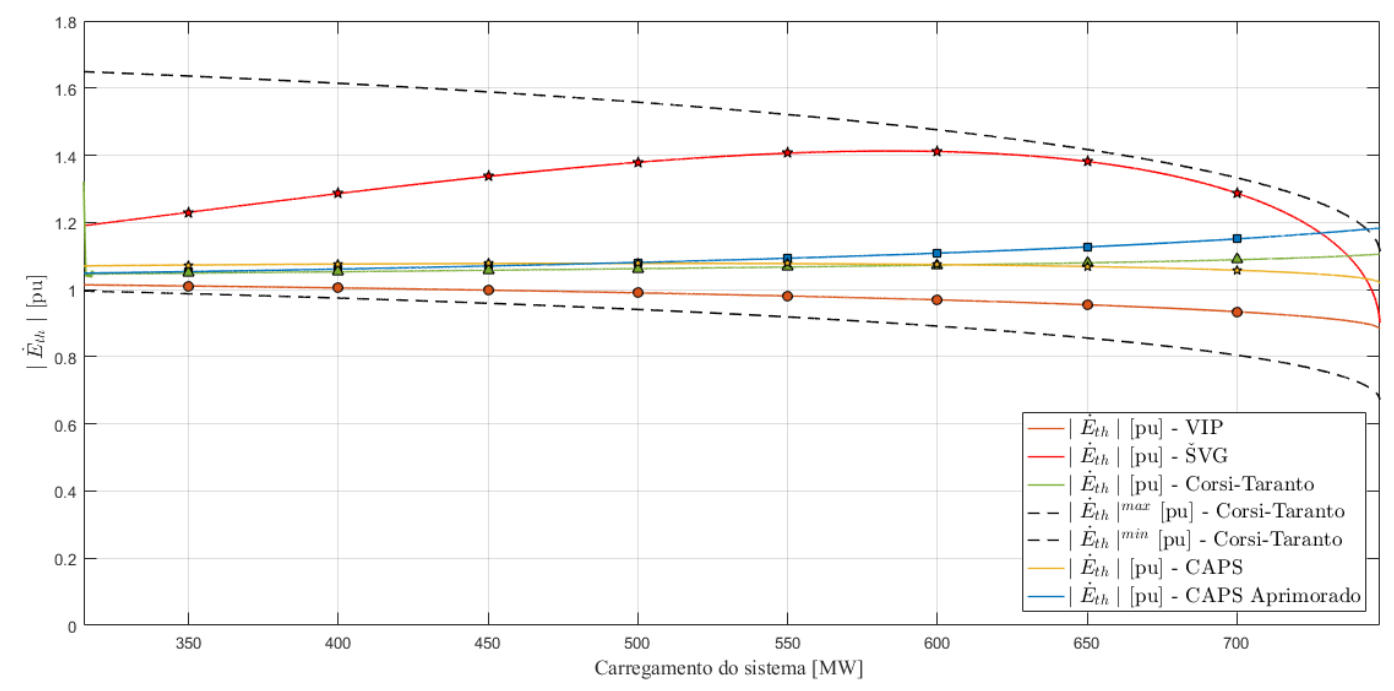

Figura 6.6: Gráfico de $\left|\dot{E}_{t h}\right|[\mathrm{pu}]$ - caso 1

Iterativamente, após a obtenção dos parâmetros do circuito equivalente em cada ponto de operação, estima-se a margem de carregamento da barra, que naturalmente deverá tender a zero na MTP, conforme mostrado na Figura 6.7. Através do valor de $S_{L}^{\text {máx }}$ obtido durante a simulação, é possível, através da equação (2.12), traçar a margem do sistema para estabelecer uma comparação de proximidade da margem de carregamento estimada ao valor real.

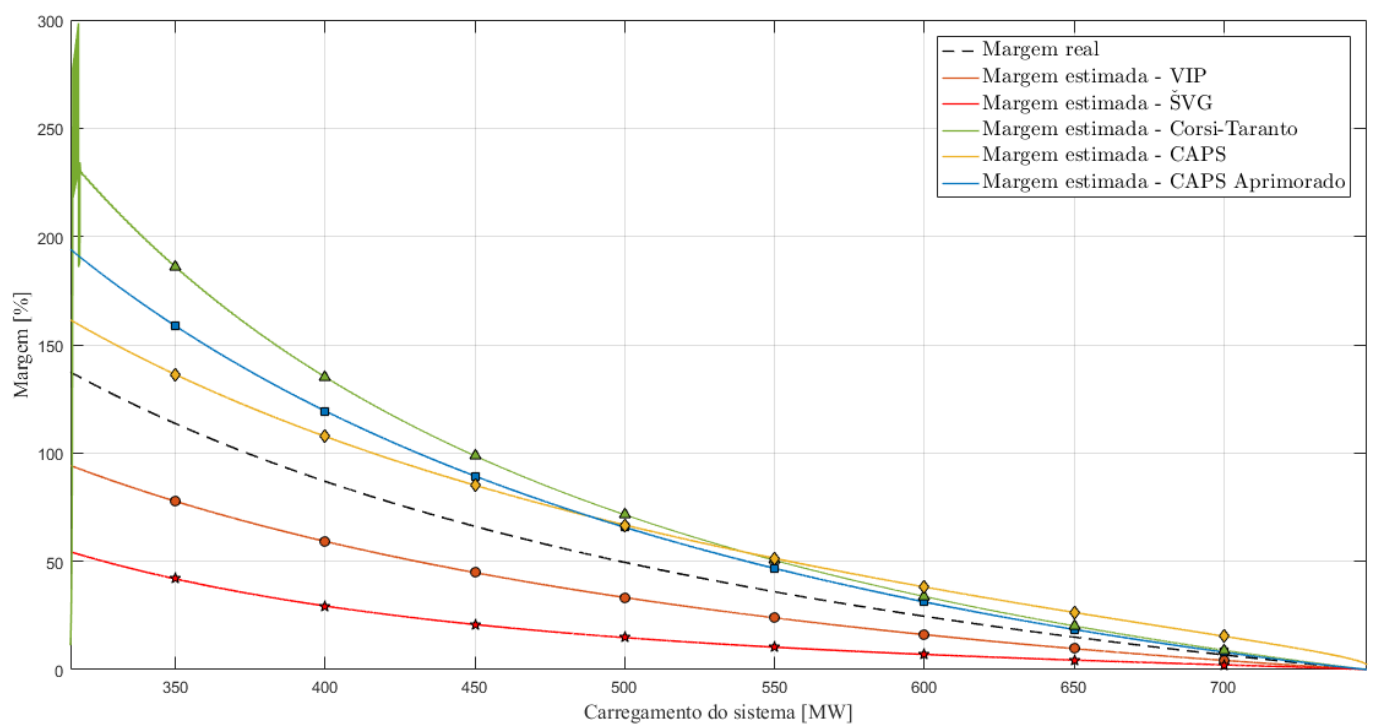

Figura 6.7: Margem de carregamento estimada [\%] - caso 1

Em [8], emprega-se o erro quadrático médio (EQM) para avaliar o desempenho dos métodos em cada caso, considerando a margem estimada. Deste modo, sendo a Margem estimada $_{j}$ o vetor correspondente à margem estimada em relação a barra $j$ das $N$ amostras e Margem $_{\text {real }_{j}}$ a margem real obtida pelo fluxo de carga, então: 


$$
\mathrm{EQM}=\frac{1}{N} \sum_{i}^{n}\left(\text { Margem }_{\text {real }_{j}}-\text { Margem }_{\text {estimada }_{j}}\right)^{2}
$$

Considerando então o conjunto de amostras que contemplam a convergência do método de Corsi-Taranto, chega-se aos valores apresentados na Tabela 6.9, em que se conclui que, embora o método CAPS seja incapaz de indicar o ponto de máximo carregamento, é o que melhor estima a margem do sistema.

Tabela 6.9: Erro médio quadrático associado à margem de carregamento estimada - caso 1

\begin{tabular}{|ll|}
\hline Método & EQM $\left(\times 10^{3}\right)$ \\
\hline \hline VIP & 0,382 \\
ŠVG & 1,601 \\
Corsi-Taranto & 1,256 \\
CAPS & 0,134 \\
CAPS Aprimorado & 0,525 \\
\hline
\end{tabular}

Para o caso 2, a simulação de um crescimento desproporcional não ofereceu dificuldades de convergência para o método de Corsi-Taranto, embora o número de amostras tenha sido drasticamente reduzidas de 13739 medições para 4153, e com exceção do método CAPS, cujas características serão analisadas em maiores detalhes na Seção 6.2.1, os modelos permitem detectar com aceitável precisão o máximo carregamento, como mostrado na Figura 6.8.

Constata-se pela Tabela 6.10 um aumento no erro relativo devido a estimativa da máxima transferência de potência, sendo o método de Corsi-Taranto o que apresenta maior prejuízo em seu desempenho com relação ao caso 1, que pode ser explicado pela redução no fator $X_{t h} / R_{t h}$ identificado pelo método CAPS aprimorado. 


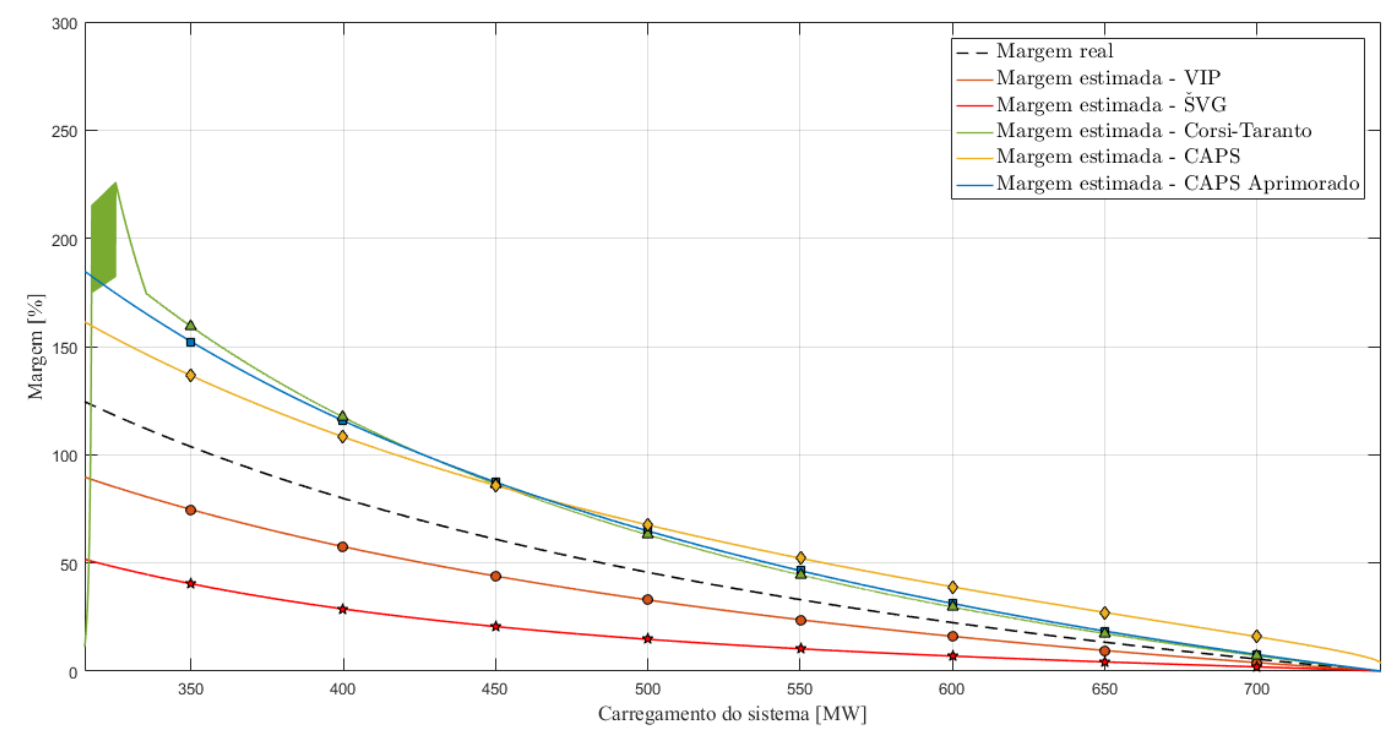

Figura 6.8: Margem de carregamento estimada [\%] - caso 2

Tabela 6.10: Erro de estimação para o caso 2 - Sistema WSCC 9 barras

\begin{tabular}{|ll|}
\hline Método & $\zeta_{r}[\%]$ \\
\hline \hline VIP & 1,00 \\
ŠVG & 1,00 \\
Corsi-Taranto & 3,15 \\
CAPS & 27,65 \\
CAPS Aprimorado & 2,65 \\
\hline
\end{tabular}

Novamente o modelo CAPS apresenta o menor EQM sendo, portanto, o que representa mais adequadamente a margem real da barra no caso 2, considerando o conjunto de amostras. Isto porque o método parte de uma margem estimada inicial mais próxima da margem real da barra 5, e a contribuição relativa ao resíduo quadrático inicial das primeiras amostras são menores, sendo estas parcelas responsáveis pelo maior impacto no EQM. Contudo, o método é incapaz de detectar o máximo carregamento, e para todos os pontos de operação analisados a margem é sobrestimada.

Tabela 6.11: Erro médio quadrático associado à margem estimada - caso 2

\begin{tabular}{|ll|}
\hline Método & EQM $\left(\times 10^{3}\right)$ \\
\hline \hline VIP & 0,214 \\
ŠVG & 1,126 \\
Corsi-Taranto & 0,604 \\
CAPS & 0,227 \\
CAPS Aprimorado & 0,546 \\
\hline
\end{tabular}




\subsection{1}

\section{Considerações sobre o Método CAPS}

Conforme descrito na Seção 5.4, o modelo CAPS compromete-se em representar explicitamente o efeito de acoplamento das cargas na barra em análise. Dentre as possibilidades na representação de tal efeito, foram estudados em [25] a adição de uma carga virtual $\dot{S}_{c j}$ na barra $j$, a inserção de uma fonte de tensão $\dot{E}_{c j}$ e a adição da impedância em série $\bar{Z}_{c j}$ ao circuito. A representação do efeito de acoplamento pela impedância mostrou-se promissora devido ao seu comportamento aproximadamente constante. Entretanto, tal premissa restringe-se a uma condição de aumento de carga proporcional, conforme observado em [26].

Para ilustrar o comportamento de $\bar{Z}_{c j}$ em condições desfavoráveis à premissa adotada por [25], impõe-se as seguintes direções de carregamento do sistema, apresentados na Tabela 6.12, para o sistema IEEE 14 barras em que se consideram os limites máximos e mínimos de potência reativa dos geradores nas barras 2, 3, 6 e 8, sendo interpretados pelo método através da variação súbita nas impedâncias mostrada nas Figuras 6.9 e 6.10. Obseva-se na Figura 6.9 que a impedância de acoplamento em todas as barras do sistema apresentam um perfil ligeiramente constante para o caso 1 , com crescimento de carga proporcional. No entanto, para o cenário de carga desproporcional apresentado na Figura $6.10, \bar{Z}_{c j}$ apresenta razoável variação que impacta na precisão da margem estimada, principalmente na detecção do máximo carregamento.

Tabela 6.12: Variações percentuais de carga no sistema IEEE 14 barras

\begin{tabular}{|cccc|}
\hline Caso & Barra & $\Delta P_{L}[\%]$ & $\Delta Q_{L}[\%]$ \\
\hline \hline 1 & $4,5,9,10,11,12,13,14$ & 0,01 & 0,01 \\
\hline \hline & 4,11 & 0,01 & 0,01 \\
2 & $5,10,12,14$ & 0,02 & 0,02 \\
& 9 & 0,03 & 0,03 \\
& 13 & 0,03 & 0,015 \\
\hline
\end{tabular}




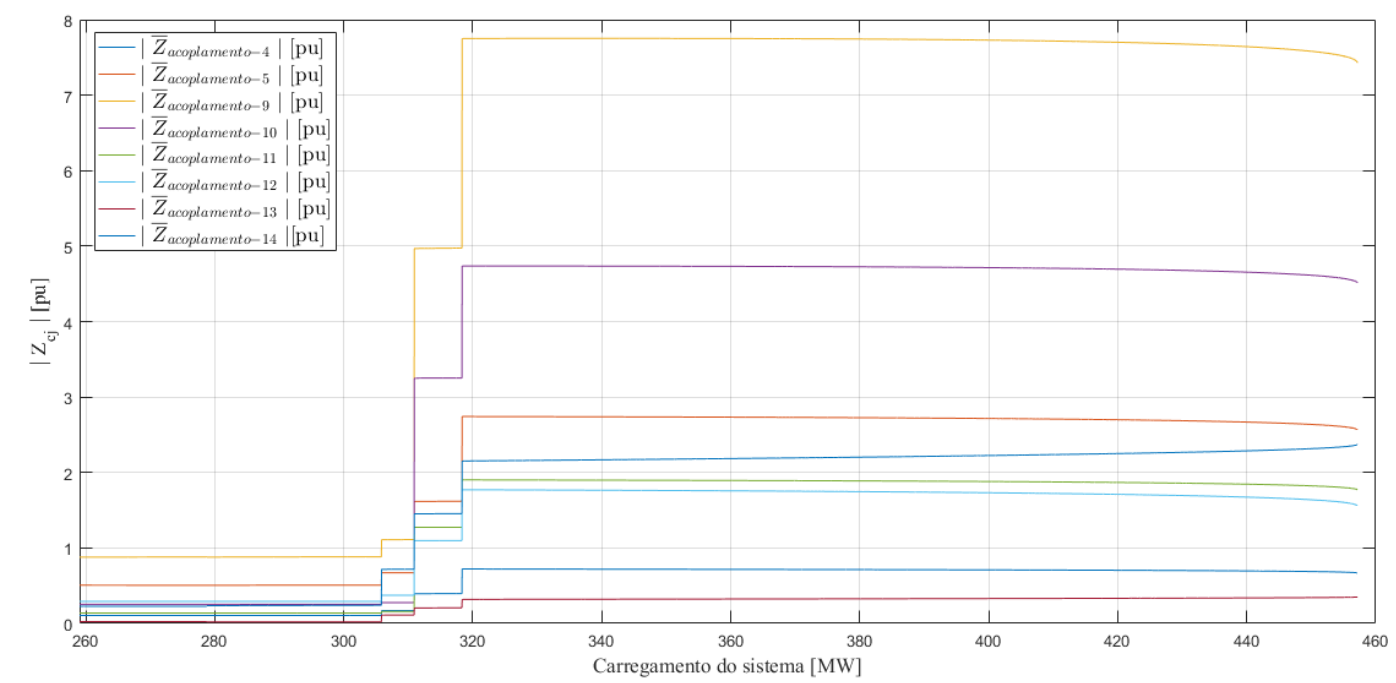

Figura 6.9: Comportamento de $\left|\bar{Z}_{c j}\right|[\mathrm{pu}]$ para crescimento de carga proporcional no sistema IEEE 14 barras

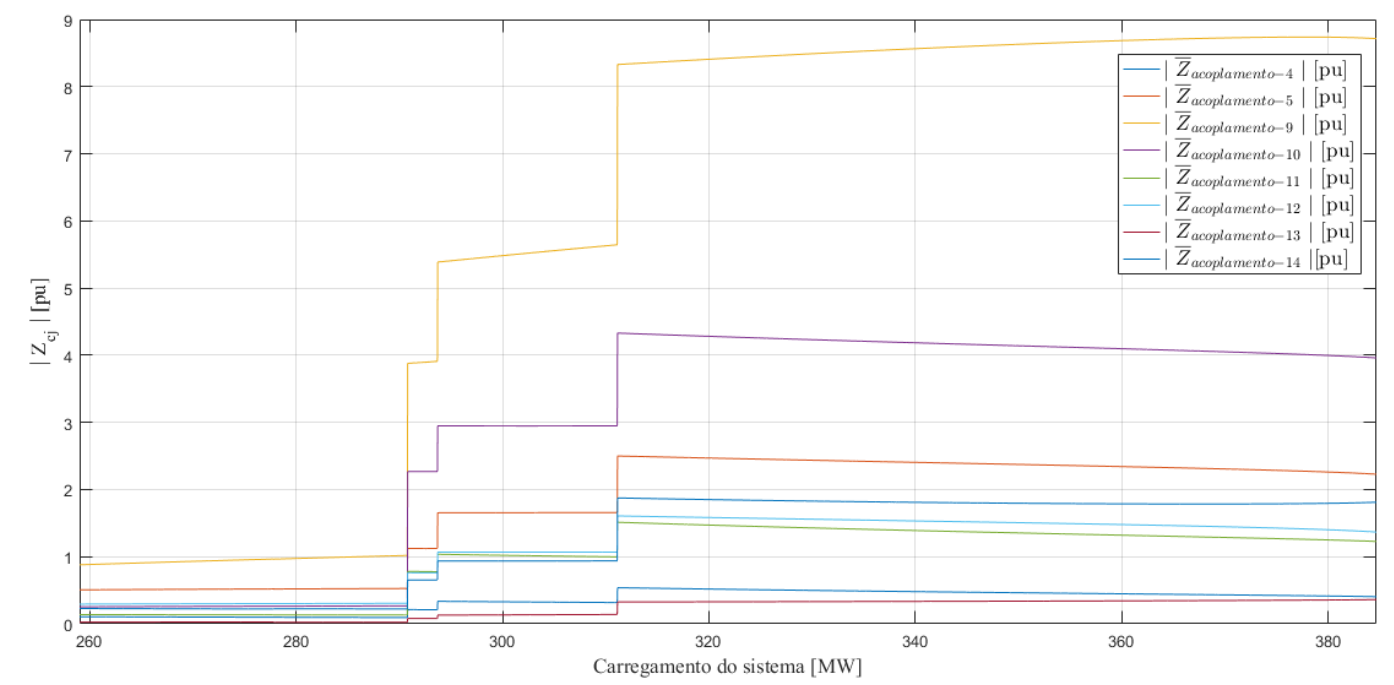

Figura 6.10: Comportamento de $\left|\bar{Z}_{c j}\right|[\mathrm{pu}]$ para crescimento de carga desproporcional no sistema IEEE 14 barras

Além das limitações do método em relação aos diferentes cenários de crescimento de carga, o modelo também enfrenta dificuldades dependendo da topologia da rede. Para ilustrar como as condições topológicas do sistema podem influenciar a margem estimada pelo método CAPS, emprega-se o sistema radial de 4 barras extraído de [27], em que as cargas $L_{1}$ e $L_{2}$ são isoladas pelos geradores $G_{1}$ e $G_{2}$, conforme mostrado na Figura 6.11. As linhas de transmissão são assumidamente idênticas, sendo nula as susceptâncias e resistências das linhas, e adotando as reatâncias iguais a 0,2 [pu]. Para as barras 2 e 4 alocam-se as cargas $L_{1}$ e $L_{2}$, respectivamente, com valores idênticos de $1,0+j 0,3[\mathrm{pu}]$. Nestas condições, apresentam-se então distintos cenários de 
despacho de potência ativa $P_{G_{2}}$ relativa ao gerador 2 , e que implica em uma potência devido ao gerador 1 de $P_{G_{1}}=2,0-P_{G_{2}}$ [pu]. Os dados do sistema considerando o caso base, onde $P_{G_{2}}=0,2[\mathrm{pu}]$, podem ser encontrados na Tabela 6.13 .

Tabela 6.13: Dados de barra - Sistema 4 barras - caso base

\begin{tabular}{|ccccccccc|}
\hline Barra & Tipo & $|\dot{V}|[\mathrm{pu}]$ & $\theta\left[{ }^{\circ}\right]$ & $P_{G}[\mathrm{pu}]$ & $Q_{G}[\mathrm{pu}]$ & $P_{L}[\mathrm{pu}]$ & $Q_{L}[\mathrm{pu}]$ & $b_{k}^{s h}[\mathrm{pu}]$ \\
\hline \hline 1 & $V \theta$ & 1,000 & 0,000 & 1,800 & 0,767 & & & \\
2 & $P Q$ & 0,920 & $-23,039$ & & & 1,000 & 0,300 & \\
3 & $P V$ & 1,000 & $-33,055$ & & 1,034 & & & \\
4 & $P Q$ & 0,910 & $-45,758$ & 0,200 & & 1,000 & 0,300 & \\
\hline \hline Total & & & & 2,000 & 1,801 & 2,000 & 0,600 & \\
\hline
\end{tabular}

A partir do caso base, varia-se a potência $P_{G_{2}}$ com incrementos $\Delta P_{G_{2}}=$ 0,2 [pu] até o limite de 2,0 [pu], e avaliam-se as condições de estabilidade de tensão do sistema pelos métodos CAPS e pelo fluxo de potência continuado. As margens individuais de $L_{1}$ e $L_{2}$ são obtidas pelo método CAPS, em que se verifica durante as simulações que a margem crítica está associada à $L_{2}$. Ademais, é possível concluir pela Figura 6.12, que para o despacho de potência no gerador 2 inferior a $0,6[\mathrm{pu}]$, os resultados dos métodos CAPS superestimam a margem do sistema, sendo para $P_{G_{2}}=0,2[\mathrm{pu}]$, a margem estimada de $L_{2}$ igual a 86, $01 \%$ em face dos 55,74\% do FPC. Nos cenários seguintes, a margem pelo método CAPS mantém-se fixa, e a margem do FPC aumenta para 75, 59\% em $P_{G_{2}}=0,4$ [pu], e se estabiliza em 85, 91\% nos demais cenários. Deste modo, é possível concluir que apenas para o despacho de potência ativa proveniente de $G_{2}$ na ordem de 0,6 [pu], os resultados do método CAPS são consistentes com aqueles obtidos pelo FPC.

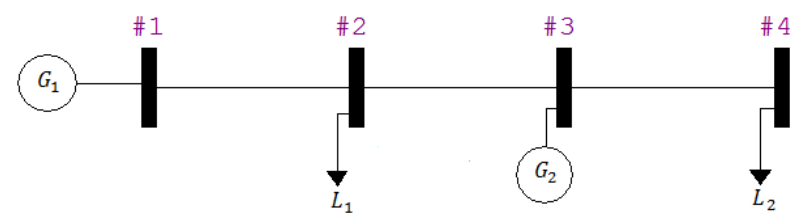

Figura 6.11: Sistema radial 4 barras

Deste modo, observa-se que quando a potência ativa injetada por $G_{2}$ é muito menor que $L_{2}$, a potência restante consumida pelo sistema deve ser atendida por $G_{1}$, e o fluxo de potência adicional requerido por $L_{2}$ restringe a capacidade de transmissão das linhas $1-2$ e $2-3$. Assim, a margem de $L_{1}$ é mínima, dentre os cenários simulados, quando $P_{G_{2}}$ é mínimo, pois exige-se ao máximo da capacidade de transmissão no trecho entre $G_{1}$ e $L_{1}$. Esta condição 
persiste no cenário seguinte, embora a margem de $L_{1}$ aumente de 242,91\% para 251,09\%, indicando uma redução no fluxo de potência no trecho devido a uma maior parcela de $L_{2}$ ser suprida por $G_{2}$. No entanto, ao ignorar o efeito de acoplamento que representa as limitações de carregamento da barra em análise, considerando as demais cargas do sistema, o método não consegue capturar as restrições impostas à margem de $L_{2}$, e produz resultados superestimados. De fato, isto se deve à matriz $\boldsymbol{Z}_{L L}$ que possui elementos nulos fora da diagonal principal, e que implica em uma impedância de acoplamento nula. O efeito de acoplamento só pode ser ignorado quando $G_{2}$ apresenta capacidade suficiente para atender $L_{2}$, aliviando assim o fluxo nos trechos entre as barras $1-2$ e $2-3$.

Conclui-se que através do rastreamento das impedâncias de carga e de Thévenin partindo do caso base, o método CAPS é incapaz de identificar a MTP. Conforme descrito no Capítulo 5, diversas modificações foram realizadas no método CAPS visando contornar a limitação do modelo. O método "Negative Load Model" (NL), impõe que o gerador $G_{2}$ seja substituído por uma barra de carga com injeção negativa, alterando a configuração de $\boldsymbol{Z}_{L L}$. O métodos CAPS Modificado, por sua vez, visa corrigir a impedância de Thévenin "vista" por cada barra através do parâmetro $\alpha_{j}$, calculado em cada medição.

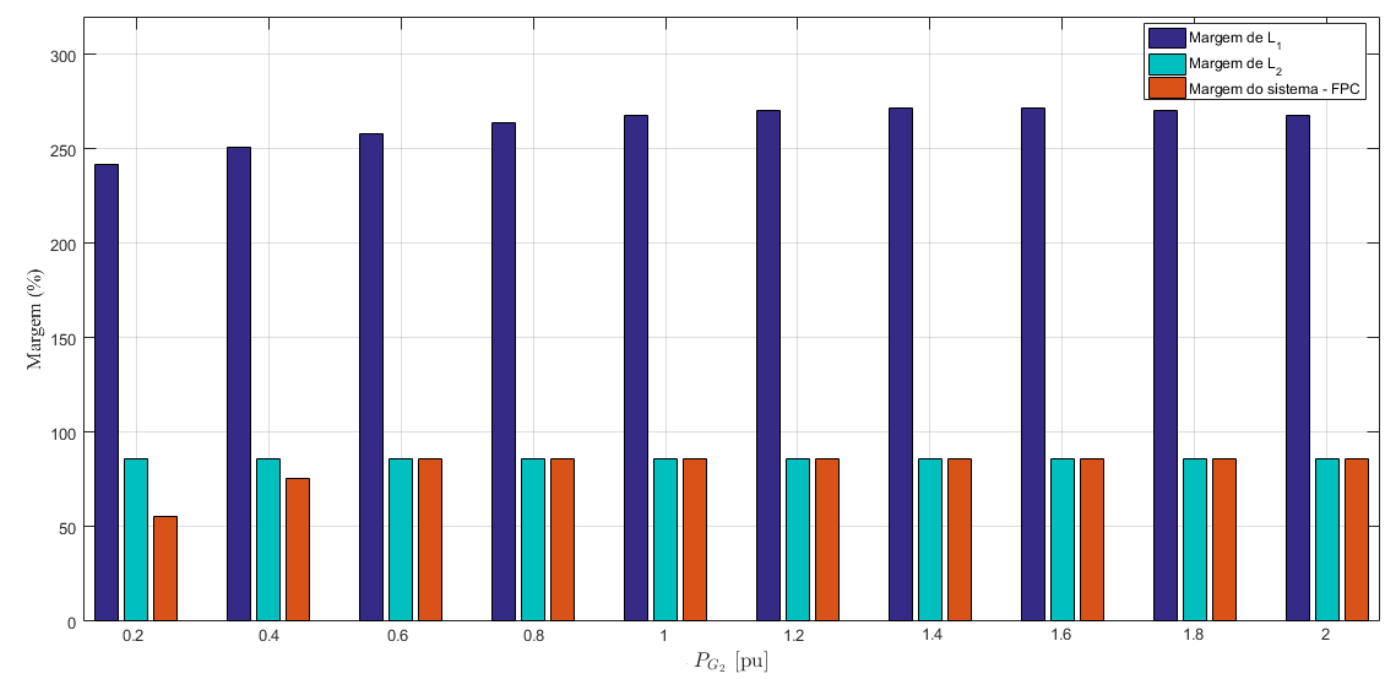

Figura 6.12: Margem individual de $L_{1}$ e $L_{2}$ estimadas pelo método CAPS

Com o gráfico de impedância em [pu] apresentado na Figura 6.13 e o erro associado ao casamento de impedância visto na Tabela 6.14, se observa que apenas os métodos CAPS Modificado e CAPS Aprimorado são capazes de prever a MTP corretamente. 


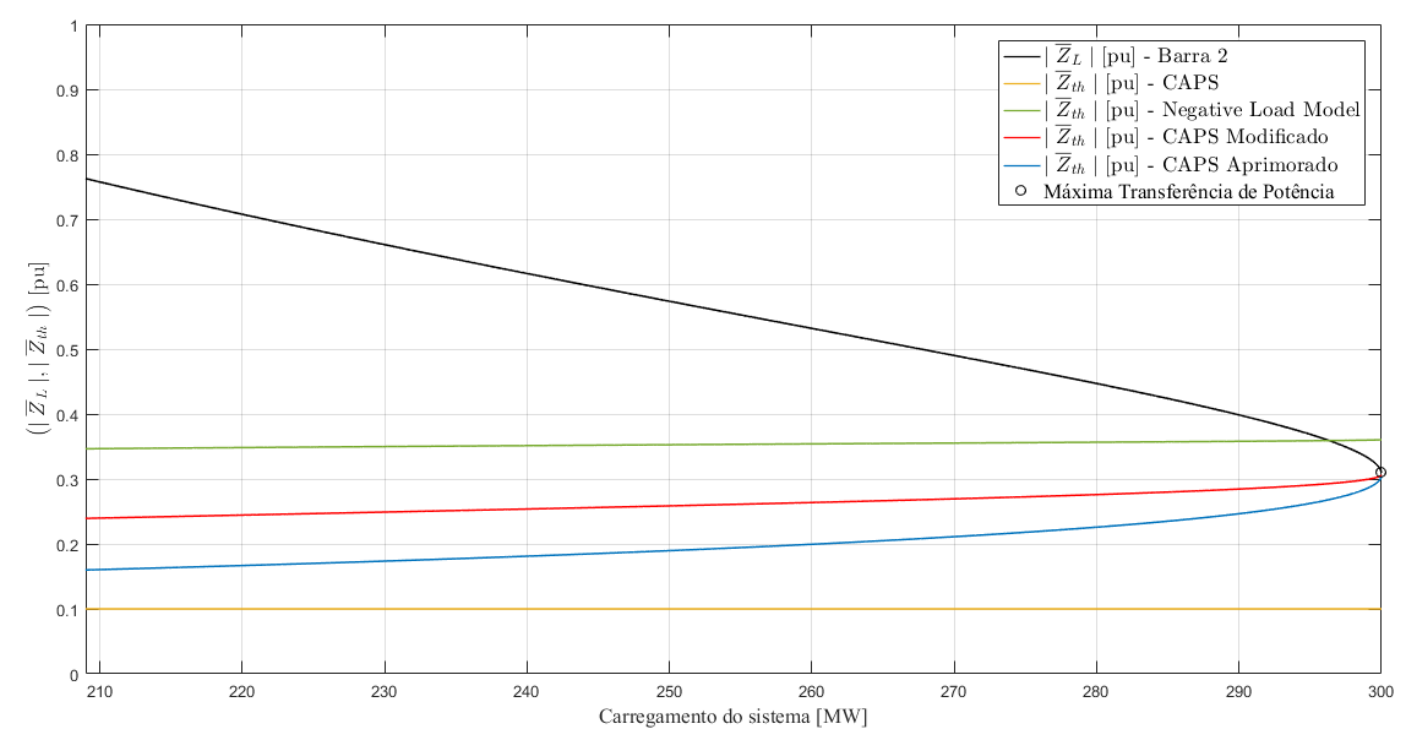

Figura 6.13: Gráfico de impedância em [pu] para o sistema 4 barras no caso base

Tabela 6.14: Erro de estimação para o caso base - Sistema 4 barras

\begin{tabular}{|lc|}
\hline \multicolumn{1}{|c}{ Método } & $\zeta_{r}(\%)$ \\
\hline \hline CAPS & 67,71 \\
Negative Loado Model & 16,40 \\
CAPS Modificado & 1,19 \\
CAPS Aprimorado & 2,13 \\
\hline
\end{tabular}

\section{2 .2}

\section{Considerações sobre os Métodos VIP, ŠVG e Corsi-Taranto}

Conforme mencionado anteriormente, diferentes contribuições acerca da comparação dos métodos de detecção da MTP foram publicadas, e a partir de então os aspectos que limitam os modelos passaram a ser conhecidos. Nesta seção serão discutidas algumas características dos métodos VIP, ŠVG e de Corsi-Taranto.

O método VIP foi concebido para condições em que a carga é alimentada radialmente pelo sistema [19], e um dos grandes obstáculos para aplicação do método reside na consideração do modelo de que, entre dois instantes consecutivos, os parâmetros do equivalente de Thévenin permanecem constantes. Esta premissa torna-se problemática quando implementada pois os eventos que ocorrem durante a dinâmica do sistema podem produzir pontos de singularidades no método. 
Neste sentido, o método ŠVG apresenta superioridade em relação ao método VIP, pois os pontos de singularidades podem ser evitados através do

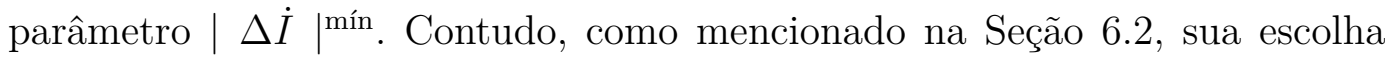
deve ser adequada, pois a impedância estimada pelo modelo pode não representar o equivalente "visto" pela barra em análise no ponto de operação atual. O efeito da influência da tolerância no método, ou sua sensibilidade à variação no patamar de carga, é apresentado na Figura 6.14 considerando o sistema WSCC 9 barras no caso 1, mostrado anteriormente, em que se demonstra a diferença na impedância de Thévenin estimada pelo modelo ŠVG tomando $|\Delta \dot{I}|^{\text {mín }}=10^{-5}$ e $|\Delta \dot{I}|^{\text {mín }}=0,001$. Observa-se que enquanto $\left|\dot{I}_{L_{5}}\right|<0,001, \mathrm{o}$ método continua estimando a impedância no ponto de operação inicial.

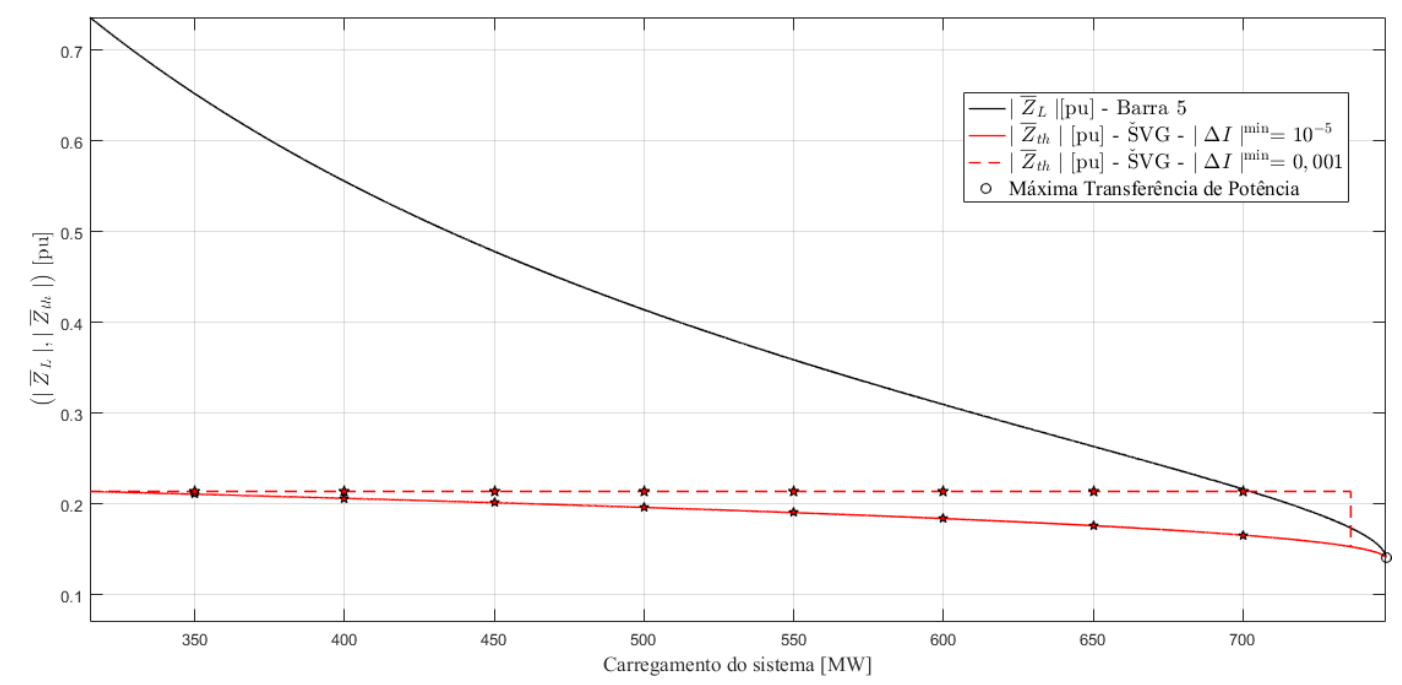

Figura 6.14: Influência de $|\Delta \dot{I}|^{\text {mín }}$ pelo método ŠVG através do gráfico de impedância em $[\mathrm{pu}]$

Adicionalmente, destaca-se que os modelos VIP e ŠVG são sensíveis à inserção de ruídos nas medições, exigindo, portanto, uma alta precisão nas amostras dos fasores de tensão e corrente.

O método de Corsi-Taranto, por sua vez, possui uma clara limitação quanto à aplicabilidade em redes de EAT. Sua exigência na alta taxa de medições, na ordem de 50 amostras por segundo, não representa um desafio de ordem prática pois as PMUs viabilizam as amostras em uma taxa de até 60 medições por segundo. Algumas recomendações sobre a escolha no valor do parâmetro $k$ são abordados em [23, 24], onde seu impacto na convergência do método é descrita em detalhes. Além disso, a possibilidade de um algoritmo auto-sintonizável baseado no fator de resposta da potência reativa é mencionada em [8], o que, segundo o autor, poderia conferir melhor desempenho quanto à velocidade de convergência do método. 


\section{3}

\section{Sensibilidade ao Ruído}

A avaliação da sensibilidade dos modelos ao ruído é inspirado nas referências [7, 8, 23, 24], e confere à análise um caráter mais realístico, uma vez que as imprecisões são inseridas intrisecamente nos fasores durante o processo de medição e transmissão de dados [8]. Tais imprecisões podem ser modeladas através da inserção de um ruído branco aos fasores de tensão e corrente, conforme mostrado na Figura 6.15 para a tensão medida na barra 5 considerando SNR ("signal-to-noise ratio") igual a $100 \mathrm{~dB}$.

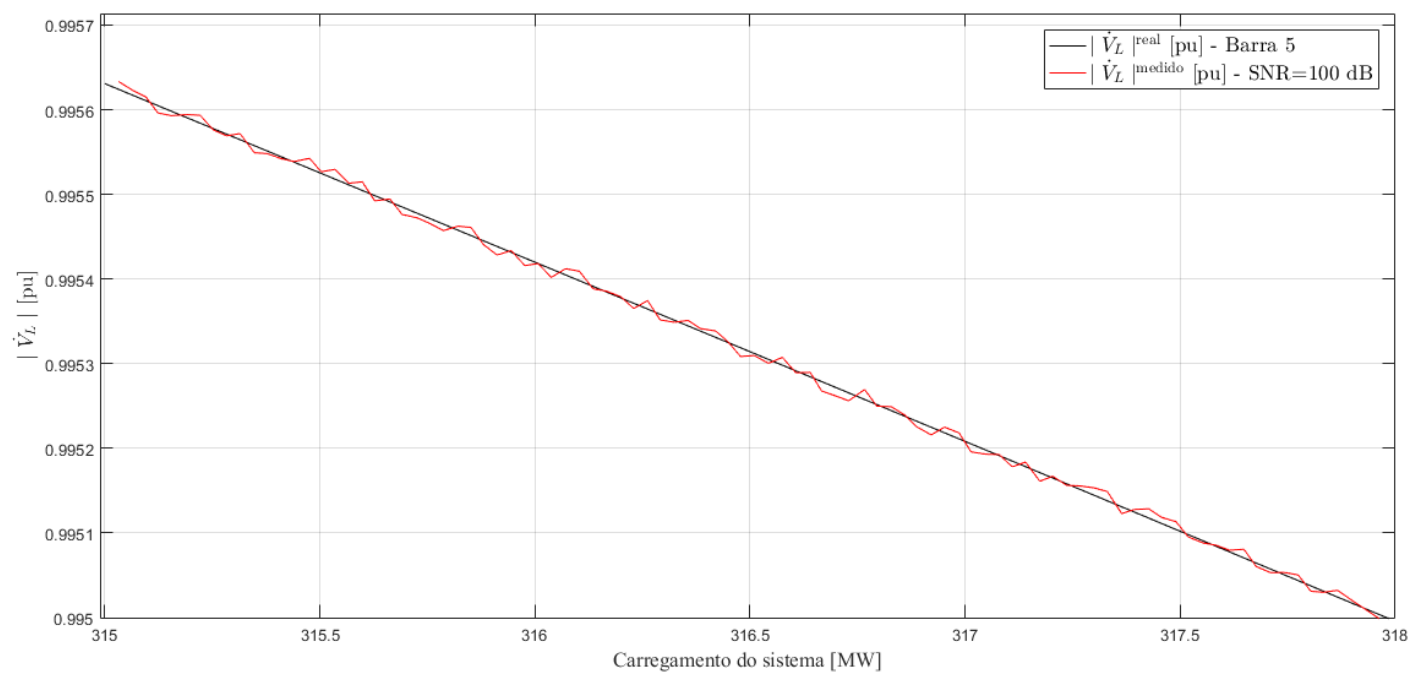

Figura 6.15: Tensão real $\left|\dot{V}_{L}\right|^{\text {real }}$ e tensão medida $\left|\dot{V}_{L}\right|^{\text {medida }}$ na barra 5

Para a simulação do método de Corsi-Taranto, foram inseridos ruídos nos fasores de tensão e corrente na barra 5 do sistema WSCC 9 barras analisado anteriormente considerando o caso 1 (com crescimento de carga proporcional em todas as barras) até a divergência do algoritmo, que ocorre para SNR igual a $80 \mathrm{~dB}$, como visto na Figura 6.16. 


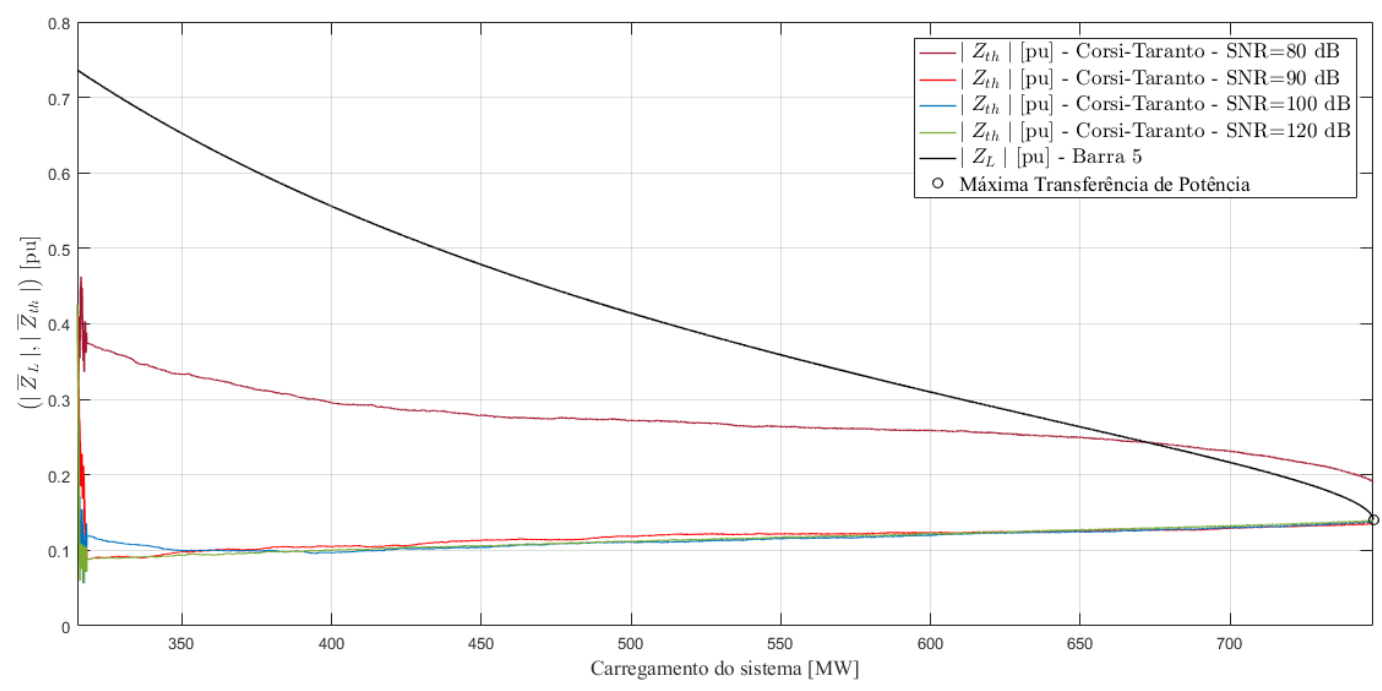

Figura 6.16: Resposta do método de Corsi-Taranto à presença de ruído

No método CAPS Aprimorado, as imprecisões foram adicionadas aos fasores de tensão e corrente na barra 5 e nas tensões dos geradores das barras 1,2 e 3. Como mostrado na Figura 6.17, o desempenho do algoritmo é fortemente influenciado pela introdução de ruídos, apresentando resultados consistentes apenas quando $\mathrm{SNR}=140 \mathrm{~dB}$. Isto se deve, em grande parte, ao parâmetro $\gamma$ que quantifica a resposta do sistema às variações de carga, e que utiliza medições consecutivas para estimá-la.

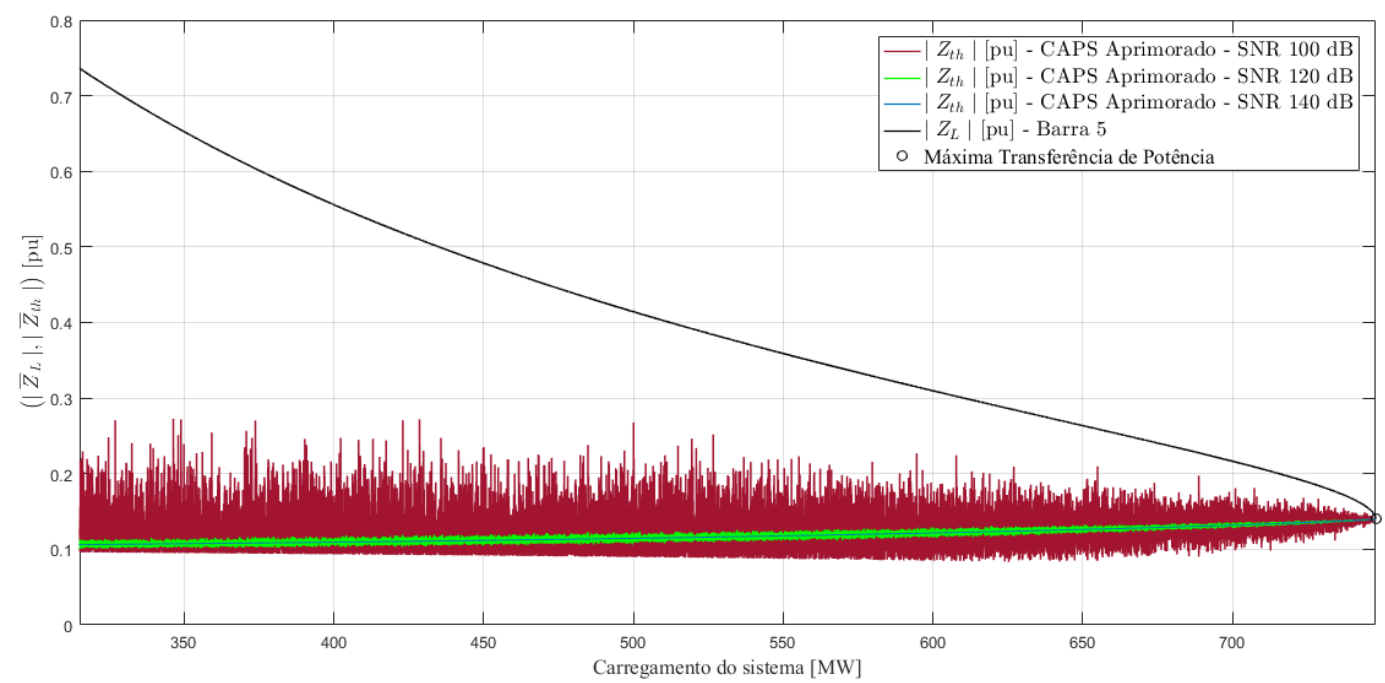

Figura 6.17: Resposta do método CAPS Aprimorado à presença de ruído

A Figura 6.18 ilustra o comportamento de $\gamma$ durante as simulações, sendo os valores de $\gamma$ para $\mathrm{SNR}=100 \mathrm{~dB}$ omitidos devido à grande variação na magnitude do parâmetro. 


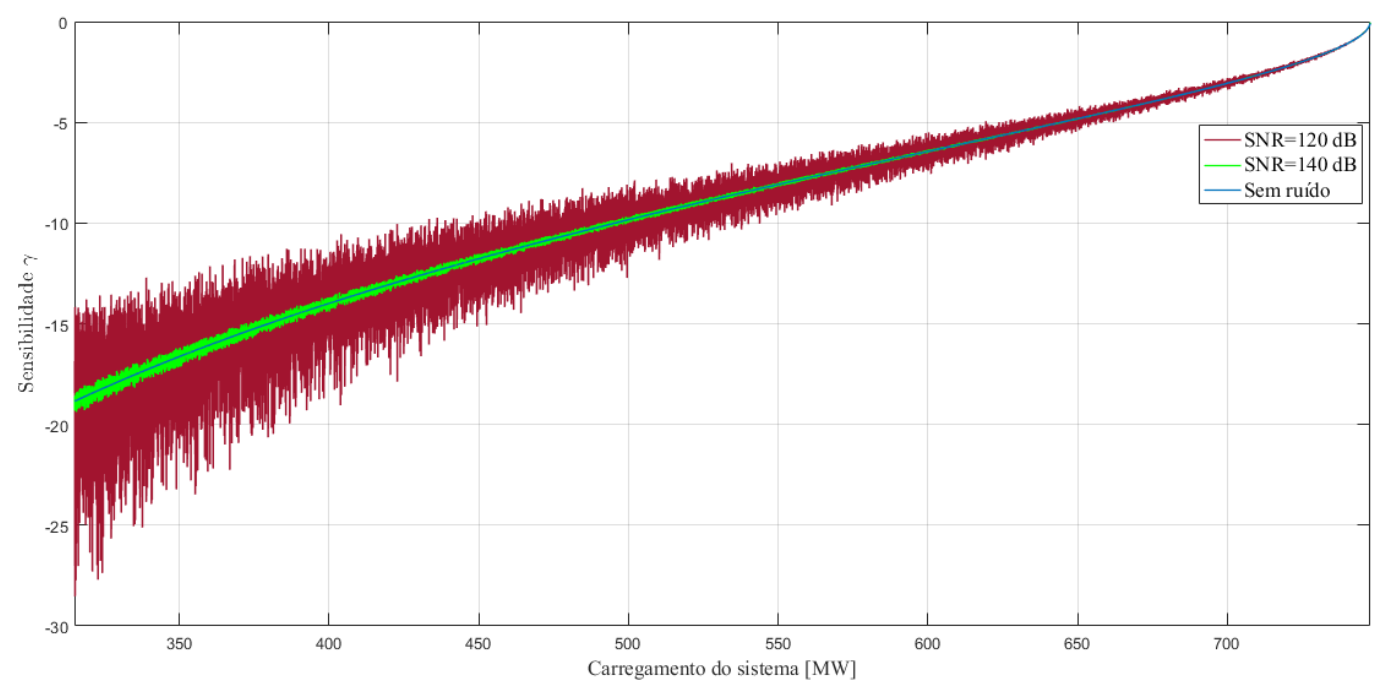

Figura 6.18: Resposta do fator de sensibilidade $\gamma$ à presença de ruído

\section{4}

\section{Avaliação das Barras de Transferência}

Neste seção, verifica-se a aplicação do método CAPS Aprimorado para as barras de transferência no sistema IEEE 30 barras, mostrado na Figura 6.19. Mantendo a coerência com as análises anteriores, avalia-se então as condições do sistema no ponto de operação inicial (caso base), em que os dados de barra são mostrados na Tabela 6.15, e consideram os limites de geração máxima e mínima de potência reativa dos geradores 2, 5, 8, 11 e 13, apresentados na Tabela B.5 do Apêndice B. No ponto de operação inical, o gerador da barra 2 atinge o limite máximo de $0,5[\mathrm{pu}]$ e a tensão deixa o seu valor especificado de 1,045 [pu] para atingir uma convergência em 1,043 [pu] como uma barra $P Q$.

Em seguida, os resultados provenientes do método da matriz $\boldsymbol{D}^{\prime}$ são apresentados na Tabela 6.16, em que se ocultou a coluna referente à ordenação devido à pouca relevância para este tipo de análise, e considerando que as barras de menores margens do sistema podem ser consultadas na Tabela 6.3. 


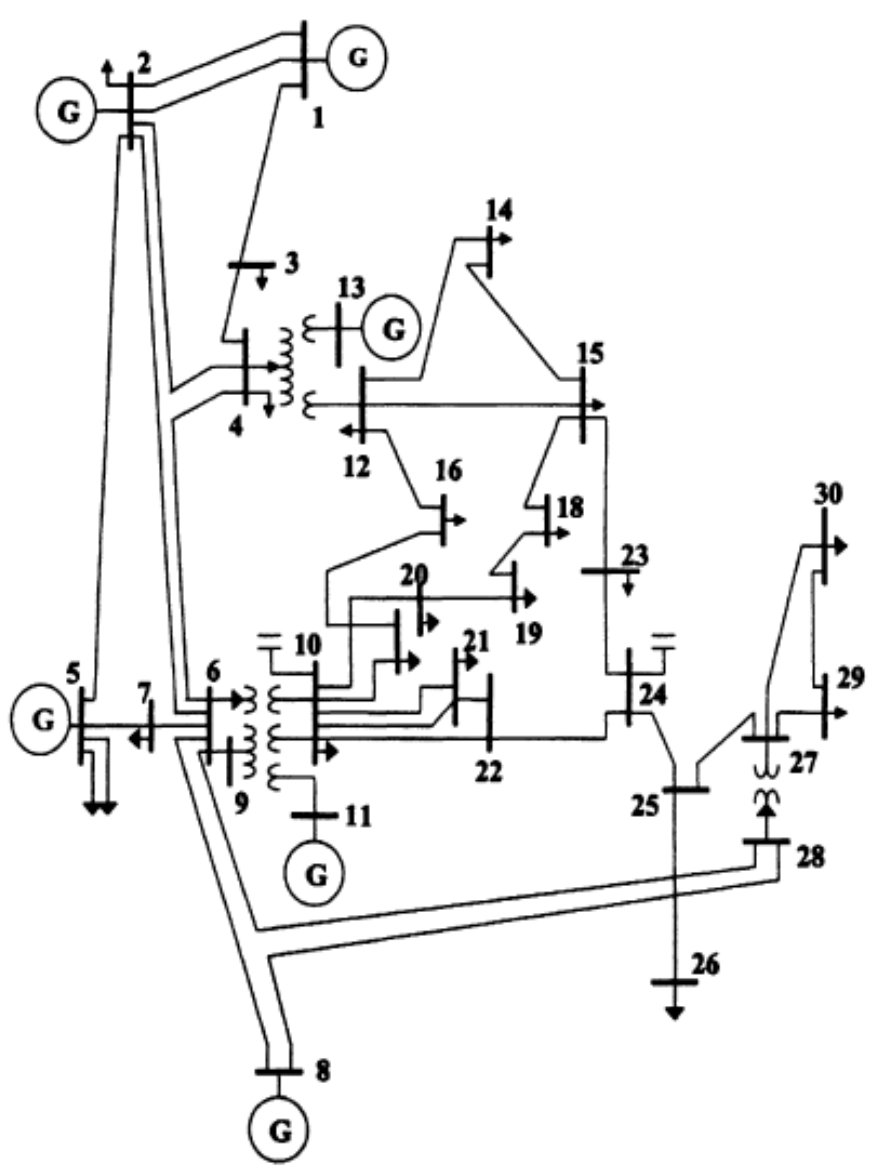

Figura 6.19: Sistema IEEE 30 barras

Durante a execução do fluxo de carga, avalia-se para a barra 27 a distribuição dos fluxos nos elementos a ela conectados, conforme mostrado na Figura 6.20. Para a aquisição dos pseudo-fasores utilizam-se sucessivos fluxos de potência considerando a rede íntegra com incremento de carga proporcional em todas as barras de 0,01 [\%], e gradativamente os geradores atingem os seus respectivos limites (na ordem: 2,8,5,11,13). Então, procede-se com a metodologia descrita na Seção 5.5.1 para análise das barras de transferência.

Sabendo que as seguintes alterações não modificam o ponto de operação do sistema original, adota-se uma carga fictícia igual à soma dos fluxos que deixam a barra 27, obtidos pela simulação em cada fluxo de carga anteriormente executado. Como a carga fictícia da barra 27 contempla os fluxos transferidos às barras adjacentes, as barras 29 e 30 são então suprimidas, e adiciona-se uma injeção equivalente ao fluxo que "entra" na barra 25. Como consequência, as conexões entre as barras $25-27,27-29,27-30$ e $29-30$ são eliminadas, e uma matriz de admitância nodal fictícia $\boldsymbol{Y}^{\text {fic }} \in \mathbb{C}^{28 \times 28}$ é pré-processada para dar início ao algoritmo CAPS Aprimorado. 
Tabela 6.15: Dados de barra - Sistema IEEE 30 barras

\begin{tabular}{|c|c|c|c|c|c|c|c|c|}
\hline Barra & Tipo & $|\dot{V}|[\mathrm{pu}]$ & $\theta\left[^{\circ}\right]$ & $P_{G}[\mathrm{pu}]$ & $Q_{G}[\mathrm{pu}]$ & $P_{L}[\mathrm{pu}]$ & $Q_{L}[\mathrm{pu}]$ & $b_{k}^{s h}[\mathrm{pu}]$ \\
\hline 1 & $V \theta$ & 1,060 & 0,000 & 2,610 & $-0,168$ & & & \\
\hline 2 & $P V$ & 1,043 & $-5,352$ & 0,400 & 0,500 & 0,217 & 0,127 & \\
\hline 3 & $P Q$ & 1,021 & $-7,532$ & & & 0,024 & 0,012 & \\
\hline 4 & $P Q$ & 1,012 & $-9,284$ & & & 0,076 & 0,016 & \\
\hline 5 & $P V$ & 1,010 & $-14,166$ & & 0,369 & 0,942 & 0,190 & \\
\hline 6 & $P Q$ & 1,010 & $-11,065$ & & & & & \\
\hline 7 & $P Q$ & 1,002 & $-12,865$ & & & 0,228 & 0,109 & \\
\hline 8 & $P V$ & 1,010 & $-11,813$ & & 0,371 & 0,300 & 0,300 & \\
\hline 9 & $P Q$ & 1,051 & $-14,109$ & & & & & \\
\hline 10 & $P Q$ & 1,045 & $-15,700$ & & & 0,058 & 0,020 & 0,190 \\
\hline 11 & $P V$ & 1,082 & $-14,109$ & & 0,162 & & & \\
\hline 12 & $P Q$ & 1,057 & $-14,943$ & & & 0,112 & 0,075 & \\
\hline 13 & $P V$ & 1,071 & $-14,943$ & & 0,106 & & & \\
\hline 14 & $P Q$ & 1,042 & $-15,836$ & & & 0,062 & 0,016 & \\
\hline 15 & $P Q$ & 1,038 & $-15,927$ & & & 0,082 & 0,025 & \\
\hline 16 & $P Q$ & 1,044 & $-15,526$ & & & 0,035 & 0,018 & \\
\hline 17 & $P Q$ & 1,040 & $-15,861$ & & & 0,090 & 0,058 & \\
\hline 18 & $P Q$ & 1,028 & $-16,542$ & & & 0,032 & 0,009 & \\
\hline 19 & $P Q$ & 1,026 & $-16,715$ & & & 0,095 & 0,034 & \\
\hline 20 & $P Q$ & 1,030 & $-16,519$ & & & 0,022 & 0,007 & \\
\hline 21 & $P Q$ & 1,033 & $-16,142$ & & & 0,175 & 0,112 & \\
\hline 22 & $P Q$ & 1,033 & $-16,128$ & & & & & \\
\hline 23 & $P Q$ & 1,027 & $-16,318$ & & & 0,032 & 0,016 & \\
\hline 24 & $P Q$ & 1,022 & $-16,495$ & & & 0,087 & 0,067 & 0,043 \\
\hline 25 & $P Q$ & 1,017 & $-16,067$ & & & & & \\
\hline 26 & $P Q$ & 1,000 & $-16,487$ & & & 0,035 & 0,023 & \\
\hline 27 & $P Q$ & 1,023 & $-15,542$ & & & & & \\
\hline 28 & $P Q$ & 1,007 & $-11,688$ & & & & & \\
\hline 29 & $P Q$ & 1,003 & $-16,772$ & & & 0,024 & 0,009 & \\
\hline 30 & $P Q$ & 0,992 & $-17,655$ & & & 0,106 & 0,019 & \\
\hline \multicolumn{4}{|l|}{ Total } & 3,010 & 1,340 & 2,834 & 1,262 & \\
\hline
\end{tabular}


Tabela 6.16: Método da Matriz $\boldsymbol{D}^{\prime}$ - Sistema IEEE 30 barras

\begin{tabular}{|ccccc|}
\hline Barra & $S_{i}[\mathrm{MVA}]$ & $S_{m}[\mathrm{MVA}]$ & Margem $[\%]$ & $\beta\left[^{\circ}\right]$ \\
\hline \hline $2^{\dagger}$ & 41,55 & 2607,54 & 98,41 & 91,26 \\
3 & 2,68 & 1434,02 & 99,81 & 91,04 \\
4 & 7,77 & 1738,12 & 99,55 & 90,28 \\
$5^{\dagger}$ & 95,88 & 716,04 & 86,61 & 100,95 \\
6 & 0,00 & 2169,80 & 100,00 & 93,57 \\
7 & 25,27 & 1084,45 & 97,67 & 97,01 \\
$8^{\dagger}$ & 30,84 & 910,92 & 96,61 & 93,88 \\
9 & 0,00 & 781,09 & 100,00 & 91,52 \\
10 & 6,14 & 669,41 & 99,08 & 93,42 \\
$11^{\dagger}$ & 16,17 & 297,54 & 94,56 & 92,18 \\
12 & 13,48 & 789,98 & 98,29 & 91,98 \\
$13^{\dagger}$ & 10,62 & 324,22 & 96,72 & 95,25 \\
14 & 6,40 & 366,98 & 98,26 & 94,28 \\
15 & 8,57 & 520,41 & 98,35 & 91,66 \\
16 & 3,94 & 446,02 & 99,12 & 91,03 \\
17 & 10,71 & 504,54 & 97,88 & 91,26 \\
18 & 3,32 & 339,55 & 99,02 & 90,39 \\
19 & 10,09 & 337,75 & 97,01 & 90,81 \\
20 & 2,31 & 361,03 & 99,36 & 90,58 \\
21 & 20,78 & 520,88 & 96,01 & 92,05 \\
22 & 0,00 & 517,36 & 100,00 & 91,64 \\
23 & 3,58 & 343,66 & 98,96 & 91,18 \\
24 & 10,98 & 387,15 & 97,16 & 90,61 \\
25 & 0,00 & 267,59 & 100,00 & 92,40 \\
26 & 4,19 & 119,60 & 96,50 & 92,79 \\
27 & 0,00 & 291,42 & 100,00 & 94,70 \\
28 & 0,00 & 1085,17 & 100,00 & 95,97 \\
29 & 2,56 & 145,99 & 98,24 & 95,33 \\
30 & 10,77 & 130,75 & 91,76 & 97,88 \\
\hline
\end{tabular}

$\dagger$ Barra de tensão controlada 

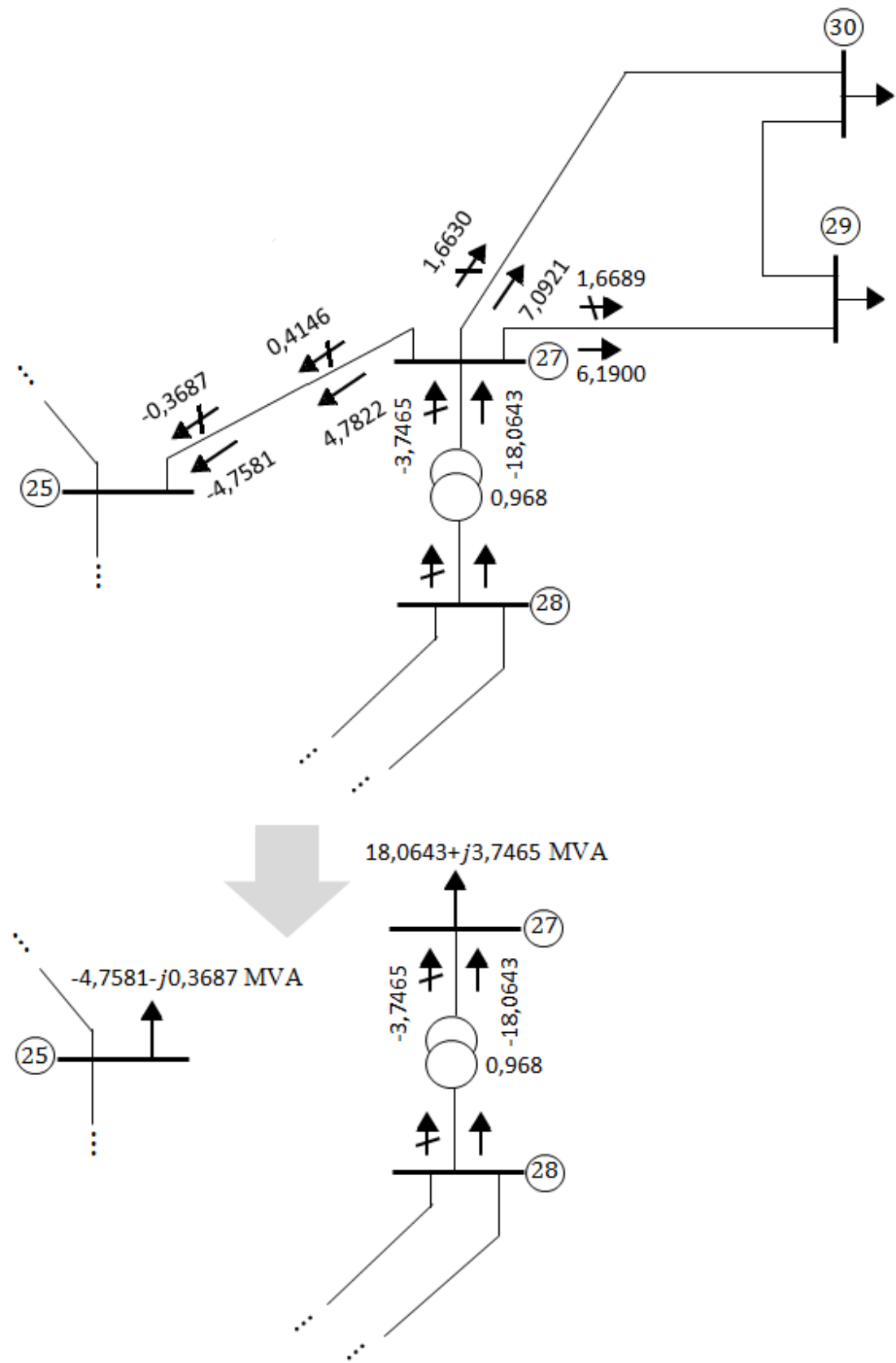

Figura 6.20: Distribuição dos fluxos em [MW] e [Mvar] nos elementos conectados à barra 27

No ambiente de simulação, é possível obter um índice associado a cada ponto de operação do sistema. Como se trata de uma barra de transferência, a margem calculada pelo método da matriz $\boldsymbol{D}^{\prime}$ mantém-se fixa em $100 \%$. Entretanto, a localização do ponto de operação em relação ao ponto crítico pode ser monitorado pelo índice $\beta$ da barra, em que $\beta$ representa a defasagem angular entre os vetores gradientes $\nabla \vec{P}_{i}$ e $\nabla \vec{Q}_{i}$. Como mencionado no Capítulo 4 , quando a barra em análise localiza-se na fronteira da curva $P V$, os vetores gradientes se encontram alinhados, o que implica em $\beta= \pm 180^{\circ}$ ou $\beta=0^{\circ}$.

$\mathrm{O}$ valor estimado da margem obtido pelo método CAPS Aprimorado para 
a barra de transferência 27 é visto na Figura 6.21, juntamente com o índice $\beta$. Com efeito, o alinhamento dos vetores gradiente não é atingido devido às características do fluxo de carga convencional, em que a matriz jacobiano torna-se singular nas proximidades do ponto crítico.

No entanto, como mostra a Figura 6.21, o valor de $\beta$ aproxima-se assintoticamente ao valor de $180^{\circ}$, que permite concluir que o modelo CAPS Aprimorado e o índice $\beta$ fornecido pelo método da matriz $\boldsymbol{D}^{\prime}$ estão em concordância ao identificar o ponto crítico.

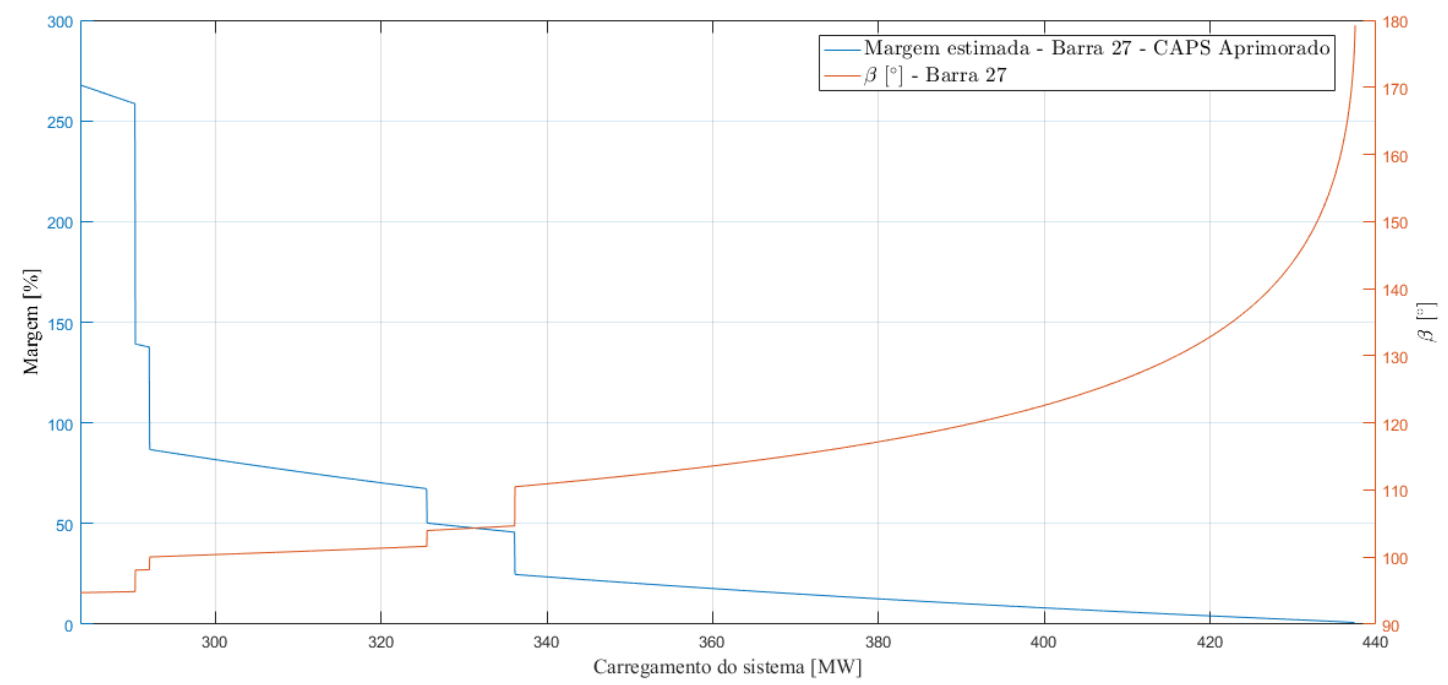

Figura 6.21: Rastreamento da margem de carregamento estimada pelo método CAPS Aprimorado e do ângulo $\beta$ obtido pelo método da matriz $\boldsymbol{D}^{\prime}$ para a barra 27 do sistema teste IEEE 30 barras

\section{5}

\section{Avaliação das Barras de Tensão Controlada}

Até este ponto procedeu-se com a análise estática para a avaliação das condições da margem de carregamento, levando o sistema à MTP através de sucessivos fluxos de carga. Nesta seção, a análise das barras de tensão controlada serão abordadas através da simulação dinâmica do sistema, com a representação dos reguladores de tensão (Automatic Voltage Regulator AVR), limitadores de sobre-excitação (Overexcitation Limiter - OXL) e dos reguladores de velocidade (Turbine Governor - TG).

Para a realização deste estudo foi utilizado o PSAT (Power System Analysis Toolbox), desenvolvido por Federico Milano como uma ferramenta integrada ao MATLAB, e sua escolha permite maior fluidez para a simulação devido a fácil acesso às variáveis de entrada para o modelo CAPS Aprimorado. Serão analisados 2 sistemas: o sistema teste radial de 3 barras e o modelo 
dinâmico do sistema WSCC 9 barras apresentado na Seção 6.2. A representação dos controles podem ser encontradas em [33] e os parâmetros utilizados localizam-se no Apêndice C.

\subsection{1}

\section{Sistema Teste Radial de 3 Barras}

\subsubsection{1}

\section{Caso 1 - Carga Pesada na Extremidade do Sistema Radial}

O sistema teste radial de 3 barras mostrado na Figura 6.22 possui duas linhas idênticas, e inicialmente com caso 1 foi alocada uma carga leve na barra 2 igual a 0,043 $+j 0,047[\mathrm{pu}]$, e uma carga pesada na barra 3 igual a $0,65+j 0,57[\mathrm{pu}]$. Para promover o aumento de carga no sistema, utiliza-se um arquivo de perturbação, adotando o crescimento proporcional de 0,1 [\%/s] nas parcelas ativa e reativa da carga, consideradas como do tipo potência constante. Desabilitou-se o limitador de sobre-excitação do gerador 1 e os parâmetros de TG encontrados no Apêndice $\mathrm{C}$ foram tais que garantissem ao gerador o constante suprimento de potência ativa ao sistema. Foram monitoradas as margens das barras 1 e 3 , isto porque a barra 3 representa a barra crítica que define a margem de carregamento sistema, conforme a equação 5.51.

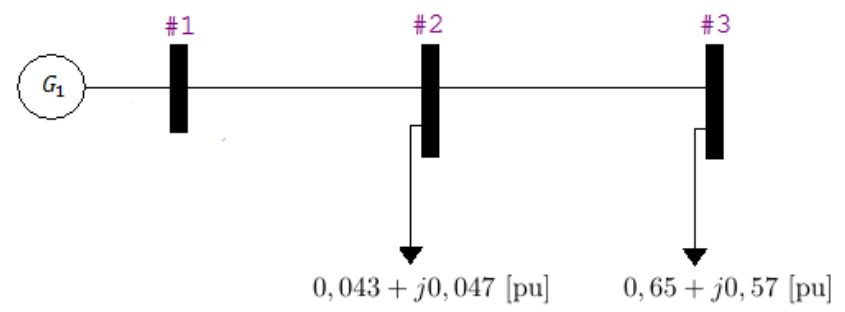

Figura 6.22: Sistema teste 3 barras - caso 1

Para o caso 1 observa-se no gráfico de impedância da Figura 6.23 que o máximo carregamento é identificado aos 842,7 segundos, quando a barra 3 apresenta uma margem nula. 


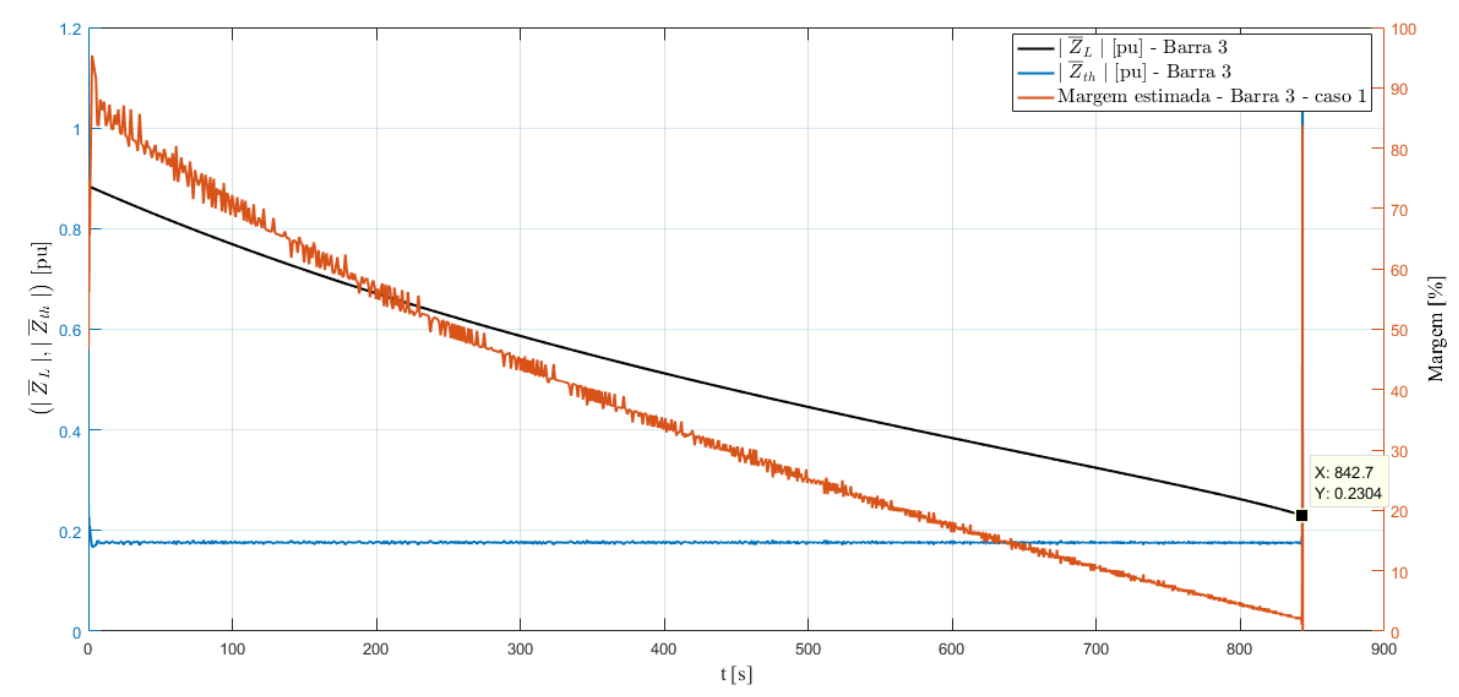

Figura 6.23: Gráfico de impedância em [pu] e margem de carregamento estimada em [\%] para a barra 3 - caso 1

Através da metodologia apresentada na Seção 5.5.2 utilizando o fator de sensibilidade $\psi$ obtém-se a margem estimada pela barra 1 , mostrado na Figura 6.24, em que é possível inferir que o gerador no instante identificado pelo casamento de impedância ainda apresentava margem de potência para injetar na rede. Este resultado permite concluir que a MTP não foi alcançada pela limitação do gerador, o que é condizente com a realidade do sistema pois o modelo do gerador pressupõe uma capacidade injeção muito além dos valores apresentados na simulação, como será visto no caso seguinte.

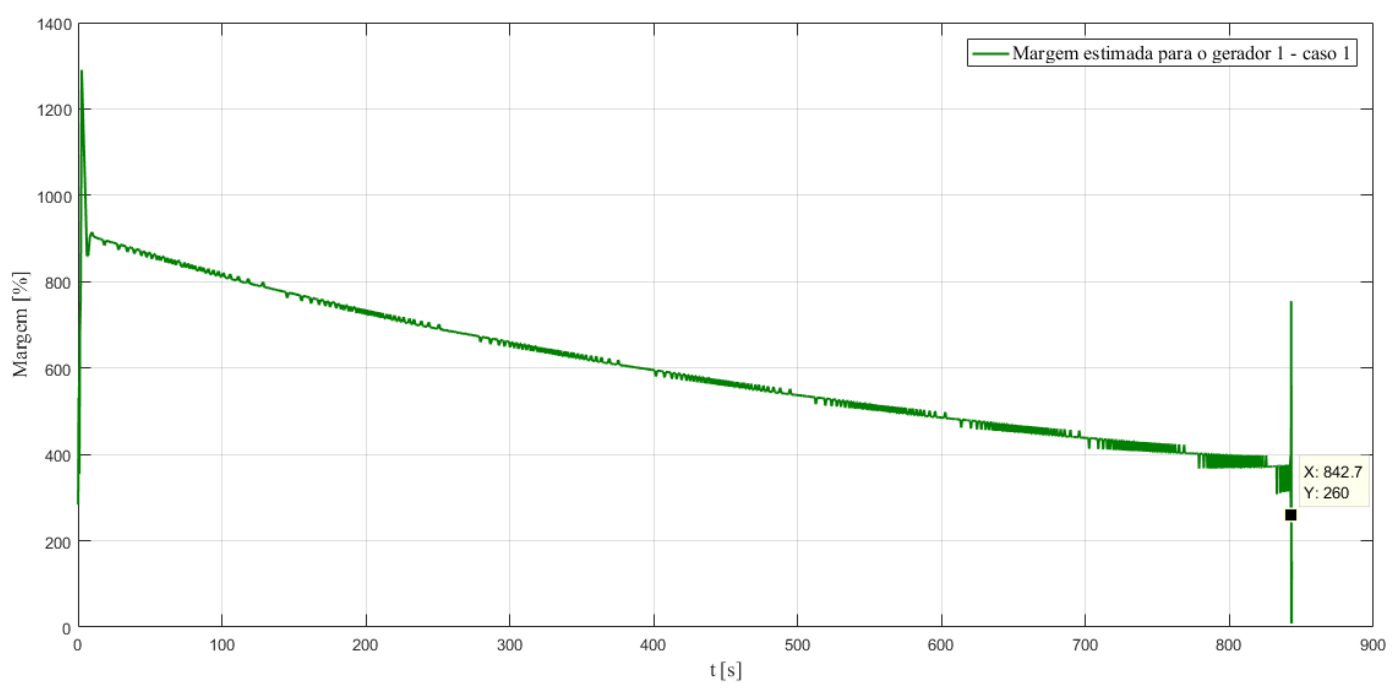

Figura 6.24: Margem estimada em [\%] para o gerador 1 - caso 1 


\subsubsection{2}

\section{Caso 2 - Carga Pesada na Barra Intermediária do Sistema Radial}

O caso 2 é ilustrado na Figura 6.25, e consiste da permutação entre as cargas, sendo então transferida para a barra 2 a carga de 0,65 + j0,57 [pu], e para a barra 3 a carga de 0,043+j0, 047 [pu]. Esta simples modificação permite antecipar uma conclusão baseado nos resultados do caso 1, isto é, sendo a carga leve praticamente desprezível no sistema, o esforço de transmissão do caso 1 entre o trecho $1-2$ é aproximadamente igual ao trecho $2-3$, pois o fluxo requerido pela carga leve é praticamente desprezível. Entretanto, para o caso 2 a carga pesada encontra-se na barra intermediária (barra 2), e neste caso o ramo crítico envolve a linha entre as barras $1-2$.

Portanto é razoável supor que sendo o caminho de transmissão crítico no caso 1 definido essencialmente pelo trecho $1-3$, e no caso 2 pelo trecho $1-2$, a impedância "vista" pela carga pesada seja maior no caso 1. Desta forma, a transferência de potência para o sistema, que basicamente depende do suprimento contínuo da carga pesada, é "facilitada" no caso 2. Espera-se então que a MTP atinja um patamar mais elevado e a margem de injeção pelo gerador 1 seja melhor explorada.

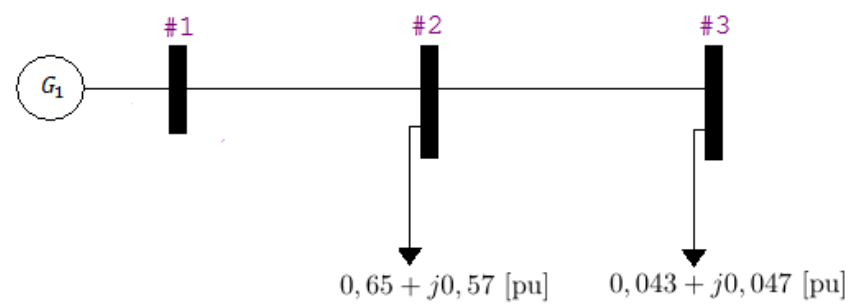

Figura 6.25: Sistema teste 3 barras - caso 2

Tendo em vista as considerações mencionadas, a simulação do caso 2 revela através da Figura 6.26 que a MTP ocorre em 2216 segundos. Neste instante o gerador 1 consegue injetar mais potência na rede, o que se traduz na redução da margem da barra 1 com relação ao caso anterior, e portanto a Figura 6.27 demonstra um resultado coerente com o esperado. 


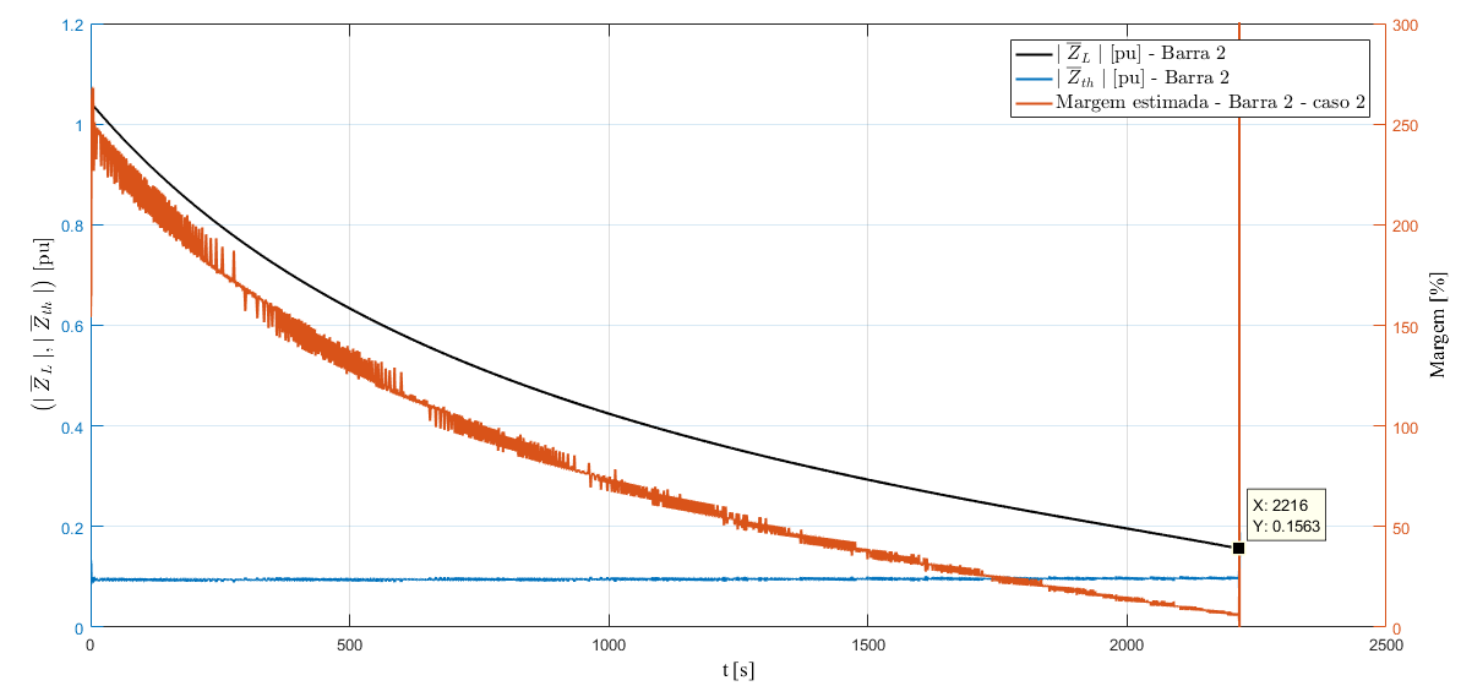

Figura 6.26: Gráfico de impedância em [pu] e margem de carregamento estimada em [\%] para a barra 3 - caso 2

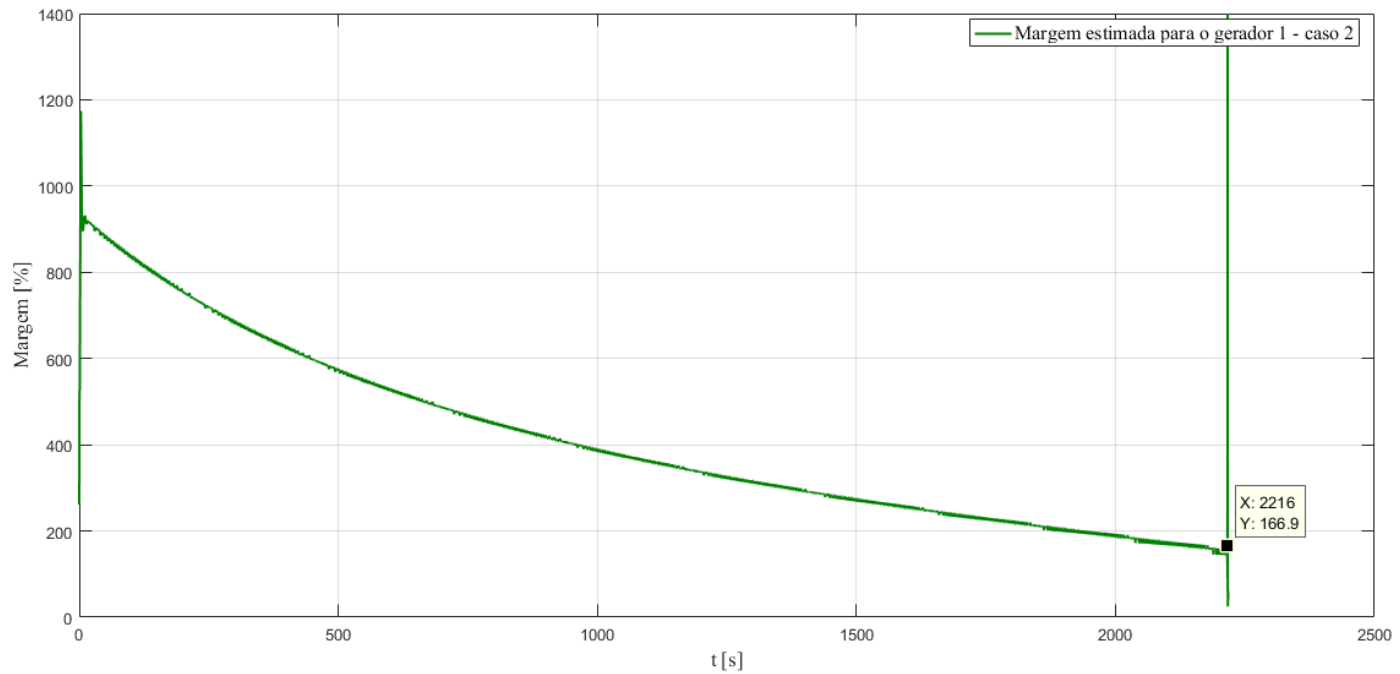

Figura 6.27: Margem estimada em [\%] para o gerador 1 - caso 2

\section{5 .2}

\section{Sistema Dinâmico WSCC 9 Barras}

\subsubsection{1}

\section{Caso 1 - Cenário sem OXL}

Os resultados obtidos no sistema teste de 3 barras mostraram-se promissores, o que conduz a uma investigação mais aprofundada do método, agora aplicado ao modelo dinâmico do sistema WSCC 9 barras mostrado na Figura 
6.28, cujo sistema encontra-se na base de exemplos do PSAT. Inicialmente, manteve-se desabilitado o OXL e o modelo do TG foi especificado para garantir a restrição de geração de potência ativa através dos limites da turbina. Isto permite avaliar como o método responde a este cenário de contingência.

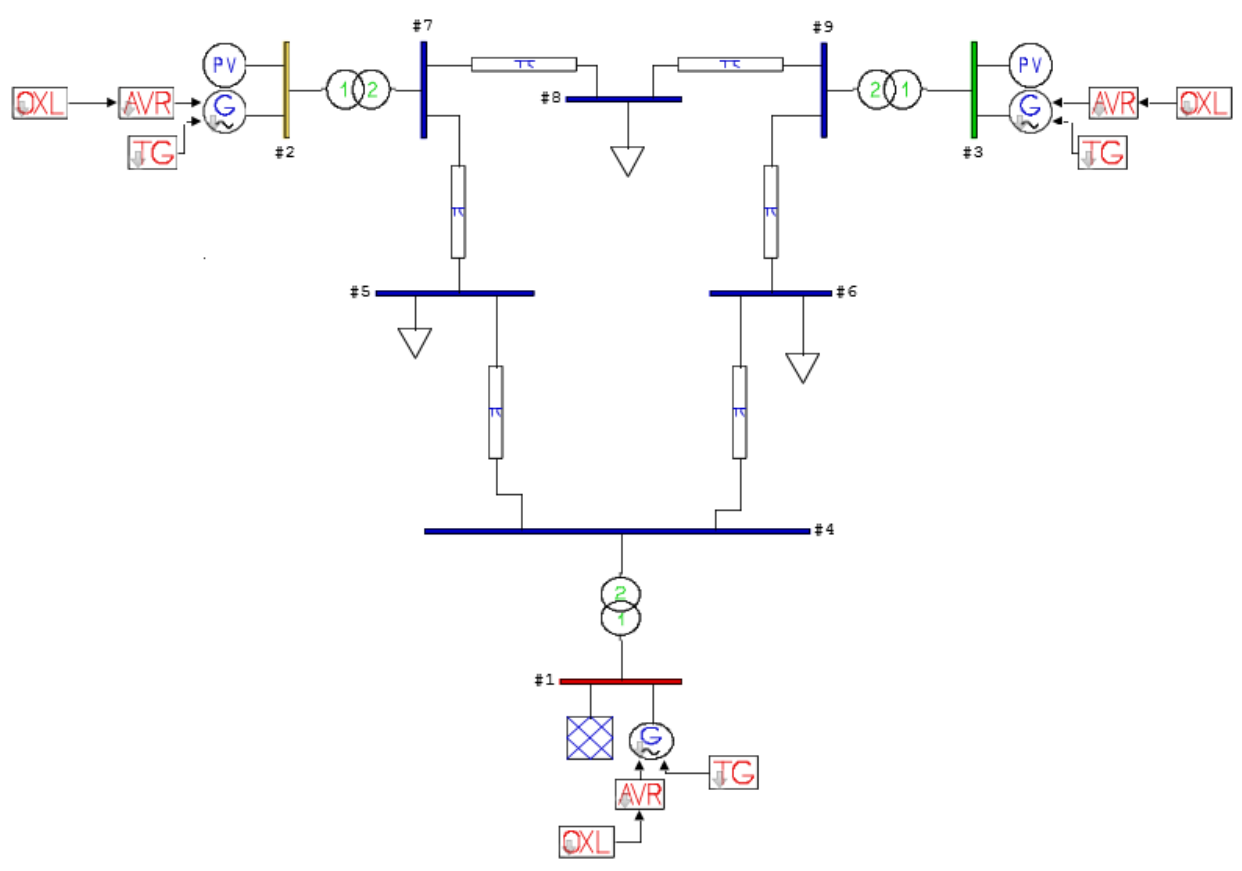

Figura 6.28: Modelo dinâmico do sistema WSCC 9 barras

Conforme descrito em [14], a existência da máxima carga a ser atendida pelo sistema enfrenta os seguintes fatores limitantes: o fluxo de potência que chega à barra de carga, o fluxo de potência injetado através da barra terminal do gerador, e o fluxo de potência que deixa a barra de geração e chega à barra de carga, simultaneamente. Deste modo, a identificação a respeito da manifestação da instabilidade de tensão lida com o monitoramento de um conjunto de barras. Os valores obtidos pelo método da matriz $\boldsymbol{D}^{\prime}$ obtido no ponto de operação inicial permite identificar a barra 5 como a barra de carga crítica, que será monitorada juntamente às barras de geração 2 e 3 .

Adotou-se, como no sistema anterior, um crescimento de carga proporcional de $0,1[\% / \mathrm{s}]$ nas componentes ativa e reativa das cargas do sistema, cujo comportamento do módulo da tensão nas barras são apresentados na Figura 6.29. Observa uma oscilação de tensão nos instantes finais que se deve à perda de sincronismo das máquinas. 


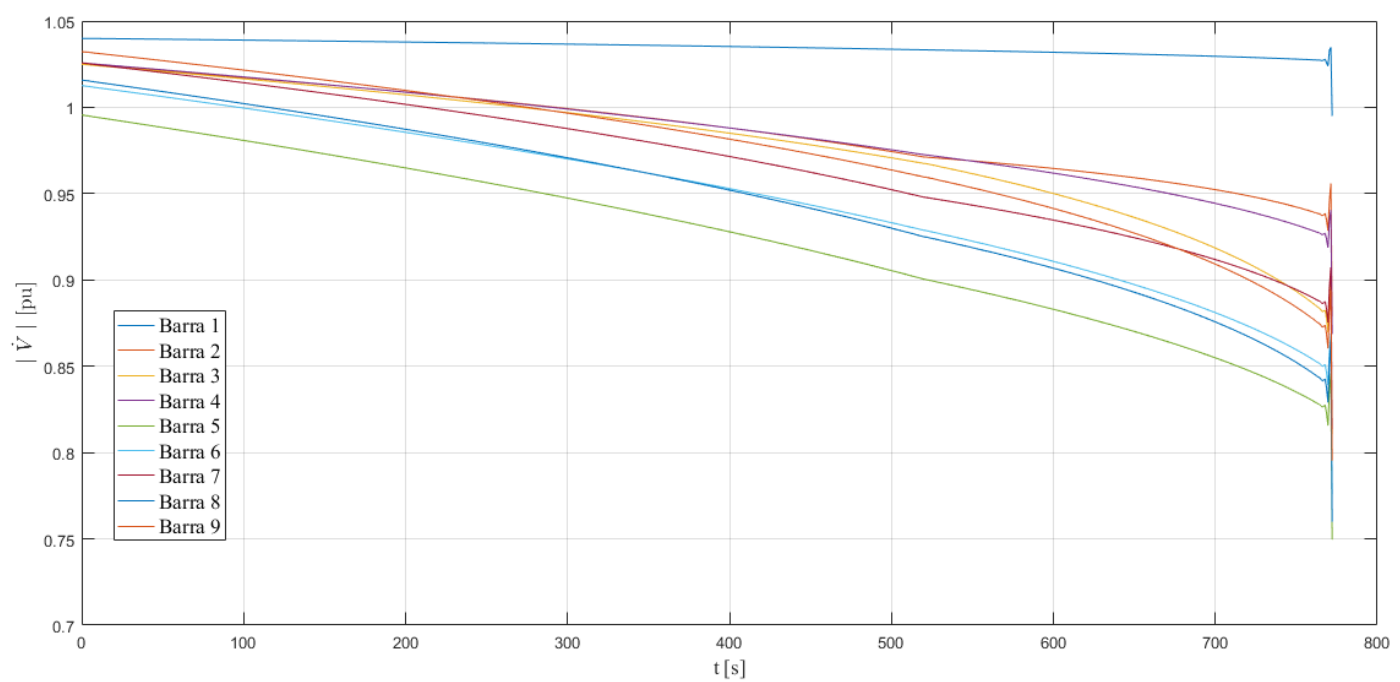

Figura 6.29: Gráfico do módulo da tensão em [pu] - caso 1

Com o limite da tubina alcançado pelo gerador 2 em aproximadamente 519,6 segundos e identificado na Figura 6.30, o gráfico de impedância da barra 5 reflete o evento através uma perturbação no valor estimado da impedância de Thévenin. Nota-se que a margem estimada pelos geradores, mostrada na Figura 6.31, indica que nos instantes iniciais a barra 2 apresenta uma margem inferior à barra 3 , portanto, em concordância com a avaliação pelo método da matriz $\boldsymbol{D}^{\prime}$. Após o evento de contingência, a margem da barra 3 torna-se menor. Com efeito, o gerador 3 passa a assumir maior responsabilidade no suprimento de carga com relação aos instantes anteriores, afirmação que é corroborada pela Figura 6.30.

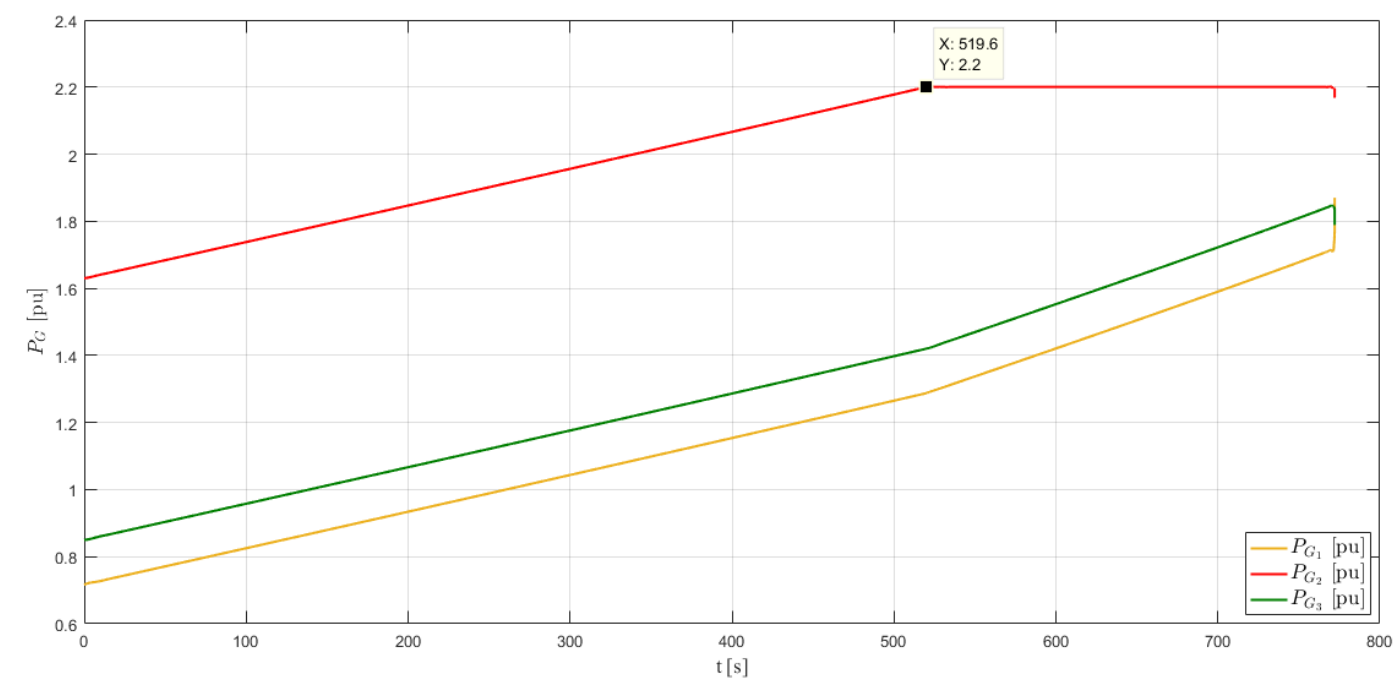

Figura 6.30: Potência ativa gerada em [pu] pelos geradores 2 e 3 - caso 1 


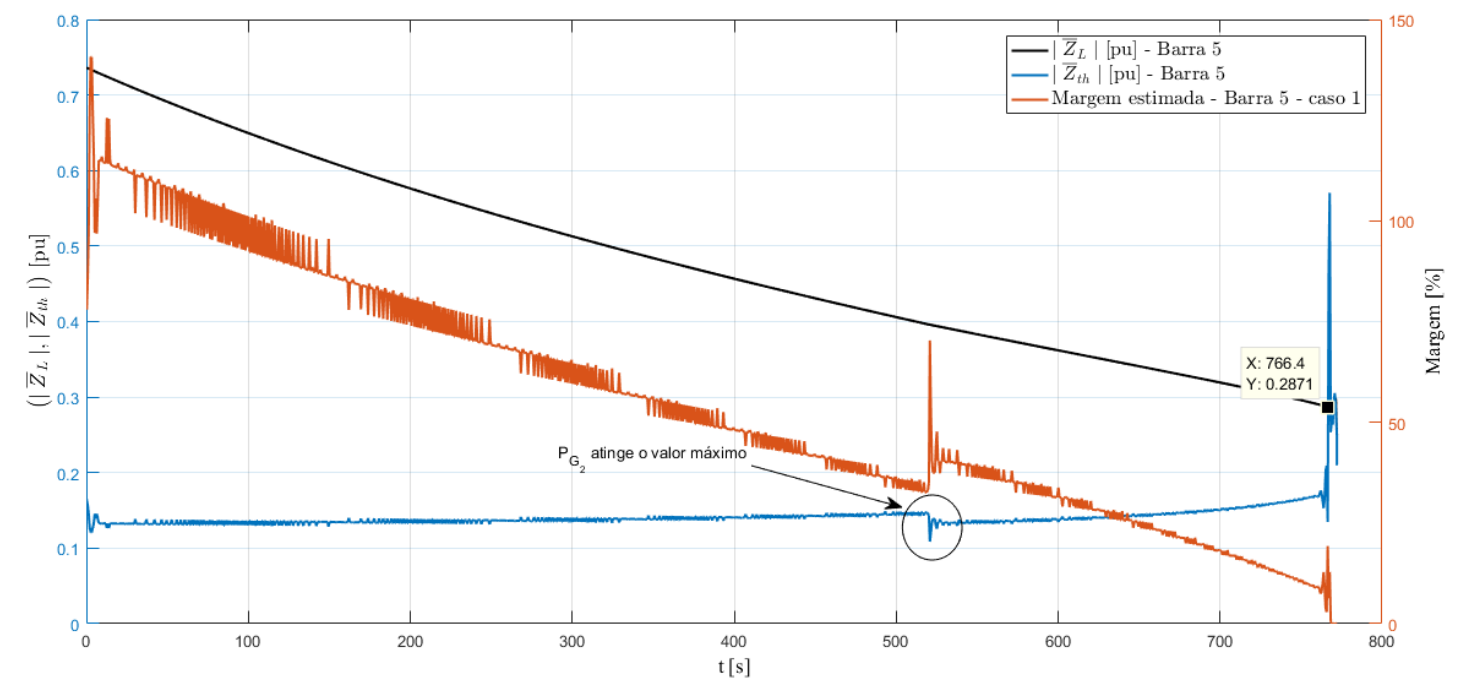

Figura 6.31: Gráfico de impedância em [pu] e margem de carregamento estimada em [\%] para a barra 5 - caso 1

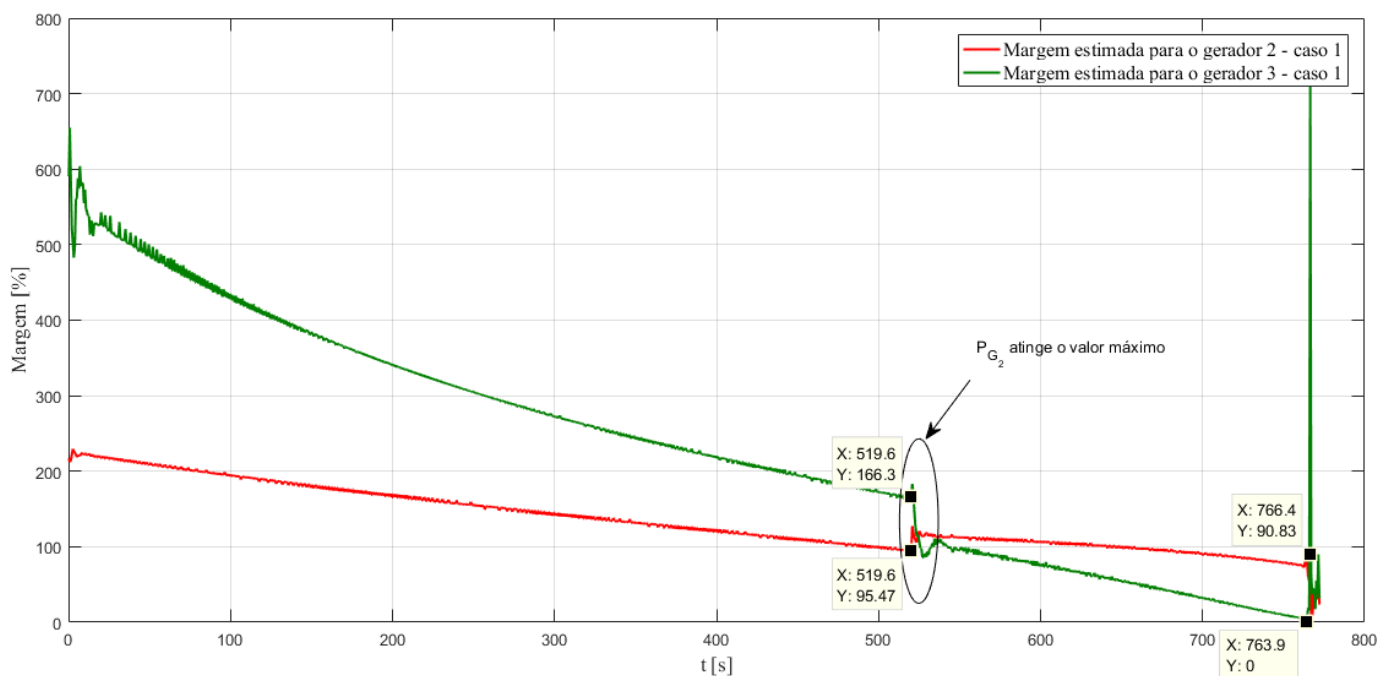

Figura 6.32: Margem estimada em [\%] para os geradores 2 e 3 - caso 1

A respeito da MTP, sua identificação pelo gráfico de impedância da Figura 6.31 ocorre aos 766,4 segundos, quando não há mais margem de carregamento para a barra 5 . Neste instante, o gerador 2 encontra-se com uma margem de 90,43\%, enquanto que o gerador 3 apresenta uma margem esgotada aos 763,9 segundo, portanto, 2,5 segundos antes do casamento de impedância. Deste modo, o método detecta a iminente instabilidade de tensão provocada pela máxima injeção de potência na rede, quando a potência demandada pelo sistema iguala-se à máxima potência estimada para o gerador. 


\subsubsection{2}

\section{Caso 2 - Cenário com OXL}

No caso 2 considera-se o OXL para avaliar seu impacto no modelo proposto, tendo em vista a avaliação das barras de geração. O OXL tem como objetivo a proteção dos enrolamentos de campo do gerador contra o sobreaquecimento causado quando estes são submetidos a condições prolongadas de sobrecorrente [32].

Neste caso, será empregado novamente o método de Corsi-Taranto para se estabelecer uma comparação direta sobre a resposta dos modelos em relação à detecção da MTP, uma vez que o método de Corsi-Taranto apresenta bom desempenho nestas circunstâncias. O sistema foi submetido a um percentual de carregamento idêntico aos casos anteriores, de $0,1 \%$, o valor da variável $I_{\mathrm{f}_{\mathrm{lim}}}$ relativa à máxima corrente permitida para o gerador 2 foi rebaixada para o valor de 2,2 [pu] em face do valor adotado para os geradores 1 e 3, de 2,5 [pu]. Deste modo, a barra 2 perde o controle de tensão da barra terminal, ilustrado na Figura 6.33, após a atuação do OXL, que ocorre em aproximadamente 165 segundos, conforme representado na Figura 6.34.

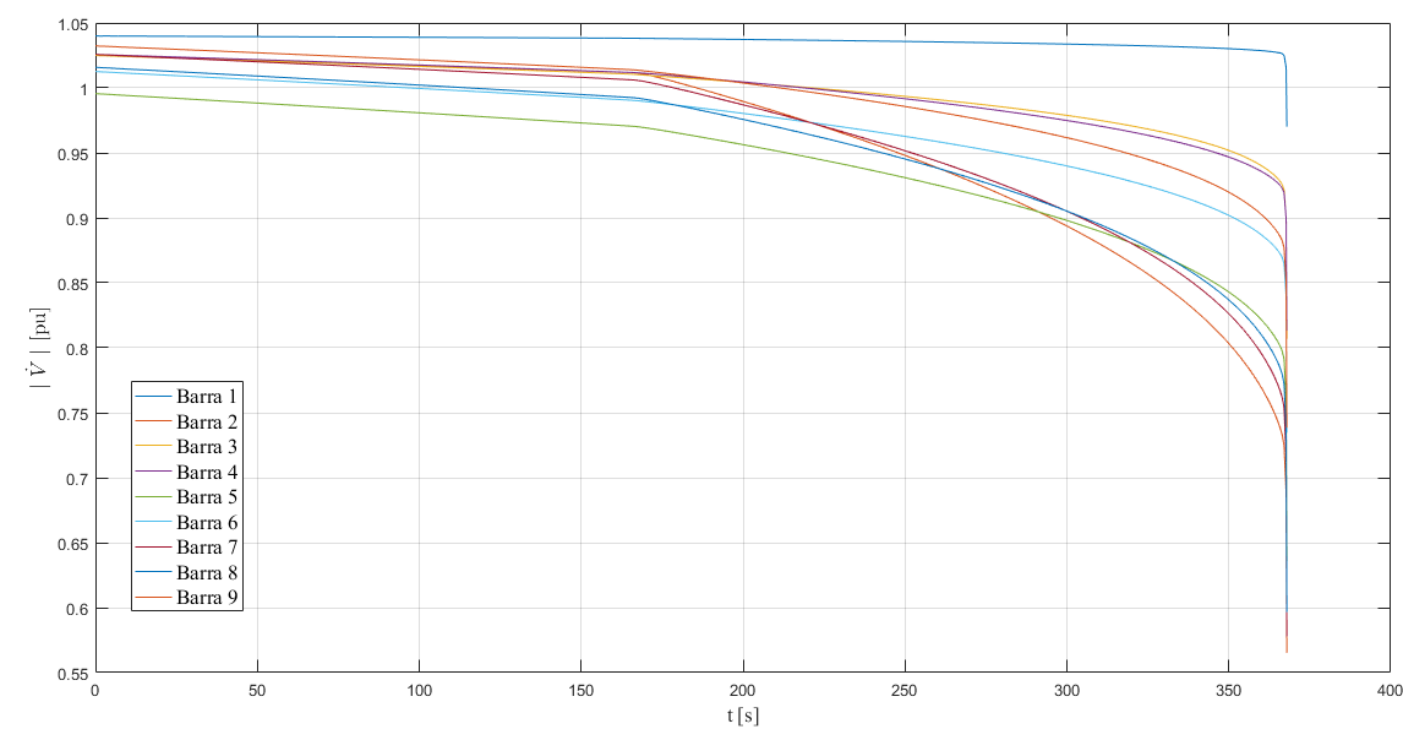

Figura 6.33: Gráfico da módulo da tensão em [pu] - caso 2 


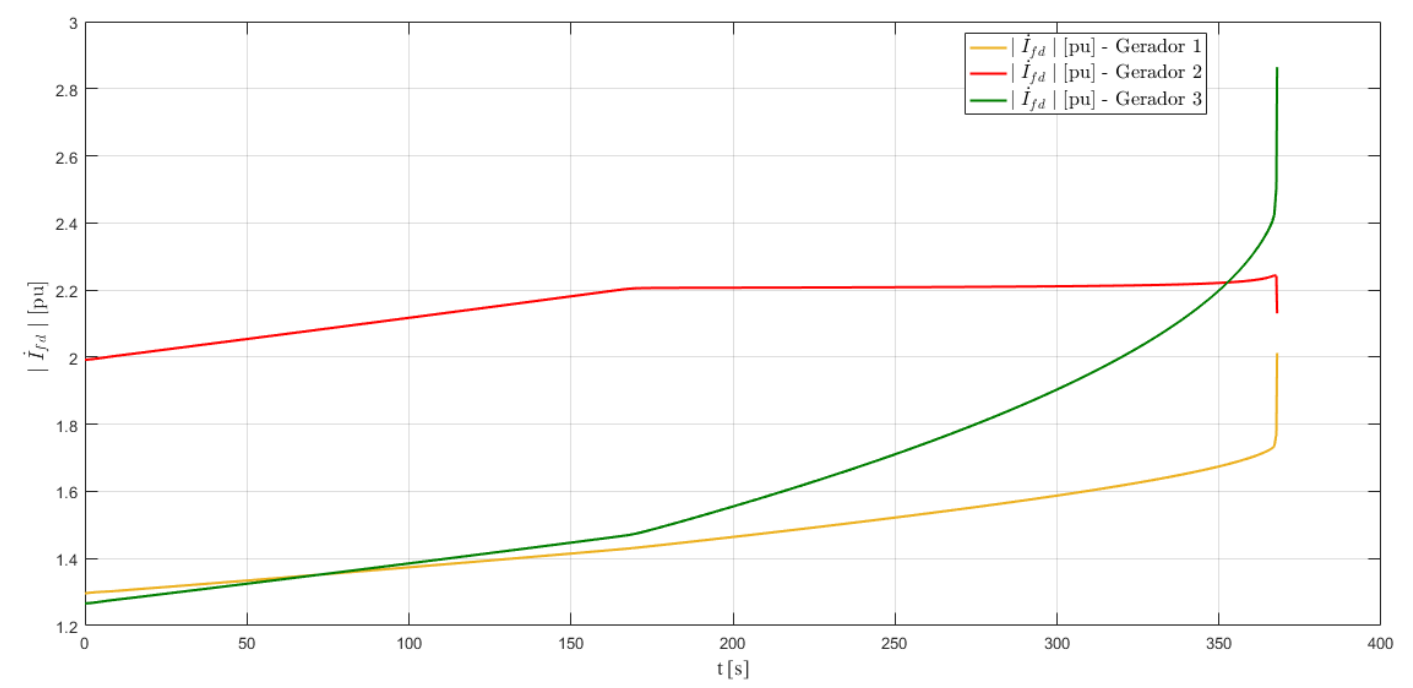

Figura 6.34: Corrente de campo em $[\mathrm{pu}]$ dos geradores - caso 2

O método de Corsi-Taranto foi ajustado neste caso com $k=0,005$ até a convergência e imediatamente após a atuação do OXL. Após a convergência do método, adotou-se $k=0,0001$.

Ambos os métodos respondem corretamente à atuação do OXL, interpretado no gráfico de impedância da Figura 6.35 pela variação abrupta de $\left|\bar{Z}_{t h}\right|$ estimado, e que se reflete na Figura 6.36 através da redução na margem de carregamento da barra 5, que atinge um novo valor em 5,2 segundos após a atuação do limitador de sobre-excitação, em 671, 4 segundos. A MTP é prevista pelos métodos ocorre em 367,7 segundos.

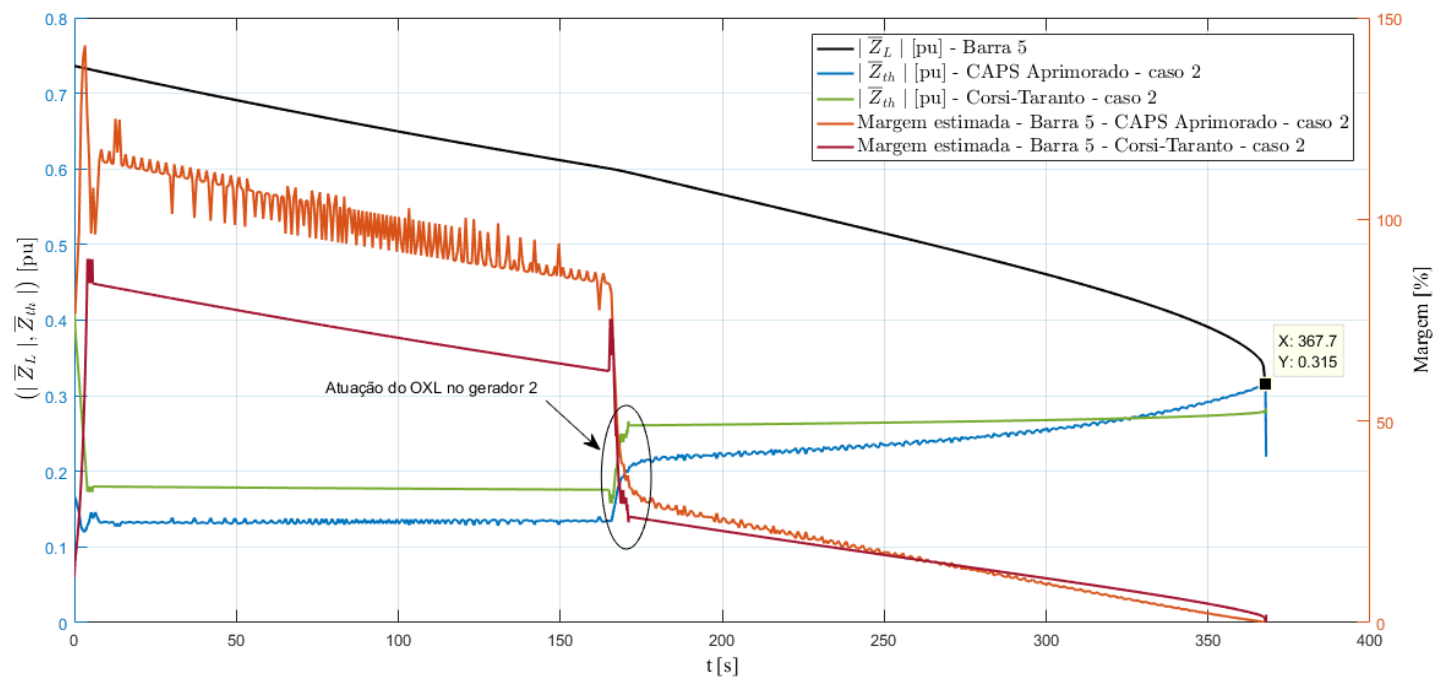

Figura 6.35: Gráfico de impedância em [pu] e margem de carregamento estimada em [\%] para a barra 5 - caso 2 
A margem da barra 2 apresenta um comportamento atípico na atuação do OXL, que corresponde à reconstrução das matrizes $\boldsymbol{Z}_{G G}$ e $\boldsymbol{K}_{G}$. Após este período, obverva-se que a margem cresce abruptamente, mas decresce de forma acentuada até se esgotar aos 160,9 segundos, portanto, com 6,8 segundos de antecedência.

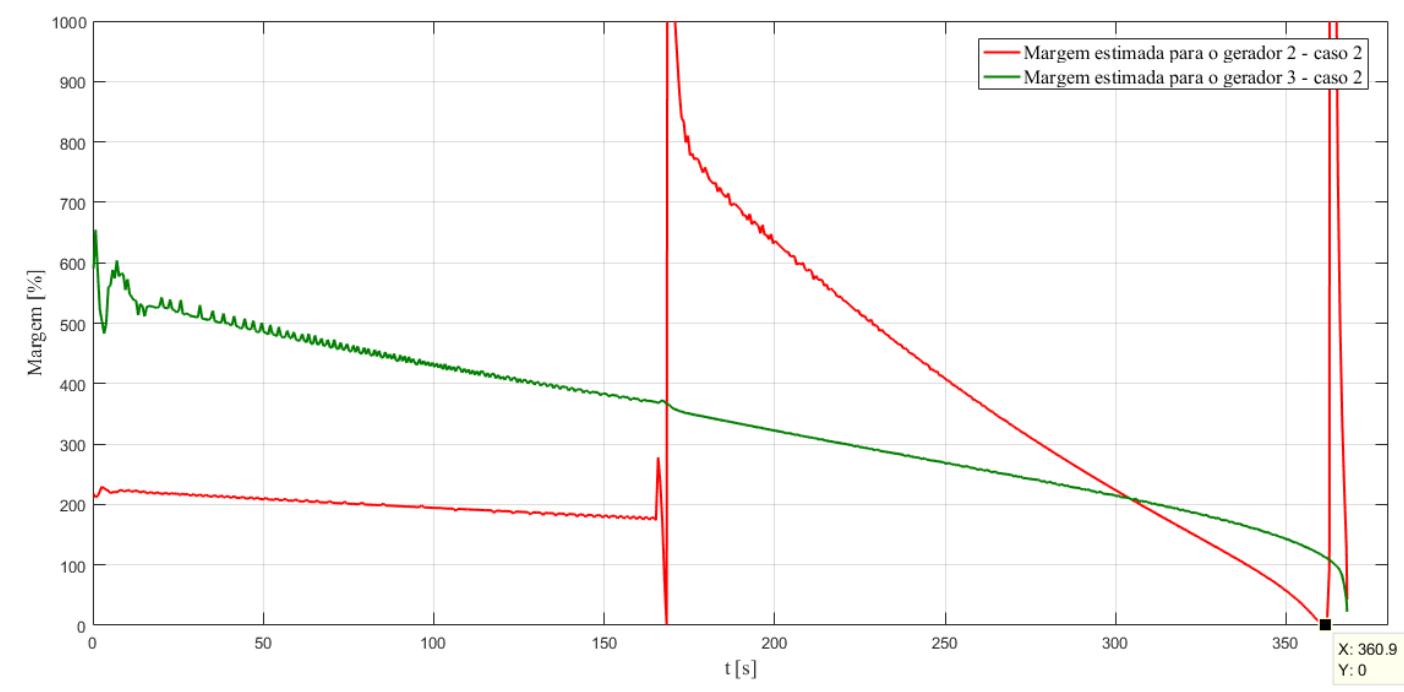

Figura 6.36: Margem estimada em [\%] para os geradores - Sistema WSCC 9 barras - caso 2 


\section{Conclusões}

A utilização de equivalentes de redes construídos a partir medições fasorias sincronizadas têm despertado, sobretudo nas últimas duas décadas, o interesse de pesquisadores que buscam sua aplicação para a avaliação das condições de estabilidade de tensão do sistema em tempo real. Sua relevância é tal, que continuamente são apresentados trabalhos baseados em novas metodologias ou aperfeiçoamento dos modelos já existentes.

Neste contexto, a dissertação representa uma contribuição ao tema através da investigação das características do método CAPS Aprimorado, e a partir de sua flexibilidade e da reconhecida necessidade de avaliação das barras de tensão controlada, adapta-lo a outros conjuntos de barras.

Os resultados inicialmente apresentados demostram que os equivalentes multi-portas, CAPS e CAPS Aprimorado, encontram aplicações como índices de avaliação das condições de estabilidade de tensão, identificando a barra de carga crítica em consonância com os métodos da matriz $\boldsymbol{D}^{\prime}$ e dos autovalores e autovetores. Destaca-se ainda que em relação ao método da matriz reduzida $\boldsymbol{J}_{R}^{Q}$, os modelos apresentam informações adicionais como a margem de carregamento estimada para a barra em análise.

Para validar sua precisão se estabelece um comparativo entre a margem prevista para os diversos sistemas teste àquela obtida pelo FPC. Com efeito, o modelo CAPS Aprimorado apresenta resultados com menor erro relativo, característica inclusive corroborada pelas análises apresentadas em [28].

Posteriormente comparou-se o desempenhos dos métodos VIP, ŠVG, Corsi-Taranto, CAPS e CAPS Aprimorado na identificação da MTP do sistema WSCC 9 barras. Esta simulação contempla distintas direções de carregamento através de sucessivos fluxos de carga até sua insolubilidade. Todos os modelos detectaram corretamente o ponto crítico, com exceção do método CAPS.

As limitações dos métodos foram apresentadas, demonstrando, por exemplo, que o método CAPS apresenta dificuldades quando é imposto ao sistema um crescimento de carga desproporcional, conforme descrito em [26]. Isto se deve à premissa do método com relação ao comportamento ligeiramente constante da impedância de acoplamento $\bar{Z}_{c j}$, apenas válida em crescimento de carga proporcional. Acrescenta-se ainda a "quebra" de acoplamento das car- 
gas em função de topologias específicas da rede, que produzem elementos nulos fora da diagonal principal da matriz $\boldsymbol{Z}_{L L}$.

Para o método VIP, destacaram-se dificuldades associadas ao mal condicionamento ou à singularidade da matriz dos coeficientes, e o método ŠVG reclama cuidados com relação à tolerância $|\Delta \dot{I}|^{\text {min }}$. Ambos os modelos, assim como o método CAPS, são sensível à presença de ruídos.

O algoritmo adaptativo de Corsi-Taranto, por sua vez, possui aplicações restritas a redes de EAT, e também requer uma escolha adequada do parâmetro de correção $k$ obtido empiricamente, embora a literatura seja mais consistente ao definir valores de referência [23, 24]. A aplicação da metododologia sob constante comprovação, inclusive no sistema elétrico italiano, reforçam sua superioridade em relação aos métodos baseados em medições locais, VIP e ŠVG.

Através das análises de sensibilidade às imprecisões adicionadas aos pseudofasores, mostrou-se que o método CAPS Aprimorado é fortemente influenciado, tendo seu desempenho deteriorado pela sensibilidade $\gamma$ que não responde adequadamente a estas condições.

As modificações sugeridas ao método CAPS Aprimorado são analisadas neste trabalho. Para as barras de transferência, propôs-se utilizar a soma dos fluxos que "deixam" a barra em análise como uma carga fictícia. Deste modo, o método CAPS Aprimorado deve processar a matriz de admitância nodal transformando-a em uma matriz de admitância nodal fictícia $\boldsymbol{Y}^{\text {fic }}$. A metodologia foi aplicada à barra 27 do sistema IEEE 30 barras e mostrou-se coerente ao identificar a MTP.

Com relação às barras de geração, a modificação sugerida emprega o parâmetro de sensibilidade $\psi$, tomando a variação de potência reativa com respeito a variação do módulo da tensão. Para a análise deste conjunto de barras, utilizou-se a simulação dinâmica com o sistema radial de 3 barras e o modelo WSCC 9 barras, implementados no PSAT.

Através da análise do sistema WSCC 9 barras, mostrou-se que o método CAPS Aprimorado apresenta resultados consistentes, antecipando em alguns segundos a iminente perda de estabilidade de tensão provocada pela máxima injeção de potência. Este requisito mostrou-se relevante, pois a identificação em tempo suficientemente anterior ao colapso de tensão permite a promoção das ações de controle restaurativo, como o corte de carga automático que pode ser realizado em até $150 \mathrm{~ms}$.

A simulação com o TG e OXL das máquinas desabilitados não foram apresentados nesta dissertação. Os resultados provenientes deste cenário, contudo, demonstrou que no instante em que se detecta a instabilidade de tensão, as barras de geração ainda apresentavam margem de potência para ser injetada 
na rede. Resultado semelhante ao obtido no sistema radial de 3 barras (casos 1 e 2), e demonstra que a responsabilidade sobre a MTP recai sobre o máximo fluxo de potência que chega à barra de carga.

O método CAPS Aprimorado encontra algumas dificuldades de implementação considerando este tipo de estudo, pois com a alta taxa de transferência de medições, o modelo produz oscilações indesejáveis. Para as simulações utilizadas no domínio do tempo diversos testes foram realizados para identificar o intervalo mínimo entre as medições, e aquele que demonstrou satisfatória convergência utilizou uma taxa de aquisição de 1 medição a cada 0,1 segundo.

Ainda assim, em alguns sistemas cuja simulação de eventos conduzem a grandes variações nos fasores de tensão e corrente, o método não apresenta um desempenho desejável. Este é o caso, por exemplo, do sistema Nordic apresentado em [31]. Neste sistema, um evento relativo à uma falta trifásica seguida da remoção da linha 4032 - 4044 produz resultados impróprios no modelo CAPS Aprimorado. Problemas semelhantes são relatados em [8] para o método CAPS Modificado, em que o autor optou pelo crescimento proporcional em uma barra específica do sistema, considerando as cargas do tipo corrente constante. Isto sugere que os modelos CAPS Aprimorado e CAPS Modificado podem apresentar limitações semelhantes pois empregam uma sensibilidade em sua modelagem.

\section{1}

\section{Sugestões para Trabalhos Futuros}

1. O método CAPS Modificado, brevemente comentado neste trabalho, apresenta um desempenho satisfatório na detecção da instabilidade de tensão. Embora o método possivelmente enfrente as mesmas limitações do modelo CAPS Aprimorado, uma confrontação entre ambos os métodos seria desejável para consolidar a superioridade do método CAPS Aprimorado, que exige uma infraestrutura de comunição mais simples devido à redução do número de medições requeridas e menor custo computacional.

2. Em [34] é desenvolvido um método para detecção da iminente perda da estabilidade de tensão provocada pela máxima injeção de potência, com resultados satisfatórios em sistemas de pequeno e grande porte. A metodologia antecipa a perda de estabilidade de tensão em alguns segundos empregando apenas medições locais e poucos recursos computacionais. Sendo assim, torna-se relevante uma análise comparativa baseada na resposta do modelo proposto na Seção 5.5.2 para avaliação das barras de 
geração e o método apresentado em [34], uma vez que tal estudo pode validar os resultados apresentados nesta dissertação.

3. Em [9] se estabelece uma análise comparativa entre os métodos de CorsiTaranto e Duong [35]. Considerando a modelagem dinâmica, o método de Duong necessita em sua formulação do valor associado à impedância do gerador. Com base nas simulações realizada em [9], percebe-se que os valores operacionais das máquinas não são adequados para o método, oferecendo, em alguns casos, um aumento no erro associado à previsão da MTP quando comparados aos valores negligenciados de $\bar{Z}_{\text {gerador }}$. Deste modo, o autor estabelece a impedâncica $\bar{Z}_{\text {fonte }}$ em contraste às impedâncias operacionais da máquina. Este valor é estimado pelo método de Corsi-Taranto através da forma indissociável $\bar{Z}_{\text {fonte }}=j X_{\text {fonte }}$, que o fornece como entrada ao método de Duong. Este trabalho apresenta um avanço neste sentido, e portanto, para complementar as avaliações relativas à estimação da impedância à montante da barra terminal, podese utilizar os valores obtidos pelo método CAPS Aprimorado, e aplica-los ao método de Duong. Espera-se que os valores estimados representem uma melhoria na detecção da MTP com relação aos valores apresentados em [9]. 


\section{Referências bibliográficas}

[1] EHRENSPERGER, J. G. "Sistemas de medição fasorial sincronizada : Análise do estado da arte e aplicações no monitoramento de sistemas de energia elétrica". Dissertação de mestrado, Universidade Federal de Santa Catarina, Florianópolis - SC, Brasil, 2004.

[2] PHADKE, A. G. "Synchronized phasor measurement in power systems". IEEE Computer Applications On Power Systems, 6:10-15, 1993.

[3] HART, D.G.; GHAROURE, V.; NOVOSEL, D.; et al. "PMUs - a new approach to power network monitoring". ABB Review 1/2001. pp. 58-61, 2001.

[4] ANDRADE, S. R. C. "Sistemas de medição fasorial sincronizada : Aplicações para melhoria da operação de sistemas elétricos de potência". Dissertação de mestrado, Universidade Federal de Minas Gerais, Belo Horizonte - MG, Brasil, 2008.

[5] YUAN, H.; LI, F. "A comparative study of measurement-based Thévenin equivalents identification methods". In: North American Power Symposium (NAPS), Pullman, WA, USA, pp. 1-6, Sep 2014.

[6] PALACIO, L. C. P. "Utilização de circuitos elétricos equivalentes na avaliação das condições de estabilidade de tensão". Dissertação de mestrado, Pontifícia Universidade Católica do Rio de Janeiro, Rio de Janeiro - RJ, Brasil, 2014.

[7] LAVENIUS, J.; VANFRETTI, L.; TARANTO, G. N. "Performance assessment of PMU-based estimation methods of Thevenin equivalents for real-time voltage stability monitoring". In: IEEE 15th International Conference on Environment and Electrical Engineering (EEEIC), Rome, Italy, pp. 10-13, Jun 2015.

[8] CARVALHO, C. S. "Comparação de métodos de identificação de instabilidade de tensão baseados em medições sincronizadas". Dissertação de mestrado, Universidade Federal do Rio de Janeiro, Rio de Janeiro - RJ, Brasil, 2016. 
[9] FERREIRA, A. P. F. "Comparação de dois métodos de detecção da instabilidade de tensão baseados em medição fasorial e equivalente de Thévenin". Dissertação de mestrado, Universidade Federal do Rio de Janeiro, Rio de Janeiro - RJ, Brasil, 2016.

[10] SILVA, F. D.; VALE, M. H. M.; DE SOUZA, C. E.; et al. "Equivalentes de redes para análise da estabilidade de tensão - Abordagem comparativa". In: The $12^{\text {th }}$ Latin-American Congress on Eletricity Generation and Transmission - CLAGTEE, Mar del Plata City, Argentina, Nov 2017.

[11] LIMA, D. C.; PRADA, R. B.; TEIXEIRA, M. V. P. "Análise comparativa de métodos baseados em medições fasorias para o monitoramento da estabilidade de tensão". In: XXII Congresso Brasileiro de Automática - CBA, João Pessoa - PB, Brasil, Sep 2018.

[12] KUNDUR, P.; PASERBA, J.; AJJARAPU, V.; et al. "Definition and classification of power system stability". IEEE Transaction on Power Systems. vol. 19, no. 3, pp. 1387-1401, Aug 2004.

[13] VAN CUTSEM, T.; MAILHOT, R. "Validation of a fast voltage stability analysis method on the Hydro-Québec System". IEEE Transaction on Power Systems, 1997. vol. 12, no. 1, pp. 282-292, Feb 1997.

[14] VEGA, J. L. L. "Avaliação e reforço das condições de estabilidade de tensão em barras de tensão controlada por geradores e compensadores síncronos". Tese de doutorado, Pontifícia Universidade Católica do Rio de Janeiro, Rio de Janeiro - RJ, Brasil, 2009.

[15] PRADA R. B.; PALOMINO E. G. C.; DOS SANTOS, J. O. R.; et al. "Voltage stability assessment for real-time operation". IEE Proceedings - Generation, Transmission \& Distribution, March 2002. vol. 149, no. 2, pp. 282-292, Mar 2002.

[16] GAO, B.; MORISON, G. K.; KUNDUR, P. "Voltage stability evaluation using modal analysis". IEEE Transactions on Power Systems. vol. 7, no. 4, pp. 1529-1542, Nov 1992.

[17] PINZÓN, J. D.; COLOMÉ, D. G. "Voltage stability assessment using synchrophasor measurements: Trends and development". In: Proc. IEEE PES Innovative Smart Grid Technologies Conference - Latin America, Quito, Ecuador, pp. 20-22, Sep 2017. 
[18] GLAVIC, M.; VAN CUTSEM, T. "A short survey of methods for voltage instability detection". In: Proc. IEEE Power and Energy Society General Meeting, San Diego, CA, USA, pp. 24-29, Jul 2011.

[19] VU K.; BEGOVIC M. M.; NOVOSEL D.; et al. "Use of local measurements to estimate voltage-stability margin". IEEE Transaction on Power Systems. vol. 14, no. 3, pp. 1029-1035, Aug 1999.

[20] JULIAN, D. E.; SCHULZ, R. P.; VU, K. T.; et al. "Quantifying proximity to voltage collapse using the Voltage Instability Predictor (VIP)". In: Power Engineering Society Summer Meeting, Seattle, WA, USA, pp. 16-20, Jul 2000.

[21] ŠMON, I.; VERBIČ G.; GUBINA F. "Local voltage-stability index using Tellegen's theorem". IEEE Transactions on Power Systems. vol. 21, no. 3, pp. 1267-1275, Aug 2006.

[22] PENFIELD, P.; SPENCE, R.; DUINKER, S. "A generalized form of Tellegen's theorem". IEEE Transactions on Power Systems. vol. 17, no. 3, pp. 302-305, Aug 1970.

[23] CORSI, S.; TARANTO, G. N. "A real-time voltage instability identification algorithm based on local phasor measurements". IEEE Transactions on Power Systems. vol. 23, no. 3, pp. 1271-1279, Aug 2008.

[24] INFANTE, C. A. O. "Detecção da proximidade de instabilidade de tensão utilizando sincrofasores". Dissertação de mestrado, Universidade Federal do Rio de Janeiro, Rio de Janeiro - RJ, Brasil, 2012.

[25] WANG, Y.; RAHIMI, I.; LI, W.; et al. "Voltage stability monitoring based on the concept of coupled single-port circuit". IEEE Transactions on Power Systems. vol. 26, no. 4, pp. 2154-2163, Nov 2011.

[26] LIU, J. -H.; CHU, C. -C. "PMU measurement-based voltage stability indicators by modified coupled single-port models". IEEE Transaction on Power Systems. vol. 29, no. 2, pp. 756-764, Mar 2014.

[27] YUAN, H.; LI, X.; LI, F.; et al. "Mitigate overestimation of voltage stability margin by coupled single-port circuit models". In: Power and Energy Society General Meeting (PESGM), Boston, MA, USA, pp. 17-21, Jul 2016.

[28] CUI, B.; WANG, Z.. "Voltage stability assessment based on improved coupled single-port method". IET Generation, Transmission \& Distribution. vol. 11, no. 10, pp. 2703-2711, Jun 2017. 
[29] STAGG, G. W.; EL-ABIAD, A. H.. "Computer methods in power system analysis". McGraw-Hill Inc., 1968.

[30] AJJARAPU, V.. "Computational techniques for voltage stability assessment and control'. Springer, 2006.

[31] VAN CUTSEM, V.; GLAVIC, M.; ROSEHART, W.; et al. "Test systems for voltage stability analysis and security assessment". Technical report, Institute of Electrical and Electronics Engineers (IEEE). Aug 2015. Report No.: PES-TR 19.

[32] KUNDUR, P.. "Power system stability and control". McGraw-Hill, New York, 1994.

[33] MILANO, F. "Power system analysis toolbox". Technical report. Documentation for PSAT version 1.3.4, Jul 2005.

[34] TEIXEIRA, M. V. P. "Análises estática e dinâmica da atuação de equipamentos de controle na estabilidade de tensão". Tese de doutorado, Pontifícia Universidade Católica do Rio de Janeiro, Rio de Janeiro - RJ, Brasil, 2016.

[35] DUONG, D. T; UHLEN, K.; LØVLUND, S. "A method for real time voltage stability monitoring in sub-transmission networks". In: Power Systems Computation Conference, Wroclaw, Poland, Feb 2014. 
A

Cálculo da Sensibilidade Utilizada no Método CAPS Aprimorado

Ao tomar a barra $j$ em análise a partir do método CAPS Aprimorado, obtém-se o sistema equivalente duas barras mostrado na Figura A.1, de onde segue a relação:

$$
\dot{E}_{e q_{j}}^{\prime}=\dot{V}_{j}+\dot{V}_{\text {linha }}
$$

O diagrama fasorial correspodente a equação (A.1) é apresentado na Figura A.2.

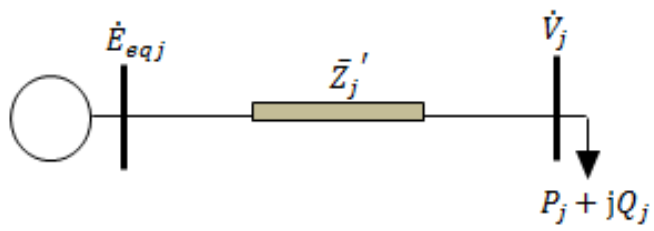

Figura A.1: Equivalente "visto" pela barra $j$ em análise

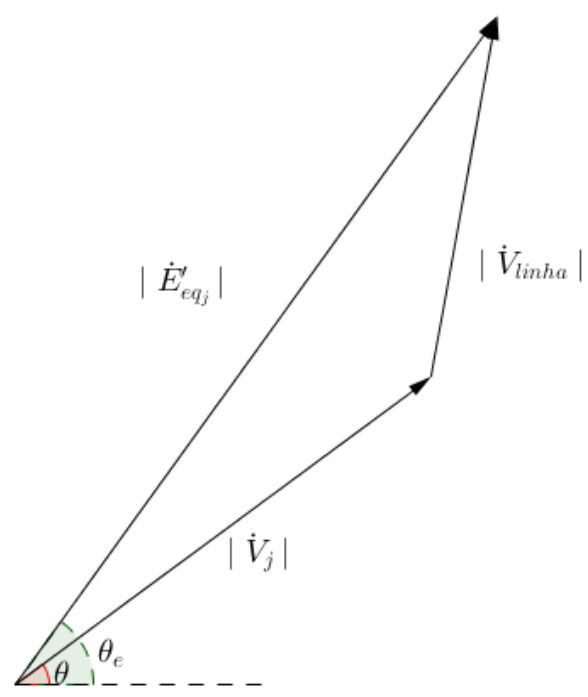

Figura A.2: Diagrama fasorial

Deste modo, é possível obter o módulo correspondente a queda tensão na linha aplicando a lei dos cossenos, pois:

$$
\left|\dot{V}_{\text {linha }}\right|^{2}=\left|\dot{E}_{e q_{j}}^{\prime}\right|^{2}+\left|\dot{V}_{j}\right|^{2}-2\left|\dot{E}_{e q_{j}}^{\prime}\right|\left|\dot{V}_{j}\right| \cos \left(\theta_{e}-\theta\right)
$$


Apêndice A. Cálculo da Sensibilidade Utilizada no Método CAPS Aprimorađđđ8

A identidade (A.2) será considerada nas demonstrações que se seguem.

Partindo da equação associada ao fluxo de potência no sistema equivalente, e nomeando-a $\Phi\left(\left|\dot{V}_{j}\right|, P_{j}\right)$, tem-se que:

$\Phi\left(\left|\dot{V}_{j}\right|, P_{j}\right)=\left|\dot{V}_{j}\right|^{4}-\left[\left|\dot{E}_{e q_{j}}^{\prime}\right|^{2}-2\left(R_{j}^{\prime} P_{j}+X_{j}^{\prime} Q_{j}\right)\right]\left|\dot{V}_{j}\right|^{2}+\left(P_{j}^{2}+Q_{j}^{2}\right)\left(R_{j}^{\prime 2}+X_{j}^{\prime 2}\right)=0$

logo:

$$
\frac{\partial \Phi\left(\left|\dot{V}_{j}\right|, P_{j}\right)}{\partial P_{j}}=2\left|\dot{V}_{j}\right|^{2}\left(R_{j}^{\prime}+X_{j}^{\prime} \frac{Q_{j}}{P_{j}}\right)+\left(R_{j}^{\prime 2}+X_{j}^{\prime 2}\right)\left(2 P_{j}+2 \frac{Q_{j} P_{j}}{P_{j}^{2}}\right)
$$

reorganizando a expressão:

$$
\frac{\partial \Phi\left(\left|\dot{V}_{j}\right|, P_{j}\right)}{\partial P_{j}}=\frac{2\left|\dot{V}_{j}\right|^{2}}{P_{j}}\left(R_{j} P_{j}+X_{j} Q_{j}\right)+\left(R_{j}^{\prime 2}+X_{j}^{\prime 2}\right)\left(\frac{2 P_{j}^{2}+2 Q_{j}^{2}}{P_{j}}\right)
$$

sendo $\left|\bar{Z}_{j}^{\prime}\right|^{2}=R_{j}^{\prime 2}+X_{j}^{\prime 2}$ e $\left|\dot{S}_{j}\right|^{2}=P_{j}^{2}+Q_{j}^{2}$, obtém-se:

$$
\begin{gathered}
\frac{\partial \Phi\left(\left|\dot{V}_{j}\right|, P_{j}\right)}{\partial P_{j}}=\frac{2\left|\dot{V}_{j}\right|^{2}}{P_{j}}\left(R_{j}^{\prime} P_{j}+X_{j}^{\prime} Q_{j}\right)+\frac{2\left|\bar{Z}_{j}^{\prime}\right|^{2}\left|\dot{S}_{j}\right|^{2}}{P_{j}} \therefore \\
\frac{\partial \Phi\left(\left|\dot{V}_{j}\right|, P_{j}\right)}{\partial P_{j}}=\frac{2\left|\dot{V}_{j}\right|^{2}}{P_{j}}\left(R_{j}^{\prime} P_{j}+X_{j}^{\prime} Q_{j}\right)+\frac{2\left|\dot{V}_{\text {linha }}\right|^{2}\left|\dot{V}_{j}\right|^{2}\left|\dot{I}_{j}\right|^{2}}{P_{j}\left|\dot{I}_{j}\right|^{2}} \therefore \\
\frac{\partial \Phi\left(\left|\dot{V}_{j}\right|, P_{j}\right)}{\partial P_{j}}=\frac{2\left|\dot{V}_{j}\right|^{2}}{P_{j}}\left(R_{j}^{\prime} P_{j}+X_{j}^{\prime} Q_{j}+\left|\dot{V}_{\text {linha }}\right|^{2}\right)
\end{gathered}
$$

utilizando a identidade $R_{j}^{\prime} P_{j}+X_{j}^{\prime} Q_{j}=\Re\left\{\bar{Z}_{j}^{\prime} \dot{S}_{j}^{*}\right\}$ :

$$
\begin{gathered}
\frac{\partial \Phi\left(\left|\dot{V}_{j}\right|, P_{j}\right)}{\partial P_{j}}=\frac{2\left|\dot{V}_{j}\right|^{2}}{P_{j}}\left(\Re\left\{\bar{Z}_{j}^{\prime} \dot{S}_{j}^{*}\right\}+\left|\dot{V}_{\text {linha }}\right|^{2}\right) \therefore \\
\frac{\partial \Phi\left(\left|\dot{V}_{j}\right|, P_{j}\right)}{\partial P_{j}}=\frac{2\left|\dot{V}_{j}\right|^{2}}{P_{j}}\left(\Re\left\{\left(\frac{\dot{E}_{e q_{j}}^{\prime}-\dot{V}_{j}}{\dot{I}_{j}}\right) \dot{V}_{j}^{*} \dot{I}_{j}\right\}+\left|\dot{V}_{\text {linha }}\right|^{2}\right) \therefore \\
\frac{\partial \Phi\left(\left|\dot{V}_{j}\right|, P_{j}\right)}{\partial P_{j}}=\frac{2\left|\dot{V}_{j}\right|^{2}}{P_{j}}\left(\Re\left\{\dot{E}_{e q_{j}}^{\prime} \dot{V}_{j}-\left|\dot{V}_{j}\right|^{2}\right\}+\left|\dot{V}_{\text {linha }}\right|^{2}\right) \therefore
\end{gathered}
$$


Apêndice A. Cálculo da Sensibilidade Utilizada no Método CAPS Aprimorađdđ9

$$
\begin{gathered}
\frac{\partial \Phi\left(\left|\dot{V}_{j}\right|, P_{j}\right)}{\partial P_{j}}=\frac{2\left|\dot{V}_{j}\right|^{2}}{P_{j}}\left(\left|\dot{E}_{e q_{j}}^{\prime}\right|\left|\dot{V}_{j}\right| \cos \left(\theta_{e}-\theta\right)-\left|\dot{V}_{j}\right|^{2}+\left|\dot{V}_{\text {linha }}\right|^{2}\right) \therefore \\
\frac{\partial \Phi\left(\left|\dot{V}_{j}\right|, P_{j}\right)}{\partial P_{j}}=\frac{2\left|\dot{V}_{j}\right|^{2}\left|\dot{E}_{e q_{j}}^{\prime}\right|}{P_{j}}\left(\left|\dot{E}_{e q_{j}}^{\prime}\right|-\left|\dot{V}_{j}\right| \cos \left(\theta_{e}-\theta\right)\right)
\end{gathered}
$$

Por sua vez, tomando a derivada parcial de $\Phi$ com respeito a $\left|\dot{V}_{j}\right|$ :

$$
\frac{\partial \Phi\left(\left|\dot{V}_{j}\right|, P_{j}\right)}{\partial\left|\dot{V}_{j}\right|}=4\left|\dot{V}_{j}\right|^{3}-2\left|\dot{V}_{j}\right|\left[\left|\dot{E}_{e q_{j}}^{\prime}\right|^{2}-2\left(R_{j} P_{j}+X_{j} Q_{j}\right)\right]
$$

considerando a já conhecida identidade $R_{j}^{\prime} P_{j}+X_{j}^{\prime} Q_{j}=\Re\left\{\bar{Z}_{j}^{\prime} \dot{S}_{j}^{*}\right\}$ :

$$
\frac{\partial \Phi\left(\left|\dot{V}_{j}\right|, P_{j}\right)}{\partial\left|\dot{V}_{j}\right|}=4\left|\dot{V}_{j}\right|^{3}-2\left|\dot{V}_{j}\right|\left(\left|\dot{V}_{j}\right|^{2}+\left|\dot{V}_{\text {linha }}\right|^{2}\right) \therefore
$$

$$
\frac{\partial \Phi\left(\left|\dot{V}_{j}\right|, P_{j}\right)}{\partial\left|\dot{V}_{j}\right|}=4\left|\dot{V}_{j}\right|^{3}-2\left|\dot{V}_{j}\right|\left(2\left|\dot{V}_{j}\right|^{2}+\left|\dot{E}_{e q_{j}}^{\prime}\right|^{2}-2\left|\dot{E}_{e q_{j}}^{\prime}\right|\left|\dot{V}_{j}\right| \cos \left(\theta_{e}-\theta\right)\right)
$$

Portanto:

$$
\frac{\partial \Phi\left(\left|\dot{V}_{j}\right|, P_{j}\right)}{\partial\left|\dot{V}_{j}\right|}=-2\left|\dot{V}_{j}\right|\left|\dot{E}_{e q_{j}}^{\prime}\right|\left(\left|\dot{E}_{e q_{j}}^{\prime}\right|-2\left|\dot{V}_{j}\right| \cos \left(\theta_{e}-\theta\right)\right)
$$




\section{B \\ Dados de Linha e Geração}

As tabelas abaixo referem-se aos dados de geração e de linha utilizados neste trabalho para a avaliação estática da estabilidade de tensão.

\section{B.1}

\section{Sistema IEEE 14 Barras}

Tabela B.1: Dados de geração do sistema IEEE 14 barras

\begin{tabular}{|ccc|}
\hline Barra & $Q_{G}^{\text {mín }}[\mathrm{pu}]$ & $Q_{G}^{\text {máx }}[\mathrm{pu}]$ \\
\hline \hline 1 & $-9,99$ & 9,99 \\
2 & $-0,40$ & 0,50 \\
3 & 0,00 & 0,40 \\
6 & $-0,06$ & 0,24 \\
8 & -0.06 & 0,24 \\
\hline
\end{tabular}

Tabela B.2: Dados de linha do sistema IEEE 14 barras

\begin{tabular}{|cccccc|}
\hline$\# k$ & $\# m$ & $r_{k m}[\mathrm{pu}]$ & $x_{k m}[\mathrm{pu}]$ & $b_{k m}^{s h} / 2[\mathrm{pu}]$ & $m[\mathrm{pu} / \mathrm{pu}]$ \\
\hline 1 & 2 & 0,01938 & 0,05917 & 0,0264 & 1,000 \\
1 & 5 & 0,05403 & 0,22304 & 0,0246 & 1,000 \\
2 & 3 & 0,04699 & 0,19797 & 0,0219 & 1,000 \\
2 & 4 & 0,05811 & 0,17632 & 0,0187 & 1,000 \\
2 & 5 & 0,05695 & 0,17388 & 0,0170 & 1,000 \\
3 & 4 & 0,06701 & 0,17103 & 0,0173 & 1,000 \\
4 & 5 & 0,01335 & 0,04211 & 0,0064 & 1,000 \\
4 & 7 & 0,00000 & 0,20912 & 0,0000 & 0,978 \\
4 & 9 & 0,00000 & 0,55618 & 0,0000 & 0,968 \\
5 & 6 & 0,00000 & 0,25202 & 0,0000 & 0,932 \\
6 & 11 & 0,09498 & 0,19890 & 0,0000 & 1,000 \\
6 & 12 & 0,12291 & 0,25581 & 0,0000 & 1,000 \\
6 & 13 & 0,06615 & 0,13027 & 0,0000 & 1,000 \\
7 & 8 & 0,00000 & 0,17615 & 0,0000 & 1,000 \\
\hline
\end{tabular}

Continuação na próxima página 
Tabela B.2 - Continuação da página anterior

\begin{tabular}{|cccccc|}
\hline$\# k$ & $\# m$ & $r_{k m}[\mathrm{pu}]$ & $x_{k m}[\mathrm{pu}]$ & $b_{k m}^{s h} / 2[\mathrm{pu}]$ & $m[\mathrm{pu} / \mathrm{pu}]$ \\
\hline 7 & 9 & 0,00000 & 0,11001 & 0,0000 & 1,000 \\
9 & 10 & 0,03181 & 0,08450 & 0,0000 & 1,000 \\
9 & 14 & 0,12711 & 0,27038 & 0,0000 & 1,000 \\
10 & 11 & 0,08205 & 0,19207 & 0,0000 & 1,000 \\
12 & 13 & 0,22092 & 0,19988 & 0,0000 & 1,000 \\
13 & 14 & 0,17093 & 0,34802 & 0,0000 & 1,000 \\
\hline
\end{tabular}

\section{B.2}

\section{Sistema WSCC 9 Barras}

Tabela B.3: Dados de geração do sistema WSCC 9 barras

\begin{tabular}{|ccc|}
\hline Barra & $Q_{G}^{\text {mín }}[\mathrm{pu}]$ & $Q_{G}^{\text {máx }}[\mathrm{pu}]$ \\
\hline \hline 1 & $-9,99$ & 9,99 \\
2 & $-9,99$ & 9,99 \\
3 & -9.99 & 9,99 \\
\hline
\end{tabular}

Tabela B.4: Dados de linha do sistema WSCC 9 barras

\begin{tabular}{|cccccc|}
\hline$\# k$ & $\# m$ & $r_{k m}[\mathrm{pu}]$ & $x_{k m}[\mathrm{pu}]$ & $b_{k m}^{s h} / 2[\mathrm{pu}]$ & $m[\mathrm{pu} / \mathrm{pu}]$ \\
\hline 1 & 4 & 0,0000 & 0,0576 & 0,0000 & 1,000 \\
2 & 7 & 0,0000 & 0,0625 & 0,0000 & 1,000 \\
3 & 9 & 0,0000 & 0,0586 & 0,0000 & 1,000 \\
4 & 5 & 0,0100 & 0,0850 & 0,0880 & 1,000 \\
4 & 6 & 0,0170 & 0,0920 & 0,0790 & 1,000 \\
5 & 7 & 0,0320 & 0,1610 & 0,1530 & 1,000 \\
6 & 9 & 0,0390 & 0,1700 & 0,1790 & 1,000 \\
7 & 8 & 0,0085 & 0,0720 & 0,0745 & 1,000 \\
8 & 9 & 0,0119 & 0,1008 & 0,1045 & 1,000 \\
\hline
\end{tabular}




\section{B.3}

\section{Sistema IEEE 30 Barras}

Tabela B.5: Dados de geração do sistema IEEE 30 barras

\begin{tabular}{|ccc|}
\hline Barra & $Q_{G}^{\text {mín }}[\mathrm{pu}]$ & $Q_{G}^{\text {máx }}[\mathrm{pu}]$ \\
\hline \hline 1 & $-9,99$ & 9,99 \\
2 & $-0,40$ & 0,50 \\
5 & $-0,40$ & 0,40 \\
8 & $-0,10$ & 0,40 \\
11 & $-0,06$ & 0,24 \\
13 & -0.06 & 0,24 \\
\hline
\end{tabular}

Tabela B.6: Dados de linha do sistema IEEE 30 barras

\begin{tabular}{|cccccc|}
\hline$\# k$ & $\# m$ & $r_{k m}[\mathrm{pu}]$ & $x_{k m}[\mathrm{pu}]$ & $b_{k m}^{s h} / 2[\mathrm{pu}]$ & $m[\mathrm{pu} / \mathrm{pu}]$ \\
\hline 1 & 2 & 0,0192 & 0,0575 & 0,0264 & 1,000 \\
1 & 3 & 0,0452 & 0,1652 & 0,0204 & 1,000 \\
2 & 4 & 0,0570 & 0,1737 & 0,0184 & 1,000 \\
3 & 4 & 0,0132 & 0,0379 & 0,0042 & 1,000 \\
2 & 5 & 0,0472 & 0,1983 & 0,0209 & 1,000 \\
2 & 6 & 0,0581 & 0,1763 & 0,0187 & 1,000 \\
4 & 6 & 0,0119 & 0,0414 & 0,0045 & 1,000 \\
5 & 7 & 0,0460 & 0,1160 & 0,0102 & 1,000 \\
6 & 7 & 0,0267 & 0,0820 & 0,0085 & 1,000 \\
6 & 8 & 0,0120 & 0,0420 & 0,0045 & 1,000 \\
6 & 9 & 0,0000 & 0,2080 & 0,0000 & 0,978 \\
6 & 10 & 0,0000 & 0,5560 & 0,0000 & 0,969 \\
7 & 11 & 0,0000 & 0,2080 & 0,0000 & 1,000 \\
7 & 10 & 0,0000 & 0,1100 & 0,0000 & 1,000 \\
4 & 12 & 0,0000 & 0,2560 & 0,0000 & 0,932 \\
12 & 13 & 0,0000 & 0,1400 & 0,0000 & 1,000 \\
12 & 14 & 0,1231 & 0,2559 & 0,0000 & 1,000 \\
12 & 15 & 0,0662 & 0,1304 & 0,0000 & 1,000 \\
12 & 16 & 0,0945 & 0,1987 & 0,0000 & 1,000 \\
14 & 15 & 0,2210 & 0,1997 & 0,0000 & 1,000 \\
16 & 17 & 0,0524 & 0,1923 & 0,0000 & 1,000 \\
15 & 18 & 0,1073 & 0,2185 & 0,0000 & 1,000 \\
18 & 19 & 0,0639 & 0,1292 & 0,0000 & 1,000 \\
\hline
\end{tabular}

Continuação na próxima página 
Tabela B.6 - Continuação da página anterior

\begin{tabular}{|cccccc|}
\hline$\# k$ & $\# m$ & $r_{k m}[\mathrm{pu}]$ & $x_{k m}[\mathrm{pu}]$ & $b_{k m}^{s h} / 2[\mathrm{pu}]$ & $m[\mathrm{pu} / \mathrm{pu}]$ \\
\hline 19 & 20 & 0,0340 & 0,0680 & 0,0000 & 1,000 \\
10 & 20 & 0,0936 & 0,2090 & 0,0000 & 1,000 \\
10 & 17 & 0,0324 & 0,0845 & 0,0000 & 1,000 \\
10 & 21 & 0,0348 & 0,0749 & 0,0000 & 1,000 \\
10 & 22 & 0,0727 & 0,1499 & 0,0000 & 1,000 \\
21 & 22 & 0,0116 & 0,0236 & 0,0000 & 1,000 \\
15 & 23 & 0,1000 & 0,2020 & 0,0000 & 1,000 \\
22 & 24 & 0,1150 & 0,1790 & 0,0000 & 1,000 \\
23 & 24 & 0,1320 & 0,2700 & 0,0000 & 1,000 \\
24 & 25 & 0,1885 & 0,3292 & 0,0000 & 1,000 \\
25 & 26 & 0,2544 & 0,3800 & 0,0000 & 1,000 \\
25 & 27 & 0,1093 & 0,2087 & 0,0000 & 1,000 \\
28 & 27 & 0,0000 & 0,3960 & 0,0000 & 0,968 \\
27 & 29 & 0,2198 & 0,4153 & 0,0000 & 1,000 \\
27 & 30 & 0,3202 & 0,6027 & 0,0000 & 1,000 \\
29 & 30 & 0,2399 & 0,4533 & 0,0000 & 1,000 \\
8 & 28 & 0,0636 & 0,2000 & 0,0214 & 1,000 \\
6 & 28 & 0,0169 & 0,0599 & 0,0065 & 1,000 \\
\hline
\end{tabular}




\section{C}

\section{Dados dos Controles - Sistema WSCC 9 Barras}

As tabelas e figuras abaixo referem-se aos controles utilizados no sistema WSCC 9 barras apresentado na Seção 6.5.2. O PSAT oferece a possibilidade de representação de dois modelos distintos para o TG e três modelos para o AVR. Para este trabalho adotou-se o TG do Tipo II mostrado na Figura C.1, o AVR do Tipo II encontrado na Figura C.2 e o limitador de sobre-excitação representado na Figura C.3. Os parâmetros adotados para os modelos de TG, AVR e OXL encontram-se, respectivamente, nas Tabelas C.1, C.2, C.3 e C.4.

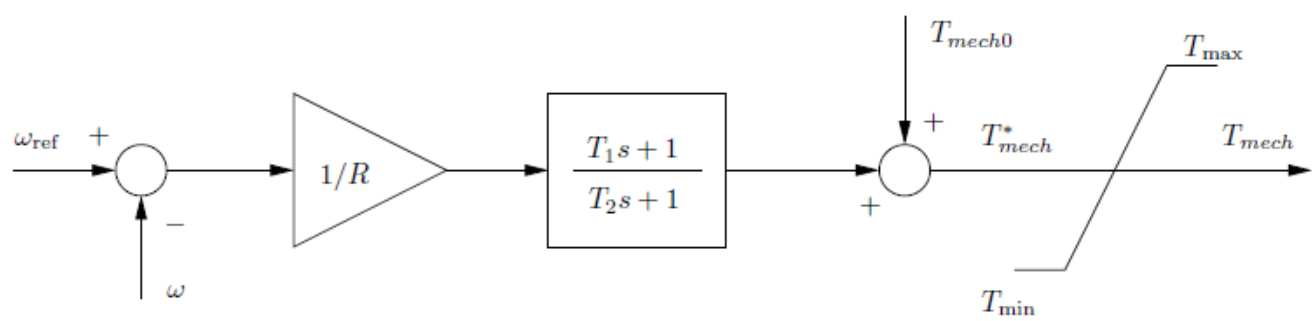

Figura C.1: Regulador de velocidade do tipo II

Tabela C.1: Dados do regulador de velocidade

\begin{tabular}{|ccc|}
\hline Variável & Valor & Unidade \\
\hline \hline$\omega_{\text {ref }}$ & 1 & $\mathrm{pu}$ \\
$R$ & 0,02 & $\mathrm{pu}$ \\
$T_{\text {máx }}$ & 2,2 & $\mathrm{pu}$ \\
$T_{\text {mín }}$ & 0 & $\mathrm{pu}$ \\
$T_{2}$ & 10 & $\mathrm{~s}$ \\
$T_{1}$ & 1 & $\mathrm{~s}$ \\
\hline
\end{tabular}




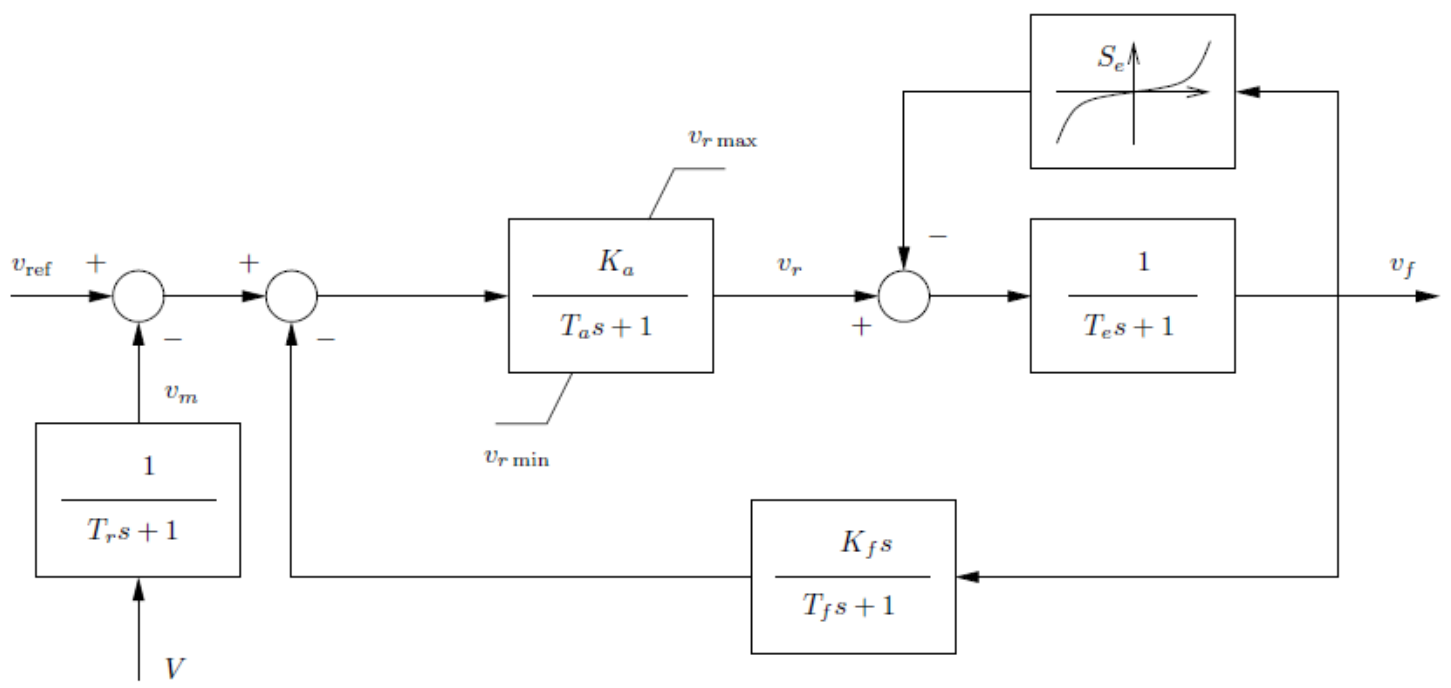

Figura C.2: Regulador automático de tensão do tipo II

Tabela C.2: Dados do regulador automático de tensão

\begin{tabular}{|ccc|}
\hline Variável & Valor & Unidade \\
\hline \hline$V_{r \max }$ & 5,00 & $\mathrm{pu}$ \\
$V_{r \min }$ & $-5,00$ & $\mathrm{pu}$ \\
$K_{a}$ & 20 & $\mathrm{pu} / \mathrm{pu}$ \\
$T_{a}$ & 0,2 & $\mathrm{~s}$ \\
$K_{f}$ & 0,063 & $\mathrm{pu} / \mathrm{pu}$ \\
$T_{f}$ & 0,35 & $\mathrm{~s}$ \\
$K_{e}$ & 1 & $\mathrm{pu} / \mathrm{pu}$ \\
$T_{e}$ & 0,314 & $\mathrm{~s}$ \\
$T_{r}$ & 0,001 & $\mathrm{~s}$ \\
$A$ & 0,0039 & \\
$B$ & 1,555 & \\
\hline
\end{tabular}




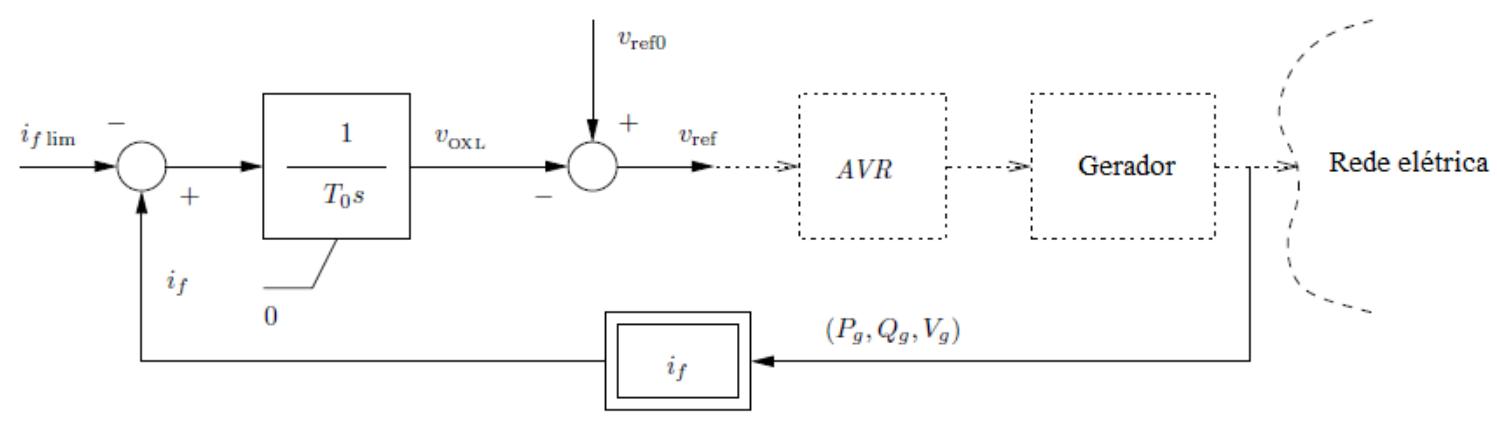

Figura C.3: Limitador de sobre-excitação

Tabela C.3: Dados do limitador de sobre-excitação dos geradores 1 e 3

\begin{tabular}{|ccc|}
\hline Variável & Valor & Unidade \\
\hline \hline$T_{0}$ & 10 & $\mathrm{~s}$ \\
$I_{f l i m}$ & 2,5 & $\mathrm{pu}$ \\
$v_{\text {OXL }}$ & 0,5 & $\mathrm{pu}$ \\
\hline
\end{tabular}

Tabela C.4: Dados do limitador de sobre-excitação do gerador 2

\begin{tabular}{|ccc|}
\hline Variável & Valor & Unidade \\
\hline \hline$T_{0}$ & 10 & $\mathrm{~s}$ \\
$I_{f l i m}$ & 2,2 & $\mathrm{pu}$ \\
$v_{\text {OXL }}$ & 0,5 & $\mathrm{pu}$ \\
\hline
\end{tabular}

